\title{
ECAR 4951 BSU-8242 3 DPA As-Run Physics Analysis
}

\section{July 2020}

Jason V Brookman

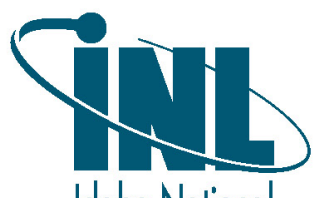

Idaho National

Laboratory 


\section{DISCLAIMER}

This information was prepared as an account of work sponsored by an agency of the U.S. Government. Neither the U.S. Government nor any agency thereof, nor any of their employees, makes any warranty, expressed or implied, or assumes any legal liability or responsibility for the accuracy, completeness, or usefulness, of any information, apparatus, product, or process disclosed, or represents that its use would not infringe privately owned rights. References herein to any specific commercial product, process, or service by trade name, trade mark, manufacturer, or otherwise, does not necessarily constitute or imply its endorsement, recommendation, or favoring by the U.S. Government or any agency thereof. The views and opinions of authors expressed herein do not necessarily state or reflect those of the U.S. Government or any agency thereof. 


\title{
ECAR 4951 BSU-8242 3 DPA As-Run Physics Analysis
}

\author{
Jason V Brookman
}

July 2020

\section{Idaho National Laboratory Idaho Falls, Idaho 83415}

\author{
http://www.inl.gov
}

\author{
Prepared for the \\ U.S. Department of Energy \\ Under DOE Idaho Operations Office \\ Contract DE-AC07-05ID14517
}




\begin{tabular}{|ll|c|}
\hline 1. & Effective Date & $07 / 02 / 2020$ \\
\hline 2. & $\begin{array}{l}\text { Does this ECAR involve a } \\
\text { Safety SSC? }\end{array}$ & Yes \\
\hline 3. & $\begin{array}{l}\text { Safety SSC Determination } \\
\text { Document ID }\end{array}$ & NA \\
\hline 4. & SSC ID & NA \\
\hline 5. & Project No. & 31418 \\
\hline 6. & Engineering Job (EJ) No. & NA \\
\hline 7. & Building & NA \\
\hline 8. & Site Area & NA \\
\hline
\end{tabular}
Professional Engineer's Stamp
N/A

See LWP-10010 for requirements (per LWP-10010 Sec 4.1, par. cc)

9. Objective/Purpose:

The purpose of this report is to document the as-run physics analysis for the Boise State University (BSU-8242) experiment in the A6, A7 and A8 positions, correlating with the 3 DPA specimens irradiated in Cycles 164A, 164B, and 166A. In this paper the heat rate, flux, fluence, and DPA for all specimens are scaled to the appropriate as-run lobe powers for an accurate assessment and description of the irradiated materials. Source terms per capsule are provided, and a bounded source term per specimen type is given on a per gram basis. Verified and validated MCNP full-core models were used for the neutronics analysis.

10. If revision, please state the reason and list sections and/or pages being affected:

Revision 1.

The planned positions for capsule '400C 3 DPA (X)' and capsule '400C 3 DPA (Y)', position A-7 and position $A-8$, respectively, were swapped during irradiation across all 3 cycles: capsule '400C 3 DPA (X)' was irradiated in the A-8 position, while capsule '400C 3 DPA (Y)' was irradiated in the A-7 position. This irradiation assembly required updating the following tables: Table 10 and Table 11 heating rates; Table 13 and Table 14 neutron flux and fluence; Table 16 and Table 17 displacements per atom; Table 18 decay heat; Table 20 and Table 21 source terms. Additionally, the 'Conclusions/Recommendations' section of the report on page 1 was updated to reflect the changes. Table 1 was also updated. Lastly, Table 22, Table 23, and Table 24 were added to the report to detail the source terms in grams.

See ATTACHMENT B for the original analysis request form, and ATTACHMENT $C$ for the analysis request form in support of a Revision 1. Also provided in 'Attachment $C$ ' is the information from Reactor Engineering detailing the irradiation issues that required an updated analysis and revision to the ECAR. 
11. Conclusions/Recommendations:

An MCNP full core physics model was used to calculate the as-run heat rates, flux values, fluences, and DPA for each test specimen within the BSU-8242 experiment. The heating rates ranged from 6.67 to $17.37 \frac{\mathrm{W}}{\mathrm{g}}$ in position A-6 (300C 3 DPA Capsule), depending on the material and axial location; the heating rates ranged from 5.14 to $16.38 \frac{\mathrm{W}}{\mathrm{g}}$ in the A-7 position (400C 3 DPA $(Y)$ ), and the heating rates in the A-8 position (400C 3 DPA $(X)$ ) ranged from 5.25 to 9.81 $\frac{W}{g}$. The average neutron flux experienced by the BSU-8242 specimens in position A-6 was $\sim 9.08 \times 10^{14} \frac{\mathrm{n}}{\mathrm{cm}^{2} \cdot \mathrm{s}}$, while the average fluence was $\sim 1.47 \times 10^{22} \frac{\mathrm{n}}{\mathrm{cm}^{2}}$; The average neutron flux in position A-7 was $\sim 8.31 \times 10^{14} \frac{\mathrm{n}}{\mathrm{cm}^{2} \cdot \mathrm{s}}$, while the average fluence was $\sim 1.35 \times 10^{22} \frac{\mathrm{n}}{\mathrm{cm}^{2}}$; The average neutron flux in position A-8 was $\sim 8.65 \times 10^{14} \frac{\mathrm{n}}{\mathrm{cm}^{2} \cdot \mathrm{s}}$, while the average fluence was $\sim 1.40 \times 10^{22} \frac{\mathrm{n}}{\mathrm{cm}^{2}}$. The average DPA experienced by the specimens in the A-6 position was calculated to be 4.65, with the average being 4.32 DPA in the A-7 position, and 3.97 DPA in the A-8 position.

The heat rates are reported to support the thermal analysis in assessing the irradiation temperatures each test specimen experienced. The fluence and DPA values are reported to support PIE. In support of PIE, see excel attachment concomitant with this ECAR, which provides the bounding source terms for each specimen material on a per gram basis. 


\section{CONTENTS}

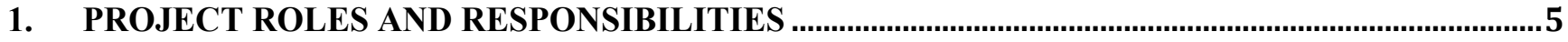

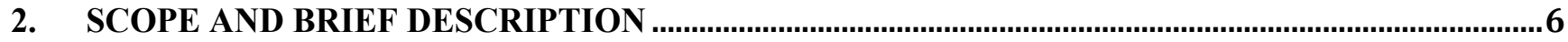

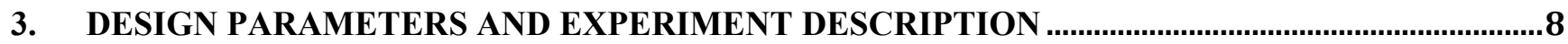

4. RESULTS OF LITERATURE SEARCHES AND OTHER BACKGROUND DATA......................... 20

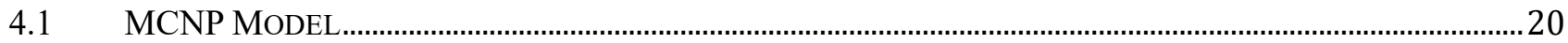

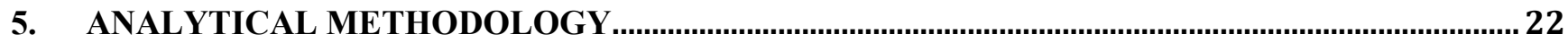

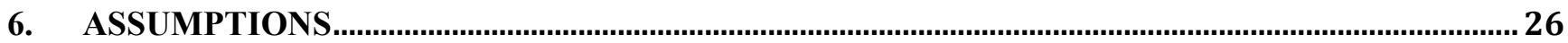

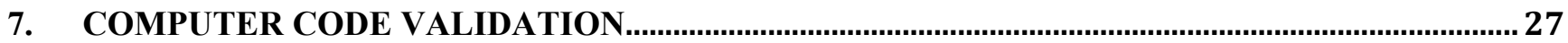

TABLE 8. COMPUTER CONFIGURATIONS FOR INL QUALIFIED MCNP AND ORIGEN2

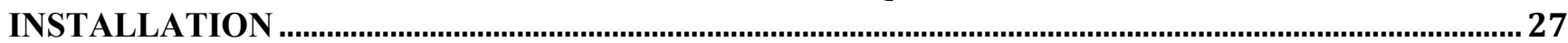

8. DISCUSSION/ANALYSIS

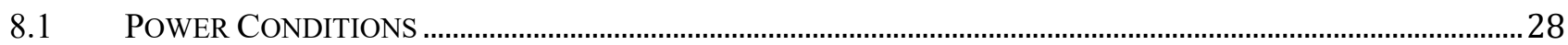

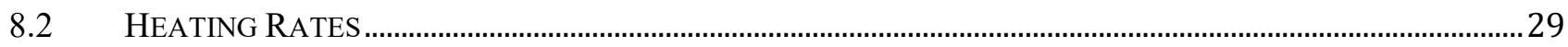

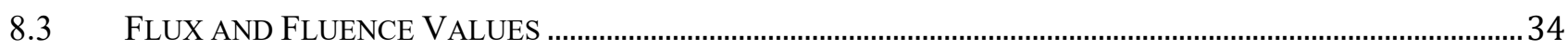

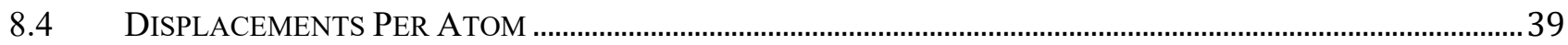

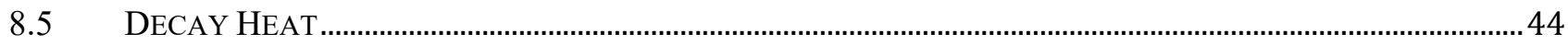

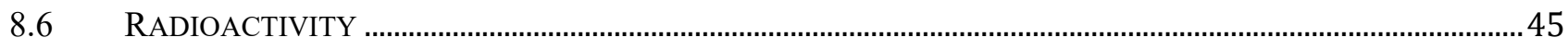

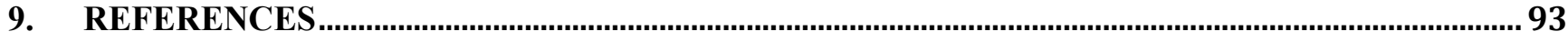

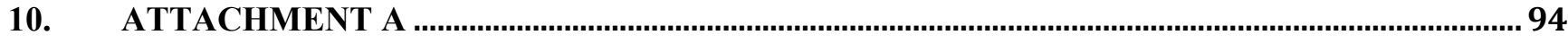

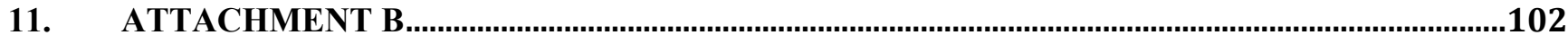

12. ATTACHMENT C …...................................................................................................................................103

\section{FIGURES}

Figure 1. Cross-Section of ATR with the A-6, A-7, AND A-8 POSITIONS SHOWn........................... 8

Figure 2. BSU-8242 CAPSULE DESIGN \& SPECIFICATION (DIMENSIONS ARE GIVEN IN INCHES) ............ 11

FIGURE 3. BSU-8242 CAPSULE LENGTH AND IDENTIFICATION (DIMENSIONS ARE GIVEN IN INCHES). .... 12

Figure 4. BSU-8242 CENTRAL FIXTURE OVERVIEW FOR ALL CAPSULES ........................................ 13

Figure 5. BSU-8242 SPECIMEN DIMENSIONS (DIMENSIONS ARE GIVEN IN INCHES): A) COMPACT TEST

(CT) SPecimen; B) Transmission Electron Microscope Disc (TEM) SPECimen; C) USU HF-

Al TEM SPECIMEN; D) Round TENSILE (RT) SPECIMEN

\section{TABLES}

TABLE 1. OVERVIEW OF THE BSU-8242 EXPERIMENT..................................................................... 9

TABle 2. Chemical Composition of BSU-8242 SPeCimen Alloys (WT/O \%) [11] ........................ 10

Table 3. Weight Percent of Elemental Content Per HF-Al Specimen type [12] ....................... 10

TABLE 4. SPECIMEN ORGANIZATION ACROSS THE 7 CAPSULES COMPOSING THE BSU-8242 EXPERIMENT.

TABLE 5. BSU-8242 CYCLE IRRADIATION OVERVIEW [1] ......................................................... 19

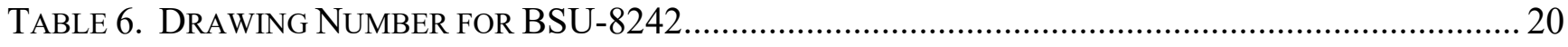


TABLE 7. INL QUALIFIED ANALYSIS SOFTWARE, VERSION, AND EA ID. 27

TABLE 8. COMPUTER CONFIGURATIONS FOR INL QUALIFIED MCNP AND ORIGEN2 INSTALLATION... 27

TABLE 9. POSITION A-6 SPECIMEN HEATING RATES OF THE UPPER BSU-8242-300 ${ }^{\circ} \mathrm{C} / 3$ DPA CAPSULE ANALYZED AT A CENTER LOBE POWER OF 19.7/21.9/22.1 MW PER THE THREE CYCLES.

TABle 10. POSITION A-7 SPECIMEN HEATING RATES OF THE LOWER BSU-8242-300 ${ }^{\circ} \mathrm{C} / 3$ DPA (Y) CAPSUlE ANALYZED AT A CENTER LOBE POWER OF 19.7/21.9/22.1 MW PER THE THREE CYCLES. . 30

TABLE 11. POSITION A-8 SPECIMEN HEATING RATES OF THE UPPER BSU-8242-400 C/3 DPA (X) CAPSUlE ANALYZED AT A CENTER LOBE POWER OF 19.7/21.9/22.1 MW PER THE THREE CYCLES. . 31

TABLE 12. POSITION A-6 SPECIMEN NEUTRON FLUX AND FLUENCE VALUES OF THE BSU-8242-300 ${ }^{\circ} \mathrm{C} / 3$ DPA CAPSUlE ANALYZED AT A CENTER LOBE POWER OF 19.7/21.9/22.1 MW PER THE THREE CYCLES.

TABLE 13. POSITION A-7 SPECIMEN NEUTRON FLUX AND FLUENCE VALUES OF THE BSU-8242-400 ${ }^{\circ} \mathrm{C} / 3$ DPA (Y) CAPSUle ANALYZED AT A CENTER LOBE POWER OF 19.7/21.9/22.1 MW PER THE THREE CYCLES.

TABLE 14. Position A-8 SPECIMEN NEUTRON FLUX AND FLUENCE VALUES OF THE BSU-8242-400 ${ }^{\circ} \mathrm{C} / 3$ DPA (X) CAPSUle ANALYZED AT A CENTER LOBE POWER OF 19.7/21.9/22.1 MW PER THE THREE CYCLES.

TABLE 15. Position A-6 SPECIMEN DPA VALUES OF THE BSU-8242-300 ${ }^{\circ} \mathrm{C} / 3$ DPA CAPSULE ANALYZED AT A CENTER LOBE POWER OF 19.7/21.9/22.1 MW PER THE THREE CYCLES.

TABle 16. Position A-7 SPECIMEN DPA VAlues OF THE BSU-8242-400 C/3 DPA (Y) CAPSULE ANALYZED AT A CENTER LOBE POWER OF 19.7/21.9/22.1 MW PER THE THREE CYCLES. 40

TABle 17. Position A-8 SPECIMEN DPA VAlues of THE BSU-8242-400 C/3 DPA (X) CAPSUle ANALYZED AT A CENTER LOBE POWER OF 19.7/21.9/22.1 MW PER THE THREE CYCLES.

TABle 18. DeCay Heating of the 3DPA CAPsules Post CyCle 166B IrRadiation. 44

TABLE 19. SOURCE TERMS FOR 'A6 300C 3 DPA' CAPSUlE. 45

TABLE 20. SOURCE TERMS FOR 'A7 400C 3 DPA Y' CAPSULE. 52

TABLE 21. SOURCE TERMS FOR 'A8 400C 3 DPA X' CAPSULE. 58

TABLE 22. SOURCE TERMS FOR 'A6 300C 3 DPA' CAPSULE. 64

TABLE 23. SOURCE TERMS FOR 'A7 400C 3 DPA Y' CAPSULE. 74

TABLE 24. SOURCE TERMS FOR 'A8 400C 3 DPA X' CAPSULE. 83 
1. PROJECT ROLES AND RESPONSIBILITIES

Project Role Name (Typed)

Organization Jason Brookman

C-130

C-130

Pages covered (if applicable)

Performer

Checker $^{\mathrm{a}}$

Independent Reviewer ${ }^{\mathrm{b}}$

Brian Gross

$\mathrm{N} / \mathrm{A}$

CUI Reviewer ${ }^{\mathrm{C}}$

Manager $^{\mathrm{d}}$

Requestor $^{\mathrm{e}}$

Nuclear Safety ${ }^{\mathrm{e}}$

Brian Gross

Misti Lillo

C-130

C- 130

Katie Anderson

C-601

N/A

Document Owner ${ }^{\mathrm{e}}$

Responsibilities:

a. Confirmation of completeness, mathematical accuracy, and correctness of data and appropriateness of assumptions.

b. Concurrence of method or approach. See definition, LWP-10106.

c. Concurrence with the document's markings in accordance with LWP-11202.

d. Concurrence of procedure compliance. Concurrence with method/approach and conclusion.

e. Concurrence with the document's assumptions and input information. See definition of Acceptance, LWP-10200.

NOTE: Delete or mark "N/A" for project roles not engaged. Include ALL personnel and their roles listed above in the eCR system. The list of the roles above is not all-inclusive. If needed, the list can be extended or reduced. 


\section{SCOPE AND BRIEF DESCRIPTION}

This Engineering Calculations Analysis Report (ECAR) documents the as-run results of the Advanced Test Reactor (ATR) physics analysis performed to support the irradiation of the Boise State University-8242 (BSU-8242) experiment in the A6, A7, and A8 positions of the ATR for the 3 DPA specimens. The purpose of this analysis is to calculate the following as-run data:

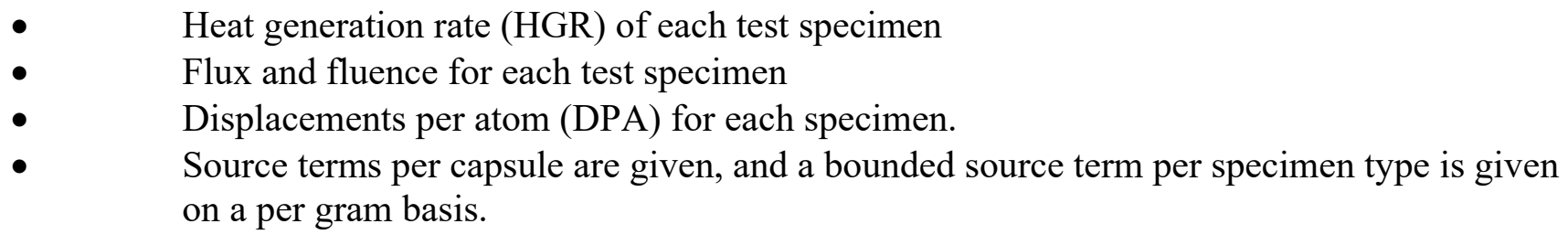

The as-run heating rates, neutron flux/fluence results, and DPA were calculated using the MCNP ATR full core model. The heating rates and fluence values were based on ATR operating conditions for the cycles of operation. Insertion of the BSU-8242 3 DPA irradiation test began on September 18, 2018 with Cycle 164B, continued through Cycle 166A, and ended irradiation with the ending of Cycle 166B on January 10, 2020.

The BSU-8242 experiment consists of seven capsules loaded with 256 material test specimens, collectively, that were irradiated in the A-6, A-7, and A-8 positions of the ATR. Four capsules with a target of 1 DPA occupied the A-7 and A-8 test positions during ATR Cycle 164A. These capsules were removed after one cycle of irradiation and three different capsules were loaded in the A-6, A-7, and A-8 test positions beginning in ATR Cycle 164B with a minimum target of 3 DPA.

BSU-8242 implemented additional flux wires and other means to measure the actual flux/exposure of the test specimens; thus, the primary function of the Monte Carlo N-Particle (MCNP) tool is to provide best estimates of the specimens irradiated for post irradiation analysis. Ultimately, the objective of BSU-8242 is to provide: 1.) a comparison of the irradiation response of five PM-HIP alloys to that of their conventionally-fabricated counterparts; 2.) an understanding of temperature functionality; and 3.) an understanding of the dose evolution of the irradiation effects.

Manufacturing processes have considerable influence over the safety and integrity of nuclear reactor vessel internal components. These processes are well-established; structural and pressure-retaining materials used in the nuclear power industry have been produced by casting, plate rolling-and-welding, forging, drawing, and extrusion, for the past 60 years. However, issues of weldability and inspectability continue to challenge the manufacture of reactor internals. Furthermore, all of these components will be subject to harsh service environments, which combine high radiation fluence, high temperature, mechanical stress, and often, corrosion potential. These conditions will accelerate material degradation and failure. Advanced fission reactor designs as well as life extensions to the existing fleet of light water reactors will exacerbate material degradation issues by increasing the duty on structural materials. Thus, ensuring the integrity of structural materials in such extreme environments is of utmost importance to the continued safety and operation of nuclear power plants.

The objective of this project is to assess the viability of using alloys manufactured by powder metallurgy and hot isostatic pressing (PM-HIP) as nuclear reactor internals, to enhance the weldability and inspectability of these components. Recently, alloys produced by PM-HIP have been developed and introduced for pressure-retaining applications in the electric power industry. These PM-HIP components exhibit good structural uniformity, no chemical segregation, superior mechanical properties, and 
enhanced weldability. PM-HIP components are produced near-net shape, which offers the distinct advantages of minimizing the need for machining and enhancing the ease of inspectability of the component. These superior properties make PM-HIP alloys potential candidates for structural materials in nuclear reactor technologies, including light water reactors (LWRs), advanced light water reactors (ALWRs), small modular reactors (SMRs), and advanced (e.g. Generation IV) reactor designs. However, little is known about the irradiation response of PM-HIP alloys, particularly in comparison to that of conventionally-manufactured alloys. Understanding these irradiation effects is the focus of this project. 


\section{DESIGN PARAMETERS AND EXPERIMENT DESCRIPTION}

The Boise State University-8242 experiment was a drop-in design irradiated for 4 cycles in the Advanced Test Reactor (ATR). Idaho National Laboratory (INL), through the Department of Energy Idaho Operations office, irradiated specimens for the Nuclear Science User Facilities (NSUF).

An MCNP snapshot depicting a cross-section of the ATR core is provided in Figure 1. Seven BSU8242 capsules loaded with 256 materials test specimens, collectively, were irradiated in the A-6, A-7, and A-8 ATR irradiation positions. Four capsules occupied the A-7 and A-8 test positions beginning in ATR Cycle 164A. These capsules were removed after one cycle of irradiation and three different capsules were loaded in the A-6, A-7, and A-8 test positions beginning in ATR Cycle 164B. The BSU-8242 capsules were not irradiated during ATR PALM cycles.

The 256 materials test specimens consisted of 28 Compact Test (CT) specimens, 48 round tensile (RT) specimens, and 180 Transmission Electron Microscope (TEM) specimens. Of the 180 TEM specimens, 36 were composed of Hf-Al alloys inserted in available space within the seven BSU-8242 capsules, intended for the NSUF sample library at HFEF. These 256 test specimens consisted of nine unique material types: six BSU-8242 materials and three Hf-Al alloys.

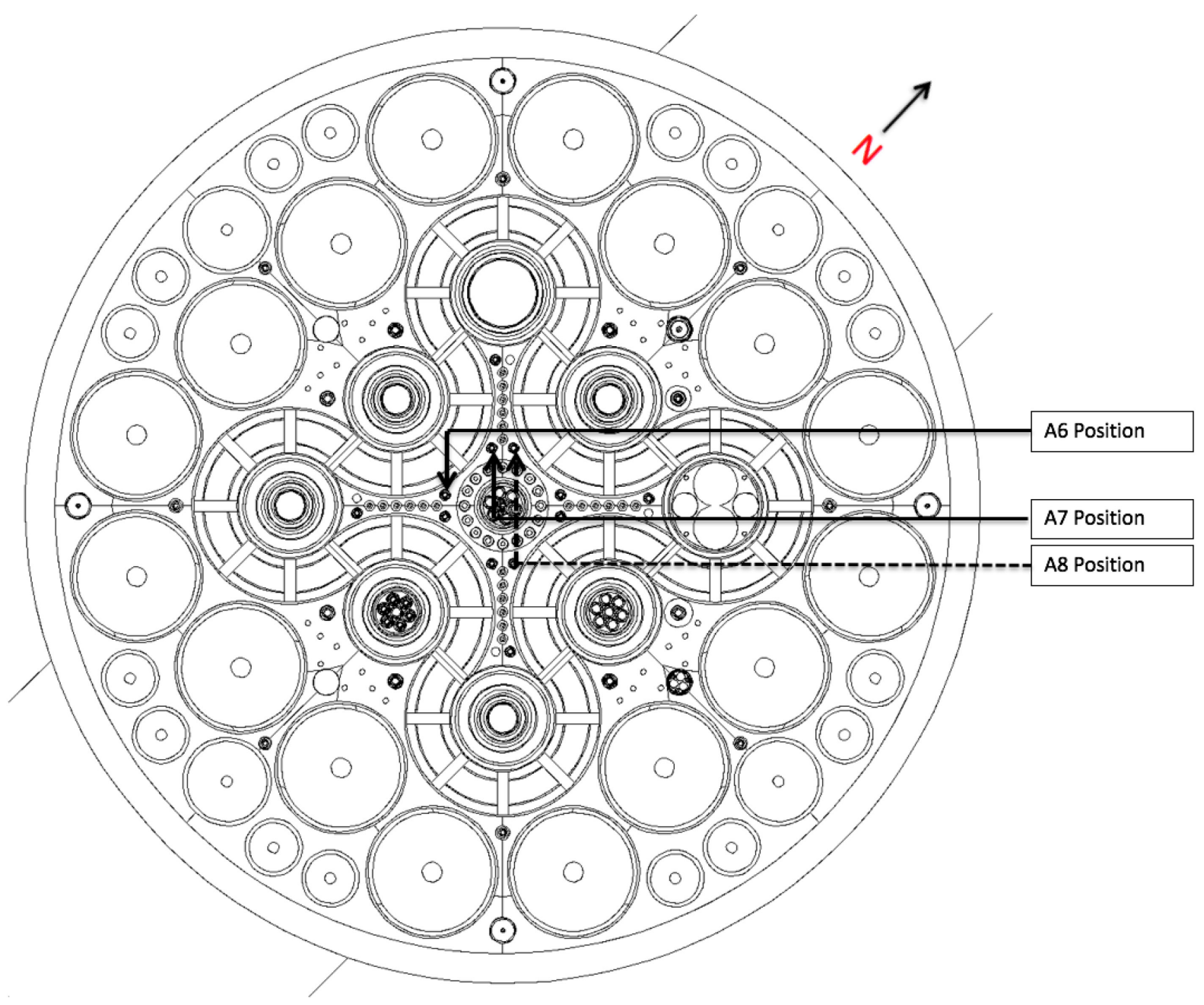

Figure 1. Cross-Section of ATR with the A-6, A-7, and A-8 positions shown. 
The BSU-8242 TEM discs were $3 \mathrm{~mm}$ in diameter and $150 \pm 50 \mu \mathrm{m}$ in thickness. The Hf-Al TEM discs were $3 \mathrm{~mm} \pm 50 \mu \mathrm{m}$ in diameter and $300 \pm 50 \mu \mathrm{m}$ thick. The round tensile specimens had a gauge length of $31.75 \mathrm{~mm} \pm 50 \mu \mathrm{m}$ and a total length of $76.2 \mathrm{~mm} \pm 50 \mu \mathrm{m}$. The CT specimens measured $10 \mathrm{~mm} \pm 50 \mu \mathrm{m} \times 9.6 \mathrm{~mm} \pm 50 \mu \mathrm{m}$ with a thickness of $4 \mathrm{~mm} \pm 50 \mu \mathrm{m}$.

An overview of the experiment and capsule descriptions can be found in Table 1. Table 2 provides the chemical composition of the 6 BSU-8242 specimen alloys, and Table 3 provides the chemical composition of the additional Hf-Al TEM specimens. Figure 2 through Figure 5 provide schematic/dimensional representations of the BSU-8242 experiment setup. A more involved designation of all specimens within each capsule is provided in Table 4. Table 5 provides the lobe powers per cycle and EFPDs for Cycles 164B-1, 166A-1, and 166B-1.

Table 1. Overview of the BSU-8242 Experiment.

\begin{tabular}{|c|c|c|c|c|}
\hline Test Train ID\# & Capsule ID & Specimen content & $\begin{array}{c}\text { Irradiation } \\
\text { Cycles }\end{array}$ & ATR position \\
\hline \multirow{2}{*}{$8242-\mathrm{A}$} & $300 \mathrm{C} / 1 \mathrm{DPA}(\mathrm{X})$ & $\begin{array}{c}\text { RT specimens: } 2 \\
\text { CT specimens: } 4 \\
\text { TEM specimens: } 21\end{array}$ & $164 \mathrm{~A}$ & A7 \\
\hline & $400 \mathrm{C} / 1 \mathrm{DPA}(\mathrm{X})$ & $\begin{array}{c}\text { RT specimens: } 8 \\
\text { CT specimens: } 4 \\
\text { TEM specimens: } 48\end{array}$ & $164 \mathrm{~A}$ & A7 \\
\hline \multirow{2}{*}{$8242-B$} & 300C/1 DPA (Y) & $\begin{array}{l}\text { RT specimens: } 2 \\
\text { CT specimens: } 4 \\
\text { TEM specimens: } 0\end{array}$ & $164 \mathrm{~A}$ & A8 \\
\hline & 400C/1 DPA (Y) & $\begin{array}{l}\text { RT specimens: } 8 \\
\text { CT specimens: } 4 \\
\text { TEM specimens: } 9\end{array}$ & $164 \mathrm{~A}$ & A8 \\
\hline $8242-\mathrm{C}$ & $300 \mathrm{C} / 3 \mathrm{DPA}$ & $\begin{array}{c}\text { RT specimens: } 8 \\
\text { CT specimens: } 4 \\
\text { TEM specimens: } 33\end{array}$ & 164B, 166A, 166B & A6 \\
\hline 8242-D & 400C/3 DPA (Y) & $\begin{array}{c}\text { RT specimens: } 10 \\
\text { CT specimens: } 4 \\
\text { TEM specimens: } 60\end{array}$ & 164B, 166A, 166B & A7 \\
\hline $8242-\mathrm{E}$ & $400 \mathrm{C} / 3 \mathrm{DPA}(\mathrm{X})$ & $\begin{array}{l}\text { RT specimens: } 10 \\
\text { CT specimens: } 4 \\
\text { TEM specimens: } 9\end{array}$ & 164B, 166A, 166B & A8 \\
\hline
\end{tabular}


BSU-8242 3 DPA As-Run Physics Analysis

Table 2. Chemical Composition of BSU-8242 Specimen Alloys (wt/o \%) [11].

\begin{tabular}{|c|c|c|c|c|c|c|}
\hline \multicolumn{7}{|c|}{ Chemical Composition of BSU-8242 Specimen Alloys } \\
\hline Element & $\mathbf{3 0 4 L}$ & $\mathbf{3 1 6 L}$ & $\mathbf{6 2 5}$ & $\mathbf{6 9 0}$ & SA 508 & Grade 91 \\
\hline$\rho$ & & & & & & \\
$\left(\mathrm{g} / \mathrm{cm}^{3}\right)$ & 8.03 & 7.99 & 8.44 & 8.19 & 7.83 & 7.81 \\
\hline $\mathrm{C}$ & 0.03 & 0.03 & 0.10 & 0.05 & 0.27 & 0.10 \\
\hline $\mathrm{Mn}$ & 2.00 & 2.00 & 0.50 & 0.50 & 0.75 & 0.46 \\
\hline $\mathrm{P}$ & 0.045 & 0.045 & 0.015 & - & 0.025 & 0.02 \\
\hline $\mathrm{S}$ & 0.03 & 0.03 & 0.015 & 0.015 & 0.025 & 0.01 \\
\hline $\mathrm{Si}$ & 0.75 & 0.75 & 0.50 & 0.50 & 0.275 & 0.37 \\
\hline $\mathrm{Cr}$ & 19 & 17 & 21.42 & 30 & 0.35 & 8.72 \\
\hline $\mathrm{Ni}$ & 10 & 12 & 57.95 & 57.975 & 0.75 & 0.40 \\
\hline $\mathrm{Al}$ & 0.10 & - & 0.40 & 0.02 & - & 0.04 \\
\hline $\mathrm{Mo}$ & - & 2.50 & 9.00 & - & 0.63 & 0.95 \\
\hline $\mathrm{N}$ & 0.10 & 0.10 & - & - & - & 0.053 \\
\hline $\mathrm{Nb}$ & - & - & 3.65 & - & - & 0.08 \\
\hline $\mathrm{Ti}$ & - & - & 0.40 & - & - & 0.01 \\
\hline $\mathrm{Ta}$ & - & - & 0.05 & - & - & - \\
\hline $\mathrm{Cu}$ & - & - & - & 0.50 & - & - \\
\hline $\mathrm{Co}$ & - & - & 1.00 & - & - & - \\
\hline $\mathrm{V}$ & - & - & - & - & 0.05 & 0.215 \\
\hline $\mathrm{Zr}$ & - & - & - & - & - & - \\
\hline $\mathrm{Fe}$ & 67.945 & 65.545 & 5.00 & 10.44 & 96.875 & 88.572 \\
\hline
\end{tabular}

Table 3. Weight Percent of Elemental Content per Hf-Al Specimen type [12].

Material Specification

\begin{tabular}{|c|c|c|c|}
\hline Element & $\mathbf{2 0 \%}$ Hf-Al & $\mathbf{2 8 . 4 \%}$ Hf-Al & $\mathbf{3 6 . 5 \%}$ Hf-Al \\
\hline $\left.\begin{array}{c}\rho \\
\left(\mathrm{g} / \mathrm{cm}^{3}\right.\end{array}\right)$ & 3.43 & 3.74 & 3.95 \\
\hline $\mathrm{Al}$ & 74.76 & 67.04 & 60.7 \\
\hline $\mathrm{Zr}$ & 0.885 & 0.885 & 0.885 \\
\hline $\mathrm{Hf}$ & 24.36 & 32.08 & 38.42 \\
\hline
\end{tabular}




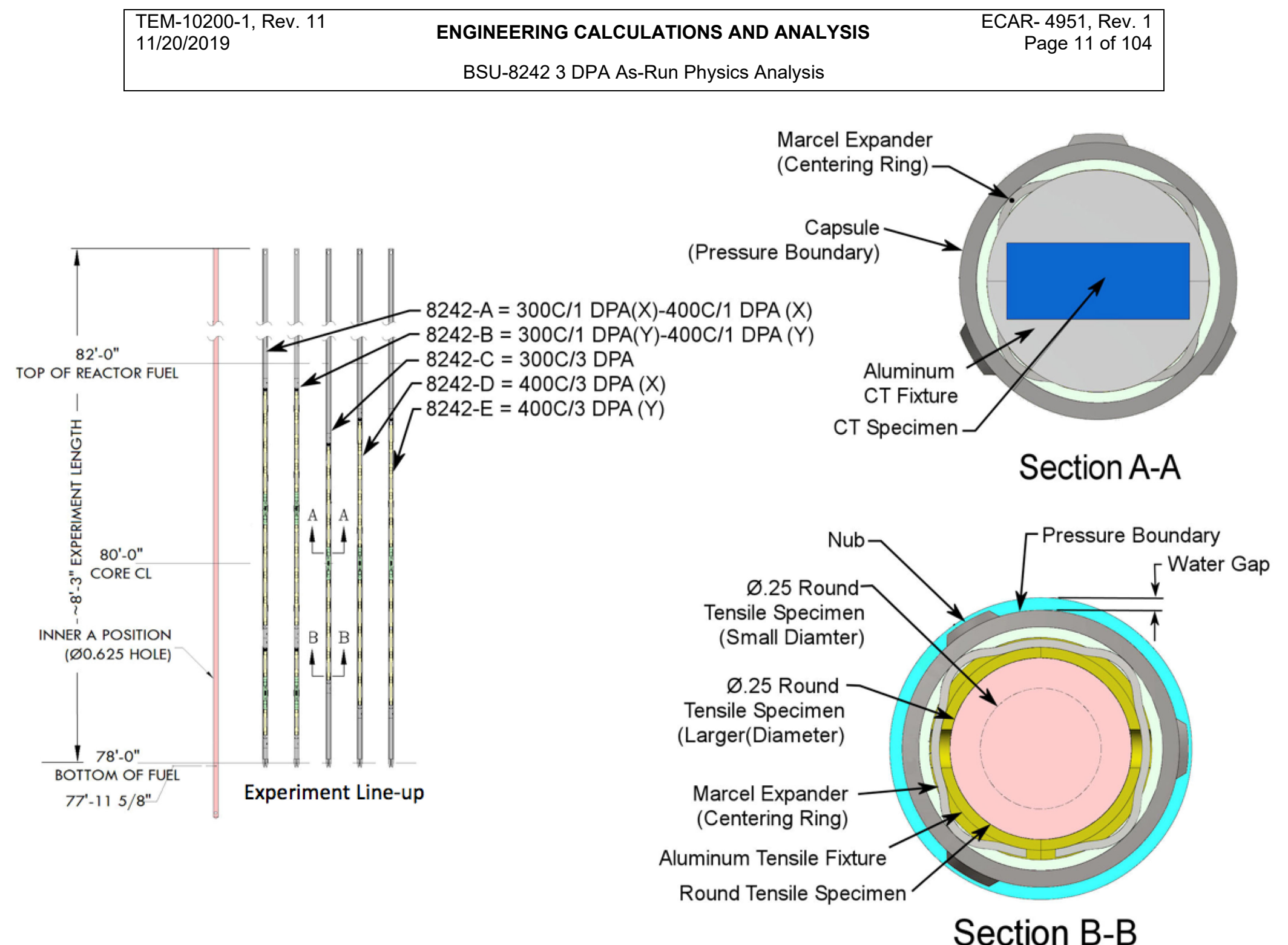

Figure 2. BSU-8242 Capsule Design \& Specification (dimensions are given in inches) 


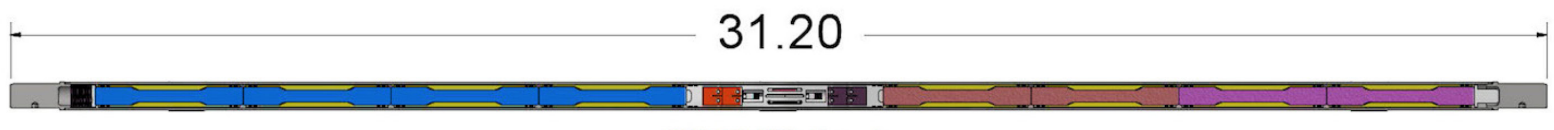

300. C 3 DPA Capsule
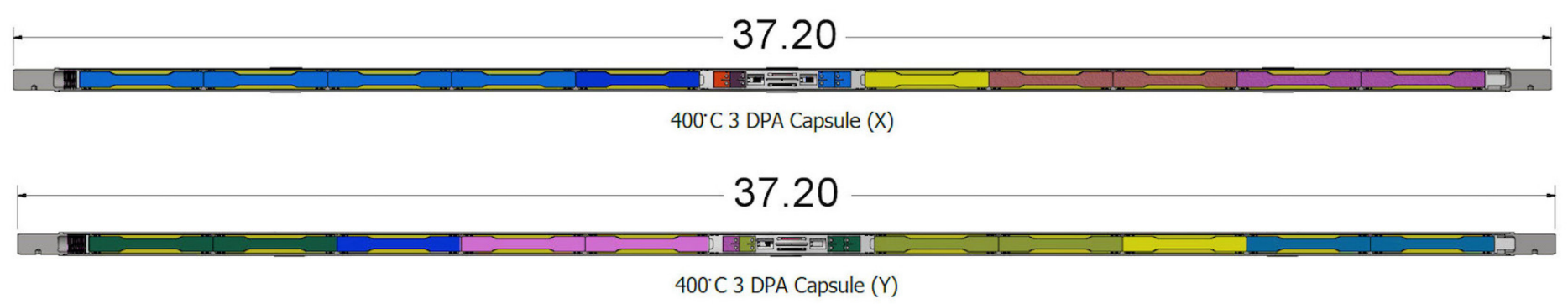

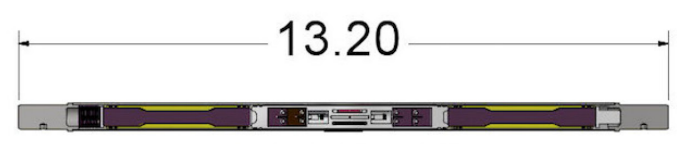

$300^{\circ}$ C 1 DPA Capsule (X)

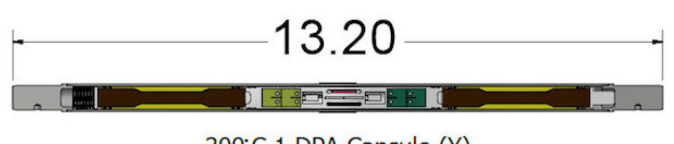

$300^{\circ}$ C 1 DPA Capsule (Y)
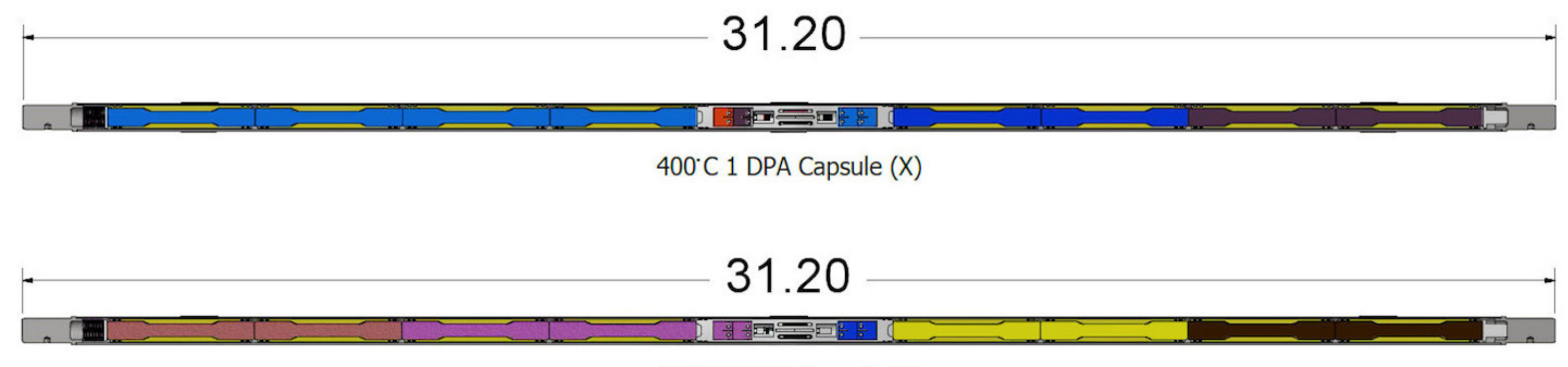

400. C 1 DPA Capsule (Y)

Figure 3. BSU-8242 Capsule Length and Identification (dimensions are given in inches). 


\begin{tabular}{|lcr|}
\hline $\begin{array}{l}\text { TEM-10200-1, Rev. } 11 \\
11 / 20 / 2019\end{array}$ & ENGINEERING CALCULATIONS AND ANALYSIS & ECAR- 4951, Rev. 1 \\
& Page 13 of 104 \\
\hline
\end{tabular}

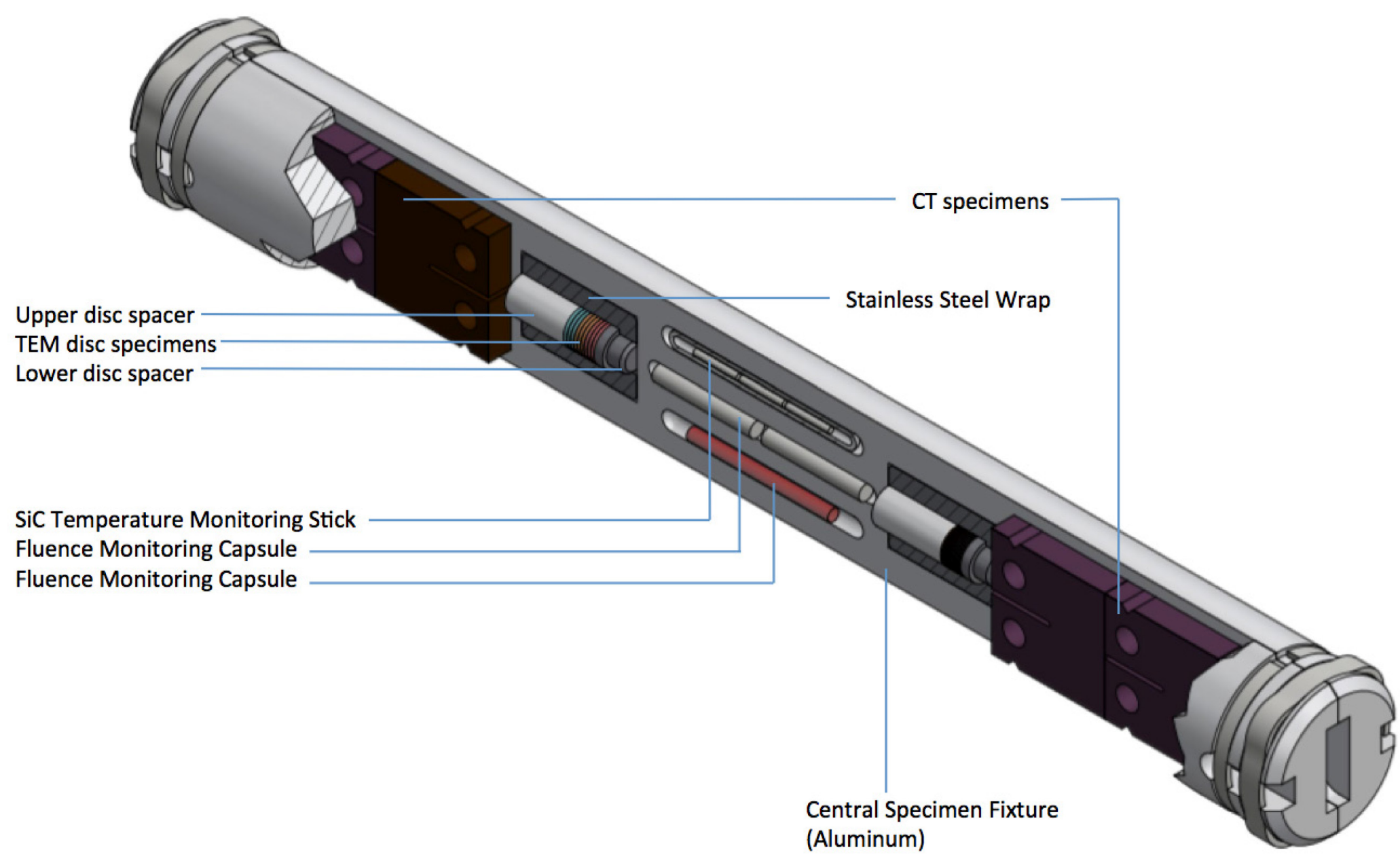

Figure 4. BSU-8242 Central Fixture Overview for all Capsules 


\begin{tabular}{|lrr|}
\hline TEM-10200-1, Rev. 11 & ENGINEERING CALCULATIONS AND ANALYSIS & ECAR- 4951, Rev. 1 \\
$11 / 20 / 2019$ & Page 14 of 104 \\
& \\
\hline
\end{tabular}

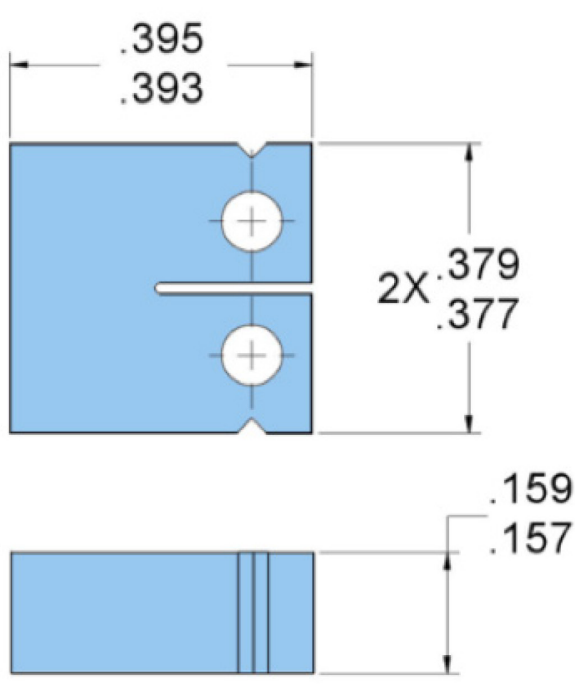

A

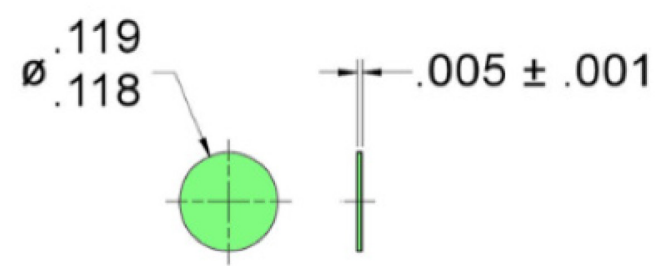

B

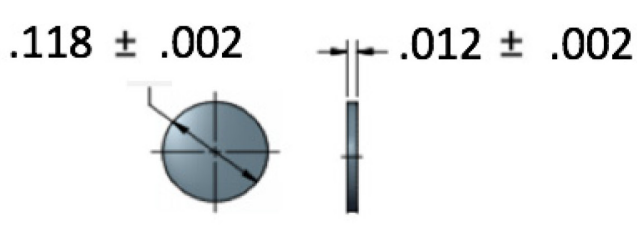

C

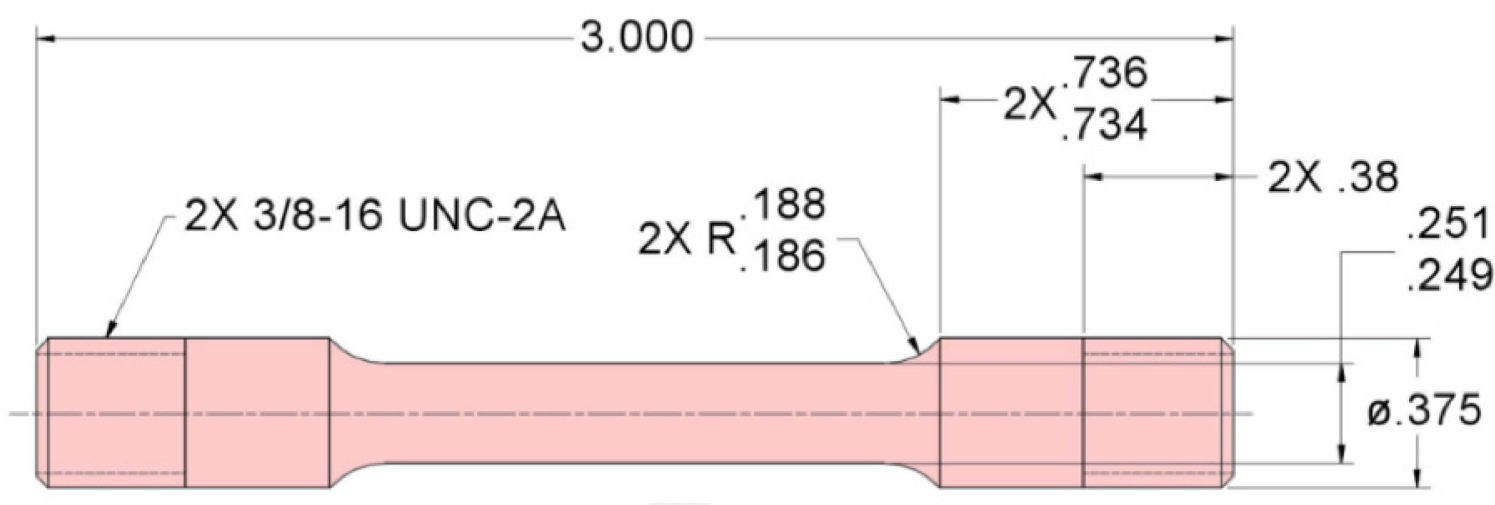

D

Figure 5. BSU-8242 Specimen Dimensions (dimensions are given in inches): A) Compact Test (CT) Specimen; B) Transmission Electron Microscope Disc (TEM) Specimen; C) USU Hf-Al TEM specimen; D) Round Tensile (RT) Specimen 
Table 4. Specimen organization across the 7 capsules composing the BSU-8242 experiment.

\begin{tabular}{|c|c|c|c|c|c|c|}
\hline \multicolumn{7}{|c|}{ Specimen Organization } \\
\hline 400C/ 1DPA (X) & 300C/ 1DPA (X) & 400C/ 1DPA (Y) & 300C/ 1DPA (Y) & 300C/ 3DPA & 400C/ 3DPA (X) & 400C/ 3DPA (Y) \\
\hline Top of Capsule & Top of Capsule & Top of Capsule & Top of Capsule & Top of Capsule & Top of Capsule & Top of Capsule \\
\hline 460. RT-625 & 127. RT-SA508 & 521. RT-625 & 206. RT-508 & 345. RT-690 & 674. RT-690 & 723. RT-304 \\
\hline 459. RT-625 & 126. CT-SA508 & 520. RT-625 & 205. CT-316L & 344. RT-690 & 673. RT-690 & 722. RT-304 \\
\hline 458. RT-690 & 125. CT-SA508 & 519. RT-690 & 204. CT-316L & 343. RT-625 & 672. RT-625 & 721. RT-91 \\
\hline 457. RT-690 & 124. TEM-USU 20 & 518. RT-690 & 203. CT-316L & 342. RT-625 & 671. RT-625 & 720. RT-316 \\
\hline 456. СТ-625 & 123. TEM-USU 20 & 517. CT-690 & 202. CT-316L & 341. СТ-625 & 670. RT-91 & 719. RT-316 \\
\hline 455. CT-625 & 122. TEM-USU 20 & 516. CT-690 & 201. RT-508 & 340. CT-625 & 669. CT-625 & 718. CT-690 \\
\hline 454. TEM-625 & 121. TEM-USU 28 & 515. TEM-USU 20 & Bottom of Capsule & 339. TEM-USU 20 & 668. CT-625 & 717. CT-316 \\
\hline 453. TEM-625 & 120. TEM-USU 28 & 514. TEM-USU 20 & & 338. TEM-USU 20 & 667. TEM-91 & 716. TEM-USU 36 \\
\hline 452. TEM-625 & 119. TEM-USU 28 & 513. TEM-USU 20 & & 337. TEM-USU 20 & 666. TEM-91 & 715. TEM-USU 36 \\
\hline 451. TEM-625 & 118. TEM-USU 36 & 512. TEM-USU 28 & & 336. TEM-USU 28 & 665. TEM-91 & 714. TEM-USU 36 \\
\hline 450. TEM-625 & 117. TEM-USU 36 & 511. TEM-USU 28 & & 335. TEM-USU 28 & 664. TEM-91 & 713. TEM-USU 28 \\
\hline 449. TEM-625 & 116. TEM-USU 36 & 510. TEM-USU 28 & & 334. TEM-USU 28 & 663. TEM-91 & 712. TEM-USU 28 \\
\hline 448. TEM-625 & 115. TEM-SA508 & 509. TEM-USU 36 & & 333. TEM-USU 36 & 662. TEM-91 & 711. TEM-USU 28 \\
\hline 447. TEM-625 & 114. TEM-SA508 & 508. TEM-USU 36 & & 332. TEM-USU 36 & 661. TEM-304L & 710. TEM-USU 20 \\
\hline 446. TEM-625 & 113. TEM-SA508 & 507. TEM-USU 36 & & 331. TEM-USU 36 & 660. TEM-304L & 709. TEM-USU 20 \\
\hline 445. TEM-625 & 112. TEM-SA508 & 506. CT-91 & & 330. TEM-690 & 659. TEM-304L & 708. TEM-USU 20 \\
\hline 444. TEM-625 & 111. TEM-SA508 & 505. CT-91 & & 329. TEM-690 & 658. TEM-304L & 707. CT-316 \\
\hline 443. TEM-625 & 110. TEM-SA508 & 504. RT-91 & & 328. TEM-690 & 657. TEM-304L & 706. CT-316 \\
\hline 442. TEM-SA508 & 109. TEM-SA508 & 503. RT-91 & & 327. TEM-690 & 656. TEM-304L & 705. RT-316 \\
\hline 441. TEM-SA508 & 108. TEM-SA508 & 502. RT-508 & & 326. TEM-690 & 655. TEM-304L & 704. RT-316 \\
\hline 440. TEM-SA508 & 107. TEM-SA508 & 501. RT-508 & & 325. TEM-690 & 654. TEM-304L & 703. RT-91 \\
\hline 439. TEM-SA508 & 106. TEM-SA508 & Bottom of Capsule & & 324. TEM-625 & 653. TEM-304L & 702. RT-304 \\
\hline 438. TEM-SA508 & 105. TEM-SA508 & & & 323. TEM-625 & 652. TEM-304L & 701. RT-304 \\
\hline 437. TEM-SA508 & 104. TEM-SA508 & & & 322. TEM-625 & 651. TEM-304L & Bottom of Capsule \\
\hline
\end{tabular}




\begin{tabular}{lrr}
\hline TEM-10200-1, Rev. 11 & ENGINEERING CALCULATIONS AND ANALYSIS & ECAR- 4951, Rev. 1 \\
$11 / 20 / 2019$ & Page 16 of 104 \\
& BSU-8242 3 DPA As-Run Physics Analysis
\end{tabular}

Table 4. Specimen organization across the 7 capsules composing the BSU-8242 experiment.

\begin{tabular}{|c|c|c|c|c|c|c|}
\hline \multicolumn{7}{|c|}{ Specimen Organization } \\
\hline 400C/ 1DPA (X) & 300C/ 1DPA (X) & 400C/ 1DPA (Y) & 300C/ 1DPA (Y) & 300C/ 3DPA & 400C/ 3DPA (X) & 400C/ 3DPA (Y) \\
\hline Top of Capsule & Top of Capsule & Top of Capsule & Top of Capsule & Top of Capsule & Top of Capsule & Top of Capsule \\
\hline 436. TEM-SA508 & 103. CT-SA508 & & & 321. TEM-625 & 650. TEM-304L & \\
\hline 435. TEM-SA508 & 102. CT-SA508 & & & 320. TEM-625 & 649. TEM-316L & \\
\hline 434. TEM-SA508 & 101. RT-SA508 & & & 319. TEM-625 & 648. TEM-316L & \\
\hline 433. TEM-SA508 & Bottom of Capsule & & & 318. TEM-690 & 647. TEM-316L & \\
\hline 432. TEM-SA508 & & & & 317. TEM-690 & 646. TEM-316L & \\
\hline 431. TEM-SA508 & & & & 316. TEM-690 & 645. TEM-316L & \\
\hline 430. TEM-91 & & & & 315. TEM-690 & 644. TEM-316L & \\
\hline 429. TEM-91 & & & & 314. TEM-690 & 643. TEM-316L & \\
\hline 428. TEM-91 & & & & 313. TEM-690 & 642. TEM-316L & \\
\hline 427. TEM-91 & & & & 312. TEM-625 & 641. TEM-316L & \\
\hline 426. TEM-91 & & & & 311. TEM-625 & 640. TEM-316L & \\
\hline 425. TEM-91 & & & & 310. TEM-625 & 639. TEM-316L & \\
\hline 424. TEM-91 & & & & 309. TEM-625 & 638. TEM-316L & \\
\hline 423. TEM-91 & & & & 308. TEM-625 & 637. TEM-91 & \\
\hline 422. TEM-91 & & & & 307. TEM-625 & 636. TEM-91 & \\
\hline 421. TEM-91 & & & & 306. СТ- 625 & 635. TEM-91 & \\
\hline 420. TEM-91 & & & & 305. CT-625 & 634. TEM-91 & \\
\hline 419. TEM-91 & & & & 304. RT-625 & 633. TEM-91 & \\
\hline 418. TEM-690 & & & & 303. RT-625 & 632. TEM-91 & \\
\hline 417. TEM-690 & & & & 302. RT-690 & 631. TEM-690 & \\
\hline 416. TEM-690 & & & & 301. RT-690 & 630. TEM-690 & \\
\hline 415. TEM-690 & & & & Bottom of Capsule & 629. TEM-690 & \\
\hline 414. TEM-690 & & & & & 628. TEM-690 & \\
\hline 413. TEM-690 & & & & & 627. TEM-690 & \\
\hline 412. TEM-690 & & & & & 626. TEM-690 & \\
\hline
\end{tabular}




\begin{tabular}{lcr|}
$\begin{array}{l}\text { TEM-10200-1, Rev. } 11 \\
11 / 20 / 2019\end{array}$ & ENGINEERING CALCULATIONS AND ANALYSIS & ECAR- 4951, Rev. 1 \\
& Page 17 of 104 \\
\end{tabular}

Table 4. Specimen organization across the 7 capsules composing the BSU-8242 experiment.

\begin{tabular}{|c|c|c|c|c|c|c|}
\hline \multicolumn{7}{|c|}{ Specimen Organization } \\
\hline 400C/ 1DPA (X) & 300C/ 1DPA (X) & 400C/ 1DPA (Y) & 300C/ 1DPA (Y) & 300C/ 3DPA & 400C/ 3DPA (X) & 400C/ 3DPA (Y) \\
\hline Top of Capsule & Top of Capsule & Top of Capsule & Top of Capsule & Top of Capsule & Top of Capsule & Top of Capsule \\
\hline 411. TEM-690 & & & & & 625. TEM-690 & \\
\hline 410. TEM-690 & & & & & 624. TEM-690 & \\
\hline 409. TEM-690 & & & & & 623. TEM-690 & \\
\hline 408. TEM-690 & & & & & 622. TEM-690 & \\
\hline 407. TEM-690 & & & & & 621. TEM-690 & \\
\hline 406. CT-690 & & & & & 620. TEM-690 & \\
\hline 405. CT-690 & & & & & 619. TEM-625 & \\
\hline 404. RT-91 & & & & & 618. TEM-625 & \\
\hline 403. RT-91 & & & & & 617. TEM-625 & \\
\hline 402. RT-SA508 & & & & & 616. TEM-625 & \\
\hline 401. RT-SA508 & & & & & 615. TEM-625 & \\
\hline Bottom of Capsule & & & & & 614. TEM-625 & \\
\hline & & & & & 613. TEM-625 & \\
\hline & & & & & 612. TEM-625 & \\
\hline & & & & & 611. TEM-625 & \\
\hline & & & & & 610. TEM-625 & \\
\hline & & & & & 609. TEM-625 & \\
\hline & & & & & 608. TEM-625 & \\
\hline & & & & & 607. CT-690 & \\
\hline & & & & & 606. СТ-690 & \\
\hline & & & & & 605. RT-91 & \\
\hline & & & & & 604. RT-625 & \\
\hline & & & & & 603. RT-625 & \\
\hline & & & & & 602. RT-690 & \\
\hline & & & & & 601. RT-690 & \\
\hline
\end{tabular}




\begin{tabular}{lrr}
\hline TEM-10200-1, Rev. 11 & ENGINEERING CALCULATIONS AND ANALYSIS & ECAR- 4951, Rev. 1 \\
$11 / 20 / 2019$ & Page 18 of 104 \\
& BSU-8242 3 DPA As-Run Physics Analysis
\end{tabular}

Table 4. Specimen organization across the 7 capsules composing the BSU-8242 experiment.

\begin{tabular}{c|c|c|c|c|c|c|c}
\hline \multicolumn{7}{c}{ Specimen Organization } \\
\hline 400C/ 1DPA (X) & 300C/ 1DPA (X) & 400C/ 1DPA (Y) & 300C/ 1DPA (Y) & 300C/ 3DPA & 400C/ 3DPA (X) & 400C/ 3DPA (Y) \\
\hline Top of Capsule & Top of Capsule & Top of Capsule & Top of Capsule & Top of Capsule & Top of Capsule & Top of Capsule \\
\hline
\end{tabular}


Table 5. BSU-8242 cycle irradiation overview [1].

\begin{tabular}{|c|c|c|c|c|}
\hline \multirow{3}{*}{ Parameter } & \multicolumn{4}{|c|}{ Cycle $^{1,2}$} \\
\hline & \multirow{2}{*}{$\begin{array}{c}1 \mathrm{DPA} \\
164 \mathrm{~A}-1 \\
\end{array}$} & \multicolumn{3}{|c|}{3 DPA } \\
\hline & & 164B-1 & 166A-1 & 166B-1 \\
\hline NW (MW) & 20.0 & 19.5 & 19.9 & 19.9 \\
\hline NE (MW) & 16.0 & 16.4 & 16.9 & 16.9 \\
\hline $\mathrm{C}(\mathrm{MW})^{1}$ & 19.4 & 19.7 & 21.9 & 22.1 \\
\hline SW (MW) & 22.4 & 23.1 & 25.8 & 25.9 \\
\hline SE (MW) & 25.7 & 25.1 & 25.6 & 25.0 \\
\hline Total Core (MW) & 103.5 & 103.8 & 110.1 & 109.8 \\
\hline EFPDs & 54.9 & 64.1 & 62.5 & 61.2 \\
\hline Post Outage (days) & 32 & 189 & 34 & -- \\
\hline
\end{tabular}

1 BSU-8242 data was scaled to the center lobe powers.

2 ATR lobe powers per cycle are given in reference [1]. 
BSU-8242 3 DPA As-Run Physics Analysis

\section{RESULTS OF LITERATURE SEARCHES AND OTHER BACKGROUND DATA}

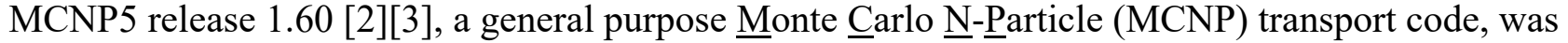
used to model and evaluate the neutron and photon heating of the BSU-8242 components, the neutron flux and fluence values, and the DPA of each test specimen.

\subsection{MCNP Model}

The MCNP models describing the BSU-8242 experiment in three of the ATR inner-A positions are based on the drawings listed in Table 6 . The BSU-8242 test was axially centered (by position) about the ATR core mid-plane within each MCNP model.

\begin{tabular}{|c|c|c|}
\hline \multicolumn{3}{|r|}{ Table 6. Drawing Number for BSU-8242. } \\
\hline $\begin{array}{l}\text { Drawing } \\
\text { Number }\end{array}$ & Revision & Drawing Title \\
\hline 605765 & 3 & $\begin{array}{l}\text { ATR NSUF BOISE EXPERIMENT } 8242 \text { INBOARD A CAPSULE } \\
\text { COMPONENT ASSEMBLIES AND DETAILS }\end{array}$ \\
\hline 605766 & 0 & $\begin{array}{l}\text { ATR NSUF BOISE EXPERIMENT } 8242 \text { 300C-1 DPA X INBOARD } \\
\text { A CAPSULE CT AND TEM SPECIMEN ASSEMBLY }\end{array}$ \\
\hline 605767 & 2 & $\begin{array}{l}\text { ATR NSUF BOISE EXPERIMENT } 8242 \text { 300C-1 DPA Y INBOARD } \\
\text { A CAPSULE CT AND TEM SPECIMEN ASSEMBLY }\end{array}$ \\
\hline 605768 & 1 & $\begin{array}{l}\text { ATR NSUF BOISE EXPERIMENT } 8242 \text { 300C-3 DPA INBOARD A } \\
\text { CAPSULE CT AND TEM SPECIMEN ASSEMBLY }\end{array}$ \\
\hline 605770 & 0 & $\begin{array}{l}\text { ATR NSUF BOISE EXPERIMENT } 8242 \text { 400C-1 DPA Y INBOARD } \\
\text { A CAPSULE CT AND TEM SPECIMEN ASSEMBLY }\end{array}$ \\
\hline 605771 & 2 & $\begin{array}{l}\text { ATR NSUF BOISE EXPERIMENT } 8242 \text { 400C-1 DPA X INBOARD } \\
\text { A CAPSULE CT AND TEM SPECIMEN ASSEMBLY }\end{array}$ \\
\hline 605772 & 1 & $\begin{array}{l}\text { ATR NSUF BOISE EXPERIMENT } 8242 \text { 400C-3 DPA Y INBOARD } \\
\text { A CAPSULE CT AND TEM SPECIMEN ASSEMBLY }\end{array}$ \\
\hline 605773 & 3 & $\begin{array}{l}\text { ATR NSUF BOISE EXPERIMENT } 8242 \text { INBOARD A CAPSULE } \\
\text { DRAWING TREE }\end{array}$ \\
\hline 605774 & 3 & $\begin{array}{l}\text { ATR NSUF BOISE EXPERIMENT } 8242 \text { SPECIMEN ASSEMBLY } \\
\text { AND DETAILS }\end{array}$ \\
\hline 605775 & 0 & $\begin{array}{l}\text { ATR NSUF BOISE EXPERIMENT } 8242 \text { INBOARD A CAPSULE } \\
\text { TENSILE SPECIMEN ASSEMBLIES }\end{array}$ \\
\hline 605776 & 1 & $\begin{array}{l}\text { ATR NSUF BOISE EXPERIMENT } 8242 \text { 300C-1 DPA X INBOARD } \\
\text { A CAPSULE ASSEMBLY }\end{array}$ \\
\hline 605777 & 1 & $\begin{array}{l}\text { ATR NSUF BOISE EXPERIMENT } 8242 \text { 300C-3 DPA INBOARD } \\
\text { A CAPSULE ASSEMBLY }\end{array}$ \\
\hline 605778 & 1 & $\begin{array}{l}\text { ATR NSUF BOISE EXPERIMENT } 8242 \text { 300C-1 DPA Y INBOARD } \\
\text { A CAPSULE ASSEMBLY }\end{array}$ \\
\hline 605824-1 & 2 & $\begin{array}{l}\text { ATR BSU-8242 EXPERIMENT INBOARD A CAPSULE 300C/1 } \\
\text { DPA (X) AND 400C/1 DPA (X) TEST TRAIN ASSEMBLY }\end{array}$ \\
\hline $605825-1$ & 2 & $\begin{array}{l}\text { ATR BSU-8242 EXPERIMENT INBOARD A CAPSULE 300C/1 } \\
\text { DPA (Y) AND 400C/1 DPA (Y) TEST TRAIN ASSEMBLY }\end{array}$ \\
\hline $605826-1$ & 3 & $\begin{array}{l}\text { ATR BSU-8242 EXPERIMENT INBOARD A CAPSULE 300C/3 } \\
\text { DPA TEST TRAIN ASSEMBLY }\end{array}$ \\
\hline
\end{tabular}


Table 6. Drawing Number for BSU-8242.

\begin{tabular}{|l|c|l|}
\hline \multicolumn{2}{|c|}{ Table 6. Drawing Number for BSU-8242. } \\
\hline $\begin{array}{l}\text { Drawing } \\
\text { Number }\end{array}$ & Revision & Drawing Title \\
\hline $605827-1$ & 3 & $\begin{array}{l}\text { ATR BSU-8242 EXPERIMENT INBOARD A CAPSULE 400C/3 } \\
\text { DPA (X) TEST TRAIN ASSEMBLY }\end{array}$ \\
\hline $605828-1$ & 2 & $\begin{array}{l}\text { ATR BSU-8242 EXPERIMENT INBOARD A CAPSULE 400C/3 } \\
\text { DPA (Y) TEST TRAIN ASSEMBLY }\end{array}$ \\
\hline 605829 & 1 & $\begin{array}{l}\text { ATR NSUF BOISE EXPERIMENT 8242 INBOARD A CAPSULE } \\
\text { TEST TRAIN INSTALLATION }\end{array}$ \\
\hline 120421 & 11 & $\begin{array}{l}\text { ATR OUTBOARD AND INBOARD “A” POSITION SPECIMEN } \\
\text { FILLER PLUGS }\end{array}$ \\
\hline 602380 & 1 & UTAH STATE UNIVERSITY SPECIMEN DETAILS \\
\hline
\end{tabular}




\section{ANALYTICAL METHODOLOGY}

MCNP is used to calculate the neutron flux values, heat generation rates and DPA in the BSU-8242 experiment. MCNP reports the flux tallies in units of $1 / \mathrm{cm}^{2}$ per source neutron. The following normalization factors are used to calculate the neutron flux and the heat generation rates, as well as the DPA, from the MCNP tallies.

\section{Neutron Flux Normalization Factor}

MCNP reports tally results normalized per source particle. The MCNP type 4 flux tally results are used to generate neutron flux input values for BSU-8242. The MCNP tally type 4 (for neutrons) has units of neutrons $/ \mathrm{cm}^{2}$ per source neutron. The neutron flux conversion factor (NFCF) is defined by equation (1).

$$
\begin{aligned}
& N F C F=\left(\frac{2.43 \text { fission neutrons }}{\text { fission }}\right)\left(\frac{\text { fission }}{200 \mathrm{MeV}}\right)\left(\frac{6.24151 \times 10^{18} \mathrm{MeV}}{M W_{\text {Core Power }} \cdot \mathrm{S}}\right) \\
& N F C F=7.583 \times 10^{16} \frac{\text { fission neutrons }}{M W_{\text {Core Power }} \cdot \mathrm{S}}
\end{aligned}
$$

The neutron flux values are calculated using the MCNP tally type 4 results, the NFCF, and the ATR core power. The neutron flux is calculated using Equation (2).

$$
\begin{aligned}
& \phi_{\text {neutron }}=\left(\text { type } 4 \text { tally } \frac{\text { neutrons }}{\mathrm{cm}^{2}-\text { fission neutron }}\right)(7.583 \\
&\left.\times 10^{16} \frac{\text { fission neutrons }}{M W_{\text {core power }}-S}\right)(\text { Core Power } M W) \\
& \phi_{\text {neutron }}=(f 4)\left(7.583 \times 10^{16}\right)(\text { Core Power }) \frac{\text { neutrons }}{\mathrm{cm}^{2}-\mathrm{s}}
\end{aligned}
$$

\section{Neutron/Prompt Gamma Heating Normalization Factor (NHNF)}

MCNP reports tally results normalized per source particle. The MCNP type 6 energy deposition tally results or type 7 fission energy deposition tally results are used to calculate heat generation rates. The MCNP tally type 6 has units of MeV/g per source particle (fission neutron for prompt neutron, gamma heating, and fission heating). The heating normalization factor (HNF) is defined by equation (3).

$$
\begin{aligned}
& H N F=\left(\frac{2.43 \text { fission neutrons }}{\text { fission }}\right)\left(\frac{\text { fission }}{200 \mathrm{MeV}}\right)\left(\frac{1 \times 10^{6} \mathrm{~W}}{1 \mathrm{MW}}\right) \\
& H N F=1.215 \times 10^{4} \frac{\text { fission neutrons } \cdot \mathrm{W}}{\mathrm{MW} \cdot \mathrm{MeV}}
\end{aligned}
$$

The heat generation rate values are calculated using the MCNP tally type 6 results, the HNF, and the ATR core power. Prompt neutron and gamma heating rates (PHR) are calculated using equation (4).

PHR $=\left(\right.$ type 6 tally $\left.\frac{\text { MeV }}{g \cdot f \text { ission neutron }}\right)\left(1.215 \times 10^{4} \frac{\text { fission neutrons } \cdot W}{M W \cdot M e V}\right)($ Core Power $M W)$ 
PHR $=(f 6)(H N F)($ Core Power $) \frac{W}{g}$

\section{Delayed Fission Product Gamma Heating Normalization Factor}

MCNP reports tally results normalized per source particle. The heating tallies have units of MeV/g per fission neutron. The MCNP type 6 energy deposition tally results are used to calculate delayed gamma heat generation rates. The MCNP tally type 6 has units of $\mathrm{MeV} / \mathrm{g}$ per source particle (per delayed fission product gamma for delayed fission product gamma heating). The delayed photon heating normalization factor (DPHNF) is defined by equation (5) using 8.9603 delayed fission photons per fission [4][5].

$\mathrm{DPHNF}=\left(\frac{8.9603 \text { delayed } \text { photons }}{\text { fission }}\right)\left(\frac{\text { fission }}{200 \mathrm{MeV}}\right)\left(\frac{1 \times 10^{6} \mathrm{~W}}{M W}\right)$

$\mathrm{DPHNF}=4.480 \times 10^{4} \frac{\text { delayed photons } \cdot \mathrm{W}}{M W_{\text {core power }} \cdot \mathrm{MeV}}$

The heat generation rate values are calculated using the MCNP tally type 6 or type 7 results, the HNF, and the ATR core power. Delayed fission product heating rate (DHR) is calculated using equation (6).

$$
\begin{aligned}
& D H R=\left(\text { type } 6 \text { tally } \frac{M e V}{g \cdot \text { source photon }}\right)\left(4.480 \times 10^{4} \frac{\text { delayed photons } \cdot W}{M W \cdot M e V}\right)(\text { Core Power } M W) \\
& D H R=(f 6)(\text { DPHNF })(\text { Core Power }) \frac{W}{g}
\end{aligned}
$$

\section{Displacements per Atom}

To evaluate radiation damage, a fundamental parameter that characterizes lattice displacement events is required. DPA has been used to compare radiation damage by different radiation sources. It is a damage-based exposure unit and represents the number of atoms displaced from their normal lattice sites as a result of energetic particle bombardment. Displacements per atom (DPA) is used to quantify radiation damage (the number of times that an atom is displaced for a given fluence). DPA is a standard measure for computing neutron- and gamma-induced radiation damage of materials that are found in radiation prone environments. It essentially provides the correlation between mechanical structural damage properties associated with the level of radiation that the material has encountered over the time span inside the neutron radiation environment [6].

The DPA rate in a material is estimated by using a tally multiplier card with a standard flux tally in MCNP5: the neutron flux is calculated as a function of energy on a material and it is combined with the DPA cross-section. The tally multiplier card applied to a flux tally calculates the reaction rate that is defined as the quantity:

$C \int \phi(\mathrm{E}) \mathrm{R}_{\mathrm{m}}(\mathrm{E}) \mathrm{dE}$ 
Where,

$C=$ multiplicative constant

$\phi(E)=$ energy dependent flux

$R_{m}(E)=$ energy dependent reaction rate of interest (ENDF/B-VII damage cross-section)

The quantity:

$\int \phi(E) R_{m}(E) d E$

is the total damage energy rate for the material. Assigning the quantity $\eta / 2 \mathrm{E}_{\mathrm{d}}$ to the constant $\mathrm{C}$ in Equation 6 results in:

$D P A$ rate $=\frac{\eta}{2 E_{d}} \int \phi(\mathrm{E}) \mathrm{R}_{\mathrm{m}}(\mathrm{E}) \mathrm{dE}$

$\phi(E) R_{m}(E)=$ total damage energy

The DPA cross-section would be calculated by:

$\sigma_{D P A}=\frac{\int \phi(E) R(E) d E}{\int \phi(E) d E}$

The resulting cross section has units of $\mathrm{MeV}$-barns per atom.

This is done by employing the cross-section processing code system NJOY [7] to produce microscopic DPA cross-section data. This is due to the fact that NJOY data is based on detailed physical nuclear interactions. It is from this nuclear data that it is possible to generate DPA cross sections, which are accurate for 0 to $20 \mathrm{MeV}$, making it applicable for LWRs. The NJOY nuclear data processing code is regarded as the golden standard to process evaluated nuclear data files [8]. The damage cross section is produced in the HEATR function within NJOY99, which is used to add heat-production (kerma) and DPA cross sections [9]. To load the DPA cross section into the simulation the MT=444 reaction must be called with the input file [6]. 
DPA is calculated by folding MCNP5-provided displacement cross sections with the energy dependent neutron particle spectrum. Where the MT=444 card is employed (reaction number for the damage cross sections), the cross sections are folded into the flux tally data, producing tally data that are in units of $\frac{\text { MeV-barns }}{\mathrm{cm}^{2}-\text { source particles }}$. The following method is then used to calculate the DPA:

$D P A$ rate $\left(\frac{d p a}{s}\right)=\left[F M n(E) \cdot \sigma(E)_{\text {damage cross-section }}\right]_{\text {tally }} \cdot \eta \cdot \frac{1}{2 E_{d}}$.

Neutron Flux Normalization Factor · Core Power · Unit Conversion Factor

where the tally is in units of $\frac{M e V-\text { barns }}{\mathrm{cm}^{2}-\text { source particles }} ; \eta$ is the efficiency, usually taken to be 0.8 ; $E_{\mathrm{d}}$ is the weighted average cutoff energy of the specimen material, calculated by an atomic weighted average of the material (the typical stainless steel value employed is $40 \times 10^{-6} \mathrm{MeV}$, which is the value used for this analysis for both the rodlet and capsule); the NFCF is defined above $(7.583 \times$ $\left.10^{16} \frac{\text { fission neutrons }}{M W_{\text {Core Power }} \text { s }}\right)$; the unit conversion factor is $1 \times 10^{-24} \frac{\mathrm{cm}^{2}}{\mathrm{~b}}$. The DPA rate is then multiplied by the irradiation time to give the displacements per atom. 


\section{ASSUMPTIONS}

The following assumptions are used in this analysis:

1. The core operates with the lobe powers specified in Table 5 for cycles 164B-1, 166A-1, and 166B-1. The lobe powers were taken from Reference [1] (also see ATTACHMENT A for a listing of the average ATR lobe power values per cycle). Heat rates, fluxes, and DPA results are scaled to the center lobe power. Changes in the center lobe power should be accounted for in the HGR and DPA results by multiplying by the ratio of the new center lobe power over the analyzed center lobe power.

2. Heating rate values reported for all capsules include energy deposition from prompt neutrons, prompt gammas, and delayed fission product gammas.

3. The ATR fuel model consists of three radial and seven axial zones to represent the ATR fuel elements.

4. An atomic displacement energy of $40 \mathrm{eV}$ (for calculating DPA) was applied to every material analyzed in this ECAR, aside from the USU samples. This value is appropriate for stainless steels and is applicable to the BSU-8242 specimens. The DPA for the USU specimens (of differing hafnium amounts) were calculated using the following cutoff energies: $32.7 \mathrm{eV}, 34.5 \mathrm{eV}$, and 36.0 $\mathrm{eV}$ for the $20 \%, 28.4 \%$, and $36.5 \% \mathrm{Hf}-\mathrm{Al}$ samples, respectively. The cutoff energy for each of the USU specimens was calculated by an elemental weighted average approach.

5. This analysis used the same density for each of the three types of material fabrication methods (PMHIP, cast, and forged).

6. The Hf-Al samples are composed of hafnium-aluminum alloy "microspheres" ( 3 aluminum to 1 hafnium by atom percent) dispersed within an aluminum matrix at three differing volume percentages $(20 \%, 28.4 \%$, and $36.5 \%)$. 


\section{COMPUTER CODE VALIDATION}

Release 1.60 of MCNP (References [2] [3]), a general purpose $\underline{\text { Monte }} \underline{\text { Carlo }} \underline{\mathrm{N}}$-P्article transport code, was used to perform the calculations documented in this ECAR. The ENDF/B-VI and ENDF/B-VII cross-section data libraries [8] [9] were used.

MCNP is archived in the INL Enterprise Architecture (EA) Repository as qualified scientific and engineering analysis software. Table 7 lists the version and CTM UUID's for the release of MCNP and ORIGEN used in this ECAR.

Table 7. INL Qualified Analysis Software, Version, and EA ID.

\begin{tabular}{|c|c|c|}
\hline Code Name & Version & CTM UUID \\
\hline MCNP & 5 (Release 1.60) & $\begin{array}{c}\text { 66DB1859-B150-4898- } \\
\text { AF8C-374D01595284 }\end{array}$ \\
\hline ORIGEN & 2.2 & $\begin{array}{c}\text { 83837D31-E66F-489D- } \\
\text { 950D-A09AECFBA8CD }\end{array}$ \\
\hline
\end{tabular}

MCNP Release 1.60 and ORIGEN Version 2.2 have been V\&V'd for use by the INL Neutronics Analysis Group as documented in Reference [14].

The computer configuration summarized in Table 8 below was used to perform the MCNP calculations documented in this ECAR.

Table 8. Computer Configurations for INL Qualified MCNP and ORIGEN2 Installation

\begin{tabular}{|l|c|l|}
\hline \multicolumn{1}{|c|}{ Computer } & \multicolumn{1}{|c|}{ Processor/Hardware } & \multicolumn{1}{c|}{ Operating System } \\
\hline Falcon is a 34992-core & Two Intel Xeon E5-2695 v4 CPUs: & SUSE Linux \\
SGI ICE X distributed & $\bullet$ Broadwell Chipset & Enterprise Server 12 \\
memory cluster with & $\bullet 18$ Cores per CPU & Service Pack 4 \\
121 TB of total & $\bullet 2.10 \mathrm{GHz}$ & Operating System \\
memory. & $\bullet 128 \mathrm{~GB}$ of RAM & 3.0.101-108.35- \\
& FDR InfiniBand Interconnect & default \\
\hline
\end{tabular}




\section{DISCUSSION/ANALYSIS}

The MCNP full core physics model was used to calculate the as-run heat rates, flux and fluence values, and DPA for each of the test specimens within each of the three capsules corresponding to the 3 DPA specimens of the BSU-8242 experiment. The as-run flux values were used to determine the fluence for the experiment based on the irradiation time across all three cycles.

The heating rate values are given below (8.2 Heating Rates); the flux and fluence data follow (8.3 Flux and Fluence Values); finally, the DPA values are tabled (8.4 Displacements Per Atom).

The heating rates ranged from 6.67 to $17.37 \frac{\mathrm{W}}{\mathrm{g}}$ in position A-6 (300C 3 DPA Capsule), depending on the material and axial location; the heating rates ranged from 5.14 to $16.38 \frac{\mathrm{W}}{\mathrm{g}}$ in the A-7 position (400C 3 DPA (Y)), and the heating rates in the A-8 position (400C 3 DPA (X)) ranged from 5.25 to $9.81 \frac{\mathrm{W}}{\mathrm{g}}$. The average neutron flux experienced by the BSU-8242 specimens in position A-6 was $\sim 9.08 \times 10^{14} \frac{\mathrm{n}}{\mathrm{cm}^{2} \cdot \mathrm{s}}$, while the average fluence was $\sim 1.47 \times 10^{22} \frac{\mathrm{n}}{\mathrm{cm}^{2}}$; The average neutron flux in position A-7 was $\sim 8.31 \times 10^{14} \frac{n}{\mathrm{~cm}^{2} \cdot \mathrm{s}}$, while the average fluence was $\sim 1.35 \times 10^{22} \frac{\mathrm{n}}{\mathrm{cm}^{2}}$; The average neutron flux in position A-8 was $\sim 8.65 \times 10^{14} \frac{\mathrm{n}}{\mathrm{cm}^{2} \cdot \mathrm{s}}$, while the average fluence was $\sim 1.40 \times 10^{22} \frac{\mathrm{n}}{\mathrm{cm}^{2}}$. The average DPA experienced by the specimens in the A- 6 position was calculated to be 4.65 , with the average being 4.32 DPA in the A-7 position, and 3.97 DPA in the A-8 position.

The heat rates are reported to support the thermal analysis in assessing the irradiation temperatures each test specimen experienced. The fluence and DPA values are reported to support PIE. In support of PIE, see excel attachment concomitant with this ECAR, which provides the bounding source terms for each specimen material on a per gram basis.

\subsection{Power Conditions}

The analysis for each cycle employed a model describing an average fuel core loading and average control settings. The as-run flux values were used to determine the fluences for the experiment based on the irradiation time. The values were obtained by scaling the MCNP data to the average Center lobe power obtained from each cycle [1].

The lobe powers along with the EFPD values and the full-core powers are provided in Table 5. 


\subsection{Heating Rates}

The following three tables (Tables 9-11) provide the heating rates for each test specimen within each of the 3 DPA capsules. The heat generation rates (HGRs) for the 3 DPA BSU-8242 specimens have been calculated using nominal ATR core power conditions. The HGRs for each test specimen in the BSU8242 capsules have been calculated at nominal ATR core power conditions using MCNP. These HGR results are scaled to the cycle specific center lobe powers listed in Table 5, which correspond to the asrun operating powers. The HGR results include neutron and gamma heating, as well as delayed gamma heating. Delayed gamma heating was explicitly calculated using a photon only (mode p) MCNP model.

Table 9. Position A-6 specimen heating rates of the upper BSU-8242-300 ${ }^{\circ} \mathrm{C} / 3 \mathrm{DPA}$ capsule analyzed at a Center Lobe Power of 19.7/21.9/22.1 MW per the three cycles.

\begin{tabular}{|l|c|c|c|c|c|}
\hline \multicolumn{7}{|c|}{ A-6 Position [300C 3 DPA] } \\
\hline Specimen/Material & $\begin{array}{c}\text { Specimen } \\
\text { Number }\end{array}$ & $\begin{array}{c}\text { Distance } \\
\text { from Core } \\
\text { Midplane } \\
\text { (cm) }\end{array}$ & $\begin{array}{c}\text { Heating } \\
{\left[\frac{W}{g}\right]} \\
\text { Cycle 1 }\end{array}$ & $\begin{array}{c}\text { Heating } \\
{\left[\frac{W}{g}\right]} \\
\text { Cycle 2 }\end{array}$ & $\begin{array}{c}\text { Heating } \\
{\left[\frac{W}{g}\right]} \\
\text { Cycle 3 }\end{array}$ \\
\hline RT-690 PM-HIP & 345 & 31.75 & 6.67 & 7.42 & 7.49 \\
\hline RT-690 PM-HIP & 344 & 24.13 & 7.54 & 8.38 & 8.46 \\
\hline RT-625 PM-HIP & 343 & 16.51 & 8.48 & 9.42 & 9.51 \\
\hline RT-625 PM-HIP & 342 & 8.89 & 8.94 & 9.94 & 10.03 \\
\hline CT-625 PM-HIP & 341 & 3.22 & 9.39 & 10.44 & 10.54 \\
\hline CT-625 PM-HIP & 340 & 3.22 & 9.36 & 10.40 & 10.50 \\
\hline TEM-USU-300-3-36 & 339 & 1.39 & 14.60 & 16.23 & 16.38 \\
\hline TEM-USU-300-3-36 & 338 & 1.37 & 14.49 & 16.11 & 16.26 \\
\hline TEM-USU-300-3-36 & 337 & 1.36 & 14.29 & 15.89 & 16.04 \\
\hline TEM-USU-300-3-28 & 336 & 1.34 & 13.13 & 14.60 & 14.73 \\
\hline TEM-USU-300-3-28 & 335 & 1.33 & 13.24 & 14.72 & 14.86 \\
\hline TEM-USU-300-3-28 & 334 & 1.31 & 13.49 & 14.99 & 15.13 \\
\hline TEM-USU-300-3-20 & 333 & 1.30 & 12.03 & 13.37 & 13.50 \\
\hline TEM-USU-300-3-20 & 332 & 1.28 & 12.14 & 13.50 & 13.63 \\
\hline TEM-USU-300-3-20 & 331 & 1.27 & 11.84 & 13.16 & 13.28 \\
\hline TEM-690 Forged & 330 & -1.38 & 8.75 & 9.73 & 9.82 \\
\hline TEM-690 Forged & 329 & -1.39 & 8.73 & 9.71 & 9.80 \\
\hline TEM-690 Forged & 328 & -1.41 & 8.87 & 9.86 & 9.95 \\
\hline TEM-690 Forged & 327 & -1.42 & 8.65 & 9.62 & 9.71 \\
\hline TEM-690 Forged & 326 & -1.44 & 8.56 & 9.51 & 9.60 \\
\hline TEM-690 Forged & 325 & -1.45 & 8.65 & 9.61 & 9.70 \\
\hline TEM-625 Forged & 324 & -1.47 & 9.22 & 10.25 & 10.35 \\
\hline TEM-625 Forged & 323 & -1.48 & 9.20 & 10.23 & 10.33 \\
\hline TEM-625 Forged & 322 & -1.50 & 9.38 & 10.42 & 10.52 \\
\hline TEM-625 Forged & 321 & -1.51 & 9.27 & 10.31 & 10.40 \\
\hline
\end{tabular}


ENGINEERING CALCULATIONS AND ANALYSIS

BSU-8242 3 DPA As-Run Physics Analysis
ECAR- 4951, Rev. 1

Page 30 of 104

\begin{tabular}{|l|c|c|c|c|c|}
\hline \multicolumn{7}{|c|}{ A-6 Position [300C 3 DPA] } \\
\hline Specimen/Material & $\begin{array}{c}\text { Specimen } \\
\text { Number }\end{array}$ & $\begin{array}{c}\text { Distance } \\
\text { from Core } \\
\text { Midplane } \\
\text { (cm) }\end{array}$ & $\begin{array}{c}\text { Heating } \\
{\left[\frac{W}{g}\right]} \\
\text { Cycle 1 }\end{array}$ & $\begin{array}{c}\text { Heating } \\
{\left[\frac{W}{g}\right]} \\
\text { Cycle 2 }\end{array}$ & $\begin{array}{c}\text { Heating } \\
{\left[\frac{W}{g}\right]} \\
\text { Cycle 3 }\end{array}$ \\
\hline TEM-625 Forged & 320 & -1.53 & 9.20 & 10.23 & 10.33 \\
\hline TEM-625 Forged & 319 & -1.54 & 9.29 & 10.33 & 10.43 \\
\hline TEM-690 PM-HIP & 318 & -1.56 & 8.83 & 9.81 & 9.91 \\
\hline TEM-690 PM-HIP & 317 & -1.57 & 8.87 & 9.86 & 9.96 \\
\hline TEM-690 PM-HIP & 316 & -1.59 & 8.86 & 9.85 & 9.94 \\
\hline TEM-690 PM-HIP & 315 & -1.60 & 8.70 & 9.67 & 9.76 \\
\hline TEM-690 PM-HIP & 314 & -1.62 & 8.66 & 9.63 & 9.72 \\
\hline TEM-690 PM-HIP & 313 & -1.63 & 8.68 & 9.65 & 9.75 \\
\hline TEM-625 PM-HIP & 312 & -1.65 & 9.44 & 10.50 & 10.60 \\
\hline TEM-625 PM-HIP & 311 & -1.66 & 9.38 & 10.43 & 10.53 \\
\hline TEM-625 PM-HIP & 310 & -1.68 & 9.30 & 10.34 & 10.44 \\
\hline TEM-625 PM-HIP & 309 & -1.69 & 9.24 & 10.27 & 10.37 \\
\hline TEM-625 PM-HIP & 308 & -1.71 & 9.30 & 10.34 & 10.44 \\
\hline TEM-625 PM-HIP & 307 & -1.72 & 9.33 & 10.37 & 10.47 \\
\hline CT-625 Cast & 306 & -3.22 & 9.40 & 10.45 & 10.55 \\
\hline CT-625 Cast & 305 & -3.22 & 9.38 & 10.43 & 10.52 \\
\hline RT-625 Forged & 304 & -8.89 & 9.04 & 10.05 & 10.15 \\
\hline RT-625 Forged & 303 & -16.51 & 8.72 & 9.69 & 9.79 \\
\hline RT-690 Forged & 302 & -24.13 & 7.90 & 8.78 & 8.86 \\
\hline RT-690 Forged & 301 & -31.75 & 7.17 & 7.97 & 8.05 \\
\hline
\end{tabular}

Table 10. Position A-7 specimen heating rates of the lower BSU-8242-300 ${ }^{\circ} \mathrm{C} / 3$ DPA (Y) capsule analyzed at a Center Lobe Power of 19.7/21.9/22.1 MW per the three cycles.

\begin{tabular}{|l|c|c|c|c|c|}
\hline \multicolumn{7}{|c|}{ A-7 Position [400C 3DPA (Y)] } \\
\hline Specimen/Material & $\begin{array}{c}\text { Specimen } \\
\text { Number }\end{array}$ & $\begin{array}{c}\text { Distance } \\
\text { from Core } \\
\text { Midplane } \\
(\mathrm{cm})\end{array}$ & $\begin{array}{c}\text { Heating } \\
{\left[\frac{\mathrm{W}}{\mathrm{g}}\right]} \\
\text { Cycle 1 }\end{array}$ & $\begin{array}{c}\text { Heating } \\
{\left[\frac{\mathrm{W}}{g}\right]} \\
\text { Cycle 2 }\end{array}$ & $\begin{array}{c}\text { Heating } \\
{\left[\frac{\mathrm{W}}{\mathrm{g}}\right]} \\
\text { Cycle 3 }\end{array}$ \\
\hline RT-304L Forged & 723 & 39.37 & 5.14 & 5.72 & 5.77 \\
\hline RT-304L Forged & 722 & 31.75 & 6.10 & 6.78 & 6.84 \\
\hline RT-91 PM-HIP & 721 & 24.13 & 6.89 & 7.65 & 7.73 \\
\hline RT-316L PM-HIP & 720 & 16.51 & 7.55 & 8.39 & 8.47 \\
\hline RT-316L PM-HIP & 719 & 8.89 & 7.94 & 8.82 & 8.91 \\
\hline CT-690 Forged & 718 & 3.22 & 8.44 & 9.38 & 9.47 \\
\hline CT-316 Cast & 717 & 3.22 & 8.25 & 9.17 & 9.26 \\
\hline
\end{tabular}


BSU-8242 3 DPA As-Run Physics Analysis

\begin{tabular}{|l|c|c|c|c|c|}
\hline \multicolumn{7}{|c|}{ A-7 Position [400C 3DPA (Y)] } \\
\hline Specimen/Material & $\begin{array}{c}\text { Specimen } \\
\text { Number }\end{array}$ & $\begin{array}{c}\text { Distance } \\
\text { from Core } \\
\text { Midplane } \\
(\mathrm{cm})\end{array}$ & $\begin{array}{c}\text { Heating } \\
{\left[\frac{\mathrm{W}}{\mathrm{g}}\right]} \\
\text { Cycle 1 }\end{array}$ & $\begin{array}{c}\text { Heating } \\
{\left[\frac{\mathrm{W}}{\mathrm{g}}\right]} \\
\text { Cycle 2 }\end{array}$ & $\begin{array}{c}\text { Heating } \\
{\left[\frac{\mathrm{W}}{\mathrm{g}}\right]} \\
\text { Cycle 3 }\end{array}$ \\
\hline TEM-USU-400-3-36 & 716 & 1.57 & 14.60 & 16.23 & 16.38 \\
\hline TEM-USU-400-3-36 & 715 & 1.54 & 14.49 & 16.11 & 16.26 \\
\hline TEM-USU-400-3-36 & 714 & 1.51 & 14.29 & 15.89 & 16.04 \\
\hline TEM-USU-400-3-28 & 713 & 1.48 & 13.13 & 14.60 & 14.73 \\
\hline TEM-USU-400-3-28 & 712 & 1.45 & 13.24 & 14.72 & 14.86 \\
\hline TEM-USU-400-3-28 & 711 & 1.42 & 13.49 & 14.99 & 15.13 \\
\hline TEM-USU-400-3-20 & 710 & 1.39 & 12.03 & 13.37 & 13.50 \\
\hline TEM-USU-400-3-20 & 709 & 1.36 & 12.14 & 13.50 & 13.63 \\
\hline TEM-USU-400-3-20 & 708 & 1.33 & 11.84 & 13.16 & 13.28 \\
\hline CT-316 PM-HIP & 707 & -3.22 & 8.31 & 9.24 & 9.33 \\
\hline CT-316 PM-HIP & 706 & -3.22 & 8.32 & 9.25 & 9.34 \\
\hline RT-316L Forged & 705 & -8.89 & 8.01 & 8.90 & 8.99 \\
\hline RT-316L Forged & 704 & -16.51 & 7.73 & 8.60 & 8.68 \\
\hline RT-91 Cast & 703 & -24.13 & 7.20 & 8.00 & 8.08 \\
\hline RT-304L Forged & 702 & -31.75 & 6.55 & 7.28 & 7.35 \\
\hline RT-304L Forged & 701 & -39.37 & 5.71 & 6.34 & 6.40 \\
\hline
\end{tabular}

Table 11. Position A-8 specimen heating rates of the upper BSU-8242-400 ${ }^{\circ} \mathrm{C} / 3$ DPA (X) capsule analyzed at a Center Lobe Power of 19.7/21.9/22.1 MW per the three cycles.

\begin{tabular}{|l|c|c|c|c|c|}
\hline \multicolumn{7}{|c|}{ A-8 Position [Lower 400C 3DPA (X)] } \\
\hline Specimen/Material & $\begin{array}{c}\text { Specimen } \\
\text { Number }\end{array}$ & $\begin{array}{c}\text { Distance } \\
\text { from Core } \\
\text { Midplane } \\
(\mathrm{cm})\end{array}$ & $\begin{array}{c}\text { Heating } \\
{\left[\frac{\mathrm{w}}{\mathrm{g}}\right]} \\
\text { Cycle 1 }\end{array}$ & $\begin{array}{c}\text { Heating } \\
{\left[\frac{\mathrm{w}}{\mathrm{g}}\right]} \\
\text { Cycle 2 }\end{array}$ & $\begin{array}{c}\text { Heating } \\
{\left[\frac{\mathrm{W}}{\mathrm{g}}\right]} \\
\text { Cycle 3 }\end{array}$ \\
\hline RT-690 PM-HIP & 674 & 39.37 & 5.25 & 5.83 & 5.89 \\
\hline RT-690 PM-HIP & 673 & 31.75 & 6.23 & 6.92 & 6.99 \\
\hline RT-625 PM-HIP & 672 & 24.13 & 7.33 & 8.15 & 8.23 \\
\hline RT-625 PM-HIP & 671 & 16.51 & 7.92 & 8.80 & 8.89 \\
\hline RT-91 PM-HIP & 670 & 8.89 & 7.63 & 8.48 & 8.56 \\
\hline CT-625 PM-HIP & 669 & 3.22 & 8.66 & 9.63 & 9.72 \\
\hline CT-625 Cast & 668 & 3.22 & 8.70 & 9.67 & 9.77 \\
\hline TEM-91 Cast & 667 & 1.70 & 7.77 & 8.64 & 8.72 \\
\hline TEM-91 Cast & 666 & 1.69 & 7.86 & 8.73 & 8.82 \\
\hline TEM-91 Cast & 665 & 1.67 & 7.84 & 8.71 & 8.80 \\
\hline TEM-91 Cast & 664 & 1.66 & 7.94 & 8.82 & 8.91 \\
\hline
\end{tabular}


ENGINEERING CALCULATIONS AND ANALYSIS

BSU-8242 3 DPA As-Run Physics Analysis
ECAR- 4951, Rev. 1

Page 32 of 104

\begin{tabular}{|c|c|c|c|c|c|}
\hline \multicolumn{6}{|c|}{ A-8 Position [Lower 400C 3DPA (X)] } \\
\hline Specimen/Material & $\begin{array}{l}\text { Specimen } \\
\text { Number }\end{array}$ & $\begin{array}{l}\text { Distance } \\
\text { from Core } \\
\text { Midplane } \\
(\mathrm{cm})\end{array}$ & $\begin{array}{l}\text { Heating } \\
\qquad\left[\frac{W}{g}\right] \\
\text { Cycle } 1\end{array}$ & $\begin{array}{c}\text { Heating } \\
{\left[\frac{W}{g}\right]} \\
\text { Cycle } 2\end{array}$ & $\begin{array}{c}\text { Heating } \\
{\left[\frac{W}{g}\right]} \\
\text { Cycle } 3\end{array}$ \\
\hline TEM-91 Cast & 663 & 1.64 & 8.13 & 9.04 & 9.13 \\
\hline TEM-91 Cast & 662 & 1.63 & 8.02 & 8.92 & 9.00 \\
\hline TEM-304L Forged & 661 & 1.61 & 7.91 & 8.80 & 8.88 \\
\hline TEM-304L Forged & 660 & 1.60 & 7.92 & 8.80 & 8.88 \\
\hline TEM-304L Forged & 659 & 1.58 & 8.10 & 9.00 & 9.09 \\
\hline TEM-304L Forged & 658 & 1.57 & 8.02 & 8.92 & 9.00 \\
\hline TEM-304L Forged & 657 & 1.55 & 7.83 & 8.71 & 8.79 \\
\hline TEM-304L Forged & 656 & 1.54 & 7.85 & 8.73 & 8.81 \\
\hline TEM-304L Forged & 655 & 1.52 & 7.72 & 8.58 & 8.66 \\
\hline TEM-304L Forged & 654 & 1.51 & 7.79 & 8.66 & 8.74 \\
\hline TEM-304L Forged & 653 & 1.49 & 7.69 & 8.55 & 8.63 \\
\hline TEM-304L Forged & 652 & 1.48 & 7.73 & 8.59 & 8.68 \\
\hline TEM-304L Forged & 651 & 1.46 & 7.70 & 8.56 & 8.64 \\
\hline TEM-304L Forged & 650 & 1.45 & 7.84 & 8.72 & 8.80 \\
\hline TEM-316L PM-HIP & 649 & 1.43 & 8.01 & 8.90 & 8.99 \\
\hline TEM-316L PM-HIP & 648 & 1.42 & 7.92 & 8.80 & 8.89 \\
\hline TEM-316L PM-HIP & 647 & 1.40 & 7.86 & 8.74 & 8.82 \\
\hline TEM-316L PM-HIP & 646 & 1.39 & 7.85 & 8.73 & 8.81 \\
\hline TEM-316L PM-HIP & 645 & 1.37 & 7.88 & 8.76 & 8.85 \\
\hline TEM-316L PM-HIP & 644 & 1.36 & 7.89 & 8.77 & 8.85 \\
\hline TEM-316L Forged & 643 & 1.34 & 8.06 & 8.96 & 9.04 \\
\hline TEM-316L Forged & 642 & 1.33 & 8.16 & 9.07 & 9.15 \\
\hline TEM-316L Forged & 641 & 1.31 & 8.16 & 9.07 & 9.16 \\
\hline TEM-316L Forged & 640 & 1.30 & 8.23 & 9.15 & 9.24 \\
\hline TEM-316L Forged & 639 & 1.28 & 8.07 & 8.97 & 9.05 \\
\hline TEM-316L Forged & 638 & 1.27 & 8.04 & 8.94 & 9.02 \\
\hline TEM-91 PM-HIP & 637 & -1.29 & 7.86 & 8.74 & 8.82 \\
\hline TEM-91 PM-HIP & 636 & -1.30 & 7.88 & 8.76 & 8.84 \\
\hline TEM-91 PM-HIP & 635 & -1.32 & 7.94 & 8.82 & 8.91 \\
\hline TEM-91 PM-HIP & 634 & -1.33 & 7.86 & 8.73 & 8.82 \\
\hline TEM-91 PM-HIP & 633 & -1.35 & 7.91 & 8.79 & 8.88 \\
\hline TEM-91 PM-HIP & 632 & -1.36 & 7.89 & 8.78 & 8.86 \\
\hline TEM-690 Forged & 631 & -1.38 & 8.06 & 8.96 & 9.04 \\
\hline
\end{tabular}


BSU-8242 3 DPA As-Run Physics Analysis

\begin{tabular}{|c|c|c|c|c|c|}
\hline \multicolumn{6}{|c|}{ A-8 Position [Lower 400C 3DPA (X)] } \\
\hline Specimen/Material & $\begin{array}{c}\text { Specimen } \\
\text { Number }\end{array}$ & $\begin{array}{l}\text { Distance } \\
\text { from Core } \\
\text { Midplane } \\
\text { (cm) }\end{array}$ & $\begin{array}{l}\text { Heating } \\
{\left[\frac{W}{g}\right]} \\
\text { Cycle } 1\end{array}$ & $\begin{array}{l}\text { Heating } \\
\left.\qquad \frac{W}{g}\right] \\
\text { Cycle } 2\end{array}$ & $\begin{array}{c}\text { Heating } \\
{\left[\frac{W}{g}\right]} \\
\text { Cycle } 3\end{array}$ \\
\hline TEM-690 Forged & 630 & -1.39 & 8.16 & 9.07 & 9.16 \\
\hline TEM-690 Forged & 629 & -1.41 & 8.13 & 9.04 & 9.13 \\
\hline TEM-690 Forged & 628 & -1.42 & 8.05 & 8.94 & 9.03 \\
\hline TEM-690 Forged & 627 & -1.44 & 8.19 & 9.11 & 9.19 \\
\hline TEM-690 Forged & 626 & -1.45 & 8.16 & 9.07 & 9.15 \\
\hline TEM-690 PM-HIP & 625 & -1.47 & 8.20 & 9.12 & 9.21 \\
\hline TEM-690 PM-HIP & 624 & -1.48 & 8.09 & 8.99 & 9.08 \\
\hline TEM-690 PM-HIP & 623 & -1.50 & 8.06 & 8.96 & 9.05 \\
\hline TEM-690 PM-HIP & 622 & -1.51 & 8.16 & 9.07 & 9.16 \\
\hline TEM-690 PM-HIP & 621 & -1.53 & 8.14 & 9.05 & 9.13 \\
\hline TEM-690 PM-HIP & 620 & -1.54 & 8.13 & 9.04 & 9.12 \\
\hline TEM-625 Forged & 619 & -1.56 & 8.62 & 9.58 & 9.67 \\
\hline TEM-625 Forged & 618 & -1.57 & 8.67 & 9.64 & 9.73 \\
\hline TEM-625 Forged & 617 & -1.59 & 8.61 & 9.57 & 9.66 \\
\hline TEM-625 Forged & 616 & -1.60 & 8.73 & 9.71 & 9.80 \\
\hline TEM-625 Forged & 615 & -1.62 & 8.69 & 9.66 & 9.76 \\
\hline TEM-625 Forged & 614 & -1.63 & 8.74 & 9.72 & 9.81 \\
\hline TEM-625 PM-HIP & 613 & -1.65 & 8.63 & 9.59 & 9.68 \\
\hline TEM-625 PM-HIP & 612 & -1.66 & 8.55 & 9.51 & 9.60 \\
\hline TEM-625 PM-HIP & 611 & -1.68 & 8.63 & 9.60 & 9.69 \\
\hline TEM-625 PM-HIP & 610 & -1.69 & 8.53 & 9.49 & 9.58 \\
\hline TEM-625 PM-HIP & 609 & -1.71 & 8.63 & 9.59 & 9.68 \\
\hline TEM-625 PM-HIP & 608 & -1.72 & 8.52 & 9.47 & 9.56 \\
\hline CT-690 PM-HIP & 607 & -3.22 & 8.30 & 9.22 & 9.31 \\
\hline CT-690 PM-HIP & 606 & -3.22 & 8.32 & 9.25 & 9.33 \\
\hline RT-91 Cast & 605 & -8.89 & 7.70 & 8.56 & 8.64 \\
\hline RT-625 Forged & 604 & -16.51 & 8.11 & 9.02 & 9.11 \\
\hline RT-625 Forged & 603 & -24.13 & 7.66 & 8.52 & 8.60 \\
\hline RT-690 Forged & 602 & -31.75 & 6.69 & 7.44 & 7.51 \\
\hline RT-690 Forged & 601 & -39.37 & 5.82 & 6.47 & 6.53 \\
\hline
\end{tabular}




\subsection{Flux and Fluence Values}

Table 5 displays the cycles and irradiation times for the 3 DPA capsules. Tables 12-14 give the flux (total neutron flux) and fluence data for each specimen throughout the 4 rodlets that were irradiated in Cycles 164B, 166A, and 166B. The fluence data was gathered by integrating the flux values over their respective cycle times, adding the fluence values from each cycle to provide the total neutron fluence data.

Table 12. Position A-6 specimen neutron flux and fluence values of the BSU-8242-300 ${ }^{\circ} \mathrm{C} / 3$ DPA capsule analyzed at a Center Lobe Power of 19.7/21.9/22.1 MW per the three cycles.

\begin{tabular}{|c|c|c|c|c|c|c|}
\hline \multicolumn{7}{|c|}{ A-6 Position [300C 3DPA] } \\
\hline Specimen/Material & $\begin{array}{c}\text { Specimen } \\
\text { Number }\end{array}$ & $\begin{array}{l}\text { Distance } \\
\text { from Core } \\
\text { Midplane } \\
\quad(\mathrm{cm})\end{array}$ & $\begin{array}{l}\text { Neutron } \\
\text { Flux } \\
{\left[\frac{n}{\mathrm{~cm}^{2} \cdot \mathrm{s}}\right]} \\
\text { Cycle } 1\end{array}$ & $\begin{array}{l}\text { Neutron } \\
\text { Flux } \\
{\left[\frac{n}{\mathrm{~cm}^{2} \cdot \mathrm{s}}\right]} \\
\text { Cycle } 2\end{array}$ & $\begin{array}{c}\text { Neutron } \\
\text { Flux } \\
{\left[\frac{n}{\mathrm{~cm}^{2} \cdot \mathrm{s}}\right]} \\
\text { Cycle } 3\end{array}$ & $\begin{array}{l}\text { Neutron } \\
\text { Fluence } \\
{\left[\frac{n}{\mathrm{~cm}^{2}}\right]} \\
\text { Total }\end{array}$ \\
\hline RT-690 PM-HIP & 345 & 31.75 & $6.57 \mathrm{E}+14$ & $7.31 \mathrm{E}+14$ & $7.37 \mathrm{E}+14$ & $1.15 \mathrm{E}+22$ \\
\hline RT-690 PM-HIP & 344 & 24.13 & $7.44 \mathrm{E}+14$ & $8.27 \mathrm{E}+14$ & $8.35 \mathrm{E}+14$ & $1.30 \mathrm{E}+22$ \\
\hline RT-625 PM-HIP & 343 & 16.51 & $7.95 \mathrm{E}+14$ & $8.84 \mathrm{E}+14$ & $8.92 \mathrm{E}+14$ & $1.39 \mathrm{E}+22$ \\
\hline RT-625 PM-HIP & 342 & 8.89 & $8.33 \mathrm{E}+14$ & $9.26 \mathrm{E}+14$ & $9.35 \mathrm{E}+14$ & $1.46 \mathrm{E}+22$ \\
\hline CT-625 PM-HIP & 341 & 3.22 & $8.54 \mathrm{E}+14$ & $9.49 \mathrm{E}+14$ & $9.58 \mathrm{E}+14$ & $1.49 \mathrm{E}+22$ \\
\hline CT-625 PM-HIP & 340 & 3.22 & $8.55 \mathrm{E}+14$ & $9.51 \mathrm{E}+14$ & $9.60 \mathrm{E}+14$ & $1.49 \mathrm{E}+22$ \\
\hline TEM-USU-300-3-20 & 339 & 1.39 & $8.56 \mathrm{E}+14$ & $9.52 \mathrm{E}+14$ & $9.61 \mathrm{E}+14$ & $1.50 \mathrm{E}+22$ \\
\hline TEM-USU-300-3-20 & 338 & 1.37 & $8.65 \mathrm{E}+14$ & $9.62 \mathrm{E}+14$ & $9.71 \mathrm{E}+14$ & $1.51 \mathrm{E}+22$ \\
\hline TEM-USU-300-3-20 & 337 & 1.36 & $8.70 \mathrm{E}+14$ & $9.67 \mathrm{E}+14$ & $9.76 \mathrm{E}+14$ & $1.52 \mathrm{E}+22$ \\
\hline TEM-USU-300-3-28 & 336 & 1.34 & $8.68 \mathrm{E}+14$ & $9.65 \mathrm{E}+14$ & $9.74 \mathrm{E}+14$ & $1.52 \mathrm{E}+22$ \\
\hline TEM-USU-300-3-28 & 335 & 1.33 & $8.57 \mathrm{E}+14$ & $9.53 \mathrm{E}+14$ & $9.62 \mathrm{E}+14$ & $1.50 \mathrm{E}+22$ \\
\hline TEM-USU-300-3-28 & 334 & 1.31 & $8.58 \mathrm{E}+14$ & $9.54 \mathrm{E}+14$ & $9.63 \mathrm{E}+14$ & $1.50 \mathrm{E}+22$ \\
\hline TEM-USU-300-3-36 & 333 & 1.30 & $8.51 \mathrm{E}+14$ & $9.46 \mathrm{E}+14$ & $9.55 \mathrm{E}+14$ & $1.49 \mathrm{E}+22$ \\
\hline TEM-USU-300-3-36 & 332 & 1.28 & $8.41 \mathrm{E}+14$ & $9.35 \mathrm{E}+14$ & $9.44 \mathrm{E}+14$ & $1.47 \mathrm{E}+22$ \\
\hline TEM-USU-300-3-36 & 331 & 1.27 & $8.41 \mathrm{E}+14$ & $9.35 \mathrm{E}+14$ & $9.44 \mathrm{E}+14$ & $1.47 \mathrm{E}+22$ \\
\hline TEM-690 Forged & 330 & -1.38 & $8.50 \mathrm{E}+14$ & $9.45 \mathrm{E}+14$ & $9.54 \mathrm{E}+14$ & $1.48 \mathrm{E}+22$ \\
\hline TEM-690 Forged & 329 & -1.39 & $8.65 \mathrm{E}+14$ & $9.62 \mathrm{E}+14$ & $9.71 \mathrm{E}+14$ & $1.51 \mathrm{E}+22$ \\
\hline TEM-690 Forged & 328 & -1.41 & $8.75 \mathrm{E}+14$ & $9.73 \mathrm{E}+14$ & $9.82 \mathrm{E}+14$ & $1.53 \mathrm{E}+22$ \\
\hline TEM-690 Forged & 327 & -1.42 & $8.55 \mathrm{E}+14$ & $9.51 \mathrm{E}+14$ & $9.60 \mathrm{E}+14$ & $1.49 \mathrm{E}+22$ \\
\hline TEM-690 Forged & 326 & -1.44 & $8.61 \mathrm{E}+14$ & $9.58 \mathrm{E}+14$ & $9.67 \mathrm{E}+14$ & $1.51 \mathrm{E}+22$ \\
\hline TEM-690 Forged & 325 & -1.45 & $8.65 \mathrm{E}+14$ & $9.61 \mathrm{E}+14$ & $9.70 \mathrm{E}+14$ & $1.51 \mathrm{E}+22$ \\
\hline TEM-625 Forged & 324 & -1.47 & $8.63 \mathrm{E}+14$ & $9.59 \mathrm{E}+14$ & $9.68 \mathrm{E}+14$ & $1.51 \mathrm{E}+22$ \\
\hline TEM-625 Forged & 323 & -1.48 & $8.41 \mathrm{E}+14$ & $9.35 \mathrm{E}+14$ & $9.44 \mathrm{E}+14$ & $1.47 \mathrm{E}+22$ \\
\hline TEM-625 Forged & 322 & -1.50 & $8.57 \mathrm{E}+14$ & $9.53 \mathrm{E}+14$ & $9.62 \mathrm{E}+14$ & $1.50 \mathrm{E}+22$ \\
\hline TEM-625 Forged & 321 & -1.51 & $8.51 \mathrm{E}+14$ & $9.46 \mathrm{E}+14$ & $9.55 \mathrm{E}+14$ & $1.49 \mathrm{E}+22$ \\
\hline TEM-625 Forged & 320 & -1.53 & $8.48 \mathrm{E}+14$ & $9.43 \mathrm{E}+14$ & $9.52 \mathrm{E}+14$ & $1.48 \mathrm{E}+22$ \\
\hline TEM-625 Forged & 319 & -1.54 & $8.56 \mathrm{E}+14$ & $9.52 \mathrm{E}+14$ & $9.61 \mathrm{E}+14$ & $1.50 \mathrm{E}+22$ \\
\hline
\end{tabular}


BSU-8242 3 DPA As-Run Physics Analysis

\begin{tabular}{|l|c|c|c|c|c|c|}
\hline \multicolumn{7}{|c|}{ A-6 Position [300C 3DPA] } \\
\hline Specimen/Material & $\begin{array}{c}\text { Specimen } \\
\text { Number }\end{array}$ & $\begin{array}{c}\text { Distance } \\
\text { from Core } \\
\text { Midplane } \\
(\mathrm{cm})\end{array}$ & $\begin{array}{c}\text { Neutron } \\
\text { Flux } \\
{\left[\frac{n}{\mathrm{~cm}^{2} \cdot s}\right]} \\
\text { Cycle } 1\end{array}$ & $\begin{array}{c}\text { Neutron } \\
\text { Flux } \\
{\left[\frac{n}{\mathrm{~cm}^{2} \cdot s}\right]} \\
\text { Cycle } 2\end{array}$ & $\begin{array}{c}\text { Neutron } \\
\text { Flux } \\
{\left[\frac{n}{\mathrm{~cm}^{2} \cdot s}\right]} \\
\text { Cycle } 3\end{array}$ & $\begin{array}{c}\text { Neutron } \\
\text { Fluence } \\
{\left[\frac{n}{\mathrm{~cm}^{2}}\right]} \\
\text { Total }\end{array}$ \\
\hline TEM-690 PM-HIP & 318 & -1.56 & $8.41 \mathrm{E}+14$ & $9.35 \mathrm{E}+14$ & $9.43 \mathrm{E}+14$ & $1.47 \mathrm{E}+22$ \\
\hline TEM-690 PM-HIP & 317 & -1.57 & $8.43 \mathrm{E}+14$ & $9.37 \mathrm{E}+14$ & $9.46 \mathrm{E}+14$ & $1.47 \mathrm{E}+22$ \\
\hline TEM-690 PM-HIP & 316 & -1.59 & $8.56 \mathrm{E}+14$ & $9.52 \mathrm{E}+14$ & $9.61 \mathrm{E}+14$ & $1.50 \mathrm{E}+22$ \\
\hline TEM-690 PM-HIP & 315 & -1.60 & $8.53 \mathrm{E}+14$ & $9.48 \mathrm{E}+14$ & $9.57 \mathrm{E}+14$ & $1.49 \mathrm{E}+22$ \\
\hline TEM-690 PM-HIP & 314 & -1.62 & $8.51 \mathrm{E}+14$ & $9.46 \mathrm{E}+14$ & $9.55 \mathrm{E}+14$ & $1.49 \mathrm{E}+22$ \\
\hline TEM-690 PM-HIP & 313 & -1.63 & $8.54 \mathrm{E}+14$ & $9.49 \mathrm{E}+14$ & $9.58 \mathrm{E}+14$ & $1.49 \mathrm{E}+22$ \\
\hline TEM-625 PM-HIP & 312 & -1.65 & $8.69 \mathrm{E}+14$ & $9.66 \mathrm{E}+14$ & $9.75 \mathrm{E}+14$ & $1.52 \mathrm{E}+22$ \\
\hline TEM-625 PM-HIP & 311 & -1.66 & $8.69 \mathrm{E}+14$ & $9.66 \mathrm{E}+14$ & $9.75 \mathrm{E}+14$ & $1.52 \mathrm{E}+22$ \\
\hline TEM-625 PM-HIP & 310 & -1.68 & $8.71 \mathrm{E}+14$ & $9.69 \mathrm{E}+14$ & $9.78 \mathrm{E}+14$ & $1.52 \mathrm{E}+22$ \\
\hline TEM-625 PM-HIP & 309 & -1.69 & $8.75 \mathrm{E}+14$ & $9.73 \mathrm{E}+14$ & $9.82 \mathrm{E}+14$ & $1.53 \mathrm{E}+22$ \\
\hline TEM-625 PM-HIP & 308 & -1.71 & $8.85 \mathrm{E}+14$ & $9.83 \mathrm{E}+14$ & $9.93 \mathrm{E}+14$ & $1.55 \mathrm{E}+22$ \\
\hline TEM-625 PM-HIP & 307 & -1.72 & $8.67 \mathrm{E}+14$ & $9.63 \mathrm{E}+14$ & $9.72 \mathrm{E}+14$ & $1.51 \mathrm{E}+22$ \\
\hline CT-625 Cast & 306 & -3.22 & $8.56 \mathrm{E}+14$ & $9.51 \mathrm{E}+14$ & $9.60 \mathrm{E}+14$ & $1.50 \mathrm{E}+22$ \\
\hline CT-625 Cast & 305 & -3.22 & $8.56 \mathrm{E}+14$ & $9.51 \mathrm{E}+14$ & $9.60 \mathrm{E}+14$ & $1.50 \mathrm{E}+22$ \\
\hline RT-625 Forged & 304 & -8.89 & $8.40 \mathrm{E}+14$ & $9.34 \mathrm{E}+14$ & $9.43 \mathrm{E}+14$ & $1.47 \mathrm{E}+22$ \\
\hline RT-625 Forged & 303 & -16.51 & $8.15 \mathrm{E}+14$ & $9.06 \mathrm{E}+14$ & $9.14 \mathrm{E}+14$ & $1.42 \mathrm{E}+22$ \\
\hline RT-690 Forged & 302 & -24.13 & $7.77 \mathrm{E}+14$ & $8.64 \mathrm{E}+14$ & $8.72 \mathrm{E}+14$ & $1.36 \mathrm{E}+22$ \\
\hline RT-690 Forged & 301 & -31.75 & $7.07 \mathrm{E}+14$ & $7.86 \mathrm{E}+14$ & $7.93 \mathrm{E}+14$ & $1.24 \mathrm{E}+22$ \\
\hline
\end{tabular}

Table 13. Position A-7 specimen neutron flux and fluence values of the BSU-8242-400 ${ }^{\circ} \mathrm{C} / 3$ DPA (Y) capsule analyzed at a Center Lobe Power of 19.7/21.9/22.1 MW per the three cycles.

\begin{tabular}{|l|c|c|c|c|c|c|}
\hline \multicolumn{7}{|c|}{ A-7 Position [400C 3DPA (Y)] } \\
\hline Specimen/Material & $\begin{array}{c}\text { Specimen } \\
\text { Number }\end{array}$ & $\begin{array}{c}\text { Distance } \\
\text { from Core } \\
\text { Midplane } \\
(\mathrm{cm})\end{array}$ & $\begin{array}{c}\text { Neutron } \\
\text { Flux } \\
{\left[\frac{n}{\mathrm{~cm}^{2} \cdot \mathrm{s}}\right]} \\
\text { Cycle 1 }\end{array}$ & $\begin{array}{c}\text { Neutron } \\
\text { Flux } \\
{\left[\frac{n}{\mathrm{~cm}^{2} \cdot \mathrm{s}}\right]} \\
\text { Cycle 2 }\end{array}$ & $\begin{array}{c}\text { Neutron } \\
\text { Flux } \\
{\left[\frac{n}{\mathrm{~cm}^{2} \cdot \mathrm{s}}\right]} \\
\text { Cycle 3 }\end{array}$ & $\begin{array}{c}\text { Neutron } \\
\text { Fluence } \\
{\left[\frac{n}{\mathrm{~cm}^{2}}\right]} \\
\text { Total }\end{array}$ \\
\hline RT-304L Forged & 723 & 39.37 & $5.39 \mathrm{E}+14$ & $5.99 \mathrm{E}+14$ & $6.05 \mathrm{E}+14$ & $9.42 \mathrm{E}+21$ \\
\hline RT-304L Forged & 722 & 31.75 & $6.38 \mathrm{E}+14$ & $7.09 \mathrm{E}+14$ & $7.16 \mathrm{E}+14$ & $1.11 \mathrm{E}+22$ \\
\hline RT-91 PM-HIP & 721 & 24.13 & $7.21 \mathrm{E}+14$ & $8.02 \mathrm{E}+14$ & $8.09 \mathrm{E}+14$ & $1.26 \mathrm{E}+22$ \\
\hline RT-316L PM-HIP & 720 & 16.51 & $7.73 \mathrm{E}+14$ & $8.59 \mathrm{E}+14$ & $8.67 \mathrm{E}+14$ & $1.35 \mathrm{E}+22$ \\
\hline RT-316L PM-HIP & 719 & 8.89 & $8.07 \mathrm{E}+14$ & $8.97 \mathrm{E}+14$ & $9.06 \mathrm{E}+14$ & $1.41 \mathrm{E}+22$ \\
\hline CT-690 Forged & 718 & 3.22 & $8.24 \mathrm{E}+14$ & $9.16 \mathrm{E}+14$ & $9.24 \mathrm{E}+14$ & $1.44 \mathrm{E}+22$ \\
\hline CT-316 Cast & 717 & 3.22 & $8.24 \mathrm{E}+14$ & $9.16 \mathrm{E}+14$ & $9.25 \mathrm{E}+14$ & $1.44 \mathrm{E}+22$ \\
\hline TEM-USU-400-3-36 & 716 & 1.57 & $8.20 \mathrm{E}+14$ & $9.12 \mathrm{E}+14$ & $9.20 \mathrm{E}+14$ & $1.43 \mathrm{E}+22$ \\
\hline
\end{tabular}


ENGINEERING CALCULATIONS AND ANALYSIS

BSU-8242 3 DPA As-Run Physics Analysis
ECAR- 4951, Rev. 1 Page 36 of 104

\begin{tabular}{|c|c|c|c|c|c|c|}
\hline \multicolumn{7}{|c|}{ A-7 Position [400C 3DPA (Y)] } \\
\hline Specimen/Material & $\begin{array}{l}\text { Specimen } \\
\text { Number }\end{array}$ & $\begin{array}{l}\text { Distance } \\
\text { from Core } \\
\text { Midplane } \\
(\mathrm{cm})\end{array}$ & $\begin{array}{l}\text { Neutron } \\
\text { Flux } \\
{\left[\frac{n}{\mathrm{~cm}^{2} \cdot s}\right]} \\
\text { Cycle } 1\end{array}$ & $\begin{array}{l}\text { Neutron } \\
\text { Flux } \\
{\left[\frac{n}{\mathrm{~cm}^{2} \cdot \mathrm{s}}\right]} \\
\text { Cycle } 2\end{array}$ & $\begin{array}{l}\text { Neutron } \\
\text { Flux } \\
{\left[\frac{n}{\mathrm{~cm}^{2} \cdot \mathrm{s}}\right]} \\
\text { Cycle } 3\end{array}$ & $\begin{array}{l}\text { Neutron } \\
\text { Fluence } \\
{\left[\frac{n}{c m^{2}}\right]} \\
\text { Total }\end{array}$ \\
\hline TEM-USU-400-3-36 & 715 & 1.54 & $8.31 \mathrm{E}+14$ & $9.24 \mathrm{E}+14$ & $9.33 \mathrm{E}+14$ & $1.45 \mathrm{E}+22$ \\
\hline TEM-USU-400-3-36 & 714 & 1.51 & $8.29 E+14$ & $9.21 \mathrm{E}+14$ & $9.30 \mathrm{E}+14$ & $1.45 \mathrm{E}+22$ \\
\hline TEM-USU-400-3-28 & 713 & 1.48 & $8.12 \mathrm{E}+14$ & $9.03 E+14$ & $9.11 \mathrm{E}+14$ & $1.42 \mathrm{E}+22$ \\
\hline TEM-USU-400-3-28 & 712 & 1.45 & $8.03 \mathrm{E}+14$ & $8.93 \mathrm{E}+14$ & $9.02 \mathrm{E}+14$ & $1.40 \mathrm{E}+22$ \\
\hline TEM-USU-400-3-28 & 711 & 1.42 & $8.05 E+14$ & $8.95 E+14$ & $9.04 \mathrm{E}+14$ & $1.41 \mathrm{E}+22$ \\
\hline TEM-USU-400-3-20 & 710 & 1.39 & $8.01 \mathrm{E}+14$ & $8.91 \mathrm{E}+14$ & $8.99 \mathrm{E}+14$ & $1.40 \mathrm{E}+22$ \\
\hline TEM-USU-400-3-20 & 709 & 1.36 & $8.03 E+14$ & $8.92 \mathrm{E}+14$ & $9.01 \mathrm{E}+14$ & $1.40 \mathrm{E}+22$ \\
\hline TEM-USU-400-3-20 & 708 & 1.33 & $8.06 \mathrm{E}+14$ & $8.96 \mathrm{E}+14$ & $9.04 \mathrm{E}+14$ & $1.41 \mathrm{E}+22$ \\
\hline CT-316 PM-HIP & 707 & -3.22 & $8.30 \mathrm{E}+14$ & $9.22 \mathrm{E}+14$ & $9.31 \mathrm{E}+14$ & $1.45 \mathrm{E}+22$ \\
\hline CT-316 PM-HIP & 706 & -3.22 & $8.30 \mathrm{E}+14$ & $9.22 \mathrm{E}+14$ & $9.31 \mathrm{E}+14$ & $1.45 \mathrm{E}+22$ \\
\hline RT-316L Forged & 705 & -8.89 & $8.13 E+14$ & $9.04 \mathrm{E}+14$ & $9.13 \mathrm{E}+14$ & $1.42 \mathrm{E}+22$ \\
\hline RT-316L Forged & 704 & -16.51 & $7.89 \mathrm{E}+14$ & $8.77 \mathrm{E}+14$ & $8.85 \mathrm{E}+14$ & $1.38 \mathrm{E}+22$ \\
\hline RT-91 Cast & 703 & -24.13 & $7.52 \mathrm{E}+14$ & $8.36 \mathrm{E}+14$ & $8.44 \mathrm{E}+14$ & $1.31 \mathrm{E}+22$ \\
\hline RT-304L Forged & 702 & -31.75 & $6.82 \mathrm{E}+14$ & $7.58 \mathrm{E}+14$ & $7.65 \mathrm{E}+14$ & $1.19 \mathrm{E}+22$ \\
\hline RT-304L Forged & 701 & -39.37 & $5.97 \mathrm{E}+14$ & $6.63 \mathrm{E}+14$ & $6.70 \mathrm{E}+14$ & $1.04 \mathrm{E}+22$ \\
\hline
\end{tabular}

Table 14. Position A-8 specimen neutron flux and fluence values of the BSU-8242-400 ${ }^{\circ} \mathrm{C} / 3$ DPA (X) capsule analyzed at a Center Lobe Power of 19.7/21.9/22.1 MW per the three cycles.

\begin{tabular}{|l|c|c|c|c|c|c|}
\hline \multicolumn{7}{|c|}{ A-8 Position [400C 3DPA (X)] } \\
\hline Specimen/Material & $\begin{array}{c}\text { Specimen } \\
\text { Number }\end{array}$ & $\begin{array}{c}\text { Distance } \\
\text { from Core } \\
\text { Midplane } \\
(\mathrm{cm})\end{array}$ & $\begin{array}{c}\text { Neutron } \\
\text { Flux } \\
{\left[\frac{n}{\mathrm{~cm}^{2} \cdot \mathrm{s}}\right]} \\
\text { Cycle } 1\end{array}$ & $\begin{array}{c}\text { Neutron } \\
\text { Flux } \\
{\left[\frac{n}{\mathrm{~cm}^{2} \cdot s}\right]} \\
\text { Cycle 2 }\end{array}$ & $\begin{array}{c}\text { Neutron } \\
\text { Flux } \\
{\left[\frac{n}{\mathrm{~cm}^{2} \cdot s}\right]} \\
\text { Cycle } 3\end{array}$ & $\left.\begin{array}{c}\text { Neutron } \\
\text { Fluence } \\
{\left[\frac{n}{\mathrm{~cm}^{2}}\right.}\end{array}\right]$ \\
\hline Total \\
\hline RT-690 PM-HIP & 674 & 39.37 & $5.27 \mathrm{E}+14$ & $5.86 \mathrm{E}+14$ & $5.92 \mathrm{E}+14$ & $9.21 \mathrm{E}+21$ \\
\hline RT-625 PM-HIP & 673 & 31.75 & $6.24 \mathrm{E}+14$ & $6.93 \mathrm{E}+14$ & $7.00 \mathrm{E}+14$ & $1.09 \mathrm{E}+22$ \\
\hline RT-625 PM-HIP & 672 & 24.13 & $7.01 \mathrm{E}+14$ & $7.79 \mathrm{E}+14$ & $7.86 \mathrm{E}+14$ & $1.22 \mathrm{E}+22$ \\
\hline RT-91 PM-HIP & 671 & 16.51 & $7.51 \mathrm{E}+14$ & $8.35 \mathrm{E}+14$ & $8.43 \mathrm{E}+14$ & $1.31 \mathrm{E}+22$ \\
\hline CT-625 PM-HIP & 669 & 3.22 & $8.08 \mathrm{E}+14$ & $8.98 \mathrm{E}+14$ & $9.06 \mathrm{E}+14$ & $1.41 \mathrm{E}+22$ \\
\hline CT-625 Cast & 668 & 3.22 & $8.10 \mathrm{E}+14$ & $9.00 \mathrm{E}+14$ & $9.09 \mathrm{E}+14$ & $1.42 \mathrm{E}+22$ \\
\hline TEM-91 Cast & 667 & 1.70 & $8.20 \mathrm{E}+14$ & $9.11 \mathrm{E}+14$ & $9.20 \mathrm{E}+14$ & $1.43 \mathrm{E}+22$ \\
\hline TEM-91 Cast & 666 & 1.69 & $8.24 \mathrm{E}+14$ & $9.16 \mathrm{E}+14$ & $9.25 \mathrm{E}+14$ & $1.44 \mathrm{E}+22$ \\
\hline TEM-91 Cast & 665 & 1.67 & $8.17 \mathrm{E}+14$ & $9.09 \mathrm{E}+14$ & $9.17 \mathrm{E}+14$ & $1.43 \mathrm{E}+22$ \\
\hline TEM-91 Cast & 664 & 1.66 & $8.15 \mathrm{E}+14$ & $9.06 \mathrm{E}+14$ & $9.15 \mathrm{E}+14$ & $1.42 \mathrm{E}+22$ \\
\hline
\end{tabular}


ENGINEERING CALCULATIONS AND ANALYSIS

BSU-8242 3 DPA As-Run Physics Analysis
ECAR- 4951, Rev. 1

Page 37 of 104

\begin{tabular}{|c|c|c|c|c|c|c|}
\hline \multicolumn{7}{|c|}{ A-8 Position [400C 3DPA (X)] } \\
\hline Specimen/Material & $\begin{array}{c}\text { Specimen } \\
\text { Number }\end{array}$ & $\begin{array}{l}\text { Distance } \\
\text { from Core } \\
\text { Midplane } \\
(\mathrm{cm})\end{array}$ & $\begin{array}{l}\text { Neutron } \\
\text { Flux } \\
{\left[\frac{n}{\mathrm{~cm}^{2} \cdot \mathrm{s}}\right]} \\
\text { Cycle } 1\end{array}$ & $\begin{array}{l}\text { Neutron } \\
\text { Flux } \\
{\left[\frac{n}{\mathrm{~cm}^{2} \cdot \mathrm{s}}\right]} \\
\text { Cycle } 2\end{array}$ & $\begin{array}{l}\text { Neutron } \\
\text { Flux } \\
{\left[\frac{n}{\mathrm{~cm}^{2} \cdot \mathrm{s}}\right]} \\
\text { Cycle } 3\end{array}$ & $\begin{array}{l}\text { Neutron } \\
\text { Fluence } \\
{\left[\frac{n}{\mathrm{~cm}^{2}}\right]} \\
\text { Total }\end{array}$ \\
\hline TEM-91 Cast & 663 & 1.64 & $8.09 \mathrm{E}+14$ & $8.99 \mathrm{E}+14$ & $9.07 \mathrm{E}+14$ & $1.41 \mathrm{E}+22$ \\
\hline TEM-91 Cast & 662 & 1.63 & $8.19 \mathrm{E}+14$ & $9.10 \mathrm{E}+14$ & $9.19 \mathrm{E}+14$ & $1.43 \mathrm{E}+22$ \\
\hline TEM-304L Forged & 661 & 1.61 & $8.19 \mathrm{E}+14$ & $9.10 \mathrm{E}+14$ & $9.19 \mathrm{E}+14$ & $1.43 \mathrm{E}+22$ \\
\hline TEM-304L Forged & 660 & 1.60 & $8.22 \mathrm{E}+14$ & $9.14 \mathrm{E}+14$ & $9.23 \mathrm{E}+14$ & $1.44 \mathrm{E}+22$ \\
\hline TEM-304L Forged & 659 & 1.58 & $8.36 \mathrm{E}+14$ & $9.30 \mathrm{E}+14$ & $9.38 \mathrm{E}+14$ & $1.46 \mathrm{E}+22$ \\
\hline TEM-304L Forged & 658 & 1.57 & $8.30 \mathrm{E}+14$ & $9.22 \mathrm{E}+14$ & $9.31 \mathrm{E}+14$ & $1.45 \mathrm{E}+22$ \\
\hline TEM-304L Forged & 657 & 1.55 & $8.34 \mathrm{E}+14$ & $9.27 \mathrm{E}+14$ & $9.36 \mathrm{E}+14$ & $1.46 \mathrm{E}+22$ \\
\hline TEM-304L Forged & 656 & 1.54 & $8.25 \mathrm{E}+14$ & $9.18 \mathrm{E}+14$ & $9.26 \mathrm{E}+14$ & $1.44 \mathrm{E}+22$ \\
\hline TEM-304L Forged & 655 & 1.52 & $8.36 \mathrm{E}+14$ & $9.30 \mathrm{E}+14$ & $9.38 \mathrm{E}+14$ & $1.46 \mathrm{E}+22$ \\
\hline TEM-304L Forged & 654 & 1.51 & $8.28 \mathrm{E}+14$ & $9.20 \mathrm{E}+14$ & $9.29 \mathrm{E}+14$ & $1.45 \mathrm{E}+22$ \\
\hline TEM-304L Forged & 653 & 1.49 & $8.26 \mathrm{E}+14$ & $9.18 \mathrm{E}+14$ & $9.27 \mathrm{E}+14$ & $1.44 \mathrm{E}+22$ \\
\hline TEM-304L Forged & 652 & 1.48 & $8.24 \mathrm{E}+14$ & $9.16 \mathrm{E}+14$ & $9.25 \mathrm{E}+14$ & $1.44 \mathrm{E}+22$ \\
\hline TEM-304L Forged & 651 & 1.46 & $8.30 \mathrm{E}+14$ & $9.22 \mathrm{E}+14$ & $9.31 \mathrm{E}+14$ & $1.45 \mathrm{E}+22$ \\
\hline TEM-304L Forged & 650 & 1.45 & $8.31 \mathrm{E}+14$ & $9.24 \mathrm{E}+14$ & $9.33 \mathrm{E}+14$ & $1.45 \mathrm{E}+22$ \\
\hline TEM-316L PM-HIP & 649 & 1.43 & $8.31 \mathrm{E}+14$ & $9.24 \mathrm{E}+14$ & $9.33 \mathrm{E}+14$ & $1.45 \mathrm{E}+22$ \\
\hline TEM-316L PM-HIP & 648 & 1.42 & $8.33 \mathrm{E}+14$ & $9.26 \mathrm{E}+14$ & $9.35 \mathrm{E}+14$ & $1.46 \mathrm{E}+22$ \\
\hline TEM-316L PM-HIP & 647 & 1.40 & $8.36 \mathrm{E}+14$ & $9.30 \mathrm{E}+14$ & $9.38 \mathrm{E}+14$ & $1.46 \mathrm{E}+22$ \\
\hline TEM-316L PM-HIP & 646 & 1.39 & $8.31 \mathrm{E}+14$ & $9.24 \mathrm{E}+14$ & $9.33 \mathrm{E}+14$ & $1.45 \mathrm{E}+22$ \\
\hline TEM-316L PM-HIP & 645 & 1.37 & $8.34 \mathrm{E}+14$ & $9.27 \mathrm{E}+14$ & $9.35 \mathrm{E}+14$ & $1.46 \mathrm{E}+22$ \\
\hline TEM-316L PM-HIP & 644 & 1.36 & $8.32 \mathrm{E}+14$ & $9.25 \mathrm{E}+14$ & $9.33 \mathrm{E}+14$ & $1.45 \mathrm{E}+22$ \\
\hline TEM-316L Forged & 643 & 1.34 & $8.36 \mathrm{E}+14$ & $9.29 \mathrm{E}+14$ & $9.38 \mathrm{E}+14$ & $1.46 \mathrm{E}+22$ \\
\hline TEM-316L Forged & 642 & 1.33 & $8.37 \mathrm{E}+14$ & $9.30 \mathrm{E}+14$ & $9.39 \mathrm{E}+14$ & $1.46 \mathrm{E}+22$ \\
\hline TEM-316L Forged & 641 & 1.31 & $8.33 \mathrm{E}+14$ & $9.26 \mathrm{E}+14$ & $9.35 \mathrm{E}+14$ & $1.46 \mathrm{E}+22$ \\
\hline TEM-316L Forged & 640 & 1.30 & $8.23 \mathrm{E}+14$ & $9.15 \mathrm{E}+14$ & $9.24 \mathrm{E}+14$ & $1.44 \mathrm{E}+22$ \\
\hline TEM-316L Forged & 639 & 1.28 & $8.15 \mathrm{E}+14$ & $9.06 \mathrm{E}+14$ & $9.14 \mathrm{E}+14$ & $1.42 \mathrm{E}+22$ \\
\hline TEM-316L Forged & 638 & 1.27 & $8.13 \mathrm{E}+14$ & $9.03 \mathrm{E}+14$ & $9.12 \mathrm{E}+14$ & $1.42 \mathrm{E}+22$ \\
\hline TEM-91 PM-HIP & 637 & -1.29 & $8.11 \mathrm{E}+14$ & $9.01 \mathrm{E}+14$ & $9.10 \mathrm{E}+14$ & $1.42 \mathrm{E}+22$ \\
\hline TEM-91 PM-HIP & 636 & -1.30 & $8.23 \mathrm{E}+14$ & $9.15 \mathrm{E}+14$ & $9.23 \mathrm{E}+14$ & $1.44 \mathrm{E}+22$ \\
\hline TEM-91 PM-HIP & 635 & -1.32 & $8.06 \mathrm{E}+14$ & $8.96 \mathrm{E}+14$ & $9.05 \mathrm{E}+14$ & $1.41 \mathrm{E}+22$ \\
\hline TEM-91 PM-HIP & 634 & -1.33 & $7.99 \mathrm{E}+14$ & $8.89 E+14$ & $8.97 \mathrm{E}+14$ & $1.40 \mathrm{E}+22$ \\
\hline TEM-91 PM-HIP & 633 & -1.35 & $7.93 \mathrm{E}+14$ & $8.82 \mathrm{E}+14$ & $8.90 \mathrm{E}+14$ & $1.39 \mathrm{E}+22$ \\
\hline TEM-91 PM-HIP & 632 & -1.36 & $8.08 \mathrm{E}+14$ & $8.98 \mathrm{E}+14$ & $9.06 \mathrm{E}+14$ & $1.41 \mathrm{E}+22$ \\
\hline TEM-690 Forged & 631 & -1.38 & $8.13 E+14$ & $9.04 \mathrm{E}+14$ & $9.13 \mathrm{E}+14$ & $1.42 \mathrm{E}+22$ \\
\hline
\end{tabular}


ENGINEERING CALCULATIONS AND ANALYSIS

BSU-8242 3 DPA As-Run Physics Analysis
ECAR- 4951, Rev. 1

Page 38 of 104

\begin{tabular}{|c|c|c|c|c|c|c|}
\hline \multicolumn{7}{|c|}{ A-8 Position [400C 3DPA (X)] } \\
\hline Specimen/Material & $\begin{array}{l}\text { Specimen } \\
\text { Number }\end{array}$ & $\begin{array}{l}\text { Distance } \\
\text { from Core } \\
\text { Midplane } \\
(\mathrm{cm})\end{array}$ & $\begin{array}{l}\text { Neutron } \\
\text { Flux } \\
{\left[\frac{n}{\mathrm{~cm}^{2} \cdot \mathrm{s}}\right]} \\
\text { Cycle } 1\end{array}$ & $\begin{array}{c}\text { Neutron } \\
\text { Flux } \\
{\left[\frac{n}{\mathrm{~cm}^{2} \cdot \mathrm{s}}\right]} \\
\text { Cycle } 2\end{array}$ & $\begin{array}{c}\text { Neutron } \\
\text { Flux } \\
{\left[\frac{n}{\mathrm{~cm}^{2} \cdot \mathrm{s}}\right]} \\
\text { Cycle } 3\end{array}$ & $\begin{array}{c}\text { Neutron } \\
\text { Fluence } \\
{\left[\frac{n}{c m^{2}}\right]} \\
\text { Total }\end{array}$ \\
\hline TEM-690 Forged & 630 & -1.39 & $8.26 \mathrm{E}+14$ & $9.18 \mathrm{E}+14$ & $9.27 \mathrm{E}+14$ & $1.44 \mathrm{E}+22$ \\
\hline TEM-690 Forged & 629 & -1.41 & $8.19 \mathrm{E}+14$ & $9.11 \mathrm{E}+14$ & $9.20 \mathrm{E}+14$ & $1.43 \mathrm{E}+22$ \\
\hline TEM-690 Forged & 628 & -1.42 & $8.26 \mathrm{E}+14$ & $9.18 \mathrm{E}+14$ & $9.27 \mathrm{E}+14$ & $1.44 \mathrm{E}+22$ \\
\hline TEM-690 Forged & 627 & -1.44 & $8.12 \mathrm{E}+14$ & $9.03 E+14$ & $9.11 \mathrm{E}+14$ & $1.42 \mathrm{E}+22$ \\
\hline TEM-690 Forged & 626 & -1.45 & $8.16 \mathrm{E}+14$ & $9.07 \mathrm{E}+14$ & $9.16 \mathrm{E}+14$ & $1.43 E+22$ \\
\hline TEM-690 PM-HIP & 625 & -1.47 & $8.23 \mathrm{E}+14$ & $9.15 \mathrm{E}+14$ & $9.23 \mathrm{E}+14$ & $1.44 \mathrm{E}+22$ \\
\hline TEM-690 PM-HIP & 624 & -1.48 & $8.14 \mathrm{E}+14$ & $9.05 \mathrm{E}+14$ & $9.14 \mathrm{E}+14$ & $1.42 \mathrm{E}+22$ \\
\hline TEM-690 PM-HIP & 623 & -1.50 & $8.11 \mathrm{E}+14$ & $9.01 \mathrm{E}+14$ & $9.10 \mathrm{E}+14$ & $1.42 \mathrm{E}+22$ \\
\hline TEM-690 PM-HIP & 622 & -1.51 & $8.17 \mathrm{E}+14$ & $9.09 \mathrm{E}+14$ & $9.17 \mathrm{E}+14$ & $1.43 E+22$ \\
\hline TEM-690 PM-HIP & 621 & -1.53 & $8.15 \mathrm{E}+14$ & $9.07 \mathrm{E}+14$ & $9.15 \mathrm{E}+14$ & $1.43 \mathrm{E}+22$ \\
\hline TEM-690 PM-HIP & 620 & -1.54 & $8.08 \mathrm{E}+14$ & $8.98 \mathrm{E}+14$ & $9.07 \mathrm{E}+14$ & $1.41 \mathrm{E}+22$ \\
\hline TEM-625 Forged & 619 & -1.56 & $8.21 \mathrm{E}+14$ & $9.13 \mathrm{E}+14$ & $9.21 \mathrm{E}+14$ & $1.43 E+22$ \\
\hline TEM-625 Forged & 618 & -1.57 & $8.25 \mathrm{E}+14$ & $9.18 \mathrm{E}+14$ & $9.26 \mathrm{E}+14$ & $1.44 \mathrm{E}+22$ \\
\hline TEM-625 Forged & 617 & -1.59 & $8.29 \mathrm{E}+14$ & $9.22 \mathrm{E}+14$ & $9.31 \mathrm{E}+14$ & $1.45 \mathrm{E}+22$ \\
\hline TEM-625 Forged & 616 & -1.60 & $8.09 \mathrm{E}+14$ & $8.99 \mathrm{E}+14$ & $9.08 \mathrm{E}+14$ & $1.41 \mathrm{E}+22$ \\
\hline TEM-625 Forged & 615 & -1.62 & $8.02 \mathrm{E}+14$ & $8.92 \mathrm{E}+14$ & $9.00 \mathrm{E}+14$ & $1.40 \mathrm{E}+22$ \\
\hline TEM-625 Forged & 614 & -1.63 & $7.93 E+14$ & $8.81 \mathrm{E}+14$ & $8.90 \mathrm{E}+14$ & $1.39 \mathrm{E}+22$ \\
\hline TEM-625 PM-HIP & 613 & -1.65 & $8.05 \mathrm{E}+14$ & $8.95 \mathrm{E}+14$ & $9.03 \mathrm{E}+14$ & $1.41 \mathrm{E}+22$ \\
\hline TEM-625 PM-HIP & 612 & -1.66 & $8.02 \mathrm{E}+14$ & $8.92 \mathrm{E}+14$ & $9.00 \mathrm{E}+14$ & $1.40 \mathrm{E}+22$ \\
\hline TEM-625 PM-HIP & 611 & -1.68 & $8.03 E+14$ & $8.93 \mathrm{E}+14$ & $9.01 \mathrm{E}+14$ & $1.40 \mathrm{E}+22$ \\
\hline TEM-625 PM-HIP & 610 & -1.69 & $8.00 \mathrm{E}+14$ & $8.90 \mathrm{E}+14$ & $8.98 \mathrm{E}+14$ & $1.40 \mathrm{E}+22$ \\
\hline TEM-625 PM-HIP & 609 & -1.71 & $8.14 \mathrm{E}+14$ & $9.05 \mathrm{E}+14$ & $9.13 \mathrm{E}+14$ & $1.42 \mathrm{E}+22$ \\
\hline TEM-625 PM-HIP & 608 & -1.72 & $8.20 \mathrm{E}+14$ & $9.12 \mathrm{E}+14$ & $9.21 \mathrm{E}+14$ & $1.43 \mathrm{E}+22$ \\
\hline CT-690 PM-HIP & 607 & -3.22 & $8.10 \mathrm{E}+14$ & $9.00 \mathrm{E}+14$ & $9.09 \mathrm{E}+14$ & $1.41 \mathrm{E}+22$ \\
\hline CT-690 PM-HIP & 606 & -3.22 & $8.10 \mathrm{E}+14$ & $9.00 \mathrm{E}+14$ & $9.09 \mathrm{E}+14$ & $1.41 \mathrm{E}+22$ \\
\hline RT-91 Cast & 605 & -8.89 & $8.03 \mathrm{E}+14$ & $8.93 \mathrm{E}+14$ & $9.01 \mathrm{E}+14$ & $1.40 \mathrm{E}+22$ \\
\hline RT-625 Forged & 604 & -16.51 & $7.69 \mathrm{E}+14$ & $8.55 \mathrm{E}+14$ & $8.63 \mathrm{E}+14$ & $1.34 \mathrm{E}+22$ \\
\hline RT-625 Forged & 603 & -24.13 & $7.30 \mathrm{E}+14$ & $8.12 \mathrm{E}+14$ & $8.19 \mathrm{E}+14$ & $1.28 \mathrm{E}+22$ \\
\hline RT-690 Forged & 602 & -31.75 & $6.68 \mathrm{E}+14$ & $7.42 \mathrm{E}+14$ & $7.50 \mathrm{E}+14$ & $1.17 \mathrm{E}+22$ \\
\hline RT-690 Forged & 601 & -39.37 & $5.85 \mathrm{E}+14$ & $6.51 \mathrm{E}+14$ & $6.57 \mathrm{E}+14$ & $1.02 \mathrm{E}+22$ \\
\hline
\end{tabular}


BSU-8242 3 DPA As-Run Physics Analysis

\subsection{Displacements Per Atom}

The following three tables (Tables 15-17) provide the DPA values for each test specimen within each of the capsules. The tally output data was scaled to the appropriate lobe powers specific to each cycle.

Table 15 . Position A-6 specimen DPA values of the BSU-8242-300 ${ }^{\circ} \mathrm{C} / 3$ DPA capsule analyzed at a Center Lobe Power of 19.7/21.9/22.1 MW per the three cycles.

\begin{tabular}{|c|c|c|c|c|c|c|}
\hline \multicolumn{6}{|c|}{ A-6 Position [300C 3DPA] } & \multirow[b]{2}{*}{$\begin{array}{l}\text { Total } \\
\text { DPA }\end{array}$} \\
\hline Specimen/Material & $\begin{array}{l}\text { Specimen } \\
\text { Number }\end{array}$ & $\begin{array}{l}\text { Distance } \\
\text { from Core } \\
\text { Midplane } \\
(\mathrm{cm})\end{array}$ & $\begin{array}{c}\text { DPA } \\
\text { Cycle } 1\end{array}$ & $\begin{array}{c}\text { DPA } \\
\text { Cycle } 2\end{array}$ & $\begin{array}{c}\text { DPA } \\
\text { Cycle } 3\end{array}$ & \\
\hline RT-690 PM-HIP & 345 & 31.75 & 1.12 & 1.21 & 1.20 & 3.53 \\
\hline RT-690 PM-HIP & 344 & 24.13 & 1.26 & 1.36 & 1.35 & 3.96 \\
\hline RT-625 PM-HIP & 343 & 16.51 & 1.34 & 1.45 & 1.44 & 4.23 \\
\hline RT-625 PM-HIP & 342 & 8.89 & 1.39 & 1.51 & 1.49 & 4.40 \\
\hline CT-625 PM-HIP & 341 & 3.22 & 1.42 & 1.54 & 1.52 & 4.47 \\
\hline CT-625 PM-HIP & 340 & 3.22 & 1.42 & 1.54 & 1.52 & 4.48 \\
\hline TEM-USU-300-3-20 & 339 & 1.39 & 1.98 & 2.14 & 2.12 & 6.24 \\
\hline TEM-USU-300-3-20 & 338 & 1.37 & 1.97 & 2.13 & 2.11 & 6.21 \\
\hline TEM-USU-300-3-20 & 337 & 1.36 & 1.98 & 2.15 & 2.13 & 6.26 \\
\hline TEM-USU-300-3-28 & 336 & 1.34 & 1.89 & 2.05 & 2.02 & 5.96 \\
\hline TEM-USU-300-3-28 & 335 & 1.33 & 1.82 & 1.98 & 1.95 & 5.75 \\
\hline TEM-USU-300-3-28 & 334 & 1.31 & 1.79 & 1.94 & 1.92 & 5.66 \\
\hline TEM-USU-300-3-36 & 333 & 1.30 & 1.70 & 1.84 & 1.82 & 5.37 \\
\hline TEM-USU-300-3-36 & 332 & 1.28 & 1.70 & 1.84 & 1.82 & 5.35 \\
\hline TEM-USU-300-3-36 & 331 & 1.27 & 1.72 & 1.87 & 1.84 & 5.43 \\
\hline TEM-690 Forged & 330 & -1.38 & 1.59 & 1.72 & 1.70 & 5.01 \\
\hline TEM-690 Forged & 329 & -1.39 & 1.46 & 1.59 & 1.57 & 4.61 \\
\hline TEM-690 Forged & 328 & -1.41 & 1.44 & 1.56 & 1.54 & 4.55 \\
\hline TEM-690 Forged & 327 & -1.42 & 1.43 & 1.55 & 1.54 & 4.52 \\
\hline TEM-690 Forged & 326 & -1.44 & 1.45 & 1.57 & 1.55 & 4.56 \\
\hline TEM-690 Forged & 325 & -1.45 & 1.43 & 1.55 & 1.54 & 4.52 \\
\hline TEM-625 Forged & 324 & -1.47 & 1.46 & 1.58 & 1.56 & 4.60 \\
\hline TEM-625 Forged & 323 & -1.48 & 1.42 & 1.54 & 1.52 & 4.47 \\
\hline TEM-625 Forged & 322 & -1.50 & 1.42 & 1.54 & 1.52 & 4.49 \\
\hline TEM-625 Forged & 321 & -1.51 & 1.41 & 1.53 & 1.51 & 4.46 \\
\hline TEM-625 Forged & 320 & -1.53 & 1.39 & 1.51 & 1.49 & 4.39 \\
\hline TEM-625 Forged & 319 & -1.54 & 1.46 & 1.59 & 1.57 & 4.62 \\
\hline TEM-690 PM-HIP & 318 & -1.56 & 1.40 & 1.52 & 1.50 & 4.42 \\
\hline TEM-690 PM-HIP & 317 & -1.57 & 1.41 & 1.53 & 1.52 & 4.46 \\
\hline
\end{tabular}


BSU-8242 3 DPA As-Run Physics Analysis

\begin{tabular}{|c|c|c|c|c|c|c|}
\hline \multicolumn{6}{|c|}{ A-6 Position [300C 3DPA] } & \multirow[b]{2}{*}{$\begin{array}{l}\text { Total } \\
\text { DPA }\end{array}$} \\
\hline Specimen/Material & $\begin{array}{c}\text { Specimen } \\
\text { Number }\end{array}$ & $\begin{array}{l}\text { Distance } \\
\text { from Core } \\
\text { Midplane } \\
(\mathrm{cm})\end{array}$ & $\begin{array}{c}\text { DPA } \\
\text { Cycle } 1\end{array}$ & $\begin{array}{c}\text { DPA } \\
\text { Cycle } 2\end{array}$ & $\begin{array}{c}\text { DPA } \\
\text { Cycle } 3\end{array}$ & \\
\hline TEM-690 PM-HIP & 316 & -1.59 & 1.37 & 1.49 & 1.47 & 4.33 \\
\hline TEM-690 PM-HIP & 315 & -1.60 & 1.39 & 1.51 & 1.49 & 4.40 \\
\hline TEM-690 PM-HIP & 314 & -1.62 & 1.42 & 1.53 & 1.52 & 4.47 \\
\hline TEM-690 PM-HIP & 313 & -1.63 & 1.42 & 1.54 & 1.52 & 4.48 \\
\hline TEM-625 PM-HIP & 312 & -1.65 & 1.45 & 1.57 & 1.55 & 4.57 \\
\hline TEM-625 PM-HIP & 311 & -1.66 & 1.45 & 1.58 & 1.56 & 4.59 \\
\hline TEM-625 PM-HIP & 310 & -1.68 & 1.44 & 1.56 & 1.55 & 4.55 \\
\hline TEM-625 PM-HIP & 309 & -1.69 & 1.42 & 1.54 & 1.52 & 4.48 \\
\hline TEM-625 PM-HIP & 308 & -1.71 & 1.46 & 1.58 & 1.56 & 4.60 \\
\hline TEM-625 PM-HIP & 307 & -1.72 & 1.40 & 1.51 & 1.49 & 4.40 \\
\hline CT-625 Cast & 306 & -3.22 & 1.42 & 1.54 & 1.52 & 4.48 \\
\hline CT-625 Cast & 305 & -3.22 & 1.42 & 1.54 & 1.52 & 4.47 \\
\hline RT-625 Forged & 304 & -8.89 & 1.41 & 1.52 & 1.51 & 4.43 \\
\hline RT-625 Forged & 303 & -16.51 & 1.37 & 1.48 & 1.47 & 4.32 \\
\hline RT-690 Forged & 302 & -24.13 & 1.31 & 1.42 & 1.40 & 4.13 \\
\hline RT-690 Forged & 301 & -31.75 & 1.20 & 1.30 & 1.29 & 3.79 \\
\hline
\end{tabular}

Table 16. Position A-7 specimen DPA values of the BSU-8242-400 ${ }^{\circ} \mathrm{C} / 3$ DPA (Y) capsule analyzed at a Center Lobe Power of 19.7/21.9/22.1 MW per the three cycles.

A-7 Position [400C 3DPA (Y)]

\begin{tabular}{|c|c|c|c|c|c|c|}
\hline Specimen/Material & $\begin{array}{c}\text { Specimen } \\
\text { Number }\end{array}$ & $\begin{array}{l}\text { Distance } \\
\text { from Core } \\
\text { Midplane } \\
(\mathrm{cm})\end{array}$ & $\begin{array}{c}\text { DPA } \\
\text { Cycle } 1\end{array}$ & $\begin{array}{c}\text { DPA } \\
\text { Cycle } 2\end{array}$ & $\begin{array}{c}\text { DPA } \\
\text { Cycle } 3\end{array}$ & $\begin{array}{l}\text { Total } \\
\text { DPA }\end{array}$ \\
\hline RT-304L Forged & 723 & 39.37 & 0.86 & 0.93 & 0.92 & 2.70 \\
\hline RT-304L Forged & 722 & 31.75 & 1.00 & 1.08 & 1.07 & 3.15 \\
\hline RT-91 PM-HIP & 721 & 24.13 & 1.08 & 1.17 & 1.15 & 3.40 \\
\hline RT-316L PM-HIP & 720 & 16.51 & 1.19 & 1.30 & 1.28 & 3.77 \\
\hline RT-316L PM-HIP & 719 & 8.89 & 1.24 & 1.34 & 1.33 & 3.91 \\
\hline CT-690 Forged & 718 & 3.22 & 1.35 & 1.46 & 1.45 & 4.26 \\
\hline CT-316 Cast & 717 & 3.22 & 1.27 & 1.37 & 1.36 & 4.00 \\
\hline TEM-USU-400-3-36 & 716 & 1.57 & 1.59 & 1.72 & 1.70 & 5.01 \\
\hline TEM-USU-400-3-36 & 715 & 1.54 & 1.60 & 1.73 & 1.71 & 5.04 \\
\hline TEM-USU-400-3-36 & 714 & 1.51 & 1.57 & 1.70 & 1.68 & 4.95 \\
\hline TEM-USU-400-3-28 & 713 & 1.48 & 1.70 & 1.84 & 1.82 & 5.36 \\
\hline
\end{tabular}


BSU-8242 3 DPA As-Run Physics Analysis

\begin{tabular}{|l|c|c|c|c|c|c|}
\hline \multicolumn{7}{|c|}{ A-7 Position [400C 3DPA (Y)] } \\
\hline Specimen/Material & $\begin{array}{c}\text { Specimen } \\
\text { Number }\end{array}$ & $\begin{array}{c}\text { Distance } \\
\text { from Core } \\
\text { Midplane } \\
(\mathrm{cm})\end{array}$ & $\begin{array}{c}\text { DPA } \\
\text { Cycle 1 }\end{array}$ & $\begin{array}{c}\text { DPA } \\
\text { Cycle 2 }\end{array}$ & $\begin{array}{c}\text { DPA } \\
\text { Cycle 3 }\end{array}$ & $\begin{array}{c}\text { Total } \\
\text { DPA }\end{array}$ \\
\hline TEM-USU-400-3-28 & 712 & 1.45 & 1.71 & 1.85 & 1.83 & 5.38 \\
\hline TEM-USU-400-3-28 & 711 & 1.42 & 1.71 & 1.85 & 1.83 & 5.38 \\
\hline TEM-USU-400-3-20 & 710 & 1.39 & 1.87 & 2.03 & 2.00 & 5.90 \\
\hline TEM-USU-400-3-20 & 709 & 1.36 & 1.84 & 2.00 & 1.98 & 5.82 \\
\hline TEM-USU-400-3-20 & 708 & 1.33 & 1.81 & 1.96 & 1.94 & 5.72 \\
\hline CT-316 PM-HIP & 707 & -3.22 & 1.27 & 1.37 & 1.36 & 4.00 \\
\hline CT-316 PM-HIP & 706 & -3.22 & 1.26 & 1.37 & 1.35 & 3.99 \\
\hline RT-316L Forged & 705 & -8.89 & 1.25 & 1.35 & 1.34 & 3.94 \\
\hline RT-316L Forged & 704 & -16.51 & 1.22 & 1.32 & 1.30 & 3.84 \\
\hline RT-91 Cast & 703 & -24.13 & 1.12 & 1.22 & 1.20 & 3.54 \\
\hline RT-304L Forged & 702 & -31.75 & 1.07 & 1.15 & 1.14 & 3.36 \\
\hline RT-304L Forged & 701 & -39.37 & 0.95 & 1.02 & 1.01 & 2.98 \\
\hline
\end{tabular}

Table 17. Position A-8 specimen DPA values of the BSU-8242-400 ${ }^{\circ} \mathrm{C} / 3$ DPA (X) capsule analyzed at a Center Lobe Power of 19.7/21.9/22.1 MW per the three cycles.

\begin{tabular}{|l|c|c|c|c|c|c|}
\hline \multicolumn{7}{|c|}{ A-8 Position [400C 3DPA (X)] } \\
\hline Specimen/Material & $\begin{array}{c}\text { Specimen } \\
\text { Number }\end{array}$ & $\begin{array}{c}\text { Distance } \\
\text { from Core } \\
\text { Midplane } \\
(\mathrm{cm})\end{array}$ & $\begin{array}{c}\text { DPA } \\
\text { Cycle 1 }\end{array}$ & $\begin{array}{c}\text { DPA } \\
\text { Cycle 2 }\end{array}$ & $\begin{array}{c}\text { DPA } \\
\text { Cycle 3 }\end{array}$ & $\begin{array}{c}\text { Total } \\
\text { DPA }\end{array}$ \\
\hline RT-690 PM-HIP & 674 & 39.37 & 0.89 & 0.97 & 0.96 & 2.82 \\
\hline RT-690 PM-HIP & 673 & 31.75 & 1.04 & 1.13 & 1.12 & 3.29 \\
\hline RT-625 PM-HIP & 672 & 24.13 & 1.17 & 1.27 & 1.25 & 3.69 \\
\hline RT-625 PM-HIP & 671 & 16.51 & 1.25 & 1.35 & 1.33 & 3.93 \\
\hline RT-91 PM-HIP & 670 & 8.89 & 1.17 & 1.27 & 1.25 & 3.68 \\
\hline CT-625 PM-HIP & 669 & 3.22 & 1.32 & 1.43 & 1.41 & 4.16 \\
\hline CT-625 Cast & 668 & 3.22 & 1.31 & 1.42 & 1.40 & 4.13 \\
\hline TEM-91 Cast & 667 & 1.70 & 1.26 & 1.36 & 1.35 & 3.96 \\
\hline TEM-91 Cast & 666 & 1.69 & 1.26 & 1.37 & 1.35 & 3.98 \\
\hline TEM-91 Cast & 665 & 1.67 & 1.26 & 1.36 & 1.35 & 3.97 \\
\hline TEM-91 Cast & 664 & 1.66 & 1.26 & 1.37 & 1.35 & 3.99 \\
\hline TEM-91 Cast & 663 & 1.64 & 1.24 & 1.34 & 1.33 & 3.91 \\
\hline TEM-91 Cast & 662 & 1.63 & 1.27 & 1.37 & 1.36 & 3.99 \\
\hline TEM-304L Forged & 661 & 1.61 & 1.24 & 1.34 & 1.33 & 3.91 \\
\hline TEM-304L Forged & 660 & 1.60 & 1.26 & 1.36 & 1.35 & 3.96 \\
\hline
\end{tabular}


ENGINEERING CALCULATIONS AND ANALYSIS

ECAR- 4951, Rev. 1

Page 42 of 104

BSU-8242 3 DPA As-Run Physics Analysis

\begin{tabular}{|c|c|c|c|c|c|c|}
\hline \multicolumn{6}{|c|}{ A-8 Position [400C 3DPA (X)] } & \multirow[b]{2}{*}{$\begin{array}{l}\text { Total } \\
\text { DPA }\end{array}$} \\
\hline Specimen/Material & $\begin{array}{l}\text { Specimen } \\
\text { Number }\end{array}$ & $\begin{array}{l}\text { Distance } \\
\text { from Core } \\
\text { Midplane } \\
(\mathrm{cm})\end{array}$ & $\begin{array}{c}\text { DPA } \\
\text { Cycle } 1\end{array}$ & $\begin{array}{c}\text { DPA } \\
\text { Cycle } 2\end{array}$ & $\begin{array}{c}\text { DPA } \\
\text { Cycle } 3\end{array}$ & \\
\hline TEM-304L Forged & 659 & 1.58 & 1.30 & 1.41 & 1.40 & 4.11 \\
\hline TEM-304L Forged & 658 & 1.57 & 1.28 & 1.38 & 1.37 & 4.03 \\
\hline TEM-304L Forged & 657 & 1.55 & 1.28 & 1.39 & 1.38 & 4.05 \\
\hline TEM-304L Forged & 656 & 1.54 & 1.27 & 1.38 & 1.36 & 4.02 \\
\hline TEM-304L Forged & 655 & 1.52 & 1.25 & 1.36 & 1.34 & 3.95 \\
\hline TEM-304L Forged & 654 & 1.51 & 1.25 & 1.36 & 1.34 & 3.95 \\
\hline TEM-304L Forged & 653 & 1.49 & 1.23 & 1.33 & 1.32 & 3.87 \\
\hline TEM-304L Forged & 652 & 1.48 & 1.28 & 1.39 & 1.37 & 4.03 \\
\hline TEM-304L Forged & 651 & 1.46 & 1.27 & 1.37 & 1.36 & 4.00 \\
\hline TEM-304L Forged & 650 & 1.45 & 1.28 & 1.38 & 1.37 & 4.03 \\
\hline TEM-316L PM-HIP & 649 & 1.43 & 1.26 & 1.37 & 1.35 & 3.98 \\
\hline TEM-316L PM-HIP & 648 & 1.42 & 1.26 & 1.37 & 1.35 & 3.98 \\
\hline TEM-316L PM-HIP & 647 & 1.40 & 1.25 & 1.36 & 1.34 & 3.95 \\
\hline TEM-316L PM-HIP & 646 & 1.39 & 1.26 & 1.36 & 1.35 & 3.96 \\
\hline TEM-316L PM-HIP & 645 & 1.37 & 1.27 & 1.38 & 1.36 & 4.00 \\
\hline TEM-316L PM-HIP & 644 & 1.36 & 1.31 & 1.42 & 1.40 & 4.13 \\
\hline TEM-316L Forged & 643 & 1.34 & 1.25 & 1.36 & 1.34 & 3.95 \\
\hline TEM-316L Forged & 642 & 1.33 & 1.23 & 1.33 & 1.32 & 3.88 \\
\hline TEM-316L Forged & 641 & 1.31 & 1.24 & 1.34 & 1.33 & 3.91 \\
\hline TEM-316L Forged & 640 & 1.30 & 1.21 & 1.31 & 1.29 & 3.81 \\
\hline TEM-316L Forged & 639 & 1.28 & 1.18 & 1.28 & 1.27 & 3.73 \\
\hline TEM-316L Forged & 638 & 1.27 & 1.17 & 1.27 & 1.25 & 3.69 \\
\hline TEM-91 PM-HIP & 637 & -1.29 & 1.17 & 1.27 & 1.25 & 3.70 \\
\hline TEM-91 PM-HIP & 636 & -1.30 & 1.24 & 1.35 & 1.33 & 3.92 \\
\hline TEM-91 PM-HIP & 635 & -1.32 & 1.20 & 1.30 & 1.28 & 3.78 \\
\hline TEM-91 PM-HIP & 634 & -1.33 & 1.20 & 1.30 & 1.29 & 3.80 \\
\hline TEM-91 PM-HIP & 633 & -1.35 & 1.15 & 1.25 & 1.24 & 3.64 \\
\hline TEM-91 PM-HIP & 632 & -1.36 & 1.14 & 1.24 & 1.22 & 3.60 \\
\hline TEM-690 Forged & 631 & -1.38 & 1.27 & 1.38 & 1.36 & 4.02 \\
\hline TEM-690 Forged & 630 & -1.39 & 1.29 & 1.40 & 1.39 & 4.09 \\
\hline TEM-690 Forged & 629 & -1.41 & 1.31 & 1.42 & 1.40 & 4.14 \\
\hline TEM-690 Forged & 628 & -1.42 & 1.34 & 1.45 & 1.44 & 4.23 \\
\hline TEM-690 Forged & 627 & -1.44 & 1.29 & 1.40 & 1.38 & 4.07 \\
\hline
\end{tabular}


ENGINEERING CALCULATIONS AND ANALYSIS

BSU-8242 3 DPA As-Run Physics Analysis
ECAR- 4951, Rev. 1

Page 43 of 104

\begin{tabular}{|c|c|c|c|c|c|c|}
\hline \multicolumn{6}{|c|}{ A-8 Position [400C 3DPA (X)] } & \multirow[b]{2}{*}{$\begin{array}{l}\text { Total } \\
\text { DPA }\end{array}$} \\
\hline Specimen/Material & $\begin{array}{l}\text { Specimen } \\
\text { Number }\end{array}$ & $\begin{array}{l}\text { Distance } \\
\text { from Core } \\
\text { Midplane } \\
(\mathrm{cm})\end{array}$ & $\begin{array}{c}\text { DPA } \\
\text { Cycle } 1\end{array}$ & $\begin{array}{c}\text { DPA } \\
\text { Cycle } 2\end{array}$ & $\begin{array}{c}\text { DPA } \\
\text { Cycle } 3\end{array}$ & \\
\hline TEM-690 Forged & 626 & -1.45 & 1.30 & 1.41 & 1.40 & 4.11 \\
\hline TEM-690 PM-HIP & 625 & -1.47 & 1.33 & 1.44 & 1.43 & 4.20 \\
\hline TEM-690 PM-HIP & 624 & -1.48 & 1.33 & 1.44 & 1.42 & 4.18 \\
\hline TEM-690 PM-HIP & 623 & -1.50 & 1.28 & 1.39 & 1.37 & 4.05 \\
\hline TEM-690 PM-HIP & 622 & -1.51 & 1.30 & 1.41 & 1.40 & 4.11 \\
\hline TEM-690 PM-HIP & 621 & -1.53 & 1.30 & 1.41 & 1.40 & 4.11 \\
\hline TEM-690 PM-HIP & 620 & -1.54 & 1.33 & 1.44 & 1.42 & 4.18 \\
\hline TEM-625 Forged & 619 & -1.56 & 1.34 & 1.45 & 1.43 & 4.22 \\
\hline TEM-625 Forged & 618 & -1.57 & 1.34 & 1.45 & 1.43 & 4.21 \\
\hline TEM-625 Forged & 617 & -1.59 & 1.37 & 1.48 & 1.46 & 4.31 \\
\hline TEM-625 Forged & 616 & -1.60 & 1.35 & 1.46 & 1.44 & 4.25 \\
\hline TEM-625 Forged & 615 & -1.62 & 1.31 & 1.42 & 1.40 & 4.13 \\
\hline TEM-625 Forged & 614 & -1.63 & 1.29 & 1.40 & 1.38 & 4.06 \\
\hline TEM-625 PM-HIP & 613 & -1.65 & 1.33 & 1.44 & 1.42 & 4.19 \\
\hline TEM-625 PM-HIP & 612 & -1.66 & 1.32 & 1.43 & 1.42 & 4.17 \\
\hline TEM-625 PM-HIP & 611 & -1.68 & 1.35 & 1.47 & 1.45 & 4.28 \\
\hline TEM-625 PM-HIP & 610 & -1.69 & 1.35 & 1.46 & 1.44 & 4.25 \\
\hline TEM-625 PM-HIP & 609 & -1.71 & 1.37 & 1.49 & 1.47 & 4.33 \\
\hline TEM-625 PM-HIP & 608 & -1.72 & 1.39 & 1.51 & 1.49 & 4.40 \\
\hline CT-690 PM-HIP & 607 & -3.22 & 1.32 & 1.43 & 1.41 & 4.16 \\
\hline CT-690 PM-HIP & 606 & -3.22 & 1.32 & 1.43 & 1.42 & 4.17 \\
\hline RT-91 Cast & 605 & -8.89 & 1.18 & 1.28 & 1.26 & 3.71 \\
\hline RT-625 Forged & 604 & -16.51 & 1.27 & 1.38 & 1.36 & 4.01 \\
\hline RT-625 Forged & 603 & -24.13 & 1.22 & 1.32 & 1.30 & 3.84 \\
\hline RT-690 Forged & 602 & -31.75 & 1.12 & 1.21 & 1.19 & 3.52 \\
\hline RT-690 Forged & 601 & -39.37 & 0.99 & 1.07 & 1.06 & 3.12 \\
\hline
\end{tabular}




\subsection{Decay Heat}

Table 18 provides the decay heat (in watts per capsule) for each of the 3 DPA BSU-8242 capsules after the end of the final irradiation cycle (166B), with additional time periods over the subsequent year. Calculations were made with the use of the ATR as-run data for lobe powers, irradiation periods, and outages, as summarized in Table 5.

Table 18. Decay Heating of the 3DPA Capsules Post Cycle 166B Irradiation.

\begin{tabular}{|c|c|c|c|c|c|c|c|c|c|}
\hline \multicolumn{10}{|c|}{ DECAY HEAT (Watts/Capsule) } \\
\hline & EOC & 1 Day & 2 Days & 10 Days & 30 Days & 90 Days & 130 Days & 180 Days & 360 Days \\
\hline A6 300C 3 DPA & 72.01 & 26.27 & 23.65 & 12.19 & 6.26 & 4.41 & 3.78 & 3.22 & 2.21 \\
\hline A7 400C 3 DPA Y & 109.00 & 34.72 & 30.90 & 14.09 & 5.53 & 3.32 & 2.58 & 1.91 & 0.68 \\
\hline A8 400C 3 DPA X & 87.92 & 36.60 & 32.84 & 16.24 & 7.71 & 5.23 & 4.38 & 3.61 & 2.22 \\
\hline
\end{tabular}




\subsection{Radioactivity}

The following six tables provide the total radionuclide activity in curies per capsule (Table 19-21) and mass per capsule (Table 22-24) detailed isotopically for each of the three DPA BSU-8242 capsules, which includes all material components, following the irradiation of Cycle 166B and carried out in eight additional increments over the subsequent year. Calculations were made with the implementation, within the ORIGEN input deck, of the ATR as-run lobe power data and irradiation times for Cycle 164B, 166A, and 166B. See Table 19 for the 'A6 300C 3 DPA' capsule source terms, Table 20 for the 'A7 400C 3 DPA X' capsule source terms, and Table 21 for the 'A8 400C 3 DPA Y' capsule source terms.

Table 19. Source Terms for 'A6 300C 3 DPA' Capsule.

\begin{tabular}{|c|c|c|c|c|c|c|c|c|c|}
\hline \multicolumn{10}{|c|}{ ACTIVATION PRODUCTS (Ci) } \\
\hline ISOTOPE & EOC & 1 Day & 2 Days & 10 Days & 30 Days & 90 Days & 130 Days & 180 Days & 360 Days \\
\hline H 3 & $8.72 \mathrm{E}-05$ & $8.72 \mathrm{E}-05$ & $8.72 \mathrm{E}-05$ & $8.71 \mathrm{E}-05$ & 8.68E-05 & $8.60 \mathrm{E}-05$ & $8.55 \mathrm{E}-05$ & $8.48 \mathrm{E}-05$ & $8.25 \mathrm{E}-05$ \\
\hline $\mathrm{H} 4$ & $2.79 \mathrm{E}-11$ & $0.00 \mathrm{E}+00$ & $0.00 \mathrm{E}+00$ & $0.00 \mathrm{E}+00$ & $0.00 \mathrm{E}+00$ & $0.00 \mathrm{E}+00$ & $0.00 \mathrm{E}+00$ & $0.00 \mathrm{E}+00$ & $0.00 \mathrm{E}+00$ \\
\hline HE 6 & $2.06 \mathrm{E}-04$ & $0.00 \mathrm{E}+00$ & $0.00 \mathrm{E}+00$ & $0.00 \mathrm{E}+00$ & $0.00 \mathrm{E}+00$ & $0.00 \mathrm{E}+00$ & $0.00 \mathrm{E}+00$ & $0.00 \mathrm{E}+00$ & $0.00 \mathrm{E}+00$ \\
\hline LI 8 & $1.05 \mathrm{E}-13$ & $0.00 \mathrm{E}+00$ & $0.00 \mathrm{E}+00$ & $0.00 \mathrm{E}+00$ & $0.00 \mathrm{E}+00$ & $0.00 \mathrm{E}+00$ & $0.00 \mathrm{E}+00$ & $0.00 \mathrm{E}+00$ & $0.00 \mathrm{E}+00$ \\
\hline BE 8 & $3.55 \mathrm{E}-04$ & $0.00 \mathrm{E}+00$ & $0.00 \mathrm{E}+00$ & $0.00 \mathrm{E}+00$ & $0.00 \mathrm{E}+00$ & $0.00 \mathrm{E}+00$ & $0.00 \mathrm{E}+00$ & $0.00 \mathrm{E}+00$ & $0.00 \mathrm{E}+00$ \\
\hline BE 10 & $1.67 \mathrm{E}-09$ & $1.67 \mathrm{E}-09$ & $1.67 \mathrm{E}-09$ & $1.67 \mathrm{E}-09$ & $1.67 \mathrm{E}-09$ & $1.67 \mathrm{E}-09$ & $1.67 \mathrm{E}-09$ & $1.67 \mathrm{E}-09$ & $1.67 \mathrm{E}-09$ \\
\hline BE 11 & $1.14 \mathrm{E}-08$ & $0.00 \mathrm{E}+00$ & $0.00 \mathrm{E}+00$ & $0.00 \mathrm{E}+00$ & $0.00 \mathrm{E}+00$ & $0.00 \mathrm{E}+00$ & $0.00 \mathrm{E}+00$ & $0.00 \mathrm{E}+00$ & $0.00 \mathrm{E}+00$ \\
\hline B 12 & $4.86 \mathrm{E}-14$ & $0.00 \mathrm{E}+00$ & $0.00 \mathrm{E}+00$ & $0.00 \mathrm{E}+00$ & $0.00 \mathrm{E}+00$ & $0.00 \mathrm{E}+00$ & $0.00 \mathrm{E}+00$ & $0.00 \mathrm{E}+00$ & $0.00 \mathrm{E}+00$ \\
\hline C 14 & $6.13 \mathrm{E}-08$ & $6.13 \mathrm{E}-08$ & $6.13 \mathrm{E}-08$ & $6.13 \mathrm{E}-08$ & $6.13 \mathrm{E}-08$ & $6.13 \mathrm{E}-08$ & $6.13 \mathrm{E}-08$ & $6.13 \mathrm{E}-08$ & $6.13 \mathrm{E}-08$ \\
\hline C 15 & $1.61 \mathrm{E}-09$ & $0.00 \mathrm{E}+00$ & $0.00 \mathrm{E}+00$ & $0.00 \mathrm{E}+00$ & $0.00 \mathrm{E}+00$ & $0.00 \mathrm{E}+00$ & $0.00 \mathrm{E}+00$ & $0.00 \mathrm{E}+00$ & $0.00 \mathrm{E}+00$ \\
\hline N 16 & $3.22 \mathrm{E}-15$ & $0.00 \mathrm{E}+00$ & $0.00 \mathrm{E}+00$ & $0.00 \mathrm{E}+00$ & $0.00 \mathrm{E}+00$ & $0.00 \mathrm{E}+00$ & $0.00 \mathrm{E}+00$ & $0.00 \mathrm{E}+00$ & $0.00 \mathrm{E}+00$ \\
\hline O 19 & $1.15 \mathrm{E}-10$ & $0.00 \mathrm{E}+00$ & $0.00 \mathrm{E}+00$ & $0.00 \mathrm{E}+00$ & $0.00 \mathrm{E}+00$ & $0.00 \mathrm{E}+00$ & $0.00 \mathrm{E}+00$ & $0.00 \mathrm{E}+00$ & $0.00 \mathrm{E}+00$ \\
\hline F 20 & $1.53 \mathrm{E}-07$ & $0.00 \mathrm{E}+00$ & $0.00 \mathrm{E}+00$ & $0.00 \mathrm{E}+00$ & $0.00 \mathrm{E}+00$ & $0.00 \mathrm{E}+00$ & $0.00 \mathrm{E}+00$ & $0.00 \mathrm{E}+00$ & $0.00 \mathrm{E}+00$ \\
\hline NE 23 & $5.61 \mathrm{E}-02$ & $0.00 \mathrm{E}+00$ & $0.00 \mathrm{E}+00$ & $0.00 \mathrm{E}+00$ & $0.00 \mathrm{E}+00$ & $0.00 \mathrm{E}+00$ & $0.00 \mathrm{E}+00$ & $0.00 \mathrm{E}+00$ & $0.00 \mathrm{E}+00$ \\
\hline NA 24 & $1.38 \mathrm{E}+01$ & $4.56 \mathrm{E}+00$ & $1.50 \mathrm{E}+00$ & $2.11 \mathrm{E}-04$ & $4.91 \mathrm{E}-14$ & $0.00 \mathrm{E}+00$ & $0.00 \mathrm{E}+00$ & $0.00 \mathrm{E}+00$ & $0.00 \mathrm{E}+00$ \\
\hline NA $24 \mathrm{M}$ & $3.40 \mathrm{E}-05$ & $0.00 \mathrm{E}+00$ & $0.00 \mathrm{E}+00$ & $0.00 \mathrm{E}+00$ & $0.00 \mathrm{E}+00$ & $0.00 \mathrm{E}+00$ & $0.00 \mathrm{E}+00$ & $0.00 \mathrm{E}+00$ & $0.00 \mathrm{E}+00$ \\
\hline NA 25 & $3.39 \mathrm{E}-02$ & $0.00 \mathrm{E}+00$ & $0.00 \mathrm{E}+00$ & $0.00 \mathrm{E}+00$ & $0.00 \mathrm{E}+00$ & $0.00 \mathrm{E}+00$ & $0.00 \mathrm{E}+00$ & $0.00 \mathrm{E}+00$ & $0.00 \mathrm{E}+00$ \\
\hline MG 27 & $6.55 \mathrm{E}+01$ & $0.00 \mathrm{E}+00$ & $0.00 \mathrm{E}+00$ & $0.00 \mathrm{E}+00$ & $0.00 \mathrm{E}+00$ & $0.00 \mathrm{E}+00$ & $0.00 \mathrm{E}+00$ & $0.00 \mathrm{E}+00$ & $0.00 \mathrm{E}+00$ \\
\hline MG 28 & $2.01 \mathrm{E}-07$ & $9.08 \mathrm{E}-08$ & $4.10 \mathrm{E}-08$ & $7.06 \mathrm{E}-11$ & 8.69E-18 & $0.00 \mathrm{E}+00$ & $0.00 \mathrm{E}+00$ & $0.00 \mathrm{E}+00$ & $0.00 \mathrm{E}+00$ \\
\hline
\end{tabular}


BSU-8242 3 DPA As-Run Physics Analysis

\begin{tabular}{|c|c|c|c|c|c|c|c|c|c|}
\hline \multicolumn{10}{|c|}{ ACTIVATION PRODUCTS (Ci) } \\
\hline ISOTOPE & EOC & 1 Day & 2 Days & 10 Days & 30 Days & 90 Days & 130 Days & 180 Days & 360 Days \\
\hline AL 28 & $1.57 \mathrm{E}+03$ & $9.10 \mathrm{E}-08$ & $4.11 \mathrm{E}-08$ & $7.07 \mathrm{E}-11$ & $8.70 \mathrm{E}-18$ & $0.00 \mathrm{E}+00$ & $0.00 \mathrm{E}+00$ & $0.00 \mathrm{E}+00$ & $0.00 \mathrm{E}+00$ \\
\hline AL 29 & $3.23 \mathrm{E}-02$ & $0.00 \mathrm{E}+00$ & $0.00 \mathrm{E}+00$ & $0.00 \mathrm{E}+00$ & $0.00 \mathrm{E}+00$ & $0.00 \mathrm{E}+00$ & $0.00 \mathrm{E}+00$ & $0.00 \mathrm{E}+00$ & $0.00 \mathrm{E}+00$ \\
\hline AL 30 & $3.12 \mathrm{E}-05$ & $0.00 \mathrm{E}+00$ & $0.00 \mathrm{E}+00$ & $0.00 \mathrm{E}+00$ & $0.00 \mathrm{E}+00$ & $0.00 \mathrm{E}+00$ & $0.00 \mathrm{E}+00$ & $0.00 \mathrm{E}+00$ & $0.00 \mathrm{E}+00$ \\
\hline SI 31 & $3.39 \mathrm{E}-01$ & 5.93E-04 & $1.04 \mathrm{E}-06$ & $0.00 \mathrm{E}+00$ & $0.00 \mathrm{E}+00$ & $0.00 \mathrm{E}+00$ & $0.00 \mathrm{E}+00$ & $0.00 \mathrm{E}+00$ & $0.00 \mathrm{E}+00$ \\
\hline SI 32 & $1.05 \mathrm{E}-10$ & $1.05 \mathrm{E}-10$ & $1.05 \mathrm{E}-10$ & $1.05 \mathrm{E}-10$ & $1.05 \mathrm{E}-10$ & $1.05 \mathrm{E}-10$ & $1.05 \mathrm{E}-10$ & $1.05 \mathrm{E}-10$ & $1.05 \mathrm{E}-10$ \\
\hline P 32 & $2.41 \mathrm{E}-01$ & $2.30 \mathrm{E}-01$ & $2.19 \mathrm{E}-01$ & $1.49 \mathrm{E}-01$ & $5.64 \mathrm{E}-02$ & $3.08 \mathrm{E}-03$ & $4.43 \mathrm{E}-04$ & $3.92 \mathrm{E}-05$ & $6.48 \mathrm{E}-09$ \\
\hline P 33 & $1.26 \mathrm{E}-05$ & $1.22 \mathrm{E}-05$ & $1.19 \mathrm{E}-05$ & $9.53 \mathrm{E}-06$ & $5.47 \mathrm{E}-06$ & $1.04 \mathrm{E}-06$ & $3.42 \mathrm{E}-07$ & $8.55 \mathrm{E}-08$ & $5.82 \mathrm{E}-10$ \\
\hline P 34 & $7.81 \mathrm{E}-05$ & $0.00 \mathrm{E}+00$ & $0.00 \mathrm{E}+00$ & $0.00 \mathrm{E}+00$ & $0.00 \mathrm{E}+00$ & $0.00 \mathrm{E}+00$ & $0.00 \mathrm{E}+00$ & $0.00 \mathrm{E}+00$ & $0.00 \mathrm{E}+00$ \\
\hline S 35 & $5.25 \mathrm{E}-03$ & $5.21 \mathrm{E}-03$ & $5.17 \mathrm{E}-03$ & $4.85 \mathrm{E}-03$ & $4.15 \mathrm{E}-03$ & $2.59 \mathrm{E}-03$ & $1.89 \mathrm{E}-03$ & $1.27 \mathrm{E}-03$ & $3.08 \mathrm{E}-04$ \\
\hline S 37 & $2.26 \mathrm{E}-05$ & $0.00 \mathrm{E}+00$ & $0.00 \mathrm{E}+00$ & $0.00 \mathrm{E}+00$ & $0.00 \mathrm{E}+00$ & $0.00 \mathrm{E}+00$ & $0.00 \mathrm{E}+00$ & $0.00 \mathrm{E}+00$ & $0.00 \mathrm{E}+00$ \\
\hline CL 36 & $1.72 \mathrm{E}-10$ & $1.72 \mathrm{E}-10$ & $1.72 \mathrm{E}-10$ & $1.72 \mathrm{E}-10$ & $1.72 \mathrm{E}-10$ & $1.72 \mathrm{E}-10$ & $1.72 \mathrm{E}-10$ & $1.72 \mathrm{E}-10$ & $1.72 \mathrm{E}-10$ \\
\hline CL 38 & $1.56 \mathrm{E}-08$ & $3.51 \mathrm{E}-20$ & $6.85 \mathrm{E}-32$ & $0.00 \mathrm{E}+00$ & $0.00 \mathrm{E}+00$ & $0.00 \mathrm{E}+00$ & $0.00 \mathrm{E}+00$ & $0.00 \mathrm{E}+00$ & $0.00 \mathrm{E}+00$ \\
\hline CL 38M & $1.86 \mathrm{E}-10$ & $0.00 \mathrm{E}+00$ & $0.00 \mathrm{E}+00$ & $0.00 \mathrm{E}+00$ & $0.00 \mathrm{E}+00$ & $0.00 \mathrm{E}+00$ & $0.00 \mathrm{E}+00$ & $0.00 \mathrm{E}+00$ & $0.00 \mathrm{E}+00$ \\
\hline AR 37 & $2.69 \mathrm{E}-13$ & $2.64 \mathrm{E}-13$ & $2.59 \mathrm{E}-13$ & $2.21 \mathrm{E}-13$ & $1.49 \mathrm{E}-13$ & $4.53 \mathrm{E}-14$ & $2.05 \mathrm{E}-14$ & $7.63 \mathrm{E}-15$ & $2.16 \mathrm{E}-16$ \\
\hline AR 39 & $3.12 \mathrm{E}-15$ & $3.12 \mathrm{E}-15$ & $3.12 \mathrm{E}-15$ & $3.12 \mathrm{E}-15$ & $3.12 \mathrm{E}-15$ & $3.12 \mathrm{E}-15$ & $3.11 \mathrm{E}-15$ & $3.11 \mathrm{E}-15$ & $3.11 \mathrm{E}-15$ \\
\hline AR 41 & $3.04 \mathrm{E}-09$ & $3.38 \mathrm{E}-13$ & $3.75 \mathrm{E}-17$ & $0.00 \mathrm{E}+00$ & $0.00 \mathrm{E}+00$ & $0.00 \mathrm{E}+00$ & $0.00 \mathrm{E}+00$ & $0.00 \mathrm{E}+00$ & $0.00 \mathrm{E}+00$ \\
\hline AR 42 & $6.84 \mathrm{E}-18$ & $6.84 \mathrm{E}-18$ & $6.84 \mathrm{E}-18$ & $6.84 \mathrm{E}-18$ & $6.83 \mathrm{E}-18$ & $6.81 \mathrm{E}-18$ & $6.79 \mathrm{E}-18$ & $6.77 \mathrm{E}-18$ & $6.70 \mathrm{E}-18$ \\
\hline K 42 & $3.14 \mathrm{E}-11$ & $8.17 \mathrm{E}-12$ & $2.13 \mathrm{E}-12$ & $5.17 \mathrm{E}-17$ & $6.83 \mathrm{E}-18$ & $6.81 \mathrm{E}-18$ & $6.79 \mathrm{E}-18$ & $6.77 \mathrm{E}-18$ & $6.70 \mathrm{E}-18$ \\
\hline K 43 & $3.86 \mathrm{E}-08$ & $1.85 \mathrm{E}-08$ & 8.84E-09 & $2.45 \mathrm{E}-11$ & $9.90 \mathrm{E}-18$ & $0.00 \mathrm{E}+00$ & $0.00 \mathrm{E}+00$ & $0.00 \mathrm{E}+00$ & $0.00 \mathrm{E}+00$ \\
\hline K 44 & $6.85 \mathrm{E}-09$ & $1.35 \mathrm{E}-28$ & $0.00 \mathrm{E}+00$ & $0.00 \mathrm{E}+00$ & $0.00 \mathrm{E}+00$ & $0.00 \mathrm{E}+00$ & $0.00 \mathrm{E}+00$ & $0.00 \mathrm{E}+00$ & $0.00 \mathrm{E}+00$ \\
\hline CA 45 & $1.57 \mathrm{E}-04$ & $1.57 \mathrm{E}-04$ & $1.56 \mathrm{E}-04$ & $1.51 \mathrm{E}-04$ & $1.39 \mathrm{E}-04$ & $1.07 \mathrm{E}-04$ & $9.06 \mathrm{E}-05$ & $7.32 \mathrm{E}-05$ & $3.40 \mathrm{E}-05$ \\
\hline CA 47 & $2.98 \mathrm{E}-06$ & $2.56 \mathrm{E}-06$ & $2.20 \mathrm{E}-06$ & $6.47 \mathrm{E}-07$ & $3.04 \mathrm{E}-08$ & $3.17 \mathrm{E}-12$ & $7.03 \mathrm{E}-15$ & $3.38 \mathrm{E}-18$ & $3.83 \mathrm{E}-30$ \\
\hline SC 46 & $2.72 \mathrm{E}-02$ & $2.70 \mathrm{E}-02$ & $2.68 \mathrm{E}-02$ & $2.50 \mathrm{E}-02$ & $2.12 \mathrm{E}-02$ & $1.29 \mathrm{E}-02$ & $9.28 \mathrm{E}-03$ & $6.14 \mathrm{E}-03$ & $1.38 \mathrm{E}-03$ \\
\hline $\mathrm{SC} 46 \mathrm{M}$ & $2.13 \mathrm{E}-06$ & $0.00 \mathrm{E}+00$ & $0.00 \mathrm{E}+00$ & $0.00 \mathrm{E}+00$ & $0.00 \mathrm{E}+00$ & $0.00 \mathrm{E}+00$ & $0.00 \mathrm{E}+00$ & $0.00 \mathrm{E}+00$ & $0.00 \mathrm{E}+00$ \\
\hline SC 47 & $7.05 \mathrm{E}-02$ & $5.74 \mathrm{E}-02$ & $4.66 \mathrm{E}-02$ & 8.91E-03 & $1.42 \mathrm{E}-04$ & $5.91 \mathrm{E}-10$ & $1.78 \mathrm{E}-13$ & $1.78 \mathrm{E}-17$ & $1.47 \mathrm{E}-29$ \\
\hline $\mathrm{SC} 48$ & $6.61 \mathrm{E}-03$ & $4.52 \mathrm{E}-03$ & $3.09 \mathrm{E}-03$ & $1.48 \mathrm{E}-04$ & $7.45 \mathrm{E}-08$ & $9.48 \mathrm{E}-18$ & $2.40 \mathrm{E}-24$ & $2.28 \mathrm{E}-32$ & $0.00 \mathrm{E}+00$ \\
\hline
\end{tabular}


BSU-8242 3 DPA As-Run Physics Analysis

\begin{tabular}{|c|c|c|c|c|c|c|c|c|c|}
\hline \multicolumn{10}{|c|}{ ACTIVATION PRODUCTS (Ci) } \\
\hline ISOTOPE & EOC & 1 Day & 2 Days & 10 Days & 30 Days & 90 Days & 130 Days & 180 Days & 360 Days \\
\hline SC 49 & $6.16 \mathrm{E}-03$ & $1.78 \mathrm{E}-10$ & $5.15 \mathrm{E}-18$ & $0.00 \mathrm{E}+00$ & $0.00 \mathrm{E}+00$ & $0.00 \mathrm{E}+00$ & $0.00 \mathrm{E}+00$ & $0.00 \mathrm{E}+00$ & $0.00 \mathrm{E}+00$ \\
\hline SC 50 & $1.13 \mathrm{E}-04$ & $0.00 \mathrm{E}+00$ & $0.00 \mathrm{E}+00$ & $0.00 \mathrm{E}+00$ & $0.00 \mathrm{E}+00$ & $0.00 \mathrm{E}+00$ & $0.00 \mathrm{E}+00$ & $0.00 \mathrm{E}+00$ & $0.00 \mathrm{E}+00$ \\
\hline TI 51 & $1.44 \mathrm{E}-01$ & $0.00 \mathrm{E}+00$ & $0.00 \mathrm{E}+00$ & $0.00 \mathrm{E}+00$ & $0.00 \mathrm{E}+00$ & $0.00 \mathrm{E}+00$ & $0.00 \mathrm{E}+00$ & $0.00 \mathrm{E}+00$ & $0.00 \mathrm{E}+00$ \\
\hline V 50 & $1.12 \mathrm{E}-16$ & $1.12 \mathrm{E}-16$ & $1.12 \mathrm{E}-16$ & $1.12 \mathrm{E}-16$ & $1.12 \mathrm{E}-16$ & $1.12 \mathrm{E}-16$ & $1.12 \mathrm{E}-16$ & $1.12 \mathrm{E}-16$ & $1.12 \mathrm{E}-16$ \\
\hline V 52 & $3.18 \mathrm{E}+01$ & $0.00 \mathrm{E}+00$ & $0.00 \mathrm{E}+00$ & $0.00 \mathrm{E}+00$ & $0.00 \mathrm{E}+00$ & $0.00 \mathrm{E}+00$ & $0.00 \mathrm{E}+00$ & $0.00 \mathrm{E}+00$ & $0.00 \mathrm{E}+00$ \\
\hline V 53 & $1.22 \mathrm{E}-01$ & $0.00 \mathrm{E}+00$ & $0.00 \mathrm{E}+00$ & $0.00 \mathrm{E}+00$ & $0.00 \mathrm{E}+00$ & $0.00 \mathrm{E}+00$ & $0.00 \mathrm{E}+00$ & $0.00 \mathrm{E}+00$ & $0.00 \mathrm{E}+00$ \\
\hline V 54 & $1.33 \mathrm{E}-03$ & $0.00 \mathrm{E}+00$ & $0.00 \mathrm{E}+00$ & $0.00 \mathrm{E}+00$ & $0.00 \mathrm{E}+00$ & $0.00 \mathrm{E}+00$ & $0.00 \mathrm{E}+00$ & $0.00 \mathrm{E}+00$ & $0.00 \mathrm{E}+00$ \\
\hline CR 51 & $2.23 \mathrm{E}+03$ & $2.17 \mathrm{E}+03$ & $2.12 \mathrm{E}+03$ & $1.74 \mathrm{E}+03$ & $1.05 \mathrm{E}+03$ & $2.35 \mathrm{E}+02$ & $8.63 \mathrm{E}+01$ & $2.47 \mathrm{E}+01$ & $2.74 \mathrm{E}-01$ \\
\hline CR 55 & $3.65 \mathrm{E}+01$ & $0.00 \mathrm{E}+00$ & $0.00 \mathrm{E}+00$ & $0.00 \mathrm{E}+00$ & $0.00 \mathrm{E}+00$ & $0.00 \mathrm{E}+00$ & $0.00 \mathrm{E}+00$ & $0.00 \mathrm{E}+00$ & $0.00 \mathrm{E}+00$ \\
\hline MN 54 & $2.53 \mathrm{E}+01$ & $2.52 \mathrm{E}+01$ & $2.52 \mathrm{E}+01$ & $2.48 \mathrm{E}+01$ & $2.37 \mathrm{E}+01$ & $2.07 \mathrm{E}+01$ & $1.90 \mathrm{E}+01$ & $1.70 \mathrm{E}+01$ & $1.14 \mathrm{E}+01$ \\
\hline MN 56 & $7.70 \mathrm{E}+02$ & $1.22 \mathrm{E}+00$ & $1.92 \mathrm{E}-03$ & $0.00 \mathrm{E}+00$ & $0.00 \mathrm{E}+00$ & $0.00 \mathrm{E}+00$ & $0.00 \mathrm{E}+00$ & $0.00 \mathrm{E}+00$ & $0.00 \mathrm{E}+00$ \\
\hline MN 57 & $2.37 \mathrm{E}-01$ & $0.00 \mathrm{E}+00$ & $0.00 \mathrm{E}+00$ & $0.00 \mathrm{E}+00$ & $0.00 \mathrm{E}+00$ & $0.00 \mathrm{E}+00$ & $0.00 \mathrm{E}+00$ & $0.00 \mathrm{E}+00$ & $0.00 \mathrm{E}+00$ \\
\hline MN 58 & $7.08 \mathrm{E}-04$ & $0.00 \mathrm{E}+00$ & $0.00 \mathrm{E}+00$ & $0.00 \mathrm{E}+00$ & $0.00 \mathrm{E}+00$ & $0.00 \mathrm{E}+00$ & $0.00 \mathrm{E}+00$ & $0.00 \mathrm{E}+00$ & $0.00 \mathrm{E}+00$ \\
\hline FE 55 & $1.15 \mathrm{E}+02$ & $1.15 \mathrm{E}+02$ & $1.15 \mathrm{E}+02$ & $1.14 \mathrm{E}+02$ & $1.13 E+02$ & $1.08 \mathrm{E}+02$ & $1.05 \mathrm{E}+02$ & $1.01 \mathrm{E}+02$ & $8.84 \mathrm{E}+01$ \\
\hline FE 59 & $2.12 \mathrm{E}+01$ & $2.08 \mathrm{E}+01$ & $2.05 \mathrm{E}+01$ & $1.81 \mathrm{E}+01$ & $1.33 \mathrm{E}+01$ & $5.29 \mathrm{E}+00$ & $2.86 \mathrm{E}+00$ & $1.32 \mathrm{E}+00$ & $8.27 \mathrm{E}-02$ \\
\hline CO 58 & $2.11 \mathrm{E}+02$ & $2.09 \mathrm{E}+02$ & $2.07 \mathrm{E}+02$ & $1.92 \mathrm{E}+02$ & $1.57 \mathrm{E}+02$ & $8.75 \mathrm{E}+01$ & $5.91 \mathrm{E}+01$ & $3.62 \mathrm{E}+01$ & $6.21 \mathrm{E}+00$ \\
\hline $\mathrm{CO} 60$ & $1.31 \mathrm{E}+02$ & $1.31 \mathrm{E}+02$ & $1.31 \mathrm{E}+02$ & $1.31 \mathrm{E}+02$ & $1.30 \mathrm{E}+02$ & $1.27 \mathrm{E}+02$ & $1.25 \mathrm{E}+02$ & $1.23 \mathrm{E}+02$ & $1.15 \mathrm{E}+02$ \\
\hline $\mathrm{CO} 60 \mathrm{M}$ & $1.13 \mathrm{E}+03$ & $0.00 \mathrm{E}+00$ & $0.00 \mathrm{E}+00$ & $0.00 \mathrm{E}+00$ & $0.00 \mathrm{E}+00$ & $0.00 \mathrm{E}+00$ & $0.00 \mathrm{E}+00$ & $0.00 \mathrm{E}+00$ & $0.00 \mathrm{E}+00$ \\
\hline $\mathrm{CO} 61$ & $8.98 \mathrm{E}+00$ & $3.75 \mathrm{E}-04$ & $1.57 \mathrm{E}-08$ & $0.00 \mathrm{E}+00$ & $0.00 \mathrm{E}+00$ & $0.00 \mathrm{E}+00$ & $0.00 \mathrm{E}+00$ & $0.00 \mathrm{E}+00$ & $0.00 \mathrm{E}+00$ \\
\hline $\mathrm{CO} 62$ & $6.85 \mathrm{E}-02$ & $0.00 \mathrm{E}+00$ & $0.00 \mathrm{E}+00$ & $0.00 \mathrm{E}+00$ & $0.00 \mathrm{E}+00$ & $0.00 \mathrm{E}+00$ & $0.00 \mathrm{E}+00$ & $0.00 \mathrm{E}+00$ & $0.00 \mathrm{E}+00$ \\
\hline NI 59 & 7.71E-02 & $7.71 \mathrm{E}-02$ & $7.71 \mathrm{E}-02$ & $7.71 \mathrm{E}-02$ & $7.71 \mathrm{E}-02$ & $7.71 \mathrm{E}-02$ & 7.71E-02 & $7.71 \mathrm{E}-02$ & $7.71 \mathrm{E}-02$ \\
\hline NI 63 & $1.02 \mathrm{E}+01$ & $1.02 \mathrm{E}+01$ & $1.02 \mathrm{E}+01$ & $1.02 \mathrm{E}+01$ & $1.02 \mathrm{E}+01$ & $1.02 \mathrm{E}+01$ & $1.02 \mathrm{E}+01$ & $1.02 \mathrm{E}+01$ & $1.01 \mathrm{E}+01$ \\
\hline NI 65 & $8.04 \mathrm{E}+01$ & $1.09 \mathrm{E}-01$ & $1.48 \mathrm{E}-04$ & $0.00 \mathrm{E}+00$ & $0.00 \mathrm{E}+00$ & $0.00 \mathrm{E}+00$ & $0.00 \mathrm{E}+00$ & $0.00 \mathrm{E}+00$ & $0.00 \mathrm{E}+00$ \\
\hline NI 66 & $2.40 \mathrm{E}-03$ & $1.77 \mathrm{E}-03$ & $1.31 \mathrm{E}-03$ & $1.14 \mathrm{E}-04$ & $2.58 \mathrm{E}-07$ & $2.98 \mathrm{E}-15$ & $1.52 \mathrm{E}-20$ & $3.70 \mathrm{E}-27$ & $0.00 \mathrm{E}+00$ \\
\hline CU 64 & $9.28 \mathrm{E}+01$ & $2.50 \mathrm{E}+01$ & $6.75 \mathrm{E}+00$ & $1.90 \mathrm{E}-04$ & $7.96 \mathrm{E}-16$ & $0.00 \mathrm{E}+00$ & $0.00 \mathrm{E}+00$ & $0.00 \mathrm{E}+00$ & $0.00 \mathrm{E}+00$ \\
\hline CU 66 & $2.04 \mathrm{E}+01$ & $1.77 \mathrm{E}-03$ & $1.31 \mathrm{E}-03$ & $1.14 \mathrm{E}-04$ & $2.59 \mathrm{E}-07$ & $2.99 \mathrm{E}-15$ & $1.53 \mathrm{E}-20$ & $3.71 \mathrm{E}-27$ & $0.00 \mathrm{E}+00$ \\
\hline
\end{tabular}


BSU-8242 3 DPA As-Run Physics Analysis

\begin{tabular}{|c|c|c|c|c|c|c|c|c|c|}
\hline \multicolumn{10}{|c|}{ ACTIVATION PRODUCTS (Ci) } \\
\hline ISOTOPE & EOC & 1 Day & 2 Days & 10 Days & 30 Days & 90 Days & 130 Days & 180 Days & 360 Days \\
\hline CU 67 & $1.14 \mathrm{E}-04$ & $8.70 \mathrm{E}-05$ & $6.65 \mathrm{E}-05$ & $7.73 \mathrm{E}-06$ & $3.57 \mathrm{E}-08$ & $3.51 \mathrm{E}-15$ & $7.48 \mathrm{E}-20$ & $1.08 \mathrm{E}-25$ & $0.00 \mathrm{E}+00$ \\
\hline ZN 65 & $1.09 \mathrm{E}-02$ & $1.08 \mathrm{E}-02$ & $1.08 \mathrm{E}-02$ & $1.06 \mathrm{E}-02$ & $9.98 \mathrm{E}-03$ & $8.41 \mathrm{E}-03$ & $7.51 \mathrm{E}-03$ & $6.51 \mathrm{E}-03$ & $3.91 \mathrm{E}-03$ \\
\hline ZN 69 & $2.11 \mathrm{E}-07$ & $4.55 \mathrm{E}-09$ & $1.36 \mathrm{E}-09$ & $8.58 \mathrm{E}-14$ & $2.71 \mathrm{E}-24$ & $0.00 \mathrm{E}+00$ & $0.00 \mathrm{E}+00$ & $0.00 \mathrm{E}+00$ & $0.00 \mathrm{E}+00$ \\
\hline $\mathrm{ZN} \mathrm{69M}$ & $1.42 \mathrm{E}-08$ & 4.24E-09 & $1.27 \mathrm{E}-09$ & $7.99 \mathrm{E}-14$ & $2.53 \mathrm{E}-24$ & $0.00 \mathrm{E}+00$ & $0.00 \mathrm{E}+00$ & $0.00 \mathrm{E}+00$ & $0.00 \mathrm{E}+00$ \\
\hline GA 70 & 4.37E-10 & $1.26 \mathrm{E}-30$ & $0.00 \mathrm{E}+00$ & $0.00 \mathrm{E}+00$ & $0.00 \mathrm{E}+00$ & $0.00 \mathrm{E}+00$ & $0.00 \mathrm{E}+00$ & $0.00 \mathrm{E}+00$ & $0.00 \mathrm{E}+00$ \\
\hline GA 72 & $9.03 \mathrm{E}-16$ & $2.92 \mathrm{E}-16$ & $9.43 \mathrm{E}-17$ & $7.51 \mathrm{E}-21$ & $4.34 \mathrm{E}-31$ & $0.00 \mathrm{E}+00$ & $0.00 \mathrm{E}+00$ & $0.00 \mathrm{E}+00$ & $0.00 \mathrm{E}+00$ \\
\hline GE 71 & $3.11 \mathrm{E}-13$ & $2.93 \mathrm{E}-13$ & $2.77 \mathrm{E}-13$ & $1.73 \mathrm{E}-13$ & $5.34 \mathrm{E}-14$ & $1.57 \mathrm{E}-15$ & $1.51 \mathrm{E}-16$ & $8.41 \mathrm{E}-18$ & $2.15 \mathrm{E}-22$ \\
\hline GE 71M & $3.83 \mathrm{E}-14$ & $0.00 \mathrm{E}+00$ & $0.00 \mathrm{E}+00$ & $0.00 \mathrm{E}+00$ & $0.00 \mathrm{E}+00$ & $0.00 \mathrm{E}+00$ & $0.00 \mathrm{E}+00$ & $0.00 \mathrm{E}+00$ & $0.00 \mathrm{E}+00$ \\
\hline SR 89 & $1.57 \mathrm{E}-07$ & $1.55 \mathrm{E}-07$ & $1.53 \mathrm{E}-07$ & $1.37 \mathrm{E}-07$ & $1.04 \mathrm{E}-07$ & 4.58E-08 & 2.64E-08 & $1.33 \mathrm{E}-08$ & $1.13 \mathrm{E}-09$ \\
\hline SR 90 & $1.99 \mathrm{E}-10$ & $1.99 \mathrm{E}-10$ & $1.99 \mathrm{E}-10$ & $1.99 \mathrm{E}-10$ & $1.98 \mathrm{E}-10$ & $1.98 \mathrm{E}-10$ & $1.97 \mathrm{E}-10$ & $1.97 \mathrm{E}-10$ & $1.94 \mathrm{E}-10$ \\
\hline SR 91 & 4.79E-09 & $8.31 \mathrm{E}-10$ & $1.44 \mathrm{E}-10$ & $1.19 \mathrm{E}-16$ & $6.85 \mathrm{E}-32$ & $0.00 \mathrm{E}+00$ & $0.00 \mathrm{E}+00$ & $0.00 \mathrm{E}+00$ & $0.00 \mathrm{E}+00$ \\
\hline SR 93 & $8.84 \mathrm{E}-15$ & $0.00 \mathrm{E}+00$ & $0.00 \mathrm{E}+00$ & $0.00 \mathrm{E}+00$ & $0.00 \mathrm{E}+00$ & $0.00 \mathrm{E}+00$ & $0.00 \mathrm{E}+00$ & $0.00 \mathrm{E}+00$ & $0.00 \mathrm{E}+00$ \\
\hline Y 89M & $2.53 \mathrm{E}-03$ & $2.04 \mathrm{E}-03$ & $1.65 \mathrm{E}-03$ & $3.03 \mathrm{E}-04$ & $4.36 \mathrm{E}-06$ & $1.30 \mathrm{E}-11$ & $2.69 \mathrm{E}-15$ & $6.67 \mathrm{E}-20$ & $0.00 \mathrm{E}+00$ \\
\hline Y 90 & $1.19 \mathrm{E}-02$ & $9.17 \mathrm{E}-03$ & $7.07 \mathrm{E}-03$ & $8.84 \mathrm{E}-04$ & $4.88 \mathrm{E}-06$ & $1.99 \mathrm{E}-10$ & $1.97 \mathrm{E}-10$ & $1.97 \mathrm{E}-10$ & $1.94 \mathrm{E}-10$ \\
\hline Y 90M & $3.91 \mathrm{E}-09$ & $1.83 \mathrm{E}-11$ & $8.54 \mathrm{E}-14$ & $2.28 \mathrm{E}-32$ & $0.00 \mathrm{E}+00$ & $0.00 \mathrm{E}+00$ & $0.00 \mathrm{E}+00$ & $0.00 \mathrm{E}+00$ & $0.00 \mathrm{E}+00$ \\
\hline Y 91 & $1.15 \mathrm{E}-06$ & $1.14 \mathrm{E}-06$ & $1.13 \mathrm{E}-06$ & $1.03 \mathrm{E}-06$ & $8.09 \mathrm{E}-07$ & $3.97 \mathrm{E}-07$ & $2.47 \mathrm{E}-07$ & $1.37 \mathrm{E}-07$ & $1.62 \mathrm{E}-08$ \\
\hline Y 92 & $6.06 \mathrm{E}-07$ & $5.50 \mathrm{E}-09$ & $5.00 \mathrm{E}-11$ & $2.33 \mathrm{E}-27$ & $0.00 \mathrm{E}+00$ & $0.00 \mathrm{E}+00$ & $0.00 \mathrm{E}+00$ & $0.00 \mathrm{E}+00$ & $0.00 \mathrm{E}+00$ \\
\hline Y 93 & $8.84 \mathrm{E}-15$ & $1.71 \mathrm{E}-15$ & $3.39 \mathrm{E}-16$ & $6.43 \mathrm{E}-22$ & $0.00 \mathrm{E}+00$ & $0.00 \mathrm{E}+00$ & $0.00 \mathrm{E}+00$ & $0.00 \mathrm{E}+00$ & $0.00 \mathrm{E}+00$ \\
\hline Y 94 & $3.41 \mathrm{E}-09$ & $0.00 \mathrm{E}+00$ & $0.00 \mathrm{E}+00$ & $0.00 \mathrm{E}+00$ & $0.00 \mathrm{E}+00$ & $0.00 \mathrm{E}+00$ & $0.00 \mathrm{E}+00$ & $0.00 \mathrm{E}+00$ & $0.00 \mathrm{E}+00$ \\
\hline Y 96 & $1.18 \mathrm{E}-14$ & $0.00 \mathrm{E}+00$ & $0.00 \mathrm{E}+00$ & $0.00 \mathrm{E}+00$ & $0.00 \mathrm{E}+00$ & $0.00 \mathrm{E}+00$ & $0.00 \mathrm{E}+00$ & $0.00 \mathrm{E}+00$ & $0.00 \mathrm{E}+00$ \\
\hline ZR 89 & $2.53 \mathrm{E}-03$ & $2.05 \mathrm{E}-03$ & $1.66 \mathrm{E}-03$ & $3.03 \mathrm{E}-04$ & $4.36 \mathrm{E}-06$ & $1.30 \mathrm{E}-11$ & $2.69 \mathrm{E}-15$ & $6.68 \mathrm{E}-20$ & $0.00 \mathrm{E}+00$ \\
\hline ZR 93 & $3.12 \mathrm{E}-08$ & $3.12 \mathrm{E}-08$ & $3.12 \mathrm{E}-08$ & $3.12 \mathrm{E}-08$ & $3.12 \mathrm{E}-08$ & $3.12 \mathrm{E}-08$ & $3.12 \mathrm{E}-08$ & $3.12 \mathrm{E}-08$ & $3.12 \mathrm{E}-08$ \\
\hline ZR 95 & $5.85 \mathrm{E}-03$ & $5.78 \mathrm{E}-03$ & $5.72 \mathrm{E}-03$ & $5.25 \mathrm{E}-03$ & $4.22 \mathrm{E}-03$ & $2.21 \mathrm{E}-03$ & $1.43 \mathrm{E}-03$ & 8.32E-04 & $1.18 \mathrm{E}-04$ \\
\hline ZR 97 & $2.00 \mathrm{E}-04$ & $7.47 \mathrm{E}-05$ & $2.79 \mathrm{E}-05$ & $1.06 \mathrm{E}-08$ & $2.99 \mathrm{E}-17$ & $0.00 \mathrm{E}+00$ & $0.00 \mathrm{E}+00$ & $0.00 \mathrm{E}+00$ & $0.00 \mathrm{E}+00$ \\
\hline NB 92 & $4.96 \mathrm{E}-01$ & 4.64E-01 & 4.33E-01 & $2.51 \mathrm{E}-01$ & $6.41 \mathrm{E}-02$ & $1.07 \mathrm{E}-03$ & $6.98 \mathrm{E}-05$ & $2.30 \mathrm{E}-06$ & $1.07 \mathrm{E}-11$ \\
\hline NB 93M & $7.07 \mathrm{E}-10$ & $7.11 \mathrm{E}-10$ & $7.15 \mathrm{E}-10$ & $7.47 \mathrm{E}-10$ & $8.28 \mathrm{E}-10$ & $1.07 \mathrm{E}-09$ & $1.23 \mathrm{E}-09$ & $1.43 \mathrm{E}-09$ & $2.13 \mathrm{E}-09$ \\
\hline
\end{tabular}


BSU-8242 3 DPA As-Run Physics Analysis

\begin{tabular}{|c|c|c|c|c|c|c|c|c|c|}
\hline \multicolumn{10}{|c|}{ ACTIVATION PRODUCTS (Ci) } \\
\hline ISOTOPE & EOC & 1 Day & 2 Days & 10 Days & 30 Days & 90 Days & 130 Days & 180 Days & 360 Days \\
\hline NB 94 & 4.29E-03 & 4.29E-03 & 4.29E-03 & 4.29E-03 & 4.29E-03 & 4.29E-03 & 4.29E-03 & 4.29E-03 & 4.29E-03 \\
\hline NB 95 & $1.15 \mathrm{E}+01$ & $1.13 \mathrm{E}+01$ & $1.11 \mathrm{E}+01$ & $9.47 \mathrm{E}+00$ & $6.38 \mathrm{E}+00$ & $1.96 \mathrm{E}+00$ & $8.90 \mathrm{E}-01$ & $3.33 \mathrm{E}-01$ & $9.78 \mathrm{E}-03$ \\
\hline NB 95M & $4.16 \mathrm{E}-05$ & $4.14 \mathrm{E}-05$ & $4.12 \mathrm{E}-05$ & $3.87 \mathrm{E}-05$ & $3.13 \mathrm{E}-05$ & $1.64 \mathrm{E}-05$ & $1.06 \mathrm{E}-05$ & $6.17 \mathrm{E}-06$ & 8.78E-07 \\
\hline NB 96 & $6.23 \mathrm{E}-02$ & $3.05 \mathrm{E}-02$ & $1.50 \mathrm{E}-02$ & $5.01 \mathrm{E}-05$ & $3.25 \mathrm{E}-11$ & $8.85 \mathrm{E}-30$ & $0.00 \mathrm{E}+00$ & $0.00 \mathrm{E}+00$ & $0.00 \mathrm{E}+00$ \\
\hline NB 97 & $7.11 \mathrm{E}-03$ & $7.50 \mathrm{E}-05$ & $2.80 \mathrm{E}-05$ & $1.14 \mathrm{E}-08$ & $3.22 \mathrm{E}-17$ & $0.00 \mathrm{E}+00$ & $0.00 \mathrm{E}+00$ & $0.00 \mathrm{E}+00$ & $0.00 \mathrm{E}+00$ \\
\hline NB 97M & $1.89 \mathrm{E}-04$ & $7.07 \mathrm{E}-05$ & $2.64 \mathrm{E}-05$ & $1.01 \mathrm{E}-08$ & $2.83 \mathrm{E}-17$ & $0.00 \mathrm{E}+00$ & $0.00 \mathrm{E}+00$ & $0.00 \mathrm{E}+00$ & $0.00 \mathrm{E}+00$ \\
\hline NB 98 & $1.06 \mathrm{E}-03$ & $0.00 \mathrm{E}+00$ & $0.00 \mathrm{E}+00$ & $0.00 \mathrm{E}+00$ & $0.00 \mathrm{E}+00$ & $0.00 \mathrm{E}+00$ & $0.00 \mathrm{E}+00$ & $0.00 \mathrm{E}+00$ & $0.00 \mathrm{E}+00$ \\
\hline NB100 & $5.25 \mathrm{E}-05$ & $0.00 \mathrm{E}+00$ & $0.00 \mathrm{E}+00$ & $0.00 \mathrm{E}+00$ & $0.00 \mathrm{E}+00$ & $0.00 \mathrm{E}+00$ & $0.00 \mathrm{E}+00$ & $0.00 \mathrm{E}+00$ & $0.00 \mathrm{E}+00$ \\
\hline MO 93M & $1.46 \mathrm{E}-01$ & $1.29 \mathrm{E}-02$ & $1.14 \mathrm{E}-03$ & $4.15 \mathrm{E}-12$ & $0.00 \mathrm{E}+00$ & $0.00 \mathrm{E}+00$ & $0.00 \mathrm{E}+00$ & $0.00 \mathrm{E}+00$ & $0.00 \mathrm{E}+00$ \\
\hline MO 93 & $4.40 \mathrm{E}-04$ & $4.40 \mathrm{E}-04$ & $4.40 \mathrm{E}-04$ & $4.40 \mathrm{E}-04$ & $4.40 \mathrm{E}-04$ & $4.40 \mathrm{E}-04$ & 4.40E-04 & $4.40 \mathrm{E}-04$ & 4.40E-04 \\
\hline MO 99 & $1.02 \mathrm{E}+02$ & $7.95 \mathrm{E}+01$ & $6.18 \mathrm{E}+01$ & $8.23 \mathrm{E}+00$ & $5.32 \mathrm{E}-02$ & $1.44 \mathrm{E}-08$ & $6.02 \mathrm{E}-13$ & $2.02 \mathrm{E}-18$ & $0.00 \mathrm{E}+00$ \\
\hline MO101 & $2.54 \mathrm{E}+01$ & $0.00 \mathrm{E}+00$ & $0.00 \mathrm{E}+00$ & $0.00 \mathrm{E}+00$ & $0.00 \mathrm{E}+00$ & $0.00 \mathrm{E}+00$ & $0.00 \mathrm{E}+00$ & $0.00 \mathrm{E}+00$ & $0.00 \mathrm{E}+00$ \\
\hline TC 99 & $1.86 \mathrm{E}-05$ & $1.87 \mathrm{E}-05$ & $1.88 \mathrm{E}-05$ & $1.90 \mathrm{E}-05$ & $1.91 \mathrm{E}-05$ & $1.91 \mathrm{E}-05$ & $1.91 \mathrm{E}-05$ & $1.91 \mathrm{E}-05$ & $1.91 \mathrm{E}-05$ \\
\hline TC100 & $1.68 \mathrm{E}+00$ & $0.00 \mathrm{E}+00$ & $0.00 \mathrm{E}+00$ & $0.00 \mathrm{E}+00$ & $0.00 \mathrm{E}+00$ & $0.00 \mathrm{E}+00$ & $0.00 \mathrm{E}+00$ & $0.00 \mathrm{E}+00$ & $0.00 \mathrm{E}+00$ \\
\hline TC101 & $2.54 \mathrm{E}+01$ & $0.00 \mathrm{E}+00$ & $0.00 \mathrm{E}+00$ & $0.00 \mathrm{E}+00$ & $0.00 \mathrm{E}+00$ & $0.00 \mathrm{E}+00$ & $0.00 \mathrm{E}+00$ & $0.00 \mathrm{E}+00$ & $0.00 \mathrm{E}+00$ \\
\hline RU103 & $1.29 \mathrm{E}-03$ & $1.27 \mathrm{E}-03$ & $1.24 \mathrm{E}-03$ & $1.08 \mathrm{E}-03$ & $7.59 \mathrm{E}-04$ & $2.63 \mathrm{E}-04$ & $1.30 \mathrm{E}-04$ & $5.38 \mathrm{E}-05$ & $2.25 \mathrm{E}-06$ \\
\hline RU105 & $1.80 \mathrm{E}-08$ & $4.25 \mathrm{E}-10$ & $1.00 \mathrm{E}-11$ & $9.54 \mathrm{E}-25$ & $0.00 \mathrm{E}+00$ & $0.00 \mathrm{E}+00$ & $0.00 \mathrm{E}+00$ & $0.00 \mathrm{E}+00$ & $0.00 \mathrm{E}+00$ \\
\hline RU106 & $7.99 \mathrm{E}-15$ & $7.98 \mathrm{E}-15$ & $7.96 \mathrm{E}-15$ & $7.85 \mathrm{E}-15$ & $7.56 \mathrm{E}-15$ & $6.75 \mathrm{E}-15$ & $6.26 \mathrm{E}-15$ & $5.70 \mathrm{E}-15$ & $4.06 \mathrm{E}-15$ \\
\hline RH104 & $2.20 \mathrm{E}-05$ & $0.00 \mathrm{E}+00$ & $0.00 \mathrm{E}+00$ & $0.00 \mathrm{E}+00$ & $0.00 \mathrm{E}+00$ & $0.00 \mathrm{E}+00$ & $0.00 \mathrm{E}+00$ & $0.00 \mathrm{E}+00$ & $0.00 \mathrm{E}+00$ \\
\hline RH104M & $1.59 \mathrm{E}-06$ & $0.00 \mathrm{E}+00$ & $0.00 \mathrm{E}+00$ & $0.00 \mathrm{E}+00$ & $0.00 \mathrm{E}+00$ & $0.00 \mathrm{E}+00$ & $0.00 \mathrm{E}+00$ & $0.00 \mathrm{E}+00$ & $0.00 \mathrm{E}+00$ \\
\hline RH105 & $1.41 \mathrm{E}-08$ & $1.04 \mathrm{E}-08$ & $6.50 \mathrm{E}-09$ & $1.51 \mathrm{E}-10$ & $1.24 \mathrm{E}-14$ & $6.82 \mathrm{E}-27$ & $0.00 \mathrm{E}+00$ & $0.00 \mathrm{E}+00$ & $0.00 \mathrm{E}+00$ \\
\hline RH105M & $5.05 \mathrm{E}-09$ & $1.19 \mathrm{E}-10$ & $2.81 \mathrm{E}-12$ & $2.68 \mathrm{E}-25$ & $0.00 \mathrm{E}+00$ & $0.00 \mathrm{E}+00$ & $0.00 \mathrm{E}+00$ & $0.00 \mathrm{E}+00$ & $0.00 \mathrm{E}+00$ \\
\hline RH106 & 2.69E-09 & $7.98 \mathrm{E}-15$ & $7.96 \mathrm{E}-15$ & $7.85 \mathrm{E}-15$ & $7.56 \mathrm{E}-15$ & $6.75 \mathrm{E}-15$ & $6.26 \mathrm{E}-15$ & $5.70 \mathrm{E}-15$ & $4.06 \mathrm{E}-15$ \\
\hline RH106M & $1.30 \mathrm{E}-09$ & $6.78 \mathrm{E}-13$ & $3.53 \mathrm{E}-16$ & $0.00 \mathrm{E}+00$ & $0.00 \mathrm{E}+00$ & $0.00 \mathrm{E}+00$ & $0.00 \mathrm{E}+00$ & $0.00 \mathrm{E}+00$ & $0.00 \mathrm{E}+00$ \\
\hline PD107M & $1.05 \mathrm{E}-13$ & $0.00 \mathrm{E}+00$ & $0.00 \mathrm{E}+00$ & $0.00 \mathrm{E}+00$ & $0.00 \mathrm{E}+00$ & $0.00 \mathrm{E}+00$ & $0.00 \mathrm{E}+00$ & $0.00 \mathrm{E}+00$ & $0.00 \mathrm{E}+00$ \\
\hline PD109 & $5.10 \mathrm{E}-16$ & $1.58 \mathrm{E}-16$ & $3.38 \mathrm{E}-17$ & $1.72 \mathrm{E}-21$ & $2.28 \mathrm{E}-32$ & $0.00 \mathrm{E}+00$ & $0.00 \mathrm{E}+00$ & $0.00 \mathrm{E}+00$ & $0.00 \mathrm{E}+00$ \\
\hline
\end{tabular}


BSU-8242 3 DPA As-Run Physics Analysis

\begin{tabular}{|c|c|c|c|c|c|c|c|c|c|}
\hline \multicolumn{10}{|c|}{ ACTIVATION PRODUCTS (Ci) } \\
\hline ISOTOPE & EOC & 1 Day & 2 Days & 10 Days & 30 Days & 90 Days & 130 Days & 180 Days & 360 Days \\
\hline AG109M & $5.10 \mathrm{E}-16$ & $1.58 \mathrm{E}-16$ & $4.60 \mathrm{E}-17$ & $1.72 \mathrm{E}-21$ & $2.28 \mathrm{E}-32$ & $0.00 \mathrm{E}+00$ & $0.00 \mathrm{E}+00$ & $0.00 \mathrm{E}+00$ & $0.00 \mathrm{E}+00$ \\
\hline TM172 & $1.92 \mathrm{E}-09$ & $1.48 \mathrm{E}-09$ & $1.14 \mathrm{E}-09$ & $1.40 \mathrm{E}-10$ & $7.50 \mathrm{E}-13$ & $1.15 \mathrm{E}-19$ & $3.28 \mathrm{E}-24$ & $6.85 \mathrm{E}-30$ & $0.00 \mathrm{E}+00$ \\
\hline TM173 & $1.71 \mathrm{E}-09$ & $2.27 \mathrm{E}-10$ & $3.02 \mathrm{E}-11$ & $2.92 \mathrm{E}-18$ & $0.00 \mathrm{E}+00$ & $0.00 \mathrm{E}+00$ & $0.00 \mathrm{E}+00$ & $0.00 \mathrm{E}+00$ & $0.00 \mathrm{E}+00$ \\
\hline YB175 & $2.90 \mathrm{E}-12$ & $2.46 \mathrm{E}-12$ & $2.09 \mathrm{E}-12$ & $5.55 \mathrm{E}-13$ & $2.03 \mathrm{E}-14$ & $9.92 \mathrm{E}-19$ & $1.09 \mathrm{E}-18$ & $2.79 \mathrm{E}-22$ & $0.00 \mathrm{E}+00$ \\
\hline YB175M & $2.05 \mathrm{E}-12$ & $0.00 \mathrm{E}+00$ & $0.00 \mathrm{E}+00$ & $0.00 \mathrm{E}+00$ & $0.00 \mathrm{E}+00$ & $0.00 \mathrm{E}+00$ & $0.00 \mathrm{E}+00$ & $0.00 \mathrm{E}+00$ & $0.00 \mathrm{E}+00$ \\
\hline LU176 & $6.68 \mathrm{E}-15$ & $6.68 \mathrm{E}-15$ & $6.68 \mathrm{E}-15$ & $6.68 \mathrm{E}-15$ & $6.68 \mathrm{E}-15$ & $6.68 \mathrm{E}-15$ & $6.68 \mathrm{E}-15$ & $6.68 \mathrm{E}-15$ & $6.68 \mathrm{E}-15$ \\
\hline LU176M & $5.69 \mathrm{E}-03$ & $6.26 \mathrm{E}-05$ & $6.90 \mathrm{E}-07$ & $1.50 \mathrm{E}-22$ & $0.00 \mathrm{E}+00$ & $0.00 \mathrm{E}+00$ & $0.00 \mathrm{E}+00$ & $0.00 \mathrm{E}+00$ & $0.00 \mathrm{E}+00$ \\
\hline LU177 & $1.74 \mathrm{E}-03$ & $1.57 \mathrm{E}-03$ & $1.41 \mathrm{E}-03$ & $6.18 \mathrm{E}-04$ & $7.86 \mathrm{E}-05$ & $3.98 \mathrm{E}-07$ & $2.02 \mathrm{E}-07$ & $1.60 \mathrm{E}-07$ & $7.14 \mathrm{E}-08$ \\
\hline LU177M & $1.55 \mathrm{E}-06$ & $1.55 \mathrm{E}-06$ & $1.54 \mathrm{E}-06$ & $1.48 \mathrm{E}-06$ & $1.36 \mathrm{E}-06$ & $1.04 \mathrm{E}-06$ & $8.68 \mathrm{E}-07$ & $6.94 \mathrm{E}-07$ & $3.10 \mathrm{E}-07$ \\
\hline HF175 & $2.02 \mathrm{E}-02$ & $2.00 \mathrm{E}-02$ & $1.98 \mathrm{E}-02$ & $1.83 \mathrm{E}-02$ & $1.50 \mathrm{E}-02$ & $8.30 \mathrm{E}-03$ & $5.58 \mathrm{E}-03$ & $3.40 \mathrm{E}-03$ & $5.72 \mathrm{E}-04$ \\
\hline HF178M & $4.99 \mathrm{E}-03$ & $0.00 \mathrm{E}+00$ & $0.00 \mathrm{E}+00$ & $0.00 \mathrm{E}+00$ & $0.00 \mathrm{E}+00$ & $0.00 \mathrm{E}+00$ & $0.00 \mathrm{E}+00$ & $0.00 \mathrm{E}+00$ & $0.00 \mathrm{E}+00$ \\
\hline HF179M & $5.49 \mathrm{E}+00$ & $0.00 \mathrm{E}+00$ & $0.00 \mathrm{E}+00$ & $0.00 \mathrm{E}+00$ & $0.00 \mathrm{E}+00$ & $0.00 \mathrm{E}+00$ & $0.00 \mathrm{E}+00$ & $0.00 \mathrm{E}+00$ & $0.00 \mathrm{E}+00$ \\
\hline HF180M & $3.26 \mathrm{E}-02$ & $1.59 \mathrm{E}-03$ & $7.70 \mathrm{E}-05$ & $2.39 \mathrm{E}-15$ & $0.00 \mathrm{E}+00$ & $0.00 \mathrm{E}+00$ & $0.00 \mathrm{E}+00$ & $0.00 \mathrm{E}+00$ & $0.00 \mathrm{E}+00$ \\
\hline HF181 & 4.64E-01 & $4.56 \mathrm{E}-01$ & $4.49 \mathrm{E}-01$ & $3.94 \mathrm{E}-01$ & $2.84 \mathrm{E}-01$ & $1.07 \mathrm{E}-01$ & $5.54 \mathrm{E}-02$ & $2.45 \mathrm{E}-02$ & $1.29 \mathrm{E}-03$ \\
\hline HF182 & $1.93 \mathrm{E}-10$ & $1.93 \mathrm{E}-10$ & $1.93 \mathrm{E}-10$ & $1.93 \mathrm{E}-10$ & $1.93 \mathrm{E}-10$ & $1.93 \mathrm{E}-10$ & $1.93 \mathrm{E}-10$ & $1.93 \mathrm{E}-10$ & $1.93 \mathrm{E}-10$ \\
\hline TA180 & $1.32 \mathrm{E}-14$ & $1.32 \mathrm{E}-14$ & $1.32 \mathrm{E}-14$ & $1.32 \mathrm{E}-14$ & $1.32 \mathrm{E}-14$ & $1.32 \mathrm{E}-14$ & $1.32 \mathrm{E}-14$ & $1.32 \mathrm{E}-14$ & $1.32 \mathrm{E}-14$ \\
\hline TA182 & $3.33 \mathrm{E}+02$ & $3.31 \mathrm{E}+02$ & $3.29 \mathrm{E}+02$ & $3.13 \mathrm{E}+02$ & $2.78 \mathrm{E}+02$ & $1.93 \mathrm{E}+02$ & $1.52 \mathrm{E}+02$ & $1.12 \mathrm{E}+02$ & $3.80 \mathrm{E}+01$ \\
\hline TA182M & $1.76 \mathrm{E}+00$ & $0.00 \mathrm{E}+00$ & $0.00 \mathrm{E}+00$ & $0.00 \mathrm{E}+00$ & $0.00 \mathrm{E}+00$ & $0.00 \mathrm{E}+00$ & $0.00 \mathrm{E}+00$ & $0.00 \mathrm{E}+00$ & $0.00 \mathrm{E}+00$ \\
\hline TA183 & $3.39 \mathrm{E}+03$ & $2.96 \mathrm{E}+03$ & $2.59 \mathrm{E}+03$ & $8.72 \mathrm{E}+02$ & $5.75 \mathrm{E}+01$ & $1.65 \mathrm{E}-02$ & $7.20 \mathrm{E}-05$ & $8.05 \mathrm{E}-08$ & $1.91 \mathrm{E}-18$ \\
\hline W181 & $2.02 \mathrm{E}-15$ & $2.01 \mathrm{E}-15$ & $2.00 \mathrm{E}-15$ & $1.91 \mathrm{E}-15$ & $1.70 \mathrm{E}-15$ & $1.21 \mathrm{E}-15$ & $9.62 \mathrm{E}-16$ & $7.23 \mathrm{E}-16$ & $2.58 \mathrm{E}-16$ \\
\hline $\mathrm{W} 183 \mathrm{M}$ & $4.08 \mathrm{E}-01$ & $0.00 \mathrm{E}+00$ & $0.00 \mathrm{E}+00$ & $0.00 \mathrm{E}+00$ & $0.00 \mathrm{E}+00$ & $0.00 \mathrm{E}+00$ & $0.00 \mathrm{E}+00$ & $0.00 \mathrm{E}+00$ & $0.00 \mathrm{E}+00$ \\
\hline W185 & $6.62 \mathrm{E}-01$ & $6.56 \mathrm{E}-01$ & $6.50 \mathrm{E}-01$ & $6.04 \mathrm{E}-01$ & $5.02 \mathrm{E}-01$ & $2.89 \mathrm{E}-01$ & $2.00 \mathrm{E}-01$ & $1.26 \mathrm{E}-01$ & $2.39 \mathrm{E}-02$ \\
\hline $\mathrm{W} 185 \mathrm{M}$ & $2.06 \mathrm{E}-03$ & $0.00 \mathrm{E}+00$ & $0.00 \mathrm{E}+00$ & $0.00 \mathrm{E}+00$ & $0.00 \mathrm{E}+00$ & $0.00 \mathrm{E}+00$ & $0.00 \mathrm{E}+00$ & $0.00 \mathrm{E}+00$ & $0.00 \mathrm{E}+00$ \\
\hline W187 & $4.82 \mathrm{E}-04$ & $2.40 \mathrm{E}-04$ & $1.20 \mathrm{E}-04$ & $4.58 \mathrm{E}-07$ & $4.12 \mathrm{E}-13$ & $2.97 \mathrm{E}-31$ & $0.00 \mathrm{E}+00$ & $0.00 \mathrm{E}+00$ & $0.00 \mathrm{E}+00$ \\
\hline W188 & $1.18 \mathrm{E}-06$ & $1.17 \mathrm{E}-06$ & $1.16 \mathrm{E}-06$ & $1.07 \mathrm{E}-06$ & $8.76 \mathrm{E}-07$ & $4.81 \mathrm{E}-07$ & $3.23 \mathrm{E}-07$ & $1.96 \mathrm{E}-07$ & $3.25 \mathrm{E}-08$ \\
\hline RE186 & $1.37 \mathrm{E}-01$ & $1.14 \mathrm{E}-01$ & $9.48 \mathrm{E}-02$ & $2.18 \mathrm{E}-02$ & $5.56 \mathrm{E}-04$ & $9.17 \mathrm{E}-09$ & $5.95 \mathrm{E}-12$ & $6.15 \mathrm{E}-16$ & $2.76 \mathrm{E}-30$ \\
\hline
\end{tabular}




\begin{tabular}{|lrr|}
\hline TEM-10200-1, Rev. 11 & ENGINEERING CALCULATIONS AND ANALYSIS & ECAR- 4951, Rev. 1 \\
$11 / 20 / 2019$ & Page 51 of 104 \\
& BSU-8242 3 DPA As-Run Physics Analysis & \\
\hline
\end{tabular}

\begin{tabular}{|l|c|c|c|c|c|c|c|c|c|}
\hline \multicolumn{10}{|c|}{ ACTIVATION PRODUCTS (Ci) } \\
\hline ISOTOPE & EOC & 1 Day & 2 Days & 10 Days & 30 Days & 90 Days & 130 Days & 180 Days & 360 Days \\
\hline RE187 & $6.26 \mathrm{E}-16$ & $6.39 \mathrm{E}-16$ & $6.46 \mathrm{E}-16$ & $6.52 \mathrm{E}-16$ & $6.52 \mathrm{E}-16$ & $6.52 \mathrm{E}-16$ & $6.52 \mathrm{E}-16$ & $6.52 \mathrm{E}-16$ & $6.52 \mathrm{E}-16$ \\
\hline RE188 & $2.06 \mathrm{E}-05$ & $8.60 \mathrm{E}-06$ & $3.96 \mathrm{E}-06$ & $1.08 \mathrm{E}-06$ & $8.85 \mathrm{E}-07$ & $4.86 \mathrm{E}-07$ & $3.26 \mathrm{E}-07$ & $1.98 \mathrm{E}-07$ & $3.28 \mathrm{E}-08$ \\
\hline RE188M & $1.90 \mathrm{E}-05$ & $0.00 \mathrm{E}+00$ & $0.00 \mathrm{E}+00$ & $0.00 \mathrm{E}+00$ & $0.00 \mathrm{E}+00$ & $0.00 \mathrm{E}+00$ & $0.00 \mathrm{E}+00$ & $0.00 \mathrm{E}+00$ & $0.00 \mathrm{E}+00$ \\
\hline RE189 & $3.41 \mathrm{E}-10$ & $1.72 \mathrm{E}-10$ & $8.67 \mathrm{E}-11$ & $3.62 \mathrm{E}-13$ & $4.10 \mathrm{E}-19$ & $0.00 \mathrm{E}+00$ & $0.00 \mathrm{E}+00$ & $0.00 \mathrm{E}+00$ & $0.00 \mathrm{E}+00$ \\
\hline OS190M & $9.17 \mathrm{E}-14$ & $0.00 \mathrm{E}+00$ & $0.00 \mathrm{E}+00$ & $0.00 \mathrm{E}+00$ & $0.00 \mathrm{E}+00$ & $0.00 \mathrm{E}+00$ & $0.00 \mathrm{E}+00$ & $0.00 \mathrm{E}+00$ & $0.00 \mathrm{E}+00$ \\
\hline OS191 & $1.34 \mathrm{E}-11$ & $1.33 \mathrm{E}-11$ & $1.28 \mathrm{E}-11$ & $8.97 \mathrm{E}-12$ & $3.65 \mathrm{E}-12$ & $2.45 \mathrm{E}-13$ & $4.06 \mathrm{E}-14$ & $4.28 \mathrm{E}-15$ & $1.30 \mathrm{E}-18$ \\
\hline OS191M & $1.98 \mathrm{E}-11$ & $5.50 \mathrm{E}-12$ & $1.53 \mathrm{E}-12$ & $5.47 \mathrm{E}-17$ & $4.20 \mathrm{E}-28$ & $0.00 \mathrm{E}+00$ & $0.00 \mathrm{E}+00$ & $0.00 \mathrm{E}+00$ & $0.00 \mathrm{E}+00$ \\
\hline IR192 & $3.42 \mathrm{E}-13$ & $3.39 \mathrm{E}-13$ & $3.36 \mathrm{E}-13$ & $3.11 \mathrm{E}-13$ & $2.58 \mathrm{E}-13$ & $1.47 \mathrm{E}-13$ & $1.01 \mathrm{E}-13$ & $6.34 \mathrm{E}-14$ & $1.18 \mathrm{E}-14$ \\
\hline IR194 & $1.71 \mathrm{E}-14$ & $7.18 \mathrm{E}-15$ & $3.01 \mathrm{E}-15$ & $2.89 \mathrm{E}-18$ & $8.22 \mathrm{E}-26$ & $0.00 \mathrm{E}+00$ & $0.00 \mathrm{E}+00$ & $0.00 \mathrm{E}+00$ & $0.00 \mathrm{E}+00$ \\
\hline IR194M & $9.03 \mathrm{E}-16$ & $0.00 \mathrm{E}+00$ & $0.00 \mathrm{E}+00$ & $0.00 \mathrm{E}+00$ & $0.00 \mathrm{E}+00$ & $0.00 \mathrm{E}+00$ & $0.00 \mathrm{E}+00$ & $0.00 \mathrm{E}+00$ & $0.00 \mathrm{E}+00$ \\
\hline PT193M & $2.25 \mathrm{E}-16$ & $1.89 \mathrm{E}-16$ & $1.58 \mathrm{E}-16$ & $4.27 \mathrm{E}-17$ & $6.01 \mathrm{E}-19$ & $3.79 \mathrm{E}-23$ & $3.79 \mathrm{E}-23$ & $1.20 \mathrm{E}-26$ & $0.00 \mathrm{E}+00$ \\
\hline TOTAL & $1.05 \mathrm{E}+04$ & $6.10 \mathrm{E}+03$ & $5.63 \mathrm{E}+03$ & $3.43 \mathrm{E}+03$ & $1.84 \mathrm{E}+03$ & $7.89 \mathrm{E}+02$ & $5.60 \mathrm{E}+02$ & $4.26 \mathrm{E}+02$ & $2.70 \mathrm{E}+02$ \\
\hline
\end{tabular}


BSU-8242 3 DPA As-Run Physics Analysis

Table 20. Source Terms for 'A7 400C 3 DPA Y' Capsule.

\begin{tabular}{|c|c|c|c|c|c|c|c|c|c|}
\hline \multicolumn{10}{|c|}{ ACTIVATION PRODUCTS (Ci) } \\
\hline ISOTOPE & EOC & 1 Day & 2 Days & 10 Days & 30 Days & 90 Days & 130 Days & 180 Days & 360 Days \\
\hline $\mathrm{H} 3$ & $1.03 \mathrm{E}-04$ & $1.03 \mathrm{E}-04$ & $1.03 \mathrm{E}-04$ & $1.03 \mathrm{E}-04$ & $1.03 \mathrm{E}-04$ & $1.02 \mathrm{E}-04$ & $1.01 \mathrm{E}-04$ & $1.00 \mathrm{E}-04$ & $9.76 \mathrm{E}-05$ \\
\hline $\mathrm{H} 4$ & $3.37 \mathrm{E}-11$ & $0.00 \mathrm{E}+00$ & $0.00 \mathrm{E}+00$ & $0.00 \mathrm{E}+00$ & $0.00 \mathrm{E}+00$ & $0.00 \mathrm{E}+00$ & $0.00 \mathrm{E}+00$ & $0.00 \mathrm{E}+00$ & $0.00 \mathrm{E}+00$ \\
\hline HE 6 & $1.61 \mathrm{E}-04$ & $0.00 \mathrm{E}+00$ & $0.00 \mathrm{E}+00$ & $0.00 \mathrm{E}+00$ & $0.00 \mathrm{E}+00$ & $0.00 \mathrm{E}+00$ & $0.00 \mathrm{E}+00$ & $0.00 \mathrm{E}+00$ & $0.00 \mathrm{E}+00$ \\
\hline LI 8 & $3.19 \mathrm{E}-07$ & $0.00 \mathrm{E}+00$ & $0.00 \mathrm{E}+00$ & $0.00 \mathrm{E}+00$ & $0.00 \mathrm{E}+00$ & $0.00 \mathrm{E}+00$ & $0.00 \mathrm{E}+00$ & $0.00 \mathrm{E}+00$ & $0.00 \mathrm{E}+00$ \\
\hline BE 8 & $2.77 \mathrm{E}-04$ & $0.00 \mathrm{E}+00$ & $0.00 \mathrm{E}+00$ & $0.00 \mathrm{E}+00$ & $0.00 \mathrm{E}+00$ & $0.00 \mathrm{E}+00$ & $0.00 \mathrm{E}+00$ & $0.00 \mathrm{E}+00$ & $0.00 \mathrm{E}+00$ \\
\hline BE 10 & $1.42 \mathrm{E}-09$ & $1.42 \mathrm{E}-09$ & $1.42 \mathrm{E}-09$ & $1.42 \mathrm{E}-09$ & $1.42 \mathrm{E}-09$ & $1.42 \mathrm{E}-09$ & $1.42 \mathrm{E}-09$ & $1.42 \mathrm{E}-09$ & $1.42 \mathrm{E}-09$ \\
\hline BE 11 & 8.89E-09 & $0.00 \mathrm{E}+00$ & $0.00 \mathrm{E}+00$ & $0.00 \mathrm{E}+00$ & $0.00 \mathrm{E}+00$ & $0.00 \mathrm{E}+00$ & $0.00 \mathrm{E}+00$ & $0.00 \mathrm{E}+00$ & $0.00 \mathrm{E}+00$ \\
\hline B 12 & $6.65 \mathrm{E}-05$ & $0.00 \mathrm{E}+00$ & $0.00 \mathrm{E}+00$ & $0.00 \mathrm{E}+00$ & $0.00 \mathrm{E}+00$ & $0.00 \mathrm{E}+00$ & $0.00 \mathrm{E}+00$ & $0.00 \mathrm{E}+00$ & $0.00 \mathrm{E}+00$ \\
\hline C 14 & $3.28 \mathrm{E}-03$ & $3.28 \mathrm{E}-03$ & $3.28 \mathrm{E}-03$ & $3.28 \mathrm{E}-03$ & $3.28 \mathrm{E}-03$ & $3.28 \mathrm{E}-03$ & $3.28 \mathrm{E}-03$ & $3.28 \mathrm{E}-03$ & $3.28 \mathrm{E}-03$ \\
\hline C 15 & $7.59 \mathrm{E}-07$ & $0.00 \mathrm{E}+00$ & $0.00 \mathrm{E}+00$ & $0.00 \mathrm{E}+00$ & $0.00 \mathrm{E}+00$ & $0.00 \mathrm{E}+00$ & $0.00 \mathrm{E}+00$ & $0.00 \mathrm{E}+00$ & $0.00 \mathrm{E}+00$ \\
\hline N 16 & $2.53 \mathrm{E}-06$ & $0.00 \mathrm{E}+00$ & $0.00 \mathrm{E}+00$ & $0.00 \mathrm{E}+00$ & $0.00 \mathrm{E}+00$ & $0.00 \mathrm{E}+00$ & $0.00 \mathrm{E}+00$ & $0.00 \mathrm{E}+00$ & $0.00 \mathrm{E}+00$ \\
\hline O 19 & $1.05 \mathrm{E}-10$ & $0.00 \mathrm{E}+00$ & $0.00 \mathrm{E}+00$ & $0.00 \mathrm{E}+00$ & $0.00 \mathrm{E}+00$ & $0.00 \mathrm{E}+00$ & $0.00 \mathrm{E}+00$ & $0.00 \mathrm{E}+00$ & $0.00 \mathrm{E}+00$ \\
\hline F 20 & $1.52 \mathrm{E}-07$ & $0.00 \mathrm{E}+00$ & $0.00 \mathrm{E}+00$ & $0.00 \mathrm{E}+00$ & $0.00 \mathrm{E}+00$ & $0.00 \mathrm{E}+00$ & $0.00 \mathrm{E}+00$ & $0.00 \mathrm{E}+00$ & $0.00 \mathrm{E}+00$ \\
\hline NE 23 & $6.09 \mathrm{E}-02$ & $0.00 \mathrm{E}+00$ & $0.00 \mathrm{E}+00$ & $0.00 \mathrm{E}+00$ & $0.00 \mathrm{E}+00$ & $0.00 \mathrm{E}+00$ & $0.00 \mathrm{E}+00$ & $0.00 \mathrm{E}+00$ & $0.00 \mathrm{E}+00$ \\
\hline NA 24 & $1.50 \mathrm{E}+01$ & $4.94 \mathrm{E}+00$ & $1.63 \mathrm{E}+00$ & $2.28 \mathrm{E}-04$ & $5.31 \mathrm{E}-14$ & $0.00 \mathrm{E}+00$ & $0.00 \mathrm{E}+00$ & $0.00 \mathrm{E}+00$ & $0.00 \mathrm{E}+00$ \\
\hline NA $24 \mathrm{M}$ & $3.37 \mathrm{E}-05$ & $0.00 \mathrm{E}+00$ & $0.00 \mathrm{E}+00$ & $0.00 \mathrm{E}+00$ & $0.00 \mathrm{E}+00$ & $0.00 \mathrm{E}+00$ & $0.00 \mathrm{E}+00$ & $0.00 \mathrm{E}+00$ & $0.00 \mathrm{E}+00$ \\
\hline NA 25 & $3.68 \mathrm{E}-02$ & $0.00 \mathrm{E}+00$ & $0.00 \mathrm{E}+00$ & $0.00 \mathrm{E}+00$ & $0.00 \mathrm{E}+00$ & $0.00 \mathrm{E}+00$ & $0.00 \mathrm{E}+00$ & $0.00 \mathrm{E}+00$ & $0.00 \mathrm{E}+00$ \\
\hline MG 27 & $7.09 \mathrm{E}+01$ & $0.00 \mathrm{E}+00$ & $0.00 \mathrm{E}+00$ & $0.00 \mathrm{E}+00$ & $0.00 \mathrm{E}+00$ & $0.00 \mathrm{E}+00$ & $0.00 \mathrm{E}+00$ & $0.00 \mathrm{E}+00$ & $0.00 \mathrm{E}+00$ \\
\hline MG 28 & $1.99 \mathrm{E}-07$ & 8.98E-08 & 4.05E-08 & $6.98 \mathrm{E}-11$ & 8.59E-18 & $0.00 \mathrm{E}+00$ & $0.00 \mathrm{E}+00$ & $0.00 \mathrm{E}+00$ & $0.00 \mathrm{E}+00$ \\
\hline AL 28 & $1.69 \mathrm{E}+03$ & $9.00 \mathrm{E}-08$ & $4.06 \mathrm{E}-08$ & $7.00 \mathrm{E}-11$ & $8.61 \mathrm{E}-18$ & $0.00 \mathrm{E}+00$ & $0.00 \mathrm{E}+00$ & $0.00 \mathrm{E}+00$ & $0.00 \mathrm{E}+00$ \\
\hline AL 29 & 4.27E-02 & $0.00 \mathrm{E}+00$ & $0.00 \mathrm{E}+00$ & $0.00 \mathrm{E}+00$ & $0.00 \mathrm{E}+00$ & $0.00 \mathrm{E}+00$ & $0.00 \mathrm{E}+00$ & $0.00 \mathrm{E}+00$ & $0.00 \mathrm{E}+00$ \\
\hline AL 30 & 4.12E-05 & $0.00 \mathrm{E}+00$ & $0.00 \mathrm{E}+00$ & $0.00 \mathrm{E}+00$ & $0.00 \mathrm{E}+00$ & $0.00 \mathrm{E}+00$ & $0.00 \mathrm{E}+00$ & $0.00 \mathrm{E}+00$ & $0.00 \mathrm{E}+00$ \\
\hline SI 31 & $6.49 \mathrm{E}-01$ & $1.14 \mathrm{E}-03$ & $2.00 \mathrm{E}-06$ & $0.00 \mathrm{E}+00$ & $0.00 \mathrm{E}+00$ & $0.00 \mathrm{E}+00$ & $0.00 \mathrm{E}+00$ & $0.00 \mathrm{E}+00$ & $0.00 \mathrm{E}+00$ \\
\hline SI 32 & $1.84 \mathrm{E}-10$ & $1.84 \mathrm{E}-10$ & $1.84 \mathrm{E}-10$ & $1.84 \mathrm{E}-10$ & $1.84 \mathrm{E}-10$ & $1.84 \mathrm{E}-10$ & $1.84 \mathrm{E}-10$ & $1.84 \mathrm{E}-10$ & $1.84 \mathrm{E}-10$ \\
\hline P 32 & $7.48 \mathrm{E}-01$ & $7.13 \mathrm{E}-01$ & $6.79 \mathrm{E}-01$ & 4.61E-01 & $1.75 \mathrm{E}-01$ & $9.53 \mathrm{E}-03$ & $1.37 \mathrm{E}-03$ & $1.22 \mathrm{E}-04$ & $1.99 \mathrm{E}-08$ \\
\hline
\end{tabular}


BSU-8242 3 DPA As-Run Physics Analysis

\begin{tabular}{|c|c|c|c|c|c|c|c|c|c|}
\hline \multicolumn{10}{|c|}{ ACTIVATION PRODUCTS (Ci) } \\
\hline ISOTOPE & EOC & 1 Day & 2 Days & 10 Days & 30 Days & 90 Days & 130 Days & 180 Days & 360 Days \\
\hline P 33 & $2.38 \mathrm{E}-05$ & $2.31 \mathrm{E}-05$ & $2.25 \mathrm{E}-05$ & $1.80 \mathrm{E}-05$ & $1.04 \mathrm{E}-05$ & $1.96 \mathrm{E}-06$ & $6.47 \mathrm{E}-07$ & $1.62 \mathrm{E}-07$ & $1.10 \mathrm{E}-09$ \\
\hline P 34 & $1.49 \mathrm{E}-04$ & $0.00 \mathrm{E}+00$ & $0.00 \mathrm{E}+00$ & $0.00 \mathrm{E}+00$ & $0.00 \mathrm{E}+00$ & $0.00 \mathrm{E}+00$ & $0.00 \mathrm{E}+00$ & $0.00 \mathrm{E}+00$ & $0.00 \mathrm{E}+00$ \\
\hline S 35 & $1.00 \mathrm{E}-02$ & 9.92E-03 & $9.84 \mathrm{E}-03$ & $9.24 \mathrm{E}-03$ & $7.90 \mathrm{E}-03$ & $4.92 \mathrm{E}-03$ & $3.59 \mathrm{E}-03$ & $2.42 \mathrm{E}-03$ & $5.87 \mathrm{E}-04$ \\
\hline S 37 & $4.29 \mathrm{E}-05$ & $0.00 \mathrm{E}+00$ & $0.00 \mathrm{E}+00$ & $0.00 \mathrm{E}+00$ & $0.00 \mathrm{E}+00$ & $0.00 \mathrm{E}+00$ & $0.00 \mathrm{E}+00$ & $0.00 \mathrm{E}+00$ & $0.00 \mathrm{E}+00$ \\
\hline CL 36 & $2.99 \mathrm{E}-10$ & $2.99 \mathrm{E}-10$ & $2.99 \mathrm{E}-10$ & $2.99 \mathrm{E}-10$ & $2.99 \mathrm{E}-10$ & $2.99 \mathrm{E}-10$ & $2.99 \mathrm{E}-10$ & $2.99 \mathrm{E}-10$ & $2.99 \mathrm{E}-10$ \\
\hline CL 38 & $2.70 \mathrm{E}-08$ & $6.08 \mathrm{E}-20$ & $1.37 \mathrm{E}-31$ & $0.00 \mathrm{E}+00$ & $0.00 \mathrm{E}+00$ & $0.00 \mathrm{E}+00$ & $0.00 \mathrm{E}+00$ & $0.00 \mathrm{E}+00$ & $0.00 \mathrm{E}+00$ \\
\hline CL 38M & $3.21 \mathrm{E}-10$ & $0.00 \mathrm{E}+00$ & $0.00 \mathrm{E}+00$ & $0.00 \mathrm{E}+00$ & $0.00 \mathrm{E}+00$ & $0.00 \mathrm{E}+00$ & $0.00 \mathrm{E}+00$ & $0.00 \mathrm{E}+00$ & $0.00 \mathrm{E}+00$ \\
\hline AR 37 & $4.28 \mathrm{E}-13$ & $4.20 \mathrm{E}-13$ & 4.12E-13 & $3.51 \mathrm{E}-13$ & $2.37 \mathrm{E}-13$ & $7.21 \mathrm{E}-14$ & $3.27 \mathrm{E}-14$ & $1.22 \mathrm{E}-14$ & $3.45 \mathrm{E}-16$ \\
\hline AR 39 & $5.03 \mathrm{E}-15$ & $5.03 \mathrm{E}-15$ & $5.03 \mathrm{E}-15$ & $5.03 \mathrm{E}-15$ & $5.03 \mathrm{E}-15$ & $5.03 \mathrm{E}-15$ & $5.03 \mathrm{E}-15$ & $5.03 \mathrm{E}-15$ & $5.02 \mathrm{E}-15$ \\
\hline AR 41 & $5.81 \mathrm{E}-13$ & $6.45 \mathrm{E}-17$ & $7.16 \mathrm{E}-21$ & $0.00 \mathrm{E}+00$ & $0.00 \mathrm{E}+00$ & $0.00 \mathrm{E}+00$ & $0.00 \mathrm{E}+00$ & $0.00 \mathrm{E}+00$ & $0.00 \mathrm{E}+00$ \\
\hline K 42 & $4.87 \mathrm{E}-16$ & $1.41 \mathrm{E}-16$ & $2.17 \mathrm{E}-17$ & $4.58 \mathrm{E}-22$ & $0.00 \mathrm{E}+00$ & $0.00 \mathrm{E}+00$ & $0.00 \mathrm{E}+00$ & $0.00 \mathrm{E}+00$ & $0.00 \mathrm{E}+00$ \\
\hline K 44 & $1.33 \mathrm{E}-12$ & $2.28 \mathrm{E}-32$ & $0.00 \mathrm{E}+00$ & $0.00 \mathrm{E}+00$ & $0.00 \mathrm{E}+00$ & $0.00 \mathrm{E}+00$ & $0.00 \mathrm{E}+00$ & $0.00 \mathrm{E}+00$ & $0.00 \mathrm{E}+00$ \\
\hline CA 45 & $1.05 \mathrm{E}-09$ & $1.05 \mathrm{E}-09$ & $1.04 \mathrm{E}-09$ & $1.01 \mathrm{E}-09$ & $9.27 \mathrm{E}-10$ & $7.18 \mathrm{E}-10$ & $6.06 \mathrm{E}-10$ & $4.90 \mathrm{E}-10$ & $2.28 \mathrm{E}-10$ \\
\hline CA 47 & $6.95 \mathrm{E}-09$ & $5.96 \mathrm{E}-09$ & $5.12 \mathrm{E}-09$ & $1.51 \mathrm{E}-09$ & 7.09E-11 & $7.39 \mathrm{E}-15$ & $1.54 \mathrm{E}-17$ & $7.37 \mathrm{E}-21$ & $0.00 \mathrm{E}+00$ \\
\hline $\mathrm{SC} 46$ & $3.46 \mathrm{E}-12$ & $3.44 \mathrm{E}-12$ & $3.41 \mathrm{E}-12$ & $3.19 \mathrm{E}-12$ & $2.70 \mathrm{E}-12$ & $1.65 \mathrm{E}-12$ & $1.18 \mathrm{E}-12$ & $7.82 \mathrm{E}-13$ & $1.76 \mathrm{E}-13$ \\
\hline $\mathrm{SC} 46 \mathrm{M}$ & $4.37 \mathrm{E}-12$ & $0.00 \mathrm{E}+00$ & $0.00 \mathrm{E}+00$ & $0.00 \mathrm{E}+00$ & $0.00 \mathrm{E}+00$ & $0.00 \mathrm{E}+00$ & $0.00 \mathrm{E}+00$ & $0.00 \mathrm{E}+00$ & $0.00 \mathrm{E}+00$ \\
\hline $\mathrm{SC} 47$ & $2.97 \mathrm{E}-05$ & $2.42 \mathrm{E}-05$ & $1.97 \mathrm{E}-05$ & $3.76 \mathrm{E}-06$ & $6.02 \mathrm{E}-08$ & $2.72 \mathrm{E}-13$ & $1.32 \mathrm{E}-16$ & $3.06 \mathrm{E}-20$ & $0.00 \mathrm{E}+00$ \\
\hline $\mathrm{SC} 48$ & $6.75 \mathrm{E}-05$ & 4.62E-05 & $3.16 \mathrm{E}-05$ & $1.51 \mathrm{E}-06$ & $7.61 \mathrm{E}-10$ & $9.68 \mathrm{E}-20$ & $2.45 \mathrm{E}-26$ & $0.00 \mathrm{E}+00$ & $0.00 \mathrm{E}+00$ \\
\hline SC 49 & $8.13 \mathrm{E}-06$ & $2.35 \mathrm{E}-13$ & $6.80 \mathrm{E}-21$ & $0.00 \mathrm{E}+00$ & $0.00 \mathrm{E}+00$ & $0.00 \mathrm{E}+00$ & $0.00 \mathrm{E}+00$ & $0.00 \mathrm{E}+00$ & $0.00 \mathrm{E}+00$ \\
\hline SC 50 & $6.95 \mathrm{E}-07$ & $0.00 \mathrm{E}+00$ & $0.00 \mathrm{E}+00$ & $0.00 \mathrm{E}+00$ & $0.00 \mathrm{E}+00$ & $0.00 \mathrm{E}+00$ & $0.00 \mathrm{E}+00$ & $0.00 \mathrm{E}+00$ & $0.00 \mathrm{E}+00$ \\
\hline TI 51 & $1.57 \mathrm{E}-02$ & $0.00 \mathrm{E}+00$ & $0.00 \mathrm{E}+00$ & $0.00 \mathrm{E}+00$ & $0.00 \mathrm{E}+00$ & $0.00 \mathrm{E}+00$ & $0.00 \mathrm{E}+00$ & $0.00 \mathrm{E}+00$ & $0.00 \mathrm{E}+00$ \\
\hline V 50 & $1.44 \mathrm{E}-16$ & $1.44 \mathrm{E}-16$ & $1.44 \mathrm{E}-16$ & $1.44 \mathrm{E}-16$ & $1.44 \mathrm{E}-16$ & $1.44 \mathrm{E}-16$ & $1.44 \mathrm{E}-16$ & $1.44 \mathrm{E}-16$ & $1.44 \mathrm{E}-16$ \\
\hline V 52 & $4.27 \mathrm{E}+01$ & $0.00 \mathrm{E}+00$ & $0.00 \mathrm{E}+00$ & $0.00 \mathrm{E}+00$ & $0.00 \mathrm{E}+00$ & $0.00 \mathrm{E}+00$ & $0.00 \mathrm{E}+00$ & $0.00 \mathrm{E}+00$ & $0.00 \mathrm{E}+00$ \\
\hline V 53 & $1.04 \mathrm{E}-01$ & $0.00 \mathrm{E}+00$ & $0.00 \mathrm{E}+00$ & $0.00 \mathrm{E}+00$ & $0.00 \mathrm{E}+00$ & $0.00 \mathrm{E}+00$ & $0.00 \mathrm{E}+00$ & $0.00 \mathrm{E}+00$ & $0.00 \mathrm{E}+00$ \\
\hline V 54 & $1.13 \mathrm{E}-03$ & $0.00 \mathrm{E}+00$ & $0.00 \mathrm{E}+00$ & $0.00 \mathrm{E}+00$ & $0.00 \mathrm{E}+00$ & $0.00 \mathrm{E}+00$ & $0.00 \mathrm{E}+00$ & $0.00 \mathrm{E}+00$ & $0.00 \mathrm{E}+00$ \\
\hline CR 51 & $1.92 \mathrm{E}+03$ & $1.87 \mathrm{E}+03$ & $1.82 \mathrm{E}+03$ & $1.49 \mathrm{E}+03$ & $9.04 \mathrm{E}+02$ & $2.02 \mathrm{E}+02$ & $7.41 \mathrm{E}+01$ & $2.12 \mathrm{E}+01$ & $2.35 \mathrm{E}-01$ \\
\hline
\end{tabular}


BSU-8242 3 DPA As-Run Physics Analysis

\begin{tabular}{|c|c|c|c|c|c|c|c|c|c|}
\hline \multicolumn{10}{|c|}{ ACTIVATION PRODUCTS (Ci) } \\
\hline ISOTOPE & EOC & 1 Day & 2 Days & 10 Days & 30 Days & 90 Days & 130 Days & 180 Days & 360 Days \\
\hline CR 55 & $3.14 \mathrm{E}+01$ & $0.00 \mathrm{E}+00$ & $0.00 \mathrm{E}+00$ & $0.00 \mathrm{E}+00$ & $0.00 \mathrm{E}+00$ & $0.00 \mathrm{E}+00$ & $0.00 \mathrm{E}+00$ & $0.00 \mathrm{E}+00$ & $0.00 \mathrm{E}+00$ \\
\hline MN 54 & $4.60 \mathrm{E}+01$ & $4.59 \mathrm{E}+01$ & $4.58 \mathrm{E}+01$ & $4.50 \mathrm{E}+01$ & $4.31 \mathrm{E}+01$ & $3.77 \mathrm{E}+01$ & $3.45 \mathrm{E}+01$ & $3.09 \mathrm{E}+01$ & $2.07 \mathrm{E}+01$ \\
\hline MN 56 & $2.51 \mathrm{E}+03$ & $3.97 \mathrm{E}+00$ & $6.24 \mathrm{E}-03$ & $0.00 \mathrm{E}+00$ & $0.00 \mathrm{E}+00$ & $0.00 \mathrm{E}+00$ & $0.00 \mathrm{E}+00$ & $0.00 \mathrm{E}+00$ & $0.00 \mathrm{E}+00$ \\
\hline MN 57 & $4.24 \mathrm{E}-01$ & $0.00 \mathrm{E}+00$ & $0.00 \mathrm{E}+00$ & $0.00 \mathrm{E}+00$ & $0.00 \mathrm{E}+00$ & $0.00 \mathrm{E}+00$ & $0.00 \mathrm{E}+00$ & $0.00 \mathrm{E}+00$ & $0.00 \mathrm{E}+00$ \\
\hline MN 58 & $1.26 \mathrm{E}-03$ & $0.00 \mathrm{E}+00$ & $0.00 \mathrm{E}+00$ & $0.00 \mathrm{E}+00$ & $0.00 \mathrm{E}+00$ & $0.00 \mathrm{E}+00$ & $0.00 \mathrm{E}+00$ & $0.00 \mathrm{E}+00$ & $0.00 \mathrm{E}+00$ \\
\hline FE 55 & $1.99 \mathrm{E}+02$ & $1.99 \mathrm{E}+02$ & $1.99 \mathrm{E}+02$ & $1.98 \mathrm{E}+02$ & $1.95 \mathrm{E}+02$ & $1.87 \mathrm{E}+02$ & $1.81 \mathrm{E}+02$ & $1.75 \mathrm{E}+02$ & $1.53 \mathrm{E}+02$ \\
\hline FE 59 & $3.78 \mathrm{E}+01$ & $3.72 \mathrm{E}+01$ & $3.66 \mathrm{E}+01$ & $3.24 \mathrm{E}+01$ & $2.38 \mathrm{E}+01$ & $9.44 \mathrm{E}+00$ & $5.10 \mathrm{E}+00$ & $2.36 \mathrm{E}+00$ & $1.48 \mathrm{E}-01$ \\
\hline $\mathrm{CO} 58$ & $7.03 \mathrm{E}+01$ & $6.97 \mathrm{E}+01$ & $6.90 \mathrm{E}+01$ & $6.38 \mathrm{E}+01$ & $5.24 \mathrm{E}+01$ & $2.91 \mathrm{E}+01$ & $1.97 \mathrm{E}+01$ & $1.21 \mathrm{E}+01$ & $2.07 \mathrm{E}+00$ \\
\hline $\mathrm{CO} 60$ & $5.09 \mathrm{E}-01$ & $5.08 \mathrm{E}-01$ & $5.08 \mathrm{E}-01$ & $5.07 \mathrm{E}-01$ & $5.03 \mathrm{E}-01$ & $4.92 \mathrm{E}-01$ & $4.85 \mathrm{E}-01$ & $4.77 \mathrm{E}-01$ & $4.47 \mathrm{E}-01$ \\
\hline $\mathrm{CO} 60 \mathrm{M}$ & $6.30 \mathrm{E}+00$ & $0.00 \mathrm{E}+00$ & $0.00 \mathrm{E}+00$ & $0.00 \mathrm{E}+00$ & $0.00 \mathrm{E}+00$ & $0.00 \mathrm{E}+00$ & $0.00 \mathrm{E}+00$ & $0.00 \mathrm{E}+00$ & $0.00 \mathrm{E}+00$ \\
\hline $\mathrm{CO} 61$ & $1.50 \mathrm{E}-01$ & $6.29 \mathrm{E}-06$ & $2.63 \mathrm{E}-10$ & $0.00 \mathrm{E}+00$ & $0.00 \mathrm{E}+00$ & $0.00 \mathrm{E}+00$ & $0.00 \mathrm{E}+00$ & $0.00 \mathrm{E}+00$ & $0.00 \mathrm{E}+00$ \\
\hline $\mathrm{CO} 62$ & $2.19 \mathrm{E}-02$ & $0.00 \mathrm{E}+00$ & $0.00 \mathrm{E}+00$ & $0.00 \mathrm{E}+00$ & $0.00 \mathrm{E}+00$ & $0.00 \mathrm{E}+00$ & $0.00 \mathrm{E}+00$ & $0.00 \mathrm{E}+00$ & $0.00 \mathrm{E}+00$ \\
\hline NI 59 & $2.44 \mathrm{E}-02$ & $2.44 \mathrm{E}-02$ & $2.44 \mathrm{E}-02$ & $2.44 \mathrm{E}-02$ & $2.44 \mathrm{E}-02$ & $2.44 \mathrm{E}-02$ & $2.44 \mathrm{E}-02$ & $2.44 \mathrm{E}-02$ & $2.44 \mathrm{E}-02$ \\
\hline NI 63 & $3.21 \mathrm{E}+00$ & $3.21 \mathrm{E}+00$ & $3.21 \mathrm{E}+00$ & $3.21 \mathrm{E}+00$ & $3.21 \mathrm{E}+00$ & $3.20 \mathrm{E}+00$ & $3.20 \mathrm{E}+00$ & $3.20 \mathrm{E}+00$ & $3.18 \mathrm{E}+00$ \\
\hline NI 65 & $2.52 \mathrm{E}+01$ & $3.43 \mathrm{E}-02$ & 4.65E-05 & $0.00 \mathrm{E}+00$ & $0.00 \mathrm{E}+00$ & $0.00 \mathrm{E}+00$ & $0.00 \mathrm{E}+00$ & $0.00 \mathrm{E}+00$ & $0.00 \mathrm{E}+00$ \\
\hline NI 66 & $6.88 \mathrm{E}-04$ & $5.08 \mathrm{E}-04$ & $3.74 \mathrm{E}-04$ & $3.27 \mathrm{E}-05$ & $7.40 \mathrm{E}-08$ & $8.54 \mathrm{E}-16$ & $4.36 \mathrm{E}-21$ & $1.06 \mathrm{E}-27$ & $0.00 \mathrm{E}+00$ \\
\hline CU 64 & $3.88 \mathrm{E}+01$ & $1.05 \mathrm{E}+01$ & $2.83 \mathrm{E}+00$ & $7.95 \mathrm{E}-05$ & $3.33 \mathrm{E}-16$ & $0.00 \mathrm{E}+00$ & $0.00 \mathrm{E}+00$ & $0.00 \mathrm{E}+00$ & $0.00 \mathrm{E}+00$ \\
\hline CU 66 & $8.48 \mathrm{E}+00$ & $5.08 \mathrm{E}-04$ & $3.75 \mathrm{E}-04$ & $3.28 \mathrm{E}-05$ & $7.41 \mathrm{E}-08$ & $8.55 \mathrm{E}-16$ & $4.37 \mathrm{E}-21$ & $1.06 \mathrm{E}-27$ & $0.00 \mathrm{E}+00$ \\
\hline CU 67 & 4.33E-05 & $3.31 \mathrm{E}-05$ & $2.53 \mathrm{E}-05$ & $2.94 \mathrm{E}-06$ & $1.36 \mathrm{E}-08$ & $1.34 \mathrm{E}-15$ & $2.84 \mathrm{E}-20$ & $4.12 \mathrm{E}-26$ & $0.00 \mathrm{E}+00$ \\
\hline ZN 65 & $4.16 \mathrm{E}-03$ & 4.15E-03 & 4.13E-03 & 4.04E-03 & $3.82 \mathrm{E}-03$ & $3.22 \mathrm{E}-03$ & $2.87 \mathrm{E}-03$ & $2.49 \mathrm{E}-03$ & $1.49 \mathrm{E}-03$ \\
\hline ZN 69 & $6.75 \mathrm{E}-08$ & $1.45 \mathrm{E}-09$ & $4.34 \mathrm{E}-10$ & $2.74 \mathrm{E}-14$ & $8.66 \mathrm{E}-25$ & $0.00 \mathrm{E}+00$ & $0.00 \mathrm{E}+00$ & $0.00 \mathrm{E}+00$ & $0.00 \mathrm{E}+00$ \\
\hline ZN 69M & 4.54E-09 & $1.35 \mathrm{E}-09$ & $4.04 \mathrm{E}-10$ & $2.55 \mathrm{E}-14$ & $8.06 \mathrm{E}-25$ & $0.00 \mathrm{E}+00$ & $0.00 \mathrm{E}+00$ & $0.00 \mathrm{E}+00$ & $0.00 \mathrm{E}+00$ \\
\hline GA 70 & $1.28 \mathrm{E}-10$ & $3.65 \mathrm{E}-31$ & $0.00 \mathrm{E}+00$ & $0.00 \mathrm{E}+00$ & $0.00 \mathrm{E}+00$ & $0.00 \mathrm{E}+00$ & $0.00 \mathrm{E}+00$ & $0.00 \mathrm{E}+00$ & $0.00 \mathrm{E}+00$ \\
\hline GA 72 & $2.20 \mathrm{E}-16$ & $5.34 \mathrm{E}-17$ & $2.76 \mathrm{E}-17$ & $2.19 \mathrm{E}-21$ & $1.14 \mathrm{E}-31$ & $0.00 \mathrm{E}+00$ & $0.00 \mathrm{E}+00$ & $0.00 \mathrm{E}+00$ & $0.00 \mathrm{E}+00$ \\
\hline GE 71 & $8.30 \mathrm{E}-14$ & $7.83 \mathrm{E}-14$ & $7.38 \mathrm{E}-14$ & $4.61 \mathrm{E}-14$ & $1.43 \mathrm{E}-14$ & $4.20 \mathrm{E}-16$ & $3.95 \mathrm{E}-17$ & $1.69 \mathrm{E}-18$ & $4.32 \mathrm{E}-23$ \\
\hline GE $71 \mathrm{M}$ & $1.02 \mathrm{E}-14$ & $0.00 \mathrm{E}+00$ & $0.00 \mathrm{E}+00$ & $0.00 \mathrm{E}+00$ & $0.00 \mathrm{E}+00$ & $0.00 \mathrm{E}+00$ & $0.00 \mathrm{E}+00$ & $0.00 \mathrm{E}+00$ & $0.00 \mathrm{E}+00$ \\
\hline
\end{tabular}


BSU-8242 3 DPA As-Run Physics Analysis

\begin{tabular}{|c|c|c|c|c|c|c|c|c|c|}
\hline \multicolumn{10}{|c|}{ ACTIVATION PRODUCTS (Ci) } \\
\hline ISOTOPE & EOC & 1 Day & 2 Days & 10 Days & 30 Days & 90 Days & 130 Days & 180 Days & 360 Days \\
\hline SR 89 & 4.05E-08 & $3.99 \mathrm{E}-08$ & $3.94 \mathrm{E}-08$ & $3.53 \mathrm{E}-08$ & $2.68 \mathrm{E}-08$ & $1.18 \mathrm{E}-08$ & $6.80 \mathrm{E}-09$ & $3.42 \mathrm{E}-09$ & $2.89 \mathrm{E}-10$ \\
\hline SR 90 & $1.15 \mathrm{E}-11$ & $1.15 \mathrm{E}-11$ & $1.15 \mathrm{E}-11$ & $1.15 \mathrm{E}-11$ & $1.15 \mathrm{E}-11$ & $1.15 \mathrm{E}-11$ & $1.14 \mathrm{E}-11$ & $1.14 \mathrm{E}-11$ & $1.13 \mathrm{E}-11$ \\
\hline SR 91 & $1.21 \mathrm{E}-09$ & $2.10 \mathrm{E}-10$ & $3.64 \mathrm{E}-11$ & $3.00 \mathrm{E}-17$ & $2.28 \mathrm{E}-32$ & $0.00 \mathrm{E}+00$ & $0.00 \mathrm{E}+00$ & $0.00 \mathrm{E}+00$ & $0.00 \mathrm{E}+00$ \\
\hline SR 93 & $2.08 \mathrm{E}-15$ & $0.00 \mathrm{E}+00$ & $0.00 \mathrm{E}+00$ & $0.00 \mathrm{E}+00$ & $0.00 \mathrm{E}+00$ & $0.00 \mathrm{E}+00$ & $0.00 \mathrm{E}+00$ & $0.00 \mathrm{E}+00$ & $0.00 \mathrm{E}+00$ \\
\hline Y 89M & $7.10 \mathrm{E}-04$ & $5.74 \mathrm{E}-04$ & 4.64E-04 & $8.51 \mathrm{E}-05$ & $1.23 \mathrm{E}-06$ & $3.65 \mathrm{E}-12$ & $7.55 \mathrm{E}-16$ & $1.88 \mathrm{E}-20$ & $0.00 \mathrm{E}+00$ \\
\hline Y 90 & $1.06 \mathrm{E}-04$ & $8.16 \mathrm{E}-05$ & $6.29 \mathrm{E}-05$ & $7.86 \mathrm{E}-06$ & $4.34 \mathrm{E}-08$ & $1.15 \mathrm{E}-11$ & $1.14 \mathrm{E}-11$ & $1.14 \mathrm{E}-11$ & $1.13 \mathrm{E}-11$ \\
\hline Y 90M & $1.01 \mathrm{E}-09$ & $4.70 \mathrm{E}-12$ & $2.19 \mathrm{E}-14$ & $0.00 \mathrm{E}+00$ & $0.00 \mathrm{E}+00$ & $0.00 \mathrm{E}+00$ & $0.00 \mathrm{E}+00$ & $0.00 \mathrm{E}+00$ & $0.00 \mathrm{E}+00$ \\
\hline Y 91 & $1.56 \mathrm{E}-08$ & $1.54 \mathrm{E}-08$ & $1.52 \mathrm{E}-08$ & $1.39 \mathrm{E}-08$ & $1.09 \mathrm{E}-08$ & $5.37 \mathrm{E}-09$ & $3.34 \mathrm{E}-09$ & $1.85 \mathrm{E}-09$ & $2.19 \mathrm{E}-10$ \\
\hline Y 92 & $1.55 \mathrm{E}-07$ & $1.41 \mathrm{E}-09$ & $1.28 \mathrm{E}-11$ & 5.97E-28 & $0.00 \mathrm{E}+00$ & $0.00 \mathrm{E}+00$ & $0.00 \mathrm{E}+00$ & $0.00 \mathrm{E}+00$ & $0.00 \mathrm{E}+00$ \\
\hline Y 93 & $2.08 \mathrm{E}-15$ & $4.16 \mathrm{E}-16$ & $7.09 \mathrm{E}-17$ & $1.34 \mathrm{E}-22$ & $0.00 \mathrm{E}+00$ & $0.00 \mathrm{E}+00$ & $0.00 \mathrm{E}+00$ & $0.00 \mathrm{E}+00$ & $0.00 \mathrm{E}+00$ \\
\hline Y 94 & $8.63 \mathrm{E}-10$ & $0.00 \mathrm{E}+00$ & $0.00 \mathrm{E}+00$ & $0.00 \mathrm{E}+00$ & $0.00 \mathrm{E}+00$ & $0.00 \mathrm{E}+00$ & $0.00 \mathrm{E}+00$ & $0.00 \mathrm{E}+00$ & $0.00 \mathrm{E}+00$ \\
\hline Y 96 & $2.76 \mathrm{E}-15$ & $0.00 \mathrm{E}+00$ & $0.00 \mathrm{E}+00$ & $0.00 \mathrm{E}+00$ & $0.00 \mathrm{E}+00$ & $0.00 \mathrm{E}+00$ & $0.00 \mathrm{E}+00$ & $0.00 \mathrm{E}+00$ & $0.00 \mathrm{E}+00$ \\
\hline ZR 89 & $7.11 \mathrm{E}-04$ & $5.75 \mathrm{E}-04$ & 4.65E-04 & $8.53 \mathrm{E}-05$ & $1.23 \mathrm{E}-06$ & $3.65 \mathrm{E}-12$ & $7.56 \mathrm{E}-16$ & $1.88 \mathrm{E}-20$ & $0.00 \mathrm{E}+00$ \\
\hline ZR 93 & $1.99 \mathrm{E}-09$ & $1.99 \mathrm{E}-09$ & $1.99 \mathrm{E}-09$ & $1.99 \mathrm{E}-09$ & $1.99 \mathrm{E}-09$ & $1.99 \mathrm{E}-09$ & $1.99 \mathrm{E}-09$ & $1.99 \mathrm{E}-09$ & $1.99 \mathrm{E}-09$ \\
\hline ZR 95 & $1.64 \mathrm{E}-03$ & $1.63 \mathrm{E}-03$ & $1.61 \mathrm{E}-03$ & $1.48 \mathrm{E}-03$ & $1.19 \mathrm{E}-03$ & $6.20 \mathrm{E}-04$ & $4.02 \mathrm{E}-04$ & $2.34 \mathrm{E}-04$ & $3.33 \mathrm{E}-05$ \\
\hline ZR 97 & $5.62 \mathrm{E}-05$ & $2.10 \mathrm{E}-05$ & $7.84 \mathrm{E}-06$ & $2.98 \mathrm{E}-09$ & $8.40 \mathrm{E}-18$ & $0.00 \mathrm{E}+00$ & $0.00 \mathrm{E}+00$ & $0.00 \mathrm{E}+00$ & $0.00 \mathrm{E}+00$ \\
\hline NB 92 & $1.40 \mathrm{E}-01$ & $1.30 \mathrm{E}-01$ & $1.22 \mathrm{E}-01$ & $7.05 \mathrm{E}-02$ & $1.80 \mathrm{E}-02$ & $3.01 \mathrm{E}-04$ & $1.96 \mathrm{E}-05$ & $6.48 \mathrm{E}-07$ & $3.01 \mathrm{E}-12$ \\
\hline NB 93M & $4.46 \mathrm{E}-11$ & $4.48 \mathrm{E}-11$ & $4.51 \mathrm{E}-11$ & $4.71 \mathrm{E}-11$ & $5.23 \mathrm{E}-11$ & $6.76 \mathrm{E}-11$ & $7.78 \mathrm{E}-11$ & $9.04 \mathrm{E}-11$ & $1.35 \mathrm{E}-10$ \\
\hline NB 94 & $3.80 \mathrm{E}-05$ & $3.80 \mathrm{E}-05$ & $3.80 \mathrm{E}-05$ & $3.80 \mathrm{E}-05$ & $3.80 \mathrm{E}-05$ & $3.80 \mathrm{E}-05$ & $3.80 \mathrm{E}-05$ & $3.80 \mathrm{E}-05$ & $3.80 \mathrm{E}-05$ \\
\hline NB 95 & $9.69 \mathrm{E}-02$ & $9.50 \mathrm{E}-02$ & $9.32 \mathrm{E}-02$ & $7.98 \mathrm{E}-02$ & $5.42 \mathrm{E}-02$ & $1.72 \mathrm{E}-02$ & $8.07 \mathrm{E}-03$ & $3.20 \mathrm{E}-03$ & $1.51 \mathrm{E}-04$ \\
\hline NB 95M & $1.17 \mathrm{E}-05$ & $1.17 \mathrm{E}-05$ & $1.16 \mathrm{E}-05$ & $1.09 \mathrm{E}-05$ & $8.81 \mathrm{E}-06$ & $4.60 \mathrm{E}-06$ & $2.98 \mathrm{E}-06$ & $1.74 \mathrm{E}-06$ & $2.47 \mathrm{E}-07$ \\
\hline NB 96 & $5.46 \mathrm{E}-03$ & $2.68 \mathrm{E}-03$ & $1.31 \mathrm{E}-03$ & $4.40 \mathrm{E}-06$ & $2.85 \mathrm{E}-12$ & $7.76 \mathrm{E}-31$ & $0.00 \mathrm{E}+00$ & $0.00 \mathrm{E}+00$ & $0.00 \mathrm{E}+00$ \\
\hline NB 97 & $2.00 \mathrm{E}-03$ & $2.11 \mathrm{E}-05$ & $7.88 \mathrm{E}-06$ & $3.21 \mathrm{E}-09$ & $9.06 \mathrm{E}-18$ & $0.00 \mathrm{E}+00$ & $0.00 \mathrm{E}+00$ & $0.00 \mathrm{E}+00$ & $0.00 \mathrm{E}+00$ \\
\hline NB 97M & $5.32 \mathrm{E}-05$ & $1.99 \mathrm{E}-05$ & $7.43 \mathrm{E}-06$ & 2.82E-09 & $7.96 \mathrm{E}-18$ & $0.00 \mathrm{E}+00$ & $0.00 \mathrm{E}+00$ & $0.00 \mathrm{E}+00$ & $0.00 \mathrm{E}+00$ \\
\hline NB 98 & $2.97 \mathrm{E}-04$ & $0.00 \mathrm{E}+00$ & $0.00 \mathrm{E}+00$ & $0.00 \mathrm{E}+00$ & $0.00 \mathrm{E}+00$ & $0.00 \mathrm{E}+00$ & $0.00 \mathrm{E}+00$ & $0.00 \mathrm{E}+00$ & $0.00 \mathrm{E}+00$ \\
\hline NB100 & $1.48 \mathrm{E}-05$ & $0.00 \mathrm{E}+00$ & $0.00 \mathrm{E}+00$ & $0.00 \mathrm{E}+00$ & $0.00 \mathrm{E}+00$ & $0.00 \mathrm{E}+00$ & $0.00 \mathrm{E}+00$ & $0.00 \mathrm{E}+00$ & $0.00 \mathrm{E}+00$ \\
\hline
\end{tabular}


BSU-8242 3 DPA As-Run Physics Analysis

\begin{tabular}{|c|c|c|c|c|c|c|c|c|c|}
\hline \multicolumn{10}{|c|}{ ACTIVATION PRODUCTS (Ci) } \\
\hline ISOTOPE & EOC & 1 Day & 2 Days & 10 Days & 30 Days & 90 Days & 130 Days & 180 Days & 360 Days \\
\hline MO 93M & 4.11E-02 & $3.62 \mathrm{E}-03$ & $3.19 \mathrm{E}-04$ & $1.17 \mathrm{E}-12$ & $0.00 \mathrm{E}+00$ & $0.00 \mathrm{E}+00$ & $0.00 \mathrm{E}+00$ & $0.00 \mathrm{E}+00$ & $0.00 \mathrm{E}+00$ \\
\hline MO 93 & $1.24 \mathrm{E}-04$ & $1.24 \mathrm{E}-04$ & $1.24 \mathrm{E}-04$ & $1.24 \mathrm{E}-04$ & $1.24 \mathrm{E}-04$ & $1.24 \mathrm{E}-04$ & $1.24 \mathrm{E}-04$ & $1.24 \mathrm{E}-04$ & $1.24 \mathrm{E}-04$ \\
\hline MO 99 & $2.88 \mathrm{E}+01$ & $2.24 \mathrm{E}+01$ & $1.74 \mathrm{E}+01$ & $2.31 \mathrm{E}+00$ & $1.50 \mathrm{E}-02$ & 4.05E-09 & $1.69 \mathrm{E}-13$ & $5.69 \mathrm{E}-19$ & $0.00 \mathrm{E}+00$ \\
\hline MO101 & $7.15 \mathrm{E}+00$ & $0.00 \mathrm{E}+00$ & $0.00 \mathrm{E}+00$ & $0.00 \mathrm{E}+00$ & $0.00 \mathrm{E}+00$ & $0.00 \mathrm{E}+00$ & $0.00 \mathrm{E}+00$ & $0.00 \mathrm{E}+00$ & $0.00 \mathrm{E}+00$ \\
\hline TC 99 & $5.27 \mathrm{E}-06$ & $5.30 \mathrm{E}-06$ & $5.32 \mathrm{E}-06$ & $5.38 \mathrm{E}-06$ & $5.39 \mathrm{E}-06$ & $5.39 \mathrm{E}-06$ & $5.39 \mathrm{E}-06$ & $5.39 \mathrm{E}-06$ & $5.39 \mathrm{E}-06$ \\
\hline $\mathrm{TC} 100$ & 4.35E-01 & $0.00 \mathrm{E}+00$ & $0.00 \mathrm{E}+00$ & $0.00 \mathrm{E}+00$ & $0.00 \mathrm{E}+00$ & $0.00 \mathrm{E}+00$ & $0.00 \mathrm{E}+00$ & $0.00 \mathrm{E}+00$ & $0.00 \mathrm{E}+00$ \\
\hline $\mathrm{TC} 101$ & $7.15 \mathrm{E}+00$ & $0.00 \mathrm{E}+00$ & $0.00 \mathrm{E}+00$ & $0.00 \mathrm{E}+00$ & $0.00 \mathrm{E}+00$ & $0.00 \mathrm{E}+00$ & $0.00 \mathrm{E}+00$ & $0.00 \mathrm{E}+00$ & $0.00 \mathrm{E}+00$ \\
\hline RU103 & $3.03 \mathrm{E}-04$ & $2.98 \mathrm{E}-04$ & $2.93 \mathrm{E}-04$ & $2.54 \mathrm{E}-04$ & $1.79 \mathrm{E}-04$ & $6.19 \mathrm{E}-05$ & $3.06 \mathrm{E}-05$ & $1.27 \mathrm{E}-05$ & $5.28 \mathrm{E}-07$ \\
\hline RU105 & $3.54 \mathrm{E}-09$ & $8.34 \mathrm{E}-11$ & $1.97 \mathrm{E}-12$ & $1.88 \mathrm{E}-25$ & $0.00 \mathrm{E}+00$ & $0.00 \mathrm{E}+00$ & $0.00 \mathrm{E}+00$ & $0.00 \mathrm{E}+00$ & $0.00 \mathrm{E}+00$ \\
\hline RU106 & $1.44 \mathrm{E}-15$ & $1.43 \mathrm{E}-15$ & $1.43 \mathrm{E}-15$ & $1.41 \mathrm{E}-15$ & $1.36 \mathrm{E}-15$ & $1.21 \mathrm{E}-15$ & $1.12 \mathrm{E}-15$ & $1.02 \mathrm{E}-15$ & $7.29 \mathrm{E}-16$ \\
\hline RH104 & 4.79E-06 & $0.00 \mathrm{E}+00$ & $0.00 \mathrm{E}+00$ & $0.00 \mathrm{E}+00$ & $0.00 \mathrm{E}+00$ & $0.00 \mathrm{E}+00$ & $0.00 \mathrm{E}+00$ & $0.00 \mathrm{E}+00$ & $0.00 \mathrm{E}+00$ \\
\hline RH104M & $3.47 \mathrm{E}-07$ & $0.00 \mathrm{E}+00$ & $0.00 \mathrm{E}+00$ & $0.00 \mathrm{E}+00$ & $0.00 \mathrm{E}+00$ & $0.00 \mathrm{E}+00$ & $0.00 \mathrm{E}+00$ & $0.00 \mathrm{E}+00$ & $0.00 \mathrm{E}+00$ \\
\hline RH105 & 2.82E-09 & $2.07 \mathrm{E}-09$ & $1.30 \mathrm{E}-09$ & $3.01 \mathrm{E}-11$ & $2.47 \mathrm{E}-15$ & $1.36 \mathrm{E}-27$ & $0.00 \mathrm{E}+00$ & $0.00 \mathrm{E}+00$ & $0.00 \mathrm{E}+00$ \\
\hline RH105M & $9.91 \mathrm{E}-10$ & $2.34 \mathrm{E}-11$ & $5.52 \mathrm{E}-13$ & $5.26 \mathrm{E}-26$ & $0.00 \mathrm{E}+00$ & $0.00 \mathrm{E}+00$ & $0.00 \mathrm{E}+00$ & $0.00 \mathrm{E}+00$ & $0.00 \mathrm{E}+00$ \\
\hline RH106 & 4.91E-10 & $1.43 \mathrm{E}-15$ & $1.43 \mathrm{E}-15$ & $1.41 \mathrm{E}-15$ & $1.36 \mathrm{E}-15$ & $1.21 \mathrm{E}-15$ & $1.12 \mathrm{E}-15$ & $1.02 \mathrm{E}-15$ & $7.29 \mathrm{E}-16$ \\
\hline RH106M & $2.39 \mathrm{E}-10$ & $1.24 \mathrm{E}-13$ & $6.45 \mathrm{E}-17$ & $0.00 \mathrm{E}+00$ & $0.00 \mathrm{E}+00$ & $0.00 \mathrm{E}+00$ & $0.00 \mathrm{E}+00$ & $0.00 \mathrm{E}+00$ & $0.00 \mathrm{E}+00$ \\
\hline PD107M & $1.75 \mathrm{E}-14$ & $0.00 \mathrm{E}+00$ & $0.00 \mathrm{E}+00$ & $0.00 \mathrm{E}+00$ & $0.00 \mathrm{E}+00$ & $0.00 \mathrm{E}+00$ & $0.00 \mathrm{E}+00$ & $0.00 \mathrm{E}+00$ & $0.00 \mathrm{E}+00$ \\
\hline TM172 & $1.66 \mathrm{E}-09$ & $1.28 \mathrm{E}-09$ & $9.84 \mathrm{E}-10$ & $1.21 \mathrm{E}-10$ & $6.49 \mathrm{E}-13$ & $9.92 \mathrm{E}-20$ & $2.84 \mathrm{E}-24$ & $5.93 \mathrm{E}-30$ & $0.00 \mathrm{E}+00$ \\
\hline TM173 & $1.42 \mathrm{E}-09$ & $1.89 \mathrm{E}-10$ & $2.51 \mathrm{E}-11$ & $2.43 \mathrm{E}-18$ & $0.00 \mathrm{E}+00$ & $0.00 \mathrm{E}+00$ & $0.00 \mathrm{E}+00$ & $0.00 \mathrm{E}+00$ & $0.00 \mathrm{E}+00$ \\
\hline YB175 & $1.96 \mathrm{E}-12$ & $1.66 \mathrm{E}-12$ & $1.41 \mathrm{E}-12$ & $3.74 \mathrm{E}-13$ & $1.37 \mathrm{E}-14$ & $6.69 \mathrm{E}-19$ & $0.00 \mathrm{E}+00$ & $0.00 \mathrm{E}+00$ & $0.00 \mathrm{E}+00$ \\
\hline YB175M & $1.38 \mathrm{E}-12$ & $0.00 \mathrm{E}+00$ & $0.00 \mathrm{E}+00$ & $0.00 \mathrm{E}+00$ & $0.00 \mathrm{E}+00$ & $0.00 \mathrm{E}+00$ & $0.00 \mathrm{E}+00$ & $0.00 \mathrm{E}+00$ & $0.00 \mathrm{E}+00$ \\
\hline LU176 & $6.07 \mathrm{E}-15$ & $6.07 \mathrm{E}-15$ & $6.07 \mathrm{E}-15$ & $6.07 \mathrm{E}-15$ & $6.07 \mathrm{E}-15$ & $6.07 \mathrm{E}-15$ & $6.07 \mathrm{E}-15$ & $6.07 \mathrm{E}-15$ & $6.07 \mathrm{E}-15$ \\
\hline LU176M & 4.92E-03 & $5.42 \mathrm{E}-05$ & $5.97 \mathrm{E}-07$ & $1.30 \mathrm{E}-22$ & $0.00 \mathrm{E}+00$ & $0.00 \mathrm{E}+00$ & $0.00 \mathrm{E}+00$ & $0.00 \mathrm{E}+00$ & $0.00 \mathrm{E}+00$ \\
\hline LU177 & $1.44 \mathrm{E}-03$ & $1.30 \mathrm{E}-03$ & $1.17 \mathrm{E}-03$ & $5.12 \mathrm{E}-04$ & $6.50 \mathrm{E}-05$ & $3.26 \mathrm{E}-07$ & $1.65 \mathrm{E}-07$ & $1.30 \mathrm{E}-07$ & $5.82 \mathrm{E}-08$ \\
\hline LU177M & $1.27 \mathrm{E}-06$ & $1.26 \mathrm{E}-06$ & $1.26 \mathrm{E}-06$ & $1.21 \mathrm{E}-06$ & $1.11 \mathrm{E}-06$ & $8.47 \mathrm{E}-07$ & $7.08 \mathrm{E}-07$ & $5.66 \mathrm{E}-07$ & $2.53 \mathrm{E}-07$ \\
\hline HF 175 & $1.93 \mathrm{E}-02$ & $1.91 \mathrm{E}-02$ & $1.89 \mathrm{E}-02$ & $1.75 \mathrm{E}-02$ & $1.43 \mathrm{E}-02$ & $7.91 \mathrm{E}-03$ & $5.32 \mathrm{E}-03$ & $3.24 \mathrm{E}-03$ & $5.46 \mathrm{E}-04$ \\
\hline
\end{tabular}


BSU-8242 3 DPA As-Run Physics Analysis

\begin{tabular}{|c|c|c|c|c|c|c|c|c|c|}
\hline \multicolumn{10}{|c|}{ ACTIVATION PRODUCTS (Ci) } \\
\hline ISOTOPE & EOC & 1 Day & 2 Days & 10 Days & 30 Days & 90 Days & 130 Days & 180 Days & 360 Days \\
\hline HF178M & $5.53 \mathrm{E}-03$ & $0.00 \mathrm{E}+00$ & $0.00 \mathrm{E}+00$ & $0.00 \mathrm{E}+00$ & $0.00 \mathrm{E}+00$ & $0.00 \mathrm{E}+00$ & $0.00 \mathrm{E}+00$ & $0.00 \mathrm{E}+00$ & $0.00 \mathrm{E}+00$ \\
\hline HF179M & $5.33 \mathrm{E}+00$ & $0.00 \mathrm{E}+00$ & $0.00 \mathrm{E}+00$ & $0.00 \mathrm{E}+00$ & $0.00 \mathrm{E}+00$ & $0.00 \mathrm{E}+00$ & $0.00 \mathrm{E}+00$ & $0.00 \mathrm{E}+00$ & $0.00 \mathrm{E}+00$ \\
\hline HF180M & 2.89E-02 & $1.41 \mathrm{E}-03$ & $6.83 \mathrm{E}-05$ & $2.12 \mathrm{E}-15$ & $0.00 \mathrm{E}+00$ & $0.00 \mathrm{E}+00$ & $0.00 \mathrm{E}+00$ & $0.00 \mathrm{E}+00$ & $0.00 \mathrm{E}+00$ \\
\hline HF181 & 4.19E-01 & 4.12E-01 & $4.06 \mathrm{E}-01$ & $3.56 \mathrm{E}-01$ & $2.57 \mathrm{E}-01$ & $9.62 \mathrm{E}-02$ & $5.00 \mathrm{E}-02$ & $2.21 \mathrm{E}-02$ & $1.16 \mathrm{E}-03$ \\
\hline HF182 & $1.60 \mathrm{E}-10$ & $1.60 \mathrm{E}-10$ & $1.60 \mathrm{E}-10$ & $1.60 \mathrm{E}-10$ & $1.60 \mathrm{E}-10$ & $1.60 \mathrm{E}-10$ & $1.60 \mathrm{E}-10$ & $1.60 \mathrm{E}-10$ & $1.60 \mathrm{E}-10$ \\
\hline TA180 & $2.35 \mathrm{E}-14$ & $2.35 \mathrm{E}-14$ & $2.35 \mathrm{E}-14$ & $2.35 \mathrm{E}-14$ & $2.35 \mathrm{E}-14$ & $2.35 \mathrm{E}-14$ & $2.35 \mathrm{E}-14$ & $2.35 \mathrm{E}-14$ & $2.35 \mathrm{E}-14$ \\
\hline TA182 & $5.46 \mathrm{E}+02$ & $5.43 \mathrm{E}+02$ & $5.39 \mathrm{E}+02$ & $5.14 \mathrm{E}+02$ & $4.56 \mathrm{E}+02$ & $3.17 \mathrm{E}+02$ & $2.49 \mathrm{E}+02$ & $1.84 \mathrm{E}+02$ & $6.23 \mathrm{E}+01$ \\
\hline TA182M & $2.67 \mathrm{E}+00$ & $0.00 \mathrm{E}+00$ & $0.00 \mathrm{E}+00$ & $0.00 \mathrm{E}+00$ & $0.00 \mathrm{E}+00$ & $0.00 \mathrm{E}+00$ & $0.00 \mathrm{E}+00$ & $0.00 \mathrm{E}+00$ & $0.00 \mathrm{E}+00$ \\
\hline TA183 & $5.10 \mathrm{E}+03$ & $4.45 \mathrm{E}+03$ & $3.89 \mathrm{E}+03$ & $1.31 \mathrm{E}+03$ & $8.65 \mathrm{E}+01$ & $2.49 \mathrm{E}-02$ & $1.08 \mathrm{E}-04$ & $1.20 \mathrm{E}-07$ & $2.86 \mathrm{E}-18$ \\
\hline W181 & $3.09 \mathrm{E}-15$ & $3.08 \mathrm{E}-15$ & $3.06 \mathrm{E}-15$ & $2.92 \mathrm{E}-15$ & $2.61 \mathrm{E}-15$ & $1.85 \mathrm{E}-15$ & $1.47 \mathrm{E}-15$ & $1.11 \mathrm{E}-15$ & $3.95 \mathrm{E}-16$ \\
\hline W183M & $6.04 \mathrm{E}-01$ & $0.00 \mathrm{E}+00$ & $0.00 \mathrm{E}+00$ & $0.00 \mathrm{E}+00$ & $0.00 \mathrm{E}+00$ & $0.00 \mathrm{E}+00$ & $0.00 \mathrm{E}+00$ & $0.00 \mathrm{E}+00$ & $0.00 \mathrm{E}+00$ \\
\hline W185 & 7.97E-01 & $7.90 \mathrm{E}-01$ & 7.83E-01 & 7.27E-01 & $6.05 \mathrm{E}-01$ & $3.48 \mathrm{E}-01$ & $2.40 \mathrm{E}-01$ & $1.51 \mathrm{E}-01$ & $2.88 \mathrm{E}-02$ \\
\hline W185M & $2.50 \mathrm{E}-03$ & $0.00 \mathrm{E}+00$ & $0.00 \mathrm{E}+00$ & $0.00 \mathrm{E}+00$ & $0.00 \mathrm{E}+00$ & $0.00 \mathrm{E}+00$ & $0.00 \mathrm{E}+00$ & $0.00 \mathrm{E}+00$ & $0.00 \mathrm{E}+00$ \\
\hline W187 & 4.87E-04 & $2.43 \mathrm{E}-04$ & $1.21 \mathrm{E}-04$ & 4.62E-07 & $4.16 \mathrm{E}-13$ & $2.97 \mathrm{E}-31$ & $0.00 \mathrm{E}+00$ & $0.00 \mathrm{E}+00$ & $0.00 \mathrm{E}+00$ \\
\hline W188 & $1.09 \mathrm{E}-06$ & $1.08 \mathrm{E}-06$ & $1.06 \mathrm{E}-06$ & $9.82 \mathrm{E}-07$ & $8.04 \mathrm{E}-07$ & $4.42 \mathrm{E}-07$ & $2.96 \mathrm{E}-07$ & $1.80 \mathrm{E}-07$ & $2.98 \mathrm{E}-08$ \\
\hline RE186 & $1.52 \mathrm{E}-01$ & $1.26 \mathrm{E}-01$ & $1.05 \mathrm{E}-01$ & $2.42 \mathrm{E}-02$ & $6.17 \mathrm{E}-04$ & $1.02 \mathrm{E}-08$ & $6.60 \mathrm{E}-12$ & $6.82 \mathrm{E}-16$ & $3.06 \mathrm{E}-30$ \\
\hline RE187 & $6.30 \mathrm{E}-16$ & $6.43 \mathrm{E}-16$ & $6.49 \mathrm{E}-16$ & $6.56 \mathrm{E}-16$ & $6.56 \mathrm{E}-16$ & $6.56 \mathrm{E}-16$ & $6.56 \mathrm{E}-16$ & $6.56 \mathrm{E}-16$ & $6.56 \mathrm{E}-16$ \\
\hline RE188 & $1.89 \mathrm{E}-05$ & 7.91E-06 & $3.64 \mathrm{E}-06$ & $9.94 \mathrm{E}-07$ & $8.13 \mathrm{E}-07$ & $4.46 \mathrm{E}-07$ & $2.99 \mathrm{E}-07$ & $1.82 \mathrm{E}-07$ & $3.01 \mathrm{E}-08$ \\
\hline RE188M & $1.75 \mathrm{E}-05$ & $0.00 \mathrm{E}+00$ & $0.00 \mathrm{E}+00$ & $0.00 \mathrm{E}+00$ & $0.00 \mathrm{E}+00$ & $0.00 \mathrm{E}+00$ & $0.00 \mathrm{E}+00$ & $0.00 \mathrm{E}+00$ & $0.00 \mathrm{E}+00$ \\
\hline RE189 & $2.86 \mathrm{E}-10$ & $1.44 \mathrm{E}-10$ & $7.28 \mathrm{E}-11$ & $3.05 \mathrm{E}-13$ & $3.45 \mathrm{E}-19$ & $0.00 \mathrm{E}+00$ & $0.00 \mathrm{E}+00$ & $0.00 \mathrm{E}+00$ & $0.00 \mathrm{E}+00$ \\
\hline OS190M & $6.99 \mathrm{E}-14$ & $0.00 \mathrm{E}+00$ & $0.00 \mathrm{E}+00$ & $0.00 \mathrm{E}+00$ & $0.00 \mathrm{E}+00$ & $0.00 \mathrm{E}+00$ & $0.00 \mathrm{E}+00$ & $0.00 \mathrm{E}+00$ & $0.00 \mathrm{E}+00$ \\
\hline OS191 & $9.25 \mathrm{E}-12$ & $9.19 \mathrm{E}-12$ & $8.88 \mathrm{E}-12$ & $6.22 \mathrm{E}-12$ & $2.53 \mathrm{E}-12$ & $1.70 \mathrm{E}-13$ & $2.81 \mathrm{E}-14$ & $2.96 \mathrm{E}-15$ & $9.00 \mathrm{E}-19$ \\
\hline OS191M & $1.37 \mathrm{E}-11$ & $3.82 \mathrm{E}-12$ & $1.06 \mathrm{E}-12$ & $3.80 \mathrm{E}-17$ & $2.92 \mathrm{E}-28$ & $0.00 \mathrm{E}+00$ & $0.00 \mathrm{E}+00$ & $0.00 \mathrm{E}+00$ & $0.00 \mathrm{E}+00$ \\
\hline IR192 & $2.21 \mathrm{E}-13$ & $2.19 \mathrm{E}-13$ & $2.17 \mathrm{E}-13$ & $2.02 \mathrm{E}-13$ & $1.67 \mathrm{E}-13$ & $9.53 \mathrm{E}-14$ & $6.55 \mathrm{E}-14$ & $4.10 \mathrm{E}-14$ & $7.60 \mathrm{E}-15$ \\
\hline IR194 & $9.27 \mathrm{E}-15$ & $3.89 \mathrm{E}-15$ & $1.64 \mathrm{E}-15$ & $1.58 \mathrm{E}-18$ & $4.49 \mathrm{E}-26$ & $0.00 \mathrm{E}+00$ & $0.00 \mathrm{E}+00$ & $0.00 \mathrm{E}+00$ & $0.00 \mathrm{E}+00$ \\
\hline IR194M & $4.89 \mathrm{E}-16$ & $0.00 \mathrm{E}+00$ & $0.00 \mathrm{E}+00$ & $0.00 \mathrm{E}+00$ & $0.00 \mathrm{E}+00$ & $0.00 \mathrm{E}+00$ & $0.00 \mathrm{E}+00$ & $0.00 \mathrm{E}+00$ & $0.00 \mathrm{E}+00$ \\
\hline
\end{tabular}


BSU-8242 3 DPA As-Run Physics Analysis

\begin{tabular}{|c|c|c|c|c|c|c|c|c|c|}
\hline \multicolumn{10}{|c|}{ ACTIVATION PRODUCTS (Ci) } \\
\hline ISOTOPE & EOC & 1 Day & 2 Days & 10 Days & 30 Days & 90 Days & 130 Days & 180 Days & 360 Days \\
\hline PT193M & $1.33 \mathrm{E}-16$ & $1.11 \mathrm{E}-16$ & $9.33 \mathrm{E}-17$ & $2.80 \mathrm{E}-17$ & $3.29 \mathrm{E}-18$ & $2.08 \mathrm{E}-22$ & $2.08 \mathrm{E}-22$ & $6.56 \mathrm{E}-26$ & $0.00 \mathrm{E}+00$ \\
\hline SUMTOT & $1.24 \mathrm{E}+04$ & $7.26 \mathrm{E}+03$ & $6.63 \mathrm{E}+03$ & $3.66 \mathrm{E}+03$ & $1.77 \mathrm{E}+03$ & $7.86 \mathrm{E}+02$ & $5.68 \mathrm{E}+02$ & $4.30 \mathrm{E}+02$ & $2.43 \mathrm{E}+02$ \\
\hline 0TOTAL & $1.24 \mathrm{E}+04$ & $7.26 \mathrm{E}+03$ & $6.63 \mathrm{E}+03$ & $3.66 \mathrm{E}+03$ & $1.77 \mathrm{E}+03$ & $7.86 \mathrm{E}+02$ & $5.68 \mathrm{E}+02$ & $4.30 \mathrm{E}+02$ & $2.43 \mathrm{E}+02$ \\
\hline
\end{tabular}

Table 21. Source Terms for 'A8 400C 3 DPA X' Capsule.

\begin{tabular}{|c|c|c|c|c|c|c|c|c|c|}
\hline \multicolumn{10}{|c|}{ ACTIVATION PRODUCTS } \\
\hline ISOTOPE & EOC & 1 Day & 2 Days & 10 Days & 30 Days & 90 Days & 130 Days & 180 Days & 360 Days \\
\hline $\mathrm{H} 3$ & $1.04 \mathrm{E}-04$ & $1.04 \mathrm{E}-04$ & $1.04 \mathrm{E}-04$ & $1.03 \mathrm{E}-04$ & $1.03 \mathrm{E}-04$ & $1.02 \mathrm{E}-04$ & $1.02 \mathrm{E}-04$ & $1.01 \mathrm{E}-04$ & $9.80 \mathrm{E}-05$ \\
\hline $\mathrm{H} 4$ & $3.07 \mathrm{E}-11$ & $0.00 \mathrm{E}+00$ & $0.00 \mathrm{E}+00$ & $0.00 \mathrm{E}+00$ & $0.00 \mathrm{E}+00$ & $0.00 \mathrm{E}+00$ & $0.00 \mathrm{E}+00$ & $0.00 \mathrm{E}+00$ & $0.00 \mathrm{E}+00$ \\
\hline HE 6 & $2.11 \mathrm{E}-04$ & $0.00 \mathrm{E}+00$ & $0.00 \mathrm{E}+00$ & $0.00 \mathrm{E}+00$ & $0.00 \mathrm{E}+00$ & $0.00 \mathrm{E}+00$ & $0.00 \mathrm{E}+00$ & $0.00 \mathrm{E}+00$ & $0.00 \mathrm{E}+00$ \\
\hline LI 8 & 2.09E-08 & $0.00 \mathrm{E}+00$ & $0.00 \mathrm{E}+00$ & $0.00 \mathrm{E}+00$ & $0.00 \mathrm{E}+00$ & $0.00 \mathrm{E}+00$ & $0.00 \mathrm{E}+00$ & $0.00 \mathrm{E}+00$ & $0.00 \mathrm{E}+00$ \\
\hline BE 8 & $3.63 \mathrm{E}-04$ & $0.00 \mathrm{E}+00$ & $0.00 \mathrm{E}+00$ & $0.00 \mathrm{E}+00$ & $0.00 \mathrm{E}+00$ & $0.00 \mathrm{E}+00$ & $0.00 \mathrm{E}+00$ & $0.00 \mathrm{E}+00$ & $0.00 \mathrm{E}+00$ \\
\hline BE 10 & $1.85 \mathrm{E}-09$ & $1.85 \mathrm{E}-09$ & $1.85 \mathrm{E}-09$ & $1.85 \mathrm{E}-09$ & $1.85 \mathrm{E}-09$ & $1.85 \mathrm{E}-09$ & $1.85 \mathrm{E}-09$ & $1.85 \mathrm{E}-09$ & $1.85 \mathrm{E}-09$ \\
\hline BE 11 & $1.17 \mathrm{E}-08$ & $0.00 \mathrm{E}+00$ & $0.00 \mathrm{E}+00$ & $0.00 \mathrm{E}+00$ & $0.00 \mathrm{E}+00$ & $0.00 \mathrm{E}+00$ & $0.00 \mathrm{E}+00$ & $0.00 \mathrm{E}+00$ & $0.00 \mathrm{E}+00$ \\
\hline B 12 & $4.36 \mathrm{E}-06$ & $0.00 \mathrm{E}+00$ & $0.00 \mathrm{E}+00$ & $0.00 \mathrm{E}+00$ & $0.00 \mathrm{E}+00$ & $0.00 \mathrm{E}+00$ & $0.00 \mathrm{E}+00$ & $0.00 \mathrm{E}+00$ & $0.00 \mathrm{E}+00$ \\
\hline C 14 & $2.14 \mathrm{E}-04$ & $2.14 \mathrm{E}-04$ & $2.14 \mathrm{E}-04$ & $2.14 \mathrm{E}-04$ & $2.14 \mathrm{E}-04$ & $2.14 \mathrm{E}-04$ & $2.14 \mathrm{E}-04$ & $2.14 \mathrm{E}-04$ & $2.14 \mathrm{E}-04$ \\
\hline C 15 & $5.10 \mathrm{E}-08$ & $0.00 \mathrm{E}+00$ & $0.00 \mathrm{E}+00$ & $0.00 \mathrm{E}+00$ & $0.00 \mathrm{E}+00$ & $0.00 \mathrm{E}+00$ & $0.00 \mathrm{E}+00$ & $0.00 \mathrm{E}+00$ & $0.00 \mathrm{E}+00$ \\
\hline N 16 & $1.65 \mathrm{E}-07$ & $0.00 \mathrm{E}+00$ & $0.00 \mathrm{E}+00$ & $0.00 \mathrm{E}+00$ & $0.00 \mathrm{E}+00$ & $0.00 \mathrm{E}+00$ & $0.00 \mathrm{E}+00$ & $0.00 \mathrm{E}+00$ & $0.00 \mathrm{E}+00$ \\
\hline O 19 & $1.07 \mathrm{E}-10$ & $0.00 \mathrm{E}+00$ & $0.00 \mathrm{E}+00$ & $0.00 \mathrm{E}+00$ & $0.00 \mathrm{E}+00$ & $0.00 \mathrm{E}+00$ & $0.00 \mathrm{E}+00$ & $0.00 \mathrm{E}+00$ & $0.00 \mathrm{E}+00$ \\
\hline F 20 & $1.54 \mathrm{E}-07$ & $0.00 \mathrm{E}+00$ & $0.00 \mathrm{E}+00$ & $0.00 \mathrm{E}+00$ & $0.00 \mathrm{E}+00$ & $0.00 \mathrm{E}+00$ & $0.00 \mathrm{E}+00$ & $0.00 \mathrm{E}+00$ & $0.00 \mathrm{E}+00$ \\
\hline NE 23 & $6.14 \mathrm{E}-02$ & $0.00 \mathrm{E}+00$ & $0.00 \mathrm{E}+00$ & $0.00 \mathrm{E}+00$ & $0.00 \mathrm{E}+00$ & $0.00 \mathrm{E}+00$ & $0.00 \mathrm{E}+00$ & $0.00 \mathrm{E}+00$ & $0.00 \mathrm{E}+00$ \\
\hline NA 24 & $1.51 \mathrm{E}+01$ & $4.99 \mathrm{E}+00$ & $1.64 \mathrm{E}+00$ & $2.31 \mathrm{E}-04$ & $5.37 \mathrm{E}-14$ & $0.00 \mathrm{E}+00$ & $0.00 \mathrm{E}+00$ & $0.00 \mathrm{E}+00$ & $0.00 \mathrm{E}+00$ \\
\hline NA $24 \mathrm{M}$ & $3.42 \mathrm{E}-05$ & $0.00 \mathrm{E}+00$ & $0.00 \mathrm{E}+00$ & $0.00 \mathrm{E}+00$ & $0.00 \mathrm{E}+00$ & $0.00 \mathrm{E}+00$ & $0.00 \mathrm{E}+00$ & $0.00 \mathrm{E}+00$ & $0.00 \mathrm{E}+00$ \\
\hline NA 25 & $3.71 \mathrm{E}-02$ & $0.00 \mathrm{E}+00$ & $0.00 \mathrm{E}+00$ & $0.00 \mathrm{E}+00$ & $0.00 \mathrm{E}+00$ & $0.00 \mathrm{E}+00$ & $0.00 \mathrm{E}+00$ & $0.00 \mathrm{E}+00$ & $0.00 \mathrm{E}+00$ \\
\hline MG 27 & $7.16 \mathrm{E}+01$ & $0.00 \mathrm{E}+00$ & $0.00 \mathrm{E}+00$ & $0.00 \mathrm{E}+00$ & $0.00 \mathrm{E}+00$ & $0.00 \mathrm{E}+00$ & $0.00 \mathrm{E}+00$ & $0.00 \mathrm{E}+00$ & $0.00 \mathrm{E}+00$ \\
\hline MG 28 & $2.03 \mathrm{E}-07$ & $9.14 \mathrm{E}-08$ & 4.13E-08 & $7.11 \mathrm{E}-11$ & $8.75 \mathrm{E}-18$ & $0.00 \mathrm{E}+00$ & $0.00 \mathrm{E}+00$ & $0.00 \mathrm{E}+00$ & $0.00 \mathrm{E}+00$ \\
\hline
\end{tabular}


BSU-8242 3 DPA As-Run Physics Analysis

\begin{tabular}{|c|c|c|c|c|c|c|c|c|c|}
\hline \multicolumn{10}{|c|}{ ACTIVATION PRODUCTS } \\
\hline ISOTOPE & EOC & 1 Day & 2 Days & 10 Days & 30 Days & 90 Days & 130 Days & 180 Days & 360 Days \\
\hline AL 28 & $1.71 \mathrm{E}+03$ & $9.16 \mathrm{E}-08$ & 4.13E-08 & $7.12 \mathrm{E}-11$ & $8.77 \mathrm{E}-18$ & $0.00 \mathrm{E}+00$ & $0.00 \mathrm{E}+00$ & $0.00 \mathrm{E}+00$ & $0.00 \mathrm{E}+00$ \\
\hline AL 29 & $3.50 \mathrm{E}-02$ & $0.00 \mathrm{E}+00$ & $0.00 \mathrm{E}+00$ & $0.00 \mathrm{E}+00$ & $0.00 \mathrm{E}+00$ & $0.00 \mathrm{E}+00$ & $0.00 \mathrm{E}+00$ & $0.00 \mathrm{E}+00$ & $0.00 \mathrm{E}+00$ \\
\hline AL 30 & $3.38 \mathrm{E}-05$ & $0.00 \mathrm{E}+00$ & $0.00 \mathrm{E}+00$ & $0.00 \mathrm{E}+00$ & $0.00 \mathrm{E}+00$ & $0.00 \mathrm{E}+00$ & $0.00 \mathrm{E}+00$ & $0.00 \mathrm{E}+00$ & $0.00 \mathrm{E}+00$ \\
\hline SI 31 & $3.84 \mathrm{E}-01$ & $6.74 \mathrm{E}-04$ & $1.18 \mathrm{E}-06$ & $0.00 \mathrm{E}+00$ & $0.00 \mathrm{E}+00$ & $0.00 \mathrm{E}+00$ & $0.00 \mathrm{E}+00$ & $0.00 \mathrm{E}+00$ & $0.00 \mathrm{E}+00$ \\
\hline SI 32 & $1.09 \mathrm{E}-10$ & $1.09 \mathrm{E}-10$ & $1.09 \mathrm{E}-10$ & $1.09 \mathrm{E}-10$ & $1.09 \mathrm{E}-10$ & $1.09 \mathrm{E}-10$ & $1.09 \mathrm{E}-10$ & $1.09 \mathrm{E}-10$ & $1.09 \mathrm{E}-10$ \\
\hline P 32 & $2.86 \mathrm{E}-01$ & $2.72 \mathrm{E}-01$ & $2.59 \mathrm{E}-01$ & $1.76 \mathrm{E}-01$ & $6.67 \mathrm{E}-02$ & $3.64 \mathrm{E}-03$ & $5.24 \mathrm{E}-04$ & 4.64E-05 & 7.65E-09 \\
\hline P 33 & $1.33 \mathrm{E}-05$ & $1.29 \mathrm{E}-05$ & $1.26 \mathrm{E}-05$ & $1.01 \mathrm{E}-05$ & $5.79 \mathrm{E}-06$ & $1.10 \mathrm{E}-06$ & $3.62 \mathrm{E}-07$ & $9.04 \mathrm{E}-08$ & $6.15 \mathrm{E}-10$ \\
\hline P 34 & $8.31 \mathrm{E}-05$ & $0.00 \mathrm{E}+00$ & $0.00 \mathrm{E}+00$ & $0.00 \mathrm{E}+00$ & $0.00 \mathrm{E}+00$ & $0.00 \mathrm{E}+00$ & $0.00 \mathrm{E}+00$ & $0.00 \mathrm{E}+00$ & $0.00 \mathrm{E}+00$ \\
\hline S 35 & $5.59 \mathrm{E}-03$ & $5.54 \mathrm{E}-03$ & $5.50 \mathrm{E}-03$ & $5.16 \mathrm{E}-03$ & $4.41 \mathrm{E}-03$ & $2.75 \mathrm{E}-03$ & $2.01 \mathrm{E}-03$ & $1.35 \mathrm{E}-03$ & $3.28 \mathrm{E}-04$ \\
\hline S 37 & $2.40 \mathrm{E}-05$ & $0.00 \mathrm{E}+00$ & $0.00 \mathrm{E}+00$ & $0.00 \mathrm{E}+00$ & $0.00 \mathrm{E}+00$ & $0.00 \mathrm{E}+00$ & $0.00 \mathrm{E}+00$ & $0.00 \mathrm{E}+00$ & $0.00 \mathrm{E}+00$ \\
\hline CL 36 & $1.68 \mathrm{E}-10$ & $1.68 \mathrm{E}-10$ & $1.68 \mathrm{E}-10$ & $1.68 \mathrm{E}-10$ & $1.68 \mathrm{E}-10$ & $1.68 \mathrm{E}-10$ & $1.68 \mathrm{E}-10$ & $1.68 \mathrm{E}-10$ & $1.68 \mathrm{E}-10$ \\
\hline CL 38 & $1.52 \mathrm{E}-08$ & $3.42 \mathrm{E}-20$ & $6.85 \mathrm{E}-32$ & $0.00 \mathrm{E}+00$ & $0.00 \mathrm{E}+00$ & $0.00 \mathrm{E}+00$ & $0.00 \mathrm{E}+00$ & $0.00 \mathrm{E}+00$ & $0.00 \mathrm{E}+00$ \\
\hline CL 38M & $1.81 \mathrm{E}-10$ & $0.00 \mathrm{E}+00$ & $0.00 \mathrm{E}+00$ & $0.00 \mathrm{E}+00$ & $0.00 \mathrm{E}+00$ & $0.00 \mathrm{E}+00$ & $0.00 \mathrm{E}+00$ & $0.00 \mathrm{E}+00$ & $0.00 \mathrm{E}+00$ \\
\hline AR 37 & $2.43 \mathrm{E}-13$ & $2.38 \mathrm{E}-13$ & $2.33 \mathrm{E}-13$ & $1.99 \mathrm{E}-13$ & $1.34 \mathrm{E}-13$ & $4.09 \mathrm{E}-14$ & $1.85 \mathrm{E}-14$ & $6.88 \mathrm{E}-15$ & $1.95 \mathrm{E}-16$ \\
\hline AR 39 & $2.85 \mathrm{E}-15$ & $2.85 \mathrm{E}-15$ & $2.85 \mathrm{E}-15$ & $2.85 \mathrm{E}-15$ & $2.85 \mathrm{E}-15$ & $2.85 \mathrm{E}-15$ & $2.85 \mathrm{E}-15$ & $2.85 \mathrm{E}-15$ & $2.84 \mathrm{E}-15$ \\
\hline AR 41 & $2.47 \mathrm{E}-09$ & $2.74 \mathrm{E}-13$ & $3.04 \mathrm{E}-17$ & $0.00 \mathrm{E}+00$ & $0.00 \mathrm{E}+00$ & $0.00 \mathrm{E}+00$ & $0.00 \mathrm{E}+00$ & $0.00 \mathrm{E}+00$ & $0.00 \mathrm{E}+00$ \\
\hline AR 42 & $5.11 \mathrm{E}-18$ & $5.11 \mathrm{E}-18$ & $5.11 \mathrm{E}-18$ & $5.10 \mathrm{E}-18$ & $5.10 \mathrm{E}-18$ & $5.08 \mathrm{E}-18$ & $5.07 \mathrm{E}-18$ & $5.05 \mathrm{E}-18$ & $5.00 \mathrm{E}-18$ \\
\hline K 42 & $2.52 \mathrm{E}-11$ & $6.57 \mathrm{E}-12$ & $1.71 \mathrm{E}-12$ & 4.12E-17 & $5.10 \mathrm{E}-18$ & $5.08 \mathrm{E}-18$ & $5.07 \mathrm{E}-18$ & $5.05 \mathrm{E}-18$ & $5.00 \mathrm{E}-18$ \\
\hline K 43 & $3.13 \mathrm{E}-08$ & $1.50 \mathrm{E}-08$ & $7.18 \mathrm{E}-09$ & $1.99 \mathrm{E}-11$ & 8.03E-18 & $0.00 \mathrm{E}+00$ & $0.00 \mathrm{E}+00$ & $0.00 \mathrm{E}+00$ & $0.00 \mathrm{E}+00$ \\
\hline K 44 & $5.56 \mathrm{E}-09$ & $1.10 \mathrm{E}-28$ & $0.00 \mathrm{E}+00$ & $0.00 \mathrm{E}+00$ & $0.00 \mathrm{E}+00$ & $0.00 \mathrm{E}+00$ & $0.00 \mathrm{E}+00$ & $0.00 \mathrm{E}+00$ & $0.00 \mathrm{E}+00$ \\
\hline CA 45 & $1.38 \mathrm{E}-04$ & $1.38 \mathrm{E}-04$ & $1.37 \mathrm{E}-04$ & $1.33 \mathrm{E}-04$ & $1.22 \mathrm{E}-04$ & $9.44 \mathrm{E}-05$ & $7.96 \mathrm{E}-05$ & $6.43 \mathrm{E}-05$ & $2.99 \mathrm{E}-05$ \\
\hline CA 47 & $2.47 \mathrm{E}-06$ & $2.12 \mathrm{E}-06$ & $1.82 \mathrm{E}-06$ & $5.37 \mathrm{E}-07$ & $2.53 \mathrm{E}-08$ & $2.63 \mathrm{E}-12$ & $5.84 \mathrm{E}-15$ & $2.81 \mathrm{E}-18$ & $3.17 \mathrm{E}-30$ \\
\hline $\mathrm{SC} 46$ & $2.40 \mathrm{E}-02$ & $2.38 \mathrm{E}-02$ & $2.36 \mathrm{E}-02$ & $2.21 \mathrm{E}-02$ & $1.87 \mathrm{E}-02$ & $1.14 \mathrm{E}-02$ & $8.18 \mathrm{E}-03$ & $5.41 \mathrm{E}-03$ & $1.22 \mathrm{E}-03$ \\
\hline $\mathrm{SC} 46 \mathrm{M}$ & $1.73 \mathrm{E}-06$ & $0.00 \mathrm{E}+00$ & $0.00 \mathrm{E}+00$ & $0.00 \mathrm{E}+00$ & $0.00 \mathrm{E}+00$ & $0.00 \mathrm{E}+00$ & $0.00 \mathrm{E}+00$ & $0.00 \mathrm{E}+00$ & $0.00 \mathrm{E}+00$ \\
\hline SC 47 & $6.22 \mathrm{E}-02$ & $5.06 \mathrm{E}-02$ & 4.11E-02 & $7.86 \mathrm{E}-03$ & $1.26 \mathrm{E}-04$ & $5.21 \mathrm{E}-10$ & $1.55 \mathrm{E}-13$ & $1.50 \mathrm{E}-17$ & $1.21 \mathrm{E}-29$ \\
\hline $\mathrm{SC} 48$ & $5.87 \mathrm{E}-03$ & $4.02 \mathrm{E}-03$ & $2.75 \mathrm{E}-03$ & $1.32 \mathrm{E}-04$ & $6.62 \mathrm{E}-08$ & $8.42 \mathrm{E}-18$ & $2.13 \mathrm{E}-24$ & $2.28 \mathrm{E}-32$ & $0.00 \mathrm{E}+00$ \\
\hline
\end{tabular}


BSU-8242 3 DPA As-Run Physics Analysis

\begin{tabular}{|c|c|c|c|c|c|c|c|c|c|}
\hline \multicolumn{10}{|c|}{ ACTIVATION PRODUCTS } \\
\hline ISOTOPE & EOC & 1 Day & 2 Days & 10 Days & 30 Days & 90 Days & 130 Days & 180 Days & 360 Days \\
\hline SC 49 & $5.38 \mathrm{E}-03$ & $1.55 \mathrm{E}-10$ & $4.50 \mathrm{E}-18$ & $0.00 \mathrm{E}+00$ & $0.00 \mathrm{E}+00$ & $0.00 \mathrm{E}+00$ & $0.00 \mathrm{E}+00$ & $0.00 \mathrm{E}+00$ & $0.00 \mathrm{E}+00$ \\
\hline SC 50 & $9.96 \mathrm{E}-05$ & $0.00 \mathrm{E}+00$ & $0.00 \mathrm{E}+00$ & $0.00 \mathrm{E}+00$ & $0.00 \mathrm{E}+00$ & $0.00 \mathrm{E}+00$ & $0.00 \mathrm{E}+00$ & $0.00 \mathrm{E}+00$ & $0.00 \mathrm{E}+00$ \\
\hline TI 51 & $1.31 \mathrm{E}-01$ & $0.00 \mathrm{E}+00$ & $0.00 \mathrm{E}+00$ & $0.00 \mathrm{E}+00$ & $0.00 \mathrm{E}+00$ & $0.00 \mathrm{E}+00$ & $0.00 \mathrm{E}+00$ & $0.00 \mathrm{E}+00$ & $0.00 \mathrm{E}+00$ \\
\hline V 50 & $1.63 \mathrm{E}-16$ & $1.63 \mathrm{E}-16$ & $1.63 \mathrm{E}-16$ & $1.63 \mathrm{E}-16$ & $1.63 \mathrm{E}-16$ & $1.63 \mathrm{E}-16$ & $1.63 \mathrm{E}-16$ & $1.63 \mathrm{E}-16$ & $1.63 \mathrm{E}-16$ \\
\hline V 52 & $4.80 \mathrm{E}+01$ & $0.00 \mathrm{E}+00$ & $0.00 \mathrm{E}+00$ & $0.00 \mathrm{E}+00$ & $0.00 \mathrm{E}+00$ & $0.00 \mathrm{E}+00$ & $0.00 \mathrm{E}+00$ & $0.00 \mathrm{E}+00$ & $0.00 \mathrm{E}+00$ \\
\hline V 53 & $1.24 \mathrm{E}-01$ & $0.00 \mathrm{E}+00$ & $0.00 \mathrm{E}+00$ & $0.00 \mathrm{E}+00$ & $0.00 \mathrm{E}+00$ & $0.00 \mathrm{E}+00$ & $0.00 \mathrm{E}+00$ & $0.00 \mathrm{E}+00$ & $0.00 \mathrm{E}+00$ \\
\hline V 54 & $1.35 \mathrm{E}-03$ & $0.00 \mathrm{E}+00$ & $0.00 \mathrm{E}+00$ & $0.00 \mathrm{E}+00$ & $0.00 \mathrm{E}+00$ & $0.00 \mathrm{E}+00$ & $0.00 \mathrm{E}+00$ & $0.00 \mathrm{E}+00$ & $0.00 \mathrm{E}+00$ \\
\hline CR 51 & $2.28 \mathrm{E}+03$ & $2.23 \mathrm{E}+03$ & $2.17 \mathrm{E}+03$ & $1.78 \mathrm{E}+03$ & $1.08 \mathrm{E}+03$ & $2.40 \mathrm{E}+02$ & $8.83 \mathrm{E}+01$ & $2.53 \mathrm{E}+01$ & $2.80 \mathrm{E}-01$ \\
\hline CR 55 & $3.70 \mathrm{E}+01$ & $0.00 \mathrm{E}+00$ & $0.00 \mathrm{E}+00$ & $0.00 \mathrm{E}+00$ & $0.00 \mathrm{E}+00$ & $0.00 \mathrm{E}+00$ & $0.00 \mathrm{E}+00$ & $0.00 \mathrm{E}+00$ & $0.00 \mathrm{E}+00$ \\
\hline MN 54 & $3.17 \mathrm{E}+01$ & $3.16 \mathrm{E}+01$ & $3.15 \mathrm{E}+01$ & $3.10 \mathrm{E}+01$ & $2.96 \mathrm{E}+01$ & $2.59 \mathrm{E}+01$ & $2.37 \mathrm{E}+01$ & $2.12 \mathrm{E}+01$ & $1.42 \mathrm{E}+01$ \\
\hline MN 56 & $8.69 \mathrm{E}+02$ & $1.37 \mathrm{E}+00$ & $2.16 \mathrm{E}-03$ & $0.00 \mathrm{E}+00$ & $0.00 \mathrm{E}+00$ & $0.00 \mathrm{E}+00$ & $0.00 \mathrm{E}+00$ & $0.00 \mathrm{E}+00$ & $0.00 \mathrm{E}+00$ \\
\hline MN 57 & $2.92 \mathrm{E}-01$ & $0.00 \mathrm{E}+00$ & $0.00 \mathrm{E}+00$ & $0.00 \mathrm{E}+00$ & $0.00 \mathrm{E}+00$ & $0.00 \mathrm{E}+00$ & $0.00 \mathrm{E}+00$ & $0.00 \mathrm{E}+00$ & $0.00 \mathrm{E}+00$ \\
\hline MN 58 & $8.78 \mathrm{E}-04$ & $0.00 \mathrm{E}+00$ & $0.00 \mathrm{E}+00$ & $0.00 \mathrm{E}+00$ & $0.00 \mathrm{E}+00$ & $0.00 \mathrm{E}+00$ & $0.00 \mathrm{E}+00$ & $0.00 \mathrm{E}+00$ & $0.00 \mathrm{E}+00$ \\
\hline FE 55 & $1.42 \mathrm{E}+02$ & $1.42 \mathrm{E}+02$ & $1.42 \mathrm{E}+02$ & $1.41 \mathrm{E}+02$ & $1.39 \mathrm{E}+02$ & $1.33 \mathrm{E}+02$ & $1.29 \mathrm{E}+02$ & $1.24 \mathrm{E}+02$ & $1.09 \mathrm{E}+02$ \\
\hline FE 59 & $2.63 \mathrm{E}+01$ & $2.59 \mathrm{E}+01$ & $2.55 \mathrm{E}+01$ & $2.25 \mathrm{E}+01$ & $1.66 \mathrm{E}+01$ & $6.57 \mathrm{E}+00$ & $3.55 \mathrm{E}+00$ & $1.64 \mathrm{E}+00$ & $1.03 \mathrm{E}-01$ \\
\hline CO 58 & $2.10 \mathrm{E}+02$ & $2.08 \mathrm{E}+02$ & $2.06 \mathrm{E}+02$ & $1.91 \mathrm{E}+02$ & $1.57 \mathrm{E}+02$ & $8.70 \mathrm{E}+01$ & $5.88 \mathrm{E}+01$ & $3.60 \mathrm{E}+01$ & $6.18 \mathrm{E}+00$ \\
\hline CO 60 & $1.16 \mathrm{E}+02$ & $1.16 \mathrm{E}+02$ & $1.16 \mathrm{E}+02$ & $1.16 \mathrm{E}+02$ & $1.15 \mathrm{E}+02$ & $1.12 \mathrm{E}+02$ & $1.11 \mathrm{E}+02$ & $1.09 \mathrm{E}+02$ & $1.02 \mathrm{E}+02$ \\
\hline $\mathrm{CO} 60 \mathrm{M}$ & $9.98 \mathrm{E}+02$ & $0.00 \mathrm{E}+00$ & $0.00 \mathrm{E}+00$ & $0.00 \mathrm{E}+00$ & $0.00 \mathrm{E}+00$ & $0.00 \mathrm{E}+00$ & $0.00 \mathrm{E}+00$ & $0.00 \mathrm{E}+00$ & $0.00 \mathrm{E}+00$ \\
\hline $\mathrm{CO} 61$ & $7.35 \mathrm{E}+00$ & $3.08 \mathrm{E}-04$ & $1.29 \mathrm{E}-08$ & $0.00 \mathrm{E}+00$ & $0.00 \mathrm{E}+00$ & $0.00 \mathrm{E}+00$ & $0.00 \mathrm{E}+00$ & $0.00 \mathrm{E}+00$ & $0.00 \mathrm{E}+00$ \\
\hline $\mathrm{CO} 62$ & $6.49 \mathrm{E}-02$ & $0.00 \mathrm{E}+00$ & $0.00 \mathrm{E}+00$ & $0.00 \mathrm{E}+00$ & $0.00 \mathrm{E}+00$ & $0.00 \mathrm{E}+00$ & $0.00 \mathrm{E}+00$ & $0.00 \mathrm{E}+00$ & $0.00 \mathrm{E}+00$ \\
\hline NI 59 & $7.31 \mathrm{E}-02$ & 7.31E-02 & $7.31 \mathrm{E}-02$ & $7.31 \mathrm{E}-02$ & $7.31 \mathrm{E}-02$ & $7.31 \mathrm{E}-02$ & $7.31 \mathrm{E}-02$ & $7.31 \mathrm{E}-02$ & $7.31 \mathrm{E}-02$ \\
\hline NI 63 & $9.62 \mathrm{E}+00$ & $9.62 \mathrm{E}+00$ & $9.62 \mathrm{E}+00$ & $9.62 \mathrm{E}+00$ & $9.61 \mathrm{E}+00$ & $9.60 \mathrm{E}+00$ & $9.59 \mathrm{E}+00$ & $9.58 \mathrm{E}+00$ & $9.55 \mathrm{E}+00$ \\
\hline NI 65 & $7.57 \mathrm{E}+01$ & $1.03 \mathrm{E}-01$ & $1.39 \mathrm{E}-04$ & $0.00 \mathrm{E}+00$ & $0.00 \mathrm{E}+00$ & $0.00 \mathrm{E}+00$ & $0.00 \mathrm{E}+00$ & $0.00 \mathrm{E}+00$ & $0.00 \mathrm{E}+00$ \\
\hline NI 66 & $2.08 \mathrm{E}-03$ & $1.53 \mathrm{E}-03$ & $1.13 \mathrm{E}-03$ & $9.89 \mathrm{E}-05$ & $2.23 \mathrm{E}-07$ & $2.58 \mathrm{E}-15$ & $1.32 \mathrm{E}-20$ & $3.20 \mathrm{E}-27$ & $0.00 \mathrm{E}+00$ \\
\hline CU 64 & $9.39 \mathrm{E}+01$ & $2.53 \mathrm{E}+01$ & $6.84 \mathrm{E}+00$ & $1.92 \mathrm{E}-04$ & $8.06 \mathrm{E}-16$ & $0.00 \mathrm{E}+00$ & $0.00 \mathrm{E}+00$ & $0.00 \mathrm{E}+00$ & $0.00 \mathrm{E}+00$ \\
\hline CU 66 & $2.06 \mathrm{E}+01$ & $1.54 \mathrm{E}-03$ & $1.13 \mathrm{E}-03$ & $9.90 \mathrm{E}-05$ & $2.24 \mathrm{E}-07$ & $2.58 \mathrm{E}-15$ & $1.32 \mathrm{E}-20$ & $3.20 \mathrm{E}-27$ & $0.00 \mathrm{E}+00$ \\
\hline
\end{tabular}


BSU-8242 3 DPA As-Run Physics Analysis

\begin{tabular}{|c|c|c|c|c|c|c|c|c|c|}
\hline \multicolumn{10}{|c|}{ ACTIVATION PRODUCTS } \\
\hline ISOTOPE & EOC & 1 Day & 2 Days & 10 Days & 30 Days & 90 Days & 130 Days & 180 Days & 360 Days \\
\hline CU 67 & $1.06 \mathrm{E}-04$ & 8.09E-05 & $6.18 \mathrm{E}-05$ & 7.19E-06 & 3.32E-08 & $3.26 \mathrm{E}-15$ & $6.95 \mathrm{E}-20$ & $1.01 \mathrm{E}-25$ & $0.00 \mathrm{E}+00$ \\
\hline ZN 65 & $1.01 \mathrm{E}-02$ & $1.01 \mathrm{E}-02$ & $1.01 \mathrm{E}-02$ & $9.84 \mathrm{E}-03$ & $9.29 \mathrm{E}-03$ & $7.84 \mathrm{E}-03$ & $7.00 \mathrm{E}-03$ & $6.07 \mathrm{E}-03$ & $3.64 \mathrm{E}-03$ \\
\hline ZN 69 & $1.67 \mathrm{E}-07$ & 3.59E-09 & $1.07 \mathrm{E}-09$ & $6.77 \mathrm{E}-14$ & $2.14 \mathrm{E}-24$ & $0.00 \mathrm{E}+00$ & $0.00 \mathrm{E}+00$ & $0.00 \mathrm{E}+00$ & $0.00 \mathrm{E}+00$ \\
\hline ZN 69M & $1.12 \mathrm{E}-08$ & $3.35 \mathrm{E}-09$ & $9.99 \mathrm{E}-10$ & $6.30 \mathrm{E}-14$ & $1.99 \mathrm{E}-24$ & $0.00 \mathrm{E}+00$ & $0.00 \mathrm{E}+00$ & $0.00 \mathrm{E}+00$ & $0.00 \mathrm{E}+00$ \\
\hline GA 70 & $3.17 \mathrm{E}-10$ & $9.13 \mathrm{E}-31$ & $0.00 \mathrm{E}+00$ & $0.00 \mathrm{E}+00$ & $0.00 \mathrm{E}+00$ & $0.00 \mathrm{E}+00$ & $0.00 \mathrm{E}+00$ & $0.00 \mathrm{E}+00$ & $0.00 \mathrm{E}+00$ \\
\hline GA 72 & $5.56 \mathrm{E}-16$ & $1.80 \mathrm{E}-16$ & $4.35 \mathrm{E}-17$ & $3.47 \mathrm{E}-21$ & $2.05 \mathrm{E}-31$ & $0.00 \mathrm{E}+00$ & $0.00 \mathrm{E}+00$ & $0.00 \mathrm{E}+00$ & $0.00 \mathrm{E}+00$ \\
\hline GE 71 & $2.08 \mathrm{E}-13$ & $1.96 \mathrm{E}-13$ & $1.85 \mathrm{E}-13$ & $1.16 \mathrm{E}-13$ & $3.57 \mathrm{E}-14$ & $1.05 \mathrm{E}-15$ & $1.01 \mathrm{E}-16$ & $5.62 \mathrm{E}-18$ & $1.44 \mathrm{E}-22$ \\
\hline GE $71 \mathrm{M}$ & $2.56 \mathrm{E}-14$ & $0.00 \mathrm{E}+00$ & $0.00 \mathrm{E}+00$ & $0.00 \mathrm{E}+00$ & $0.00 \mathrm{E}+00$ & $0.00 \mathrm{E}+00$ & $0.00 \mathrm{E}+00$ & $0.00 \mathrm{E}+00$ & $0.00 \mathrm{E}+00$ \\
\hline SR 89 & $1.34 \mathrm{E}-07$ & $1.32 \mathrm{E}-07$ & $1.31 \mathrm{E}-07$ & $1.17 \mathrm{E}-07$ & 8.88E-08 & $3.90 \mathrm{E}-08$ & $2.25 \mathrm{E}-08$ & $1.13 \mathrm{E}-08$ & $9.58 \mathrm{E}-10$ \\
\hline SR 90 & $1.64 \mathrm{E}-10$ & $1.64 \mathrm{E}-10$ & $1.64 \mathrm{E}-10$ & $1.64 \mathrm{E}-10$ & $1.64 \mathrm{E}-10$ & $1.63 \mathrm{E}-10$ & $1.63 \mathrm{E}-10$ & $1.62 \mathrm{E}-10$ & $1.60 \mathrm{E}-10$ \\
\hline SR 91 & $4.06 \mathrm{E}-09$ & $7.05 \mathrm{E}-10$ & $1.22 \mathrm{E}-10$ & $1.01 \mathrm{E}-16$ & $6.85 \mathrm{E}-32$ & $0.00 \mathrm{E}+00$ & $0.00 \mathrm{E}+00$ & $0.00 \mathrm{E}+00$ & $0.00 \mathrm{E}+00$ \\
\hline SR 93 & $6.93 \mathrm{E}-15$ & $0.00 \mathrm{E}+00$ & $0.00 \mathrm{E}+00$ & $0.00 \mathrm{E}+00$ & $0.00 \mathrm{E}+00$ & $0.00 \mathrm{E}+00$ & $0.00 \mathrm{E}+00$ & $0.00 \mathrm{E}+00$ & $0.00 \mathrm{E}+00$ \\
\hline Y 89M & $2.34 \mathrm{E}-03$ & $1.89 \mathrm{E}-03$ & $1.53 \mathrm{E}-03$ & $2.80 \mathrm{E}-04$ & $4.03 \mathrm{E}-06$ & $1.20 \mathrm{E}-11$ & $2.49 \mathrm{E}-15$ & $6.17 \mathrm{E}-20$ & $0.00 \mathrm{E}+00$ \\
\hline Y 90 & $1.06 \mathrm{E}-02$ & $8.17 \mathrm{E}-03$ & $6.30 \mathrm{E}-03$ & $7.88 \mathrm{E}-04$ & $4.35 \mathrm{E}-06$ & $1.64 \mathrm{E}-10$ & $1.63 \mathrm{E}-10$ & $1.62 \mathrm{E}-10$ & $1.61 \mathrm{E}-10$ \\
\hline Y 90M & 3.33E-09 & $1.56 \mathrm{E}-11$ & 7.27E-14 & $2.28 \mathrm{E}-32$ & $0.00 \mathrm{E}+00$ & $0.00 \mathrm{E}+00$ & $0.00 \mathrm{E}+00$ & $0.00 \mathrm{E}+00$ & $0.00 \mathrm{E}+00$ \\
\hline Y 91 & $9.48 \mathrm{E}-07$ & $9.37 \mathrm{E}-07$ & $9.26 \mathrm{E}-07$ & $8.42 \mathrm{E}-07$ & $6.64 \mathrm{E}-07$ & $3.26 \mathrm{E}-07$ & $2.03 \mathrm{E}-07$ & $1.12 \mathrm{E}-07$ & $1.33 \mathrm{E}-08$ \\
\hline Y 92 & $5.16 \mathrm{E}-07$ & 4.69E-09 & $4.26 \mathrm{E}-11$ & $1.98 \mathrm{E}-27$ & $0.00 \mathrm{E}+00$ & $0.00 \mathrm{E}+00$ & $0.00 \mathrm{E}+00$ & $0.00 \mathrm{E}+00$ & $0.00 \mathrm{E}+00$ \\
\hline Y 93 & $6.93 \mathrm{E}-15$ & $1.34 \mathrm{E}-15$ & $2.66 \mathrm{E}-16$ & $5.04 \mathrm{E}-22$ & $0.00 \mathrm{E}+00$ & $0.00 \mathrm{E}+00$ & $0.00 \mathrm{E}+00$ & $0.00 \mathrm{E}+00$ & $0.00 \mathrm{E}+00$ \\
\hline Y 94 & 2.89E-09 & $0.00 \mathrm{E}+00$ & $0.00 \mathrm{E}+00$ & $0.00 \mathrm{E}+00$ & $0.00 \mathrm{E}+00$ & $0.00 \mathrm{E}+00$ & $0.00 \mathrm{E}+00$ & $0.00 \mathrm{E}+00$ & $0.00 \mathrm{E}+00$ \\
\hline Y 96 & $9.22 \mathrm{E}-15$ & $0.00 \mathrm{E}+00$ & $0.00 \mathrm{E}+00$ & $0.00 \mathrm{E}+00$ & $0.00 \mathrm{E}+00$ & $0.00 \mathrm{E}+00$ & $0.00 \mathrm{E}+00$ & $0.00 \mathrm{E}+00$ & $0.00 \mathrm{E}+00$ \\
\hline ZR 89 & $2.34 \mathrm{E}-03$ & $1.89 \mathrm{E}-03$ & $1.53 \mathrm{E}-03$ & $2.81 \mathrm{E}-04$ & $4.04 \mathrm{E}-06$ & $1.20 \mathrm{E}-11$ & $2.49 \mathrm{E}-15$ & $6.18 \mathrm{E}-20$ & $0.00 \mathrm{E}+00$ \\
\hline ZR 93 & $2.80 \mathrm{E}-08$ & $2.80 \mathrm{E}-08$ & $2.80 \mathrm{E}-08$ & $2.80 \mathrm{E}-08$ & $2.80 \mathrm{E}-08$ & $2.80 \mathrm{E}-08$ & $2.80 \mathrm{E}-08$ & $2.80 \mathrm{E}-08$ & $2.80 \mathrm{E}-08$ \\
\hline ZR 95 & $5.41 \mathrm{E}-03$ & $5.35 \mathrm{E}-03$ & $5.29 \mathrm{E}-03$ & $4.85 \mathrm{E}-03$ & $3.91 \mathrm{E}-03$ & $2.04 \mathrm{E}-03$ & $1.32 \mathrm{E}-03$ & 7.69E-04 & $1.09 \mathrm{E}-04$ \\
\hline ZR 97 & $1.85 \mathrm{E}-04$ & $6.90 \mathrm{E}-05$ & $2.58 \mathrm{E}-05$ & $9.81 \mathrm{E}-09$ & $2.77 \mathrm{E}-17$ & $0.00 \mathrm{E}+00$ & $0.00 \mathrm{E}+00$ & $0.00 \mathrm{E}+00$ & $0.00 \mathrm{E}+00$ \\
\hline NB 92 & $4.59 \mathrm{E}-01$ & 4.29E-01 & $4.00 \mathrm{E}-01$ & $2.32 \mathrm{E}-01$ & $5.93 \mathrm{E}-02$ & $9.89 \mathrm{E}-04$ & $6.46 \mathrm{E}-05$ & $2.13 \mathrm{E}-06$ & $9.89 \mathrm{E}-12$ \\
\hline NB 93M & $6.34 \mathrm{E}-10$ & $6.38 \mathrm{E}-10$ & $6.41 \mathrm{E}-10$ & $6.70 \mathrm{E}-10$ & $7.43 \mathrm{E}-10$ & $9.58 \mathrm{E}-10$ & $1.10 \mathrm{E}-09$ & $1.28 \mathrm{E}-09$ & $1.91 \mathrm{E}-09$ \\
\hline
\end{tabular}


BSU-8242 3 DPA As-Run Physics Analysis

\begin{tabular}{|c|c|c|c|c|c|c|c|c|c|}
\hline \multicolumn{10}{|c|}{ ACTIVATION PRODUCTS } \\
\hline ISOTOPE & EOC & 1 Day & 2 Days & 10 Days & 30 Days & 90 Days & 130 Days & 180 Days & 360 Days \\
\hline NB 94 & $3.84 \mathrm{E}-03$ & $3.84 \mathrm{E}-03$ & $3.84 \mathrm{E}-03$ & $3.84 \mathrm{E}-03$ & $3.84 \mathrm{E}-03$ & $3.84 \mathrm{E}-03$ & $3.84 \mathrm{E}-03$ & $3.84 \mathrm{E}-03$ & $3.84 \mathrm{E}-03$ \\
\hline NB 95 & $9.48 \mathrm{E}+00$ & $9.30 \mathrm{E}+00$ & $9.12 \mathrm{E}+00$ & $7.79 \mathrm{E}+00$ & $5.25 \mathrm{E}+00$ & $1.61 \mathrm{E}+00$ & 7.33E-01 & $2.74 \mathrm{E}-01$ & 8.07E-03 \\
\hline NB 95M & $3.85 \mathrm{E}-05$ & $3.83 \mathrm{E}-05$ & $3.81 \mathrm{E}-05$ & $3.57 \mathrm{E}-05$ & $2.90 \mathrm{E}-05$ & $1.51 \mathrm{E}-05$ & $9.81 \mathrm{E}-06$ & $5.71 \mathrm{E}-06$ & $8.12 \mathrm{E}-07$ \\
\hline NB 96 & $5.01 \mathrm{E}-02$ & $2.46 \mathrm{E}-02$ & $1.21 \mathrm{E}-02$ & $4.03 \mathrm{E}-05$ & $2.62 \mathrm{E}-11$ & $7.12 \mathrm{E}-30$ & $0.00 \mathrm{E}+00$ & $0.00 \mathrm{E}+00$ & $0.00 \mathrm{E}+00$ \\
\hline NB 97 & $6.57 \mathrm{E}-03$ & $6.94 \mathrm{E}-05$ & $2.59 \mathrm{E}-05$ & $1.06 \mathrm{E}-08$ & $2.98 \mathrm{E}-17$ & $0.00 \mathrm{E}+00$ & $0.00 \mathrm{E}+00$ & $0.00 \mathrm{E}+00$ & $0.00 \mathrm{E}+00$ \\
\hline NB 97M & $1.75 \mathrm{E}-04$ & $6.54 \mathrm{E}-05$ & $2.44 \mathrm{E}-05$ & $9.29 \mathrm{E}-09$ & $2.62 \mathrm{E}-17$ & $0.00 \mathrm{E}+00$ & $0.00 \mathrm{E}+00$ & $0.00 \mathrm{E}+00$ & $0.00 \mathrm{E}+00$ \\
\hline NB 98 & $9.78 \mathrm{E}-04$ & $0.00 \mathrm{E}+00$ & $0.00 \mathrm{E}+00$ & $0.00 \mathrm{E}+00$ & $0.00 \mathrm{E}+00$ & $0.00 \mathrm{E}+00$ & $0.00 \mathrm{E}+00$ & $0.00 \mathrm{E}+00$ & $0.00 \mathrm{E}+00$ \\
\hline NB100 & $4.85 \mathrm{E}-05$ & $0.00 \mathrm{E}+00$ & $0.00 \mathrm{E}+00$ & $0.00 \mathrm{E}+00$ & $0.00 \mathrm{E}+00$ & $0.00 \mathrm{E}+00$ & $0.00 \mathrm{E}+00$ & $0.00 \mathrm{E}+00$ & $0.00 \mathrm{E}+00$ \\
\hline MO 93M & $1.35 \mathrm{E}-01$ & $1.19 \mathrm{E}-02$ & $1.05 \mathrm{E}-03$ & $3.84 \mathrm{E}-12$ & $0.00 \mathrm{E}+00$ & $0.00 \mathrm{E}+00$ & $0.00 \mathrm{E}+00$ & $0.00 \mathrm{E}+00$ & $0.00 \mathrm{E}+00$ \\
\hline MO 93 & 4.07E-04 & 4.07E-04 & 4.07E-04 & $4.07 \mathrm{E}-04$ & 4.07E-04 & 4.07E-04 & 4.07E-04 & 4.07E-04 & 4.07E-04 \\
\hline MO 99 & $9.46 \mathrm{E}+01$ & $7.35 \mathrm{E}+01$ & $5.72 \mathrm{E}+01$ & $7.61 \mathrm{E}+00$ & 4.92E-02 & $1.33 \mathrm{E}-08$ & $5.57 \mathrm{E}-13$ & $1.87 \mathrm{E}-18$ & $0.00 \mathrm{E}+00$ \\
\hline MO101 & $2.35 \mathrm{E}+01$ & $0.00 \mathrm{E}+00$ & $0.00 \mathrm{E}+00$ & $0.00 \mathrm{E}+00$ & $0.00 \mathrm{E}+00$ & $0.00 \mathrm{E}+00$ & $0.00 \mathrm{E}+00$ & $0.00 \mathrm{E}+00$ & $0.00 \mathrm{E}+00$ \\
\hline TC 99 & $1.73 \mathrm{E}-05$ & $1.74 \mathrm{E}-05$ & $1.75 \mathrm{E}-05$ & $1.77 \mathrm{E}-05$ & $1.77 \mathrm{E}-05$ & $1.77 \mathrm{E}-05$ & $1.77 \mathrm{E}-05$ & $1.77 \mathrm{E}-05$ & $1.77 \mathrm{E}-05$ \\
\hline TC100 & $1.44 \mathrm{E}+00$ & $0.00 \mathrm{E}+00$ & $0.00 \mathrm{E}+00$ & $0.00 \mathrm{E}+00$ & $0.00 \mathrm{E}+00$ & $0.00 \mathrm{E}+00$ & $0.00 \mathrm{E}+00$ & $0.00 \mathrm{E}+00$ & $0.00 \mathrm{E}+00$ \\
\hline $\mathrm{TC} 101$ & $2.35 \mathrm{E}+01$ & $0.00 \mathrm{E}+00$ & $0.00 \mathrm{E}+00$ & $0.00 \mathrm{E}+00$ & $0.00 \mathrm{E}+00$ & $0.00 \mathrm{E}+00$ & $0.00 \mathrm{E}+00$ & $0.00 \mathrm{E}+00$ & $0.00 \mathrm{E}+00$ \\
\hline RU103 & $1.01 \mathrm{E}-03$ & $9.93 \mathrm{E}-04$ & $9.76 \mathrm{E}-04$ & $8.47 \mathrm{E}-04$ & $5.95 \mathrm{E}-04$ & $2.07 \mathrm{E}-04$ & $1.02 \mathrm{E}-04$ & $4.22 \mathrm{E}-05$ & $1.76 \mathrm{E}-06$ \\
\hline RU105 & $1.20 \mathrm{E}-08$ & $2.82 \mathrm{E}-10$ & $6.65 \mathrm{E}-12$ & $6.34 \mathrm{E}-25$ & $0.00 \mathrm{E}+00$ & $0.00 \mathrm{E}+00$ & $0.00 \mathrm{E}+00$ & $0.00 \mathrm{E}+00$ & $0.00 \mathrm{E}+00$ \\
\hline RU106 & $4.89 \mathrm{E}-15$ & $4.88 \mathrm{E}-15$ & $4.87 \mathrm{E}-15$ & $4.80 \mathrm{E}-15$ & $4.62 \mathrm{E}-15$ & 4.13E-15 & $3.83 \mathrm{E}-15$ & $3.48 \mathrm{E}-15$ & $2.48 \mathrm{E}-15$ \\
\hline RH104 & $1.61 \mathrm{E}-05$ & $0.00 \mathrm{E}+00$ & $0.00 \mathrm{E}+00$ & $0.00 \mathrm{E}+00$ & $0.00 \mathrm{E}+00$ & $0.00 \mathrm{E}+00$ & $0.00 \mathrm{E}+00$ & $0.00 \mathrm{E}+00$ & $0.00 \mathrm{E}+00$ \\
\hline RH104M & $1.16 \mathrm{E}-06$ & $0.00 \mathrm{E}+00$ & $0.00 \mathrm{E}+00$ & $0.00 \mathrm{E}+00$ & $0.00 \mathrm{E}+00$ & $0.00 \mathrm{E}+00$ & $0.00 \mathrm{E}+00$ & $0.00 \mathrm{E}+00$ & $0.00 \mathrm{E}+00$ \\
\hline RH105 & $9.53 \mathrm{E}-09$ & $6.98 \mathrm{E}-09$ & 4.39E-09 & $1.02 \mathrm{E}-10$ & $8.35 \mathrm{E}-15$ & $4.60 \mathrm{E}-27$ & $0.00 \mathrm{E}+00$ & $0.00 \mathrm{E}+00$ & $0.00 \mathrm{E}+00$ \\
\hline RH105M & $3.35 \mathrm{E}-09$ & $7.93 \mathrm{E}-11$ & $1.87 \mathrm{E}-12$ & $1.78 \mathrm{E}-25$ & $0.00 \mathrm{E}+00$ & $0.00 \mathrm{E}+00$ & $0.00 \mathrm{E}+00$ & $0.00 \mathrm{E}+00$ & $0.00 \mathrm{E}+00$ \\
\hline RH106 & $1.67 \mathrm{E}-09$ & $4.88 \mathrm{E}-15$ & $4.87 \mathrm{E}-15$ & $4.80 \mathrm{E}-15$ & 4.62E-15 & 4.13E-15 & $3.83 \mathrm{E}-15$ & $3.48 \mathrm{E}-15$ & $2.48 \mathrm{E}-15$ \\
\hline RH106M & $8.12 \mathrm{E}-10$ & $4.22 \mathrm{E}-13$ & $2.20 \mathrm{E}-16$ & $0.00 \mathrm{E}+00$ & $0.00 \mathrm{E}+00$ & $0.00 \mathrm{E}+00$ & $0.00 \mathrm{E}+00$ & $0.00 \mathrm{E}+00$ & $0.00 \mathrm{E}+00$ \\
\hline PD107M & $6.01 \mathrm{E}-14$ & $0.00 \mathrm{E}+00$ & $0.00 \mathrm{E}+00$ & $0.00 \mathrm{E}+00$ & $0.00 \mathrm{E}+00$ & $0.00 \mathrm{E}+00$ & $0.00 \mathrm{E}+00$ & $0.00 \mathrm{E}+00$ & $0.00 \mathrm{E}+00$ \\
\hline PD109 & $2.48 \mathrm{E}-16$ & $7.68 \mathrm{E}-17$ & $1.64 \mathrm{E}-17$ & $8.33 \mathrm{E}-22$ & $2.28 \mathrm{E}-32$ & $0.00 \mathrm{E}+00$ & $0.00 \mathrm{E}+00$ & $0.00 \mathrm{E}+00$ & $0.00 \mathrm{E}+00$ \\
\hline
\end{tabular}


BSU-8242 3 DPA As-Run Physics Analysis

\begin{tabular}{|c|c|c|c|c|c|c|c|c|c|}
\hline \multicolumn{10}{|c|}{ ACTIVATION PRODUCTS } \\
\hline ISOTOPE & EOC & 1 Day & 2 Days & 10 Days & 30 Days & 90 Days & 130 Days & 180 Days & 360 Days \\
\hline AG109M & $2.47 \mathrm{E}-16$ & 7.69E-17 & $2.23 \mathrm{E}-17$ & $8.33 \mathrm{E}-22$ & $2.28 \mathrm{E}-32$ & $0.00 \mathrm{E}+00$ & $0.00 \mathrm{E}+00$ & $0.00 \mathrm{E}+00$ & $0.00 \mathrm{E}+00$ \\
\hline TA180 & $2.27 \mathrm{E}-14$ & $2.27 \mathrm{E}-14$ & $2.27 \mathrm{E}-14$ & $2.27 \mathrm{E}-14$ & $2.27 \mathrm{E}-14$ & $2.27 \mathrm{E}-14$ & $2.27 \mathrm{E}-14$ & $2.27 \mathrm{E}-14$ & $2.27 \mathrm{E}-14$ \\
\hline TA182 & $5.29 \mathrm{E}+02$ & $5.25 \mathrm{E}+02$ & $5.22 \mathrm{E}+02$ & $4.98 \mathrm{E}+02$ & $4.41 \mathrm{E}+02$ & $3.07 \mathrm{E}+02$ & $2.41 \mathrm{E}+02$ & $1.79 \mathrm{E}+02$ & $6.04 \mathrm{E}+01$ \\
\hline TA182M & $2.60 \mathrm{E}+00$ & $0.00 \mathrm{E}+00$ & $0.00 \mathrm{E}+00$ & $0.00 \mathrm{E}+00$ & $0.00 \mathrm{E}+00$ & $0.00 \mathrm{E}+00$ & $0.00 \mathrm{E}+00$ & $0.00 \mathrm{E}+00$ & $0.00 \mathrm{E}+00$ \\
\hline TA183 & $4.98 \mathrm{E}+03$ & $4.34 \mathrm{E}+03$ & $3.79 \mathrm{E}+03$ & $1.28 \mathrm{E}+03$ & $8.44 \mathrm{E}+01$ & $2.43 \mathrm{E}-02$ & $1.06 \mathrm{E}-04$ & $1.18 \mathrm{E}-07$ & $2.79 \mathrm{E}-18$ \\
\hline W181 & $3.01 \mathrm{E}-15$ & $3.00 \mathrm{E}-15$ & $2.98 \mathrm{E}-15$ & $2.85 \mathrm{E}-15$ & $2.54 \mathrm{E}-15$ & $1.80 \mathrm{E}-15$ & $1.43 \mathrm{E}-15$ & $1.08 \mathrm{E}-15$ & $3.84 \mathrm{E}-16$ \\
\hline W183M & $5.90 \mathrm{E}-01$ & $0.00 \mathrm{E}+00$ & $0.00 \mathrm{E}+00$ & $0.00 \mathrm{E}+00$ & $0.00 \mathrm{E}+00$ & $0.00 \mathrm{E}+00$ & $0.00 \mathrm{E}+00$ & $0.00 \mathrm{E}+00$ & $0.00 \mathrm{E}+00$ \\
\hline W185 & $7.91 \mathrm{E}-01$ & 7.84E-01 & $7.77 \mathrm{E}-01$ & 7.22E-01 & $6.00 \mathrm{E}-01$ & $3.45 \mathrm{E}-01$ & $2.38 \mathrm{E}-01$ & $1.50 \mathrm{E}-01$ & $2.85 \mathrm{E}-02$ \\
\hline $\mathrm{W} 185 \mathrm{M}$ & $2.48 \mathrm{E}-03$ & $0.00 \mathrm{E}+00$ & $0.00 \mathrm{E}+00$ & $0.00 \mathrm{E}+00$ & $0.00 \mathrm{E}+00$ & $0.00 \mathrm{E}+00$ & $0.00 \mathrm{E}+00$ & $0.00 \mathrm{E}+00$ & $0.00 \mathrm{E}+00$ \\
\hline W187 & $4.90 \mathrm{E}-04$ & $2.44 \mathrm{E}-04$ & $1.22 \mathrm{E}-04$ & $4.65 \mathrm{E}-07$ & $4.18 \mathrm{E}-13$ & $2.97 \mathrm{E}-31$ & $0.00 \mathrm{E}+00$ & $0.00 \mathrm{E}+00$ & $0.00 \mathrm{E}+00$ \\
\hline W188 & $1.10 \mathrm{E}-06$ & $1.09 \mathrm{E}-06$ & $1.08 \mathrm{E}-06$ & $9.96 \mathrm{E}-07$ & $8.16 \mathrm{E}-07$ & $4.48 \mathrm{E}-07$ & $3.00 \mathrm{E}-07$ & $1.82 \mathrm{E}-07$ & $3.02 \mathrm{E}-08$ \\
\hline RE186 & $1.52 \mathrm{E}-01$ & $1.26 \mathrm{E}-01$ & $1.05 \mathrm{E}-01$ & $2.42 \mathrm{E}-02$ & $6.16 \mathrm{E}-04$ & $1.02 \mathrm{E}-08$ & $6.59 \mathrm{E}-12$ & $6.82 \mathrm{E}-16$ & $3.06 \mathrm{E}-30$ \\
\hline RE187 & $6.34 \mathrm{E}-16$ & $6.47 \mathrm{E}-16$ & $6.54 \mathrm{E}-16$ & $6.60 \mathrm{E}-16$ & $6.60 \mathrm{E}-16$ & $6.60 \mathrm{E}-16$ & $6.60 \mathrm{E}-16$ & $6.60 \mathrm{E}-16$ & $6.60 \mathrm{E}-16$ \\
\hline RE188 & $1.92 \mathrm{E}-05$ & $8.02 \mathrm{E}-06$ & $3.69 \mathrm{E}-06$ & $1.01 \mathrm{E}-06$ & $8.24 \mathrm{E}-07$ & $4.53 \mathrm{E}-07$ & $3.04 \mathrm{E}-07$ & $1.84 \mathrm{E}-07$ & $3.05 \mathrm{E}-08$ \\
\hline RE188M & $1.77 \mathrm{E}-05$ & $0.00 \mathrm{E}+00$ & $0.00 \mathrm{E}+00$ & $0.00 \mathrm{E}+00$ & $0.00 \mathrm{E}+00$ & $0.00 \mathrm{E}+00$ & $0.00 \mathrm{E}+00$ & $0.00 \mathrm{E}+00$ & $0.00 \mathrm{E}+00$ \\
\hline RE189 & $2.92 \mathrm{E}-10$ & $1.48 \mathrm{E}-10$ & $7.44 \mathrm{E}-11$ & $3.11 \mathrm{E}-13$ & $3.52 \mathrm{E}-19$ & $0.00 \mathrm{E}+00$ & $0.00 \mathrm{E}+00$ & $0.00 \mathrm{E}+00$ & $0.00 \mathrm{E}+00$ \\
\hline OS190M & $7.20 \mathrm{E}-14$ & $0.00 \mathrm{E}+00$ & $0.00 \mathrm{E}+00$ & $0.00 \mathrm{E}+00$ & $0.00 \mathrm{E}+00$ & $0.00 \mathrm{E}+00$ & $0.00 \mathrm{E}+00$ & $0.00 \mathrm{E}+00$ & $0.00 \mathrm{E}+00$ \\
\hline OS191 & $9.59 \mathrm{E}-12$ & $9.52 \mathrm{E}-12$ & $9.20 \mathrm{E}-12$ & $6.45 \mathrm{E}-12$ & $2.62 \mathrm{E}-12$ & $1.76 \mathrm{E}-13$ & $2.91 \mathrm{E}-14$ & $3.07 \mathrm{E}-15$ & $9.33 \mathrm{E}-19$ \\
\hline OS191M & $1.42 \mathrm{E}-11$ & $3.96 \mathrm{E}-12$ & $1.10 \mathrm{E}-12$ & $3.94 \mathrm{E}-17$ & $3.02 \mathrm{E}-28$ & $0.00 \mathrm{E}+00$ & $0.00 \mathrm{E}+00$ & $0.00 \mathrm{E}+00$ & $0.00 \mathrm{E}+00$ \\
\hline IR192 & $2.31 \mathrm{E}-13$ & $2.29 \mathrm{E}-13$ & $2.26 \mathrm{E}-13$ & $2.10 \mathrm{E}-13$ & $1.74 \mathrm{E}-13$ & $9.93 \mathrm{E}-14$ & $6.83 \mathrm{E}-14$ & $4.27 \mathrm{E}-14$ & $7.92 \mathrm{E}-15$ \\
\hline IR194 & $9.80 \mathrm{E}-15$ & $4.11 \mathrm{E}-15$ & $1.72 \mathrm{E}-15$ & $1.65 \mathrm{E}-18$ & $4.70 \mathrm{E}-26$ & $0.00 \mathrm{E}+00$ & $0.00 \mathrm{E}+00$ & $0.00 \mathrm{E}+00$ & $0.00 \mathrm{E}+00$ \\
\hline IR194M & $5.17 \mathrm{E}-16$ & $0.00 \mathrm{E}+00$ & $0.00 \mathrm{E}+00$ & $0.00 \mathrm{E}+00$ & $0.00 \mathrm{E}+00$ & $0.00 \mathrm{E}+00$ & $0.00 \mathrm{E}+00$ & $0.00 \mathrm{E}+00$ & $0.00 \mathrm{E}+00$ \\
\hline PT193M & $1.39 \mathrm{E}-16$ & $1.17 \mathrm{E}-16$ & $9.79 \mathrm{E}-17$ & $2.94 \mathrm{E}-17$ & 4.13E-19 & $2.60 \mathrm{E}-23$ & $2.60 \mathrm{E}-23$ & $8.22 \mathrm{E}-27$ & $0.00 \mathrm{E}+00$ \\
\hline SUMTOT & $1.24 \mathrm{E}+04$ & $7.75 \mathrm{E}+03$ & $7.09 \mathrm{E}+03$ & $4.08 \mathrm{E}+03$ & $2.08 \mathrm{E}+03$ & $9.24 \mathrm{E}+02$ & $6.66 \mathrm{E}+02$ & $5.06 \mathrm{E}+02$ & $3.02 \mathrm{E}+02$ \\
\hline 0TOTAL & $1.24 \mathrm{E}+04$ & $7.75 \mathrm{E}+03$ & $7.09 \mathrm{E}+03$ & $4.08 \mathrm{E}+03$ & $2.08 \mathrm{E}+03$ & $9.24 \mathrm{E}+02$ & $6.66 \mathrm{E}+02$ & $5.06 \mathrm{E}+02$ & $3.02 \mathrm{E}+02$ \\
\hline
\end{tabular}


Table 22. Source Terms for 'A6 300C 3 DPA' Capsule.

\begin{tabular}{|c|c|c|c|c|c|c|c|c|c|}
\hline \multicolumn{10}{|c|}{ ACTIVATION PRODUCTS (grams) } \\
\hline ISOTOPE & EOC & 1 Day & 2 Days & 10 Days & 30 Days & 90 Days & 130 Days & 180 Days & 360 Days \\
\hline H 1 & $1.12 \mathrm{E}-03$ & $1.12 \mathrm{E}-03$ & $1.12 \mathrm{E}-03$ & $1.12 \mathrm{E}-03$ & $1.12 \mathrm{E}-03$ & $1.12 \mathrm{E}-03$ & $1.12 \mathrm{E}-03$ & $1.12 \mathrm{E}-03$ & $1.12 \mathrm{E}-03$ \\
\hline $\mathrm{H} 2$ & $6.17 \mathrm{E}-07$ & $6.17 \mathrm{E}-07$ & $6.17 \mathrm{E}-07$ & $6.17 \mathrm{E}-07$ & $6.17 \mathrm{E}-07$ & $6.17 \mathrm{E}-07$ & $6.17 \mathrm{E}-07$ & $6.17 \mathrm{E}-07$ & $6.17 \mathrm{E}-07$ \\
\hline H 3 & 9.03E-09 & $9.03 \mathrm{E}-09$ & $9.03 \mathrm{E}-09$ & $9.02 \mathrm{E}-09$ & 8.99E-09 & 8.91E-09 & $8.86 \mathrm{E}-09$ & 8.79E-09 & $8.55 \mathrm{E}-09$ \\
\hline HE 3 & 3.32E-11 & $3.46 \mathrm{E}-11$ & $3.60 \mathrm{E}-11$ & $4.71 \mathrm{E}-11$ & $7.47 \mathrm{E}-11$ & $1.57 \mathrm{E}-10$ & $2.12 \mathrm{E}-10$ & $2.80 \mathrm{E}-10$ & $5.19 \mathrm{E}-10$ \\
\hline HE 4 & $1.14 \mathrm{E}-02$ & $1.14 \mathrm{E}-02$ & $1.14 \mathrm{E}-02$ & $1.14 \mathrm{E}-02$ & $1.14 \mathrm{E}-02$ & $1.14 \mathrm{E}-02$ & $1.14 \mathrm{E}-02$ & $1.14 \mathrm{E}-02$ & $1.14 \mathrm{E}-02$ \\
\hline HE 6 & $8.85 \mathrm{E}-17$ & $0.00 \mathrm{E}+00$ & $0.00 \mathrm{E}+00$ & $0.00 \mathrm{E}+00$ & $0.00 \mathrm{E}+00$ & $0.00 \mathrm{E}+00$ & $0.00 \mathrm{E}+00$ & $0.00 \mathrm{E}+00$ & $0.00 \mathrm{E}+00$ \\
\hline LI 6 & $2.55 \mathrm{E}-10$ & $2.55 \mathrm{E}-10$ & $2.55 \mathrm{E}-10$ & $2.55 \mathrm{E}-10$ & $2.55 \mathrm{E}-10$ & $2.55 \mathrm{E}-10$ & $2.55 \mathrm{E}-10$ & $2.55 \mathrm{E}-10$ & $2.55 \mathrm{E}-10$ \\
\hline LI 7 & $1.40 \mathrm{E}-14$ & $1.40 \mathrm{E}-14$ & $1.40 \mathrm{E}-14$ & $1.40 \mathrm{E}-14$ & $1.40 \mathrm{E}-14$ & $1.40 \mathrm{E}-14$ & $1.40 \mathrm{E}-14$ & $1.40 \mathrm{E}-14$ & $1.40 \mathrm{E}-14$ \\
\hline BE 9 & $2.92 \mathrm{E}-06$ & $2.92 \mathrm{E}-06$ & $2.92 \mathrm{E}-06$ & $2.92 \mathrm{E}-06$ & $2.92 \mathrm{E}-06$ & $2.92 \mathrm{E}-06$ & $2.92 \mathrm{E}-06$ & $2.92 \mathrm{E}-06$ & $2.92 \mathrm{E}-06$ \\
\hline BE 10 & 7.47E-08 & 7.47E-08 & 7.47E-08 & 7.47E-08 & 7.47E-08 & 7.47E-08 & $7.47 \mathrm{E}-08$ & $7.47 \mathrm{E}-08$ & 7.47E-08 \\
\hline B 10 & $2.41 \mathrm{E}-15$ & $2.50 \mathrm{E}-15$ & $2.59 \mathrm{E}-15$ & $3.29 \mathrm{E}-15$ & $5.07 \mathrm{E}-15$ & $1.04 \mathrm{E}-14$ & $1.39 \mathrm{E}-14$ & $1.84 \mathrm{E}-14$ & $3.43 \mathrm{E}-14$ \\
\hline B 11 & $6.01 \mathrm{E}-14$ & $6.01 \mathrm{E}-14$ & $6.01 \mathrm{E}-14$ & $6.01 \mathrm{E}-14$ & $6.01 \mathrm{E}-14$ & $6.01 \mathrm{E}-14$ & $6.01 \mathrm{E}-14$ & $6.01 \mathrm{E}-14$ & $6.01 \mathrm{E}-14$ \\
\hline C 12 & $6.30 \mathrm{E}-01$ & $6.30 \mathrm{E}-01$ & $6.30 \mathrm{E}-01$ & $6.30 \mathrm{E}-01$ & $6.30 \mathrm{E}-01$ & $6.30 \mathrm{E}-01$ & $6.30 \mathrm{E}-01$ & $6.30 \mathrm{E}-01$ & $6.30 \mathrm{E}-01$ \\
\hline C 13 & 7.67E-03 & 7.67E-03 & 7.67E-03 & 7.67E-03 & 7.67E-03 & 7.67E-03 & $7.67 \mathrm{E}-03$ & $7.67 \mathrm{E}-03$ & 7.67E-03 \\
\hline C 14 & $1.37 \mathrm{E}-08$ & $1.37 \mathrm{E}-08$ & $1.37 \mathrm{E}-08$ & $1.37 \mathrm{E}-08$ & $1.37 \mathrm{E}-08$ & $1.37 \mathrm{E}-08$ & $1.37 \mathrm{E}-08$ & $1.37 \mathrm{E}-08$ & $1.37 \mathrm{E}-08$ \\
\hline N 14 & $7.90 \mathrm{E}-13$ & $7.95 \mathrm{E}-13$ & $8.00 \mathrm{E}-13$ & $8.36 \mathrm{E}-13$ & $9.27 \mathrm{E}-13$ & $1.20 \mathrm{E}-12$ & $1.38 \mathrm{E}-12$ & $1.61 \mathrm{E}-12$ & $2.43 \mathrm{E}-12$ \\
\hline N 15 & $7.75 \mathrm{E}-15$ & $7.75 \mathrm{E}-15$ & $7.75 \mathrm{E}-15$ & $7.75 \mathrm{E}-15$ & $7.75 \mathrm{E}-15$ & $7.75 \mathrm{E}-15$ & $7.75 \mathrm{E}-15$ & $7.75 \mathrm{E}-15$ & $7.75 \mathrm{E}-15$ \\
\hline O 17 & $4.05 \mathrm{E}-17$ & $4.05 \mathrm{E}-17$ & $4.05 \mathrm{E}-17$ & $4.05 \mathrm{E}-17$ & 4.05E-17 & $4.05 \mathrm{E}-17$ & $4.05 \mathrm{E}-17$ & $4.05 \mathrm{E}-17$ & $4.05 \mathrm{E}-17$ \\
\hline O 18 & $3.80 \mathrm{E}-09$ & $3.80 \mathrm{E}-09$ & $3.80 \mathrm{E}-09$ & $3.80 \mathrm{E}-09$ & $3.80 \mathrm{E}-09$ & $3.80 \mathrm{E}-09$ & $3.80 \mathrm{E}-09$ & $3.80 \mathrm{E}-09$ & $3.80 \mathrm{E}-09$ \\
\hline F 19 & $6.97 \mathrm{E}-16$ & $6.97 \mathrm{E}-16$ & $6.97 \mathrm{E}-16$ & $6.97 \mathrm{E}-16$ & $6.97 \mathrm{E}-16$ & $6.97 \mathrm{E}-16$ & $6.97 \mathrm{E}-16$ & $6.97 \mathrm{E}-16$ & $6.97 \mathrm{E}-16$ \\
\hline NE 20 & $1.47 \mathrm{E}-12$ & $1.47 \mathrm{E}-12$ & $1.47 \mathrm{E}-12$ & $1.47 \mathrm{E}-12$ & $1.47 \mathrm{E}-12$ & $1.47 \mathrm{E}-12$ & $1.47 \mathrm{E}-12$ & $1.47 \mathrm{E}-12$ & $1.47 \mathrm{E}-12$ \\
\hline
\end{tabular}


BSU-8242 3 DPA As-Run Physics Analysis

\begin{tabular}{|c|c|c|c|c|c|c|c|c|c|}
\hline \multicolumn{10}{|c|}{ ACTIVATION PRODUCTS (grams) } \\
\hline ISOTOPE & EOC & 1 Day & 2 Days & 10 Days & 30 Days & 90 Days & 130 Days & 180 Days & 360 Days \\
\hline NE 21 & 4.04E-06 & 4.04E-06 & $4.04 \mathrm{E}-06$ & $4.04 \mathrm{E}-06$ & $4.04 \mathrm{E}-06$ & $4.04 \mathrm{E}-06$ & 4.04E-06 & $4.04 \mathrm{E}-06$ & 4.04E-06 \\
\hline NE 22 & $6.07 \mathrm{E}-06$ & $6.07 \mathrm{E}-06$ & $6.07 \mathrm{E}-06$ & $6.07 \mathrm{E}-06$ & $6.07 \mathrm{E}-06$ & $6.07 \mathrm{E}-06$ & $6.07 \mathrm{E}-06$ & $6.07 \mathrm{E}-06$ & $6.07 \mathrm{E}-06$ \\
\hline NE 23 & $4.26 \mathrm{E}-12$ & $0.00 \mathrm{E}+00$ & $0.00 \mathrm{E}+00$ & $0.00 \mathrm{E}+00$ & $0.00 \mathrm{E}+00$ & $0.00 \mathrm{E}+00$ & $0.00 \mathrm{E}+00$ & $0.00 \mathrm{E}+00$ & $0.00 \mathrm{E}+00$ \\
\hline NA 23 & $1.23 \mathrm{E}-06$ & $1.23 \mathrm{E}-06$ & $1.23 \mathrm{E}-06$ & $1.23 \mathrm{E}-06$ & $1.23 \mathrm{E}-06$ & $1.23 \mathrm{E}-06$ & $1.23 \mathrm{E}-06$ & $1.23 \mathrm{E}-06$ & $1.23 \mathrm{E}-06$ \\
\hline NA 24 & $1.59 \mathrm{E}-06$ & $5.24 \mathrm{E}-07$ & $1.73 \mathrm{E}-07$ & $2.42 \mathrm{E}-11$ & 5.64E-21 & $0.00 \mathrm{E}+00$ & $0.00 \mathrm{E}+00$ & $0.00 \mathrm{E}+00$ & $0.00 \mathrm{E}+00$ \\
\hline NA 25 & $4.47 \mathrm{E}-12$ & $0.00 \mathrm{E}+00$ & $0.00 \mathrm{E}+00$ & $0.00 \mathrm{E}+00$ & $0.00 \mathrm{E}+00$ & $0.00 \mathrm{E}+00$ & $0.00 \mathrm{E}+00$ & $0.00 \mathrm{E}+00$ & $0.00 \mathrm{E}+00$ \\
\hline MG 24 & $6.32 \mathrm{E}-01$ & $6.32 \mathrm{E}-01$ & $6.32 \mathrm{E}-01$ & $6.32 \mathrm{E}-01$ & $6.32 \mathrm{E}-01$ & $6.32 \mathrm{E}-01$ & $6.32 \mathrm{E}-01$ & $6.32 \mathrm{E}-01$ & $6.32 \mathrm{E}-01$ \\
\hline MG 25 & $8.34 \mathrm{E}-02$ & $8.34 \mathrm{E}-02$ & $8.34 \mathrm{E}-02$ & $8.34 \mathrm{E}-02$ & $8.34 \mathrm{E}-02$ & $8.34 \mathrm{E}-02$ & $8.34 \mathrm{E}-02$ & $8.34 \mathrm{E}-02$ & $8.34 \mathrm{E}-02$ \\
\hline MG 26 & $9.55 \mathrm{E}-02$ & $9.55 \mathrm{E}-02$ & $9.55 \mathrm{E}-02$ & $9.55 \mathrm{E}-02$ & $9.55 \mathrm{E}-02$ & $9.55 \mathrm{E}-02$ & $9.55 \mathrm{E}-02$ & $9.55 \mathrm{E}-02$ & $9.55 \mathrm{E}-02$ \\
\hline MG 27 & $8.89 \mathrm{E}-08$ & $0.00 \mathrm{E}+00$ & $0.00 \mathrm{E}+00$ & $0.00 \mathrm{E}+00$ & $0.00 \mathrm{E}+00$ & $0.00 \mathrm{E}+00$ & $0.00 \mathrm{E}+00$ & $0.00 \mathrm{E}+00$ & $0.00 \mathrm{E}+00$ \\
\hline MG 28 & $3.76 \mathrm{E}-14$ & $1.70 \mathrm{E}-14$ & $7.65 \mathrm{E}-15$ & $1.32 \mathrm{E}-17$ & $1.62 \mathrm{E}-24$ & $0.00 \mathrm{E}+00$ & $0.00 \mathrm{E}+00$ & $0.00 \mathrm{E}+00$ & $0.00 \mathrm{E}+00$ \\
\hline AL 27 & $1.12 \mathrm{E}+02$ & $1.12 \mathrm{E}+02$ & $1.12 \mathrm{E}+02$ & $1.12 \mathrm{E}+02$ & $1.12 \mathrm{E}+02$ & $1.12 \mathrm{E}+02$ & $1.12 \mathrm{E}+02$ & $1.12 \mathrm{E}+02$ & $1.12 \mathrm{E}+02$ \\
\hline AL 28 & $5.22 \mathrm{E}-07$ & $3.03 \mathrm{E}-17$ & $1.37 \mathrm{E}-17$ & $2.36 \mathrm{E}-20$ & $2.90 \mathrm{E}-27$ & $0.00 \mathrm{E}+00$ & $0.00 \mathrm{E}+00$ & $0.00 \mathrm{E}+00$ & $0.00 \mathrm{E}+00$ \\
\hline AL 29 & $3.24 \mathrm{E}-11$ & $0.00 \mathrm{E}+00$ & $0.00 \mathrm{E}+00$ & $0.00 \mathrm{E}+00$ & $0.00 \mathrm{E}+00$ & $0.00 \mathrm{E}+00$ & $0.00 \mathrm{E}+00$ & $0.00 \mathrm{E}+00$ & $0.00 \mathrm{E}+00$ \\
\hline AL 30 & $3.05 \mathrm{E}-16$ & $0.00 \mathrm{E}+00$ & $0.00 \mathrm{E}+00$ & $0.00 \mathrm{E}+00$ & $0.00 \mathrm{E}+00$ & $0.00 \mathrm{E}+00$ & $0.00 \mathrm{E}+00$ & $0.00 \mathrm{E}+00$ & $0.00 \mathrm{E}+00$ \\
\hline SI 28 & $1.33 \mathrm{E}+00$ & $1.33 \mathrm{E}+00$ & $1.33 \mathrm{E}+00$ & $1.33 \mathrm{E}+00$ & $1.33 \mathrm{E}+00$ & $1.33 \mathrm{E}+00$ & $1.33 \mathrm{E}+00$ & $1.33 \mathrm{E}+00$ & $1.33 \mathrm{E}+00$ \\
\hline SI 29 & $6.80 \mathrm{E}-02$ & $6.80 \mathrm{E}-02$ & $6.80 \mathrm{E}-02$ & $6.80 \mathrm{E}-02$ & $6.80 \mathrm{E}-02$ & $6.80 \mathrm{E}-02$ & $6.80 \mathrm{E}-02$ & $6.80 \mathrm{E}-02$ & $6.80 \mathrm{E}-02$ \\
\hline SI 30 & 4.65E-02 & $4.65 \mathrm{E}-02$ & $4.65 \mathrm{E}-02$ & $4.65 \mathrm{E}-02$ & $4.65 \mathrm{E}-02$ & $4.65 \mathrm{E}-02$ & $4.65 \mathrm{E}-02$ & $4.65 \mathrm{E}-02$ & $4.65 \mathrm{E}-02$ \\
\hline SI 31 & $8.78 \mathrm{E}-09$ & $1.54 \mathrm{E}-11$ & $2.69 \mathrm{E}-14$ & $0.00 \mathrm{E}+00$ & $0.00 \mathrm{E}+00$ & $0.00 \mathrm{E}+00$ & $0.00 \mathrm{E}+00$ & $0.00 \mathrm{E}+00$ & $0.00 \mathrm{E}+00$ \\
\hline SI 32 & $6.10 \mathrm{E}-12$ & $6.10 \mathrm{E}-12$ & $6.10 \mathrm{E}-12$ & $6.10 \mathrm{E}-12$ & $6.10 \mathrm{E}-12$ & $6.09 \mathrm{E}-12$ & $6.09 \mathrm{E}-12$ & $6.09 \mathrm{E}-12$ & $6.09 \mathrm{E}-12$ \\
\hline P 31 & $9.49 \mathrm{E}-03$ & $9.49 \mathrm{E}-03$ & $9.49 \mathrm{E}-03$ & $9.49 \mathrm{E}-03$ & $9.49 \mathrm{E}-03$ & $9.49 \mathrm{E}-03$ & $9.49 \mathrm{E}-03$ & $9.49 \mathrm{E}-03$ & $9.49 \mathrm{E}-03$ \\
\hline P 32 & $8.45 \mathrm{E}-07$ & $8.05 \mathrm{E}-07$ & $7.67 \mathrm{E}-07$ & $5.21 \mathrm{E}-07$ & $1.98 \mathrm{E}-07$ & $1.08 \mathrm{E}-08$ & $1.55 \mathrm{E}-09$ & $1.37 \mathrm{E}-10$ & $2.27 \mathrm{E}-14$ \\
\hline P 33 & $7.94 \mathrm{E}-11$ & $7.72 \mathrm{E}-11$ & $7.51 \mathrm{E}-11$ & $6.02 \mathrm{E}-11$ & $3.46 \mathrm{E}-11$ & $6.55 \mathrm{E}-12$ & $2.16 \mathrm{E}-12$ & $5.40 \mathrm{E}-13$ & $3.67 \mathrm{E}-15$ \\
\hline P 34 & $2.92 \mathrm{E}-15$ & $0.00 \mathrm{E}+00$ & $0.00 \mathrm{E}+00$ & $0.00 \mathrm{E}+00$ & $0.00 \mathrm{E}+00$ & $0.00 \mathrm{E}+00$ & $0.00 \mathrm{E}+00$ & $0.00 \mathrm{E}+00$ & $0.00 \mathrm{E}+00$ \\
\hline S 32 & $1.76 \mathrm{E}-02$ & $1.76 \mathrm{E}-02$ & $1.76 \mathrm{E}-02$ & $1.76 \mathrm{E}-02$ & $1.76 \mathrm{E}-02$ & $1.76 \mathrm{E}-02$ & $1.76 \mathrm{E}-02$ & $1.76 \mathrm{E}-02$ & $1.76 \mathrm{E}-02$ \\
\hline S 33 & $1.57 \mathrm{E}-04$ & $1.57 \mathrm{E}-04$ & $1.57 \mathrm{E}-04$ & $1.57 \mathrm{E}-04$ & $1.57 \mathrm{E}-04$ & $1.57 \mathrm{E}-04$ & $1.57 \mathrm{E}-04$ & $1.57 \mathrm{E}-04$ & $1.57 \mathrm{E}-04$ \\
\hline
\end{tabular}


BSU-8242 3 DPA As-Run Physics Analysis

\begin{tabular}{|c|c|c|c|c|c|c|c|c|c|}
\hline \multicolumn{10}{|c|}{ ACTIVATION PRODUCTS (grams) } \\
\hline ISOTOPE & EOC & 1 Day & 2 Days & 10 Days & 30 Days & 90 Days & 130 Days & 180 Days & 360 Days \\
\hline S 34 & $8.29 \mathrm{E}-04$ & $8.29 \mathrm{E}-04$ & $8.29 \mathrm{E}-04$ & $8.29 \mathrm{E}-04$ & $8.29 \mathrm{E}-04$ & $8.29 \mathrm{E}-04$ & $8.29 \mathrm{E}-04$ & $8.29 \mathrm{E}-04$ & $8.29 \mathrm{E}-04$ \\
\hline S 35 & $1.24 \mathrm{E}-07$ & $1.23 \mathrm{E}-07$ & $1.22 \mathrm{E}-07$ & $1.15 \mathrm{E}-07$ & $9.78 \mathrm{E}-08$ & $6.10 \mathrm{E}-08$ & $4.45 \mathrm{E}-08$ & $3.00 \mathrm{E}-08$ & $7.27 \mathrm{E}-09$ \\
\hline S 36 & $3.54 \mathrm{E}-06$ & $3.54 \mathrm{E}-06$ & $3.54 \mathrm{E}-06$ & $3.54 \mathrm{E}-06$ & $3.54 \mathrm{E}-06$ & $3.54 \mathrm{E}-06$ & $3.54 \mathrm{E}-06$ & $3.54 \mathrm{E}-06$ & $3.54 \mathrm{E}-06$ \\
\hline S 37 & $2.25 \mathrm{E}-14$ & $0.00 \mathrm{E}+00$ & $0.00 \mathrm{E}+00$ & $0.00 \mathrm{E}+00$ & $0.00 \mathrm{E}+00$ & $0.00 \mathrm{E}+00$ & $0.00 \mathrm{E}+00$ & $0.00 \mathrm{E}+00$ & $0.00 \mathrm{E}+00$ \\
\hline CL 35 & $1.70 \mathrm{E}-07$ & $1.71 \mathrm{E}-07$ & $1.72 \mathrm{E}-07$ & $1.80 \mathrm{E}-07$ & $1.96 \mathrm{E}-07$ & $2.33 \mathrm{E}-07$ & $2.50 \mathrm{E}-07$ & $2.64 \mathrm{E}-07$ & $2.87 \mathrm{E}-07$ \\
\hline CL 36 & $5.20 \mathrm{E}-09$ & $5.20 \mathrm{E}-09$ & $5.20 \mathrm{E}-09$ & $5.20 \mathrm{E}-09$ & $5.20 \mathrm{E}-09$ & $5.20 \mathrm{E}-09$ & $5.20 \mathrm{E}-09$ & $5.20 \mathrm{E}-09$ & $5.20 \mathrm{E}-09$ \\
\hline CL 37 & $8.22 \mathrm{E}-10$ & $8.22 \mathrm{E}-10$ & $8.22 \mathrm{E}-10$ & $8.22 \mathrm{E}-10$ & $8.22 \mathrm{E}-10$ & $8.22 \mathrm{E}-10$ & $8.22 \mathrm{E}-10$ & $8.22 \mathrm{E}-10$ & $8.22 \mathrm{E}-10$ \\
\hline CL 38 & $1.17 \mathrm{E}-16$ & $2.64 \mathrm{E}-28$ & $5.94 \mathrm{E}-40$ & $0.00 \mathrm{E}+00$ & $0.00 \mathrm{E}+00$ & $0.00 \mathrm{E}+00$ & $0.00 \mathrm{E}+00$ & $0.00 \mathrm{E}+00$ & $0.00 \mathrm{E}+00$ \\
\hline AR 36 & $2.36 \mathrm{E}-15$ & $2.39 \mathrm{E}-15$ & $2.43 \mathrm{E}-15$ & $2.68 \mathrm{E}-15$ & $3.33 \mathrm{E}-15$ & $5.26 \mathrm{E}-15$ & $6.54 \mathrm{E}-15$ & $8.15 \mathrm{E}-15$ & $1.39 \mathrm{E}-14$ \\
\hline AR 38 & $2.79 \mathrm{E}-13$ & $2.79 \mathrm{E}-13$ & $2.79 \mathrm{E}-13$ & $2.79 \mathrm{E}-13$ & $2.79 \mathrm{E}-13$ & $2.79 \mathrm{E}-13$ & $2.79 \mathrm{E}-13$ & $2.79 \mathrm{E}-13$ & $2.79 \mathrm{E}-13$ \\
\hline AR 39 & $9.14 \mathrm{E}-17$ & $9.14 \mathrm{E}-17$ & $9.14 \mathrm{E}-17$ & $9.14 \mathrm{E}-17$ & $9.14 \mathrm{E}-17$ & $9.14 \mathrm{E}-17$ & $9.13 \mathrm{E}-17$ & $9.13 \mathrm{E}-17$ & $9.12 \mathrm{E}-17$ \\
\hline AR 40 & $1.83 \mathrm{E}-12$ & $1.83 \mathrm{E}-12$ & $1.83 \mathrm{E}-12$ & $1.83 \mathrm{E}-12$ & $1.83 \mathrm{E}-12$ & $1.83 \mathrm{E}-12$ & $1.83 \mathrm{E}-12$ & $1.83 \mathrm{E}-12$ & $1.83 \mathrm{E}-12$ \\
\hline AR 41 & $7.27 \mathrm{E}-17$ & $8.08 \mathrm{E}-21$ & $8.97 \mathrm{E}-25$ & $0.00 \mathrm{E}+00$ & $0.00 \mathrm{E}+00$ & $0.00 \mathrm{E}+00$ & $0.00 \mathrm{E}+00$ & $0.00 \mathrm{E}+00$ & $0.00 \mathrm{E}+00$ \\
\hline K 41 & $5.93 \mathrm{E}-14$ & $5.94 \mathrm{E}-14$ & $5.94 \mathrm{E}-14$ & $5.94 \mathrm{E}-14$ & $5.94 \mathrm{E}-14$ & $5.94 \mathrm{E}-14$ & $5.94 \mathrm{E}-14$ & $5.94 \mathrm{E}-14$ & $5.94 \mathrm{E}-14$ \\
\hline K 43 & $1.20 \mathrm{E}-14$ & $5.72 \mathrm{E}-15$ & $2.74 \mathrm{E}-15$ & $7.60 \mathrm{E}-18$ & $3.07 \mathrm{E}-24$ & $1.81 \mathrm{E}-43$ & $0.00 \mathrm{E}+00$ & $0.00 \mathrm{E}+00$ & $0.00 \mathrm{E}+00$ \\
\hline K 44 & $3.52 \mathrm{E}-17$ & $6.97 \mathrm{E}-37$ & $0.00 \mathrm{E}+00$ & $0.00 \mathrm{E}+00$ & $0.00 \mathrm{E}+00$ & $0.00 \mathrm{E}+00$ & $0.00 \mathrm{E}+00$ & $0.00 \mathrm{E}+00$ & $0.00 \mathrm{E}+00$ \\
\hline CA 42 & $5.74 \mathrm{E}-16$ & $5.78 \mathrm{E}-16$ & $5.79 \mathrm{E}-16$ & $5.79 \mathrm{E}-16$ & $5.79 \mathrm{E}-16$ & $5.79 \mathrm{E}-16$ & $5.79 \mathrm{E}-16$ & $5.79 \mathrm{E}-16$ & $5.79 \mathrm{E}-16$ \\
\hline CA 43 & $9.59 \mathrm{E}-08$ & $9.59 \mathrm{E}-08$ & $9.59 \mathrm{E}-08$ & $9.59 \mathrm{E}-08$ & $9.59 \mathrm{E}-08$ & $9.59 \mathrm{E}-08$ & $9.59 \mathrm{E}-08$ & $9.59 \mathrm{E}-08$ & $9.59 \mathrm{E}-08$ \\
\hline CA 44 & $7.40 \mathrm{E}-07$ & $7.40 \mathrm{E}-07$ & $7.40 \mathrm{E}-07$ & $7.40 \mathrm{E}-07$ & $7.40 \mathrm{E}-07$ & $7.40 \mathrm{E}-07$ & $7.40 \mathrm{E}-07$ & $7.40 \mathrm{E}-07$ & $7.40 \mathrm{E}-07$ \\
\hline CA 45 & 8.84E-09 & $8.80 \mathrm{E}-09$ & $8.76 \mathrm{E}-09$ & $8.47 \mathrm{E}-09$ & 7.78E-09 & $6.03 \mathrm{E}-09$ & $5.08 \mathrm{E}-09$ & 4.11E-09 & $1.91 \mathrm{E}-09$ \\
\hline CA 46 & 8.88E-08 & 8.88E-08 & $8.88 \mathrm{E}-08$ & 8.88E-08 & 8.88E-08 & $8.88 \mathrm{E}-08$ & 8.88E-08 & 8.88E-08 & $8.88 \mathrm{E}-08$ \\
\hline CA 47 & $4.86 \mathrm{E}-12$ & $4.17 \mathrm{E}-12$ & $3.58 \mathrm{E}-12$ & $1.06 \mathrm{E}-12$ & 4.97E-14 & $5.18 \mathrm{E}-18$ & $1.15 \mathrm{E}-20$ & $5.51 \mathrm{E}-24$ & $6.24 \mathrm{E}-36$ \\
\hline $\mathrm{SC} 45$ & $6.59 \mathrm{E}-09$ & $6.63 \mathrm{E}-09$ & $6.66 \mathrm{E}-09$ & $6.96 \mathrm{E}-09$ & 7.65E-09 & $9.40 \mathrm{E}-09$ & $1.03 \mathrm{E}-08$ & $1.13 \mathrm{E}-08$ & $1.35 \mathrm{E}-08$ \\
\hline SC 46 & $8.02 \mathrm{E}-07$ & $7.96 \mathrm{E}-07$ & $7.89 \mathrm{E}-07$ & $7.39 \mathrm{E}-07$ & $6.26 \mathrm{E}-07$ & $3.81 \mathrm{E}-07$ & $2.74 \mathrm{E}-07$ & $1.81 \mathrm{E}-07$ & $4.08 \mathrm{E}-08$ \\
\hline $\mathrm{SC} 46 \mathrm{M}$ & $1.62 \mathrm{E}-16$ & $0.00 \mathrm{E}+00$ & $0.00 \mathrm{E}+00$ & $0.00 \mathrm{E}+00$ & $0.00 \mathrm{E}+00$ & $0.00 \mathrm{E}+00$ & $0.00 \mathrm{E}+00$ & $0.00 \mathrm{E}+00$ & $0.00 \mathrm{E}+00$ \\
\hline SC 47 & $8.50 \mathrm{E}-08$ & $6.91 \mathrm{E}-08$ & $5.62 \mathrm{E}-08$ & $1.08 \mathrm{E}-08$ & $1.72 \mathrm{E}-10$ & $7.13 \mathrm{E}-16$ & $2.14 \mathrm{E}-19$ & $2.14 \mathrm{E}-23$ & $1.77 \mathrm{E}-35$ \\
\hline
\end{tabular}


BSU-8242 3 DPA As-Run Physics Analysis

\begin{tabular}{|c|c|c|c|c|c|c|c|c|c|}
\hline \multicolumn{10}{|c|}{ ACTIVATION PRODUCTS (grams) } \\
\hline ISOTOPE & EOC & 1 Day & 2 Days & 10 Days & 30 Days & 90 Days & 130 Days & 180 Days & 360 Days \\
\hline $\mathrm{SC} 48$ & $3 \mathrm{E}-09$ & 3.03E-09 & $2.07 \mathrm{E}-09$ & 94E-11 & $5.00 \mathrm{E}-14$ & $6.36 \mathrm{E}-24$ & 30 & $9.12 \mathrm{E}-39$ & 0.00 \\
\hline SC 49 & $23 \mathrm{E}-11$ & $2.67 \mathrm{E}-18$ & $7.72 \mathrm{E}-26$ & $0.00 \mathrm{E}+00$ & $0.00 \mathrm{E}+00$ & $0.00 \mathrm{E}+00$ & $0.00 \mathrm{E}+00$ & $0.00 \mathrm{E}+00$ & $0.00 \mathrm{E}+00$ \\
\hline SC 50 & $3 \mathrm{E}-14$ & $0.00 \mathrm{E}+00$ & $0.00 \mathrm{E}+00$ & $0.00 \mathrm{E}+00$ & $0.00 \mathrm{E}+00$ & $0.00 \mathrm{E}+00$ & $0.00 \mathrm{E}+00$ & $0.00 \mathrm{E}+00$ & $0.00 \mathrm{E}+00$ \\
\hline TI 46 & $3.20 \mathrm{E}-02$ & $3.20 \mathrm{E}-02$ & $3.20 \mathrm{E}-02$ & $3.20 \mathrm{E}-02$ & $3.20 \mathrm{E}-02$ & $3.20 \mathrm{E}-02$ & $3.20 \mathrm{E}-02$ & $3.20 \mathrm{E}-02$ & $3.20 \mathrm{E}-02$ \\
\hline TI 47 & $2.95 \mathrm{E}-02$ & $2.95 \mathrm{E}-02$ & $2.95 \mathrm{E}-02$ & $2.95 \mathrm{E}-02$ & $2.95 \mathrm{E}-02$ & $2.95 \mathrm{E}-02$ & $2.95 \mathrm{E}-02$ & $2.95 \mathrm{E}-02$ & $2.95 \mathrm{E}-02$ \\
\hline TI 48 & $2.95 \mathrm{E}-01$ & $2.95 \mathrm{E}-01$ & $2.95 \mathrm{E}-01$ & $2.95 \mathrm{E}-01$ & $2.95 \mathrm{E}-01$ & & & & \\
\hline TI 49 & & & & & & & & & \\
\hline TI 50 & & & $\mathrm{E}-02$ & -02 & $\mathrm{E}-02$ & $2.22 \mathrm{E}-02$ & $2 \mathrm{E}-02$ & $2.22 \mathrm{E}-02$ & $.22 \mathrm{E}-02$ \\
\hline TI 51 & E-10 & $0.00 \mathrm{E}+00$ & $0.00 \mathrm{E}+00$ & $0.00 \mathrm{E}+00$ & $0.00 \mathrm{E}+00$ & $0.00 \mathrm{E}+00$ & $0.00 \mathrm{E}+00$ & $0.00 \mathrm{E}+00$ & $0.00 \mathrm{E}+00$ \\
\hline V 50 & $6.24 \mathrm{E}-04$ & $6.24 \mathrm{E}-04$ & $6.24 \mathrm{E}-04$ & $6.24 \mathrm{E}-04$ & $6.24 \mathrm{E}-04$ & $6.24 \mathrm{E}-04$ & $6.24 \mathrm{E}-04$ & $6.24 \mathrm{E}-04$ & $6.24 \mathrm{E}-04$ \\
\hline V 51 & $1.04 \mathrm{E}-01$ & $1.04 \mathrm{E}-01$ & 1.05E-01 & 1.09E-01 & 1.16 & 125 & 01 & $1.28 \mathrm{I}$ & $1.28 \mathrm{E}-01$ \\
\hline V 52 & & $0.00 \mathrm{E}+00$ & $0.00 \mathrm{E}+00$ & $0.00 \mathrm{E}+00$ & $0.00 \mathrm{E}+00$ & $0.00 \mathrm{E}+00$ & $0.00 \mathrm{E}+00$ & $0.00 \mathrm{E}+00$ & $0.00 \mathrm{E}+00$ \\
\hline V 53 & E-11 & $0.00 \mathrm{E}+00$ & $\mathrm{E}+00$ & $0.00 \mathrm{E}+00$ & $0.00 \mathrm{E}+00$ & $0.00 \mathrm{E}+00$ & $0.00 \mathrm{E}+00$ & $0.00 \mathrm{E}+00$ & $00 \mathrm{E}+00$ \\
\hline V 54 & -13 & $0.00 \mathrm{E}+00$ & $0.00 \mathrm{E}+00$ & $0.00 \mathrm{E}+00$ & $0.00 \mathrm{E}+00$ & $0.00 \mathrm{E}+00$ & $0.00 \mathrm{E}+00$ & $0.00 \mathrm{E}+00$ & $0.00 \mathrm{E}+00$ \\
\hline CR 50 & $5.30 \mathrm{E}+00$ & $5.30 \mathrm{E}+00$ & $5.30 \mathrm{E}+00$ & $5.30 \mathrm{E}+00$ & $5.30 \mathrm{E}+00$ & $5.30 \mathrm{E}+00$ & $5.30 \mathrm{E}+00$ & $5.30 \mathrm{E}+00$ & $5.30 \mathrm{E}+00$ \\
\hline CR 51 & $2.41 \mathrm{E}-02$ & $2.35 \mathrm{E}-02$ & $2.29 \mathrm{E}-02$ & $1.88 \mathrm{E}-02$ & 1.14E-02 & $2.54 \mathrm{E}-03$ & $9.33 \mathrm{E}-04$ & 2.67E-04 & $2.96 \mathrm{E}-06$ \\
\hline CR 52 & $1.08 \mathrm{E}+02$ & $1.08 \mathrm{E}+02$ & $1.08 \mathrm{E}+02$ & $1.08 \mathrm{E}+02$ & $1.08 \mathrm{E}+02$ & $1.08 \mathrm{E}+02$ & $1.08 \mathrm{E}+02$ & $1.08 \mathrm{E}+02$ & $1.08 \mathrm{E}+02$ \\
\hline CR 53 & & & & & & & & & $1.28 \mathrm{E}+01$ \\
\hline CR 54 & $\mathrm{E}+00$ & $8+00$ & $3.52 \mathrm{E}+00$ & $3.52 \mathrm{E}+00$ & $3.52 \mathrm{E}+00$ & $3.52 \mathrm{E}+00$ & $3.52 \mathrm{E}+00$ & $3.52 \mathrm{E}+00$ & $3.52 \mathrm{E}+00$ \\
\hline CR 55 & $3.79 \mathrm{E}-08$ & $0.00 \mathrm{E}+00$ & $0.00 \mathrm{E}+00$ & $0.00 \mathrm{E}+00$ & $0.00 \mathrm{E}+00$ & $0.00 \mathrm{E}+00$ & $0.00 \mathrm{E}+00$ & $0.00 \mathrm{E}+00$ & $0.00 \mathrm{E}+00$ \\
\hline MN 54 & $3.27 \mathrm{E}-03$ & $3.26 \mathrm{E}-03$ & $3.25 \mathrm{E}-03$ & 3.20E-03 & 3.06E-03 & $2.68 \mathrm{E}-03$ & $2.45 \mathrm{E}-03$ & $2.19 \mathrm{E}-03$ & $1.47 \mathrm{E}-03$ \\
\hline MN 55 & & $1.35 \mathrm{E}+00$ & & $1.35 \mathrm{E}+00$ & $1.35 \mathrm{E}+00$ & $1.36 \mathrm{E}+00$ & $1.36 \mathrm{E}+00$ & $1.36 \mathrm{E}+00$ & $1.36 \mathrm{E}+00$ \\
\hline MN 56 & & & & & & & $0.00 \mathrm{E}+00$ & $0.00 \mathrm{E}+00$ & $0.00 \mathrm{E}+00$ \\
\hline MN 57 & $1.15 \mathrm{E}-10$ & $0.00 \mathrm{E}+00$ & $0.00 \mathrm{E}+00$ & $0.00 \mathrm{E}+00$ & $0.00 \mathrm{E}+00$ & $0.00 \mathrm{E}+00$ & $0.00 \mathrm{E}+00$ & $0.00 \mathrm{E}+00$ & $0.00 \mathrm{E}+00$ \\
\hline MN 58 & $2.37 \mathrm{E}-13$ & $0.00 \mathrm{E}+00$ & $0.00 \mathrm{E}+00$ & $0.00 \mathrm{E}+00$ & $0.00 \mathrm{E}+00$ & $0.00 \mathrm{E}+00$ & $0.00 \mathrm{E}+00$ & $0.00 \mathrm{E}+00$ & $0.00 \mathrm{E}+00$ \\
\hline FE 54 & $1.43 \mathrm{E}+01$ & $1.43 \mathrm{E}+01$ & $1.43 \mathrm{E}+01$ & $1.43 \mathrm{E}+01$ & $1.43 \mathrm{E}+01$ & $1.43 \mathrm{E}+01$ & $1.43 \mathrm{E}+01$ & $1.43 \mathrm{E}+01$ & $1.43 \mathrm{E}+01$ \\
\hline
\end{tabular}


BSU-8242 3 DPA As-Run Physics Analysis

\begin{tabular}{|c|c|c|c|c|c|c|c|c|c|}
\hline \multicolumn{10}{|c|}{ ACTIVATION PRODUCTS (grams) } \\
\hline ISOTOPE & EOC & 1 Day & 2 Days & 10 Days & 30 Days & 90 Days & 130 Days & 180 Days & 360 Days \\
\hline FE 55 & $4.60 \mathrm{E}-02$ & $4.59 \mathrm{E}-02$ & $4.59 \mathrm{E}-02$ & $4.56 \mathrm{E}-02$ & $4.50 \mathrm{E}-02$ & 4.31E-02 & 4.18E-02 & 4.03E-02 & $3.54 \mathrm{E}-02$ \\
\hline FE 56 & $2.34 \mathrm{E}+02$ & $2.34 \mathrm{E}+02$ & $2.34 \mathrm{E}+02$ & $2.34 \mathrm{E}+02$ & $2.34 \mathrm{E}+02$ & $2.34 \mathrm{E}+02$ & $2.34 \mathrm{E}+02$ & $2.34 \mathrm{E}+02$ & $2.34 \mathrm{E}+02$ \\
\hline FE 57 & $6.60 \mathrm{E}+00$ & $6.60 \mathrm{E}+00$ & $6.60 \mathrm{E}+00$ & $6.60 \mathrm{E}+00$ & $6.60 \mathrm{E}+00$ & $6.60 \mathrm{E}+00$ & $6.60 \mathrm{E}+00$ & $6.60 \mathrm{E}+00$ & $6.60 \mathrm{E}+00$ \\
\hline FE 58 & $8.06 \mathrm{E}-01$ & $8.06 \mathrm{E}-01$ & $8.06 \mathrm{E}-01$ & 8.07E-01 & $8.08 \mathrm{E}-01$ & $8.10 \mathrm{E}-01$ & $8.11 \mathrm{E}-01$ & $8.12 \mathrm{E}-01$ & $8.13 \mathrm{E}-01$ \\
\hline FE 59 & $4.30 \mathrm{E}-04$ & $4.24 \mathrm{E}-04$ & 4.17E-04 & $3.69 \mathrm{E}-04$ & $2.71 \mathrm{E}-04$ & $1.08 \mathrm{E}-04$ & $5.81 \mathrm{E}-05$ & $2.69 \mathrm{E}-05$ & $1.68 \mathrm{E}-06$ \\
\hline CO 58 & $6.64 \mathrm{E}-03$ & $6.57 \mathrm{E}-03$ & $6.51 \mathrm{E}-03$ & $6.02 \mathrm{E}-03$ & $4.95 \mathrm{E}-03$ & $2.75 \mathrm{E}-03$ & $1.86 \mathrm{E}-03$ & $1.14 \mathrm{E}-03$ & $1.95 \mathrm{E}-04$ \\
\hline CO 59 & $1.53 \mathrm{E}+00$ & $1.53 \mathrm{E}+00$ & $1.53 \mathrm{E}+00$ & $1.53 \mathrm{E}+00$ & $1.53 \mathrm{E}+00$ & $1.53 \mathrm{E}+00$ & $1.53 \mathrm{E}+00$ & $1.53 \mathrm{E}+00$ & $1.53 \mathrm{E}+00$ \\
\hline $\mathrm{CO} 60$ & $1.16 \mathrm{E}-01$ & $1.16 \mathrm{E}-01$ & $1.16 \mathrm{E}-01$ & $1.15 \mathrm{E}-01$ & $1.15 \mathrm{E}-01$ & $1.12 \mathrm{E}-01$ & $1.11 \mathrm{E}-01$ & $1.09 \mathrm{E}-01$ & $1.02 \mathrm{E}-01$ \\
\hline $\mathrm{CO} 60 \mathrm{M}$ & $3.76 \mathrm{E}-06$ & $0.00 \mathrm{E}+00$ & $0.00 \mathrm{E}+00$ & $0.00 \mathrm{E}+00$ & $0.00 \mathrm{E}+00$ & $0.00 \mathrm{E}+00$ & $0.00 \mathrm{E}+00$ & $0.00 \mathrm{E}+00$ & $0.00 \mathrm{E}+00$ \\
\hline $\mathrm{CO} 61$ & $2.88 \mathrm{E}-07$ & $1.21 \mathrm{E}-11$ & $5.04 \mathrm{E}-16$ & $0.00 \mathrm{E}+00$ & $0.00 \mathrm{E}+00$ & $0.00 \mathrm{E}+00$ & $0.00 \mathrm{E}+00$ & $0.00 \mathrm{E}+00$ & $0.00 \mathrm{E}+00$ \\
\hline $\mathrm{CO} 62$ & $3.39 \mathrm{E}-11$ & $0.00 \mathrm{E}+00$ & $0.00 \mathrm{E}+00$ & $0.00 \mathrm{E}+00$ & $0.00 \mathrm{E}+00$ & $0.00 \mathrm{E}+00$ & $0.00 \mathrm{E}+00$ & $0.00 \mathrm{E}+00$ & $0.00 \mathrm{E}+00$ \\
\hline NI 58 & $1.42 \mathrm{E}+02$ & $1.42 \mathrm{E}+02$ & $1.42 \mathrm{E}+02$ & $1.42 \mathrm{E}+02$ & $1.42 \mathrm{E}+02$ & $1.42 \mathrm{E}+02$ & $1.42 \mathrm{E}+02$ & $1.42 \mathrm{E}+02$ & $1.42 \mathrm{E}+02$ \\
\hline NI 59 & $1.02 \mathrm{E}+00$ & $1.02 \mathrm{E}+00$ & $1.02 \mathrm{E}+00$ & $1.02 \mathrm{E}+00$ & $1.02 \mathrm{E}+00$ & $1.02 \mathrm{E}+00$ & $1.02 \mathrm{E}+00$ & $1.02 \mathrm{E}+00$ & $1.02 \mathrm{E}+00$ \\
\hline NI 60 & $5.63 \mathrm{E}+01$ & $5.63 \mathrm{E}+01$ & $5.63 \mathrm{E}+01$ & $5.63 \mathrm{E}+01$ & $5.63 \mathrm{E}+01$ & $5.63 \mathrm{E}+01$ & $5.63 \mathrm{E}+01$ & $5.63 \mathrm{E}+01$ & $5.63 \mathrm{E}+01$ \\
\hline NI 61 & $2.71 \mathrm{E}+00$ & $2.71 \mathrm{E}+00$ & $2.71 \mathrm{E}+00$ & $2.71 \mathrm{E}+00$ & $2.71 \mathrm{E}+00$ & $2.71 \mathrm{E}+00$ & $2.71 \mathrm{E}+00$ & $2.71 \mathrm{E}+00$ & $2.71 \mathrm{E}+00$ \\
\hline NI 62 & $7.86 \mathrm{E}+00$ & $7.86 \mathrm{E}+00$ & $7.86 \mathrm{E}+00$ & $7.86 \mathrm{E}+00$ & $7.86 \mathrm{E}+00$ & $7.86 \mathrm{E}+00$ & $7.86 \mathrm{E}+00$ & $7.86 \mathrm{E}+00$ & $7.86 \mathrm{E}+00$ \\
\hline NI 63 & $1.65 \mathrm{E}-01$ & $1.65 \mathrm{E}-01$ & $1.65 \mathrm{E}-01$ & $1.65 \mathrm{E}-01$ & $1.65 \mathrm{E}-01$ & $1.65 \mathrm{E}-01$ & $1.65 \mathrm{E}-01$ & $1.65 \mathrm{E}-01$ & $1.64 \mathrm{E}-01$ \\
\hline NI 64 & $2.10 \mathrm{E}+00$ & $2.10 \mathrm{E}+00$ & $2.10 \mathrm{E}+00$ & $2.10 \mathrm{E}+00$ & $2.10 \mathrm{E}+00$ & $2.10 \mathrm{E}+00$ & $2.10 \mathrm{E}+00$ & $2.10 \mathrm{E}+00$ & $2.10 \mathrm{E}+00$ \\
\hline NI 65 & $4.20 \mathrm{E}-06$ & $5.70 \mathrm{E}-09$ & $7.74 \mathrm{E}-12$ & $0.00 \mathrm{E}+00$ & $0.00 \mathrm{E}+00$ & $0.00 \mathrm{E}+00$ & $0.00 \mathrm{E}+00$ & $0.00 \mathrm{E}+00$ & $0.00 \mathrm{E}+00$ \\
\hline NI 66 & $2.76 \mathrm{E}-09$ & $2.04 \mathrm{E}-09$ & $1.50 \mathrm{E}-09$ & $1.31 \mathrm{E}-10$ & $2.97 \mathrm{E}-13$ & $3.43 \mathrm{E}-21$ & $1.75 \mathrm{E}-26$ & $4.25 \mathrm{E}-33$ & $0.00 \mathrm{E}+00$ \\
\hline CU 63 & $7.26 \mathrm{E}-01$ & $7.26 \mathrm{E}-01$ & $7.26 \mathrm{E}-01$ & $7.26 \mathrm{E}-01$ & $7.26 \mathrm{E}-01$ & $7.26 \mathrm{E}-01$ & $7.26 \mathrm{E}-01$ & 7.27E-01 & 7.27E-01 \\
\hline CU 64 & $2.41 \mathrm{E}-05$ & $6.49 \mathrm{E}-06$ & $1.75 \mathrm{E}-06$ & $4.92 \mathrm{E}-11$ & $2.06 \mathrm{E}-22$ & $0.00 \mathrm{E}+00$ & $0.00 \mathrm{E}+00$ & $0.00 \mathrm{E}+00$ & $0.00 \mathrm{E}+00$ \\
\hline CU 65 & $3.39 \mathrm{E}-01$ & $3.39 \mathrm{E}-01$ & $3.39 \mathrm{E}-01$ & $3.39 \mathrm{E}-01$ & $3.39 \mathrm{E}-01$ & $3.39 \mathrm{E}-01$ & $3.39 \mathrm{E}-01$ & $3.39 \mathrm{E}-01$ & $3.39 \mathrm{E}-01$ \\
\hline CU 66 & $3.64 \mathrm{E}-08$ & $3.17 \mathrm{E}-12$ & $2.34 \mathrm{E}-12$ & $2.05 \mathrm{E}-13$ & $4.63 \mathrm{E}-16$ & $5.34 \mathrm{E}-24$ & $2.73 \mathrm{E}-29$ & $6.63 \mathrm{E}-36$ & $0.00 \mathrm{E}+00$ \\
\hline CU 67 & $1.50 \mathrm{E}-10$ & $1.15 \mathrm{E}-10$ & $8.79 \mathrm{E}-11$ & $1.02 \mathrm{E}-11$ & $4.72 \mathrm{E}-14$ & $4.64 \mathrm{E}-21$ & $9.88 \mathrm{E}-26$ & $1.43 \mathrm{E}-31$ & $0.00 \mathrm{E}+00$ \\
\hline ZN 64 & $2.11 \mathrm{E}-03$ & $2.12 \mathrm{E}-03$ & $2.12 \mathrm{E}-03$ & $2.12 \mathrm{E}-03$ & $2.12 \mathrm{E}-03$ & $2.12 \mathrm{E}-03$ & $2.12 \mathrm{E}-03$ & $2.12 \mathrm{E}-03$ & $2.12 \mathrm{E}-03$ \\
\hline
\end{tabular}


BSU-8242 3 DPA As-Run Physics Analysis

\begin{tabular}{|c|c|c|c|c|c|c|c|c|c|}
\hline \multicolumn{10}{|c|}{ ACTIVATION PRODUCTS (grams) } \\
\hline ISOTOPE & EOC & 1 Day & 2 Days & 10 Days & 30 Days & 90 Days & 130 Days & 180 Days & 360 Days \\
\hline ZN 65 & $1.32 \mathrm{E}-06$ & $1.32 \mathrm{E}-06$ & $1.31 \mathrm{E}-06$ & $1.28 \mathrm{E}-06$ & $1.21 \mathrm{E}-06$ & $1.02 \mathrm{E}-06$ & $9.11 \mathrm{E}-07$ & $7.90 \mathrm{E}-07$ & 4.74E-07 \\
\hline ZN 66 & $1.28 \mathrm{E}-03$ & $1.28 \mathrm{E}-03$ & $1.28 \mathrm{E}-03$ & $1.28 \mathrm{E}-03$ & $1.28 \mathrm{E}-03$ & $1.28 \mathrm{E}-03$ & $1.28 \mathrm{E}-03$ & $1.28 \mathrm{E}-03$ & $1.28 \mathrm{E}-03$ \\
\hline $\mathrm{ZN} 67$ & $9.11 \mathrm{E}-07$ & $9.11 \mathrm{E}-07$ & $9.11 \mathrm{E}-07$ & $9.11 \mathrm{E}-07$ & $9.11 \mathrm{E}-07$ & $9.11 \mathrm{E}-07$ & $9.11 \mathrm{E}-07$ & $9.11 \mathrm{E}-07$ & $9.11 \mathrm{E}-07$ \\
\hline ZN 68 & $5.16 \mathrm{E}-09$ & $5.16 \mathrm{E}-09$ & $5.16 \mathrm{E}-09$ & $5.16 \mathrm{E}-09$ & $5.16 \mathrm{E}-09$ & $5.16 \mathrm{E}-09$ & $5.16 \mathrm{E}-09$ & $5.16 \mathrm{E}-09$ & $5.16 \mathrm{E}-09$ \\
\hline ZN 69 & $4.42 \mathrm{E}-15$ & $9.52 \mathrm{E}-17$ & $2.84 \mathrm{E}-17$ & $1.79 \mathrm{E}-21$ & $5.67 \mathrm{E}-32$ & $0.00 \mathrm{E}+00$ & $0.00 \mathrm{E}+00$ & $0.00 \mathrm{E}+00$ & $0.00 \mathrm{E}+00$ \\
\hline $\mathrm{ZN} \mathrm{69M}$ & $4.30 \mathrm{E}-15$ & $1.28 \mathrm{E}-15$ & $3.84 \mathrm{E}-16$ & $2.42 \mathrm{E}-20$ & $7.65 \mathrm{E}-31$ & $0.00 \mathrm{E}+00$ & $0.00 \mathrm{E}+00$ & $0.00 \mathrm{E}+00$ & $0.00 \mathrm{E}+00$ \\
\hline GA 69 & $3.49 \mathrm{E}-12$ & $3.50 \mathrm{E}-12$ & $3.50 \mathrm{E}-12$ & $3.50 \mathrm{E}-12$ & $3.50 \mathrm{E}-12$ & $3.50 \mathrm{E}-12$ & $3.50 \mathrm{E}-12$ & $3.50 \mathrm{E}-12$ & $3.50 \mathrm{E}-12$ \\
\hline GE 70 & $5.87 \mathrm{E}-15$ & $5.87 \mathrm{E}-15$ & $5.87 \mathrm{E}-15$ & $5.87 \mathrm{E}-15$ & $5.87 \mathrm{E}-15$ & $5.87 \mathrm{E}-15$ & $5.87 \mathrm{E}-15$ & $5.87 \mathrm{E}-15$ & $5.87 \mathrm{E}-15$ \\
\hline SR 87 & $8.38 \mathrm{E}-15$ & $8.38 \mathrm{E}-15$ & $8.38 \mathrm{E}-15$ & $8.38 \mathrm{E}-15$ & $8.38 \mathrm{E}-15$ & $8.38 \mathrm{E}-15$ & $8.38 \mathrm{E}-15$ & $8.38 \mathrm{E}-15$ & $8.38 \mathrm{E}-15$ \\
\hline SR 88 & $1.04 \mathrm{E}-11$ & $1.04 \mathrm{E}-11$ & $1.04 \mathrm{E}-11$ & $1.04 \mathrm{E}-11$ & $1.04 \mathrm{E}-11$ & $1.04 \mathrm{E}-11$ & $1.04 \mathrm{E}-11$ & $1.04 \mathrm{E}-11$ & $1.04 \mathrm{E}-11$ \\
\hline SR 89 & $5.42 \mathrm{E}-12$ & $5.34 \mathrm{E}-12$ & $5.27 \mathrm{E}-12$ & $4.72 \mathrm{E}-12$ & $3.59 \mathrm{E}-12$ & $1.58 \mathrm{E}-12$ & $9.09 \mathrm{E}-13$ & $4.58 \mathrm{E}-13$ & $3.87 \mathrm{E}-14$ \\
\hline SR 90 & $1.46 \mathrm{E}-12$ & $1.46 \mathrm{E}-12$ & $1.46 \mathrm{E}-12$ & $1.46 \mathrm{E}-12$ & $1.45 \mathrm{E}-12$ & $1.45 \mathrm{E}-12$ & $1.44 \mathrm{E}-12$ & $1.44 \mathrm{E}-12$ & $1.42 \mathrm{E}-12$ \\
\hline SR 91 & $1.32 \mathrm{E}-15$ & $2.29 \mathrm{E}-16$ & $3.98 \mathrm{E}-17$ & $3.28 \mathrm{E}-23$ & $2.02 \mathrm{E}-38$ & $0.00 \mathrm{E}+00$ & $0.00 \mathrm{E}+00$ & $0.00 \mathrm{E}+00$ & $0.00 \mathrm{E}+00$ \\
\hline Y 89 & $2.10 \mathrm{E}-07$ & $2.11 \mathrm{E}-07$ & $2.11 \mathrm{E}-07$ & $2.14 \mathrm{E}-07$ & $2.15 \mathrm{E}-07$ & $2.15 \mathrm{E}-07$ & $2.15 \mathrm{E}-07$ & $2.15 \mathrm{E}-07$ & $2.15 \mathrm{E}-07$ \\
\hline Y 89M & $3.20 \mathrm{E}-13$ & $2.59 \mathrm{E}-13$ & $2.09 \mathrm{E}-13$ & $3.84 \mathrm{E}-14$ & $5.52 \mathrm{E}-16$ & $1.64 \mathrm{E}-21$ & $3.40 \mathrm{E}-25$ & $8.45 \mathrm{E}-30$ & $0.00 \mathrm{E}+00$ \\
\hline Y 90 & $2.18 \mathrm{E}-08$ & $1.68 \mathrm{E}-08$ & $1.30 \mathrm{E}-08$ & $1.62 \mathrm{E}-09$ & 8.97E-12 & $3.65 \mathrm{E}-16$ & $3.62 \mathrm{E}-16$ & $3.61 \mathrm{E}-16$ & $3.57 \mathrm{E}-16$ \\
\hline Y 90M & $3.48 \mathrm{E}-16$ & $1.63 \mathrm{E}-18$ & $7.60 \mathrm{E}-21$ & $1.72 \mathrm{E}-39$ & $0.00 \mathrm{E}+00$ & $0.00 \mathrm{E}+00$ & $0.00 \mathrm{E}+00$ & $0.00 \mathrm{E}+00$ & $0.00 \mathrm{E}+00$ \\
\hline Y 91 & 4.70E-11 & $4.65 \mathrm{E}-11$ & $4.59 \mathrm{E}-11$ & $4.18 \mathrm{E}-11$ & $3.30 \mathrm{E}-11$ & $1.62 \mathrm{E}-11$ & $1.01 \mathrm{E}-11$ & $5.57 \mathrm{E}-12$ & $6.61 \mathrm{E}-13$ \\
\hline Y 92 & $6.29 \mathrm{E}-14$ & $5.72 \mathrm{E}-16$ & $5.20 \mathrm{E}-18$ & $2.42 \mathrm{E}-34$ & $0.00 \mathrm{E}+00$ & $0.00 \mathrm{E}+00$ & $0.00 \mathrm{E}+00$ & $0.00 \mathrm{E}+00$ & $0.00 \mathrm{E}+00$ \\
\hline Y 94 & $3.25 \mathrm{E}-17$ & $0.00 \mathrm{E}+00$ & $0.00 \mathrm{E}+00$ & $0.00 \mathrm{E}+00$ & $0.00 \mathrm{E}+00$ & $0.00 \mathrm{E}+00$ & $0.00 \mathrm{E}+00$ & $0.00 \mathrm{E}+00$ & $0.00 \mathrm{E}+00$ \\
\hline ZR 89 & $5.63 \mathrm{E}-09$ & $4.56 \mathrm{E}-09$ & $3.69 \mathrm{E}-09$ & $6.76 \mathrm{E}-10$ & $9.72 \mathrm{E}-12$ & $2.90 \mathrm{E}-17$ & $5.99 \mathrm{E}-21$ & $1.49 \mathrm{E}-25$ & $3.99 \mathrm{E}-42$ \\
\hline ZR 90 & $1.01 \mathrm{E}-06$ & $1.01 \mathrm{E}-06$ & $1.01 \mathrm{E}-06$ & $1.03 \mathrm{E}-06$ & $1.03 \mathrm{E}-06$ & $1.03 \mathrm{E}-06$ & $1.03 \mathrm{E}-06$ & $1.03 \mathrm{E}-06$ & $1.03 \mathrm{E}-06$ \\
\hline ZR 91 & $2.93 \mathrm{E}-06$ & $2.93 \mathrm{E}-06$ & $2.93 \mathrm{E}-06$ & $2.93 \mathrm{E}-06$ & $2.93 \mathrm{E}-06$ & $2.93 \mathrm{E}-06$ & $2.93 \mathrm{E}-06$ & $2.93 \mathrm{E}-06$ & $2.93 \mathrm{E}-06$ \\
\hline ZR 92 & $6.82 \mathrm{E}-05$ & $6.84 \mathrm{E}-05$ & $6.87 \mathrm{E}-05$ & $7.00 \mathrm{E}-05$ & $7.13 \mathrm{E}-05$ & $7.18 \mathrm{E}-05$ & $7.18 \mathrm{E}-05$ & $7.18 \mathrm{E}-05$ & $7.18 \mathrm{E}-05$ \\
\hline ZR 93 & $1.24 \mathrm{E}-05$ & $1.24 \mathrm{E}-05$ & $1.24 \mathrm{E}-05$ & $1.24 \mathrm{E}-05$ & $1.24 \mathrm{E}-05$ & $1.24 \mathrm{E}-05$ & $1.24 \mathrm{E}-05$ & $1.24 \mathrm{E}-05$ & $1.24 \mathrm{E}-05$ \\
\hline ZR 94 & $6.28 \mathrm{E}-06$ & $6.28 \mathrm{E}-06$ & $6.28 \mathrm{E}-06$ & $6.28 \mathrm{E}-06$ & $6.28 \mathrm{E}-06$ & $6.28 \mathrm{E}-06$ & $6.28 \mathrm{E}-06$ & $6.28 \mathrm{E}-06$ & $6.28 \mathrm{E}-06$ \\
\hline
\end{tabular}


BSU-8242 3 DPA As-Run Physics Analysis

\begin{tabular}{|c|c|c|c|c|c|c|c|c|c|}
\hline \multicolumn{10}{|c|}{ ACTIVATION PRODUCTS (grams) } \\
\hline ISOTOPE & EOC & 1 Day & 2 Days & 10 Days & 30 Days & 90 Days & 130 Days & 180 Days & 360 Days \\
\hline ZR 95 & $2.72 \mathrm{E}-07$ & $2.69 \mathrm{E}-07$ & $2.66 \mathrm{E}-07$ & $2.44 \mathrm{E}-07$ & $1.97 \mathrm{E}-07$ & $1.03 \mathrm{E}-07$ & $6.65 \mathrm{E}-08$ & $3.87 \mathrm{E}-08$ & $5.51 \mathrm{E}-09$ \\
\hline ZR 96 & $5.55 \mathrm{E}-10$ & $5.55 \mathrm{E}-10$ & $5.55 \mathrm{E}-10$ & $5.55 \mathrm{E}-10$ & $5.55 \mathrm{E}-10$ & $5.55 \mathrm{E}-10$ & $5.55 \mathrm{E}-10$ & $5.55 \mathrm{E}-10$ & $5.55 \mathrm{E}-10$ \\
\hline ZR 97 & $1.05 \mathrm{E}-10$ & $3.90 \mathrm{E}-11$ & $1.46 \mathrm{E}-11$ & $5.55 \mathrm{E}-15$ & $1.56 \mathrm{E}-23$ & $0.00 \mathrm{E}+00$ & $0.00 \mathrm{E}+00$ & $0.00 \mathrm{E}+00$ & $0.00 \mathrm{E}+00$ \\
\hline NB 92 & $3.55 \mathrm{E}-06$ & $3.32 \mathrm{E}-06$ & $3.10 \mathrm{E}-06$ & $1.80 \mathrm{E}-06$ & 4.59E-07 & 7.65E-09 & $5.00 \mathrm{E}-10$ & $1.65 \mathrm{E}-11$ & $7.66 \mathrm{E}-17$ \\
\hline NB 93 & $3.59 \mathrm{E}+00$ & $3.59 \mathrm{E}+00$ & $3.59 \mathrm{E}+00$ & $3.59 \mathrm{E}+00$ & $3.59 \mathrm{E}+00$ & $3.59 \mathrm{E}+00$ & $3.59 \mathrm{E}+00$ & $3.59 \mathrm{E}+00$ & $3.59 \mathrm{E}+00$ \\
\hline NB 93M & $2.50 \mathrm{E}-12$ & $2.51 \mathrm{E}-12$ & $2.53 \mathrm{E}-12$ & $2.64 \mathrm{E}-12$ & $2.93 \mathrm{E}-12$ & $3.78 \mathrm{E}-12$ & $4.34 \mathrm{E}-12$ & $5.04 \mathrm{E}-12$ & $7.52 \mathrm{E}-12$ \\
\hline NB 94 & $2.29 \mathrm{E}-02$ & $2.29 \mathrm{E}-02$ & $2.29 \mathrm{E}-02$ & $2.29 \mathrm{E}-02$ & $2.29 \mathrm{E}-02$ & $2.29 \mathrm{E}-02$ & $2.29 \mathrm{E}-02$ & $2.29 \mathrm{E}-02$ & $2.29 \mathrm{E}-02$ \\
\hline NB 95 & $2.95 \mathrm{E}-04$ & $2.89 \mathrm{E}-04$ & $2.83 \mathrm{E}-04$ & $2.42 \mathrm{E}-04$ & $1.63 \mathrm{E}-04$ & $5.00 \mathrm{E}-05$ & $2.28 \mathrm{E}-05$ & $8.51 \mathrm{E}-06$ & $2.50 \mathrm{E}-07$ \\
\hline NB 95M & $1.09 \mathrm{E}-10$ & $1.09 \mathrm{E}-10$ & $1.08 \mathrm{E}-10$ & $1.02 \mathrm{E}-10$ & $8.22 \mathrm{E}-11$ & $4.29 \mathrm{E}-11$ & $2.79 \mathrm{E}-11$ & $1.62 \mathrm{E}-11$ & $2.30 \mathrm{E}-12$ \\
\hline NB 96 & $4.45 \mathrm{E}-08$ & $2.18 \mathrm{E}-08$ & $1.07 \mathrm{E}-08$ & $3.58 \mathrm{E}-11$ & $2.32 \mathrm{E}-17$ & $6.33 \mathrm{E}-36$ & $0.00 \mathrm{E}+00$ & $0.00 \mathrm{E}+00$ & $0.00 \mathrm{E}+00$ \\
\hline NB 97 & $2.64 \mathrm{E}-10$ & $2.79 \mathrm{E}-12$ & $1.04 \mathrm{E}-12$ & $4.25 \mathrm{E}-16$ & $1.20 \mathrm{E}-24$ & $0.00 \mathrm{E}+00$ & $0.00 \mathrm{E}+00$ & $0.00 \mathrm{E}+00$ & $0.00 \mathrm{E}+00$ \\
\hline NB 97M & $9.75 \mathrm{E}-14$ & $3.65 \mathrm{E}-14$ & $1.36 \mathrm{E}-14$ & $5.18 \mathrm{E}-18$ & $1.46 \mathrm{E}-26$ & $0.00 \mathrm{E}+00$ & $0.00 \mathrm{E}+00$ & $0.00 \mathrm{E}+00$ & $0.00 \mathrm{E}+00$ \\
\hline NB 98 & $2.57 \mathrm{E}-14$ & $0.00 \mathrm{E}+00$ & $0.00 \mathrm{E}+00$ & $0.00 \mathrm{E}+00$ & $0.00 \mathrm{E}+00$ & $0.00 \mathrm{E}+00$ & $0.00 \mathrm{E}+00$ & $0.00 \mathrm{E}+00$ & $0.00 \mathrm{E}+00$ \\
\hline NB100 & $1.12 \mathrm{E}-15$ & $0.00 \mathrm{E}+00$ & $0.00 \mathrm{E}+00$ & $0.00 \mathrm{E}+00$ & $0.00 \mathrm{E}+00$ & $0.00 \mathrm{E}+00$ & $0.00 \mathrm{E}+00$ & $0.00 \mathrm{E}+00$ & $0.00 \mathrm{E}+00$ \\
\hline MO 92 & $1.47 \mathrm{E}+00$ & $1.47 \mathrm{E}+00$ & $1.47 \mathrm{E}+00$ & $1.47 \mathrm{E}+00$ & $1.47 \mathrm{E}+00$ & $1.47 \mathrm{E}+00$ & $1.47 \mathrm{E}+00$ & $1.47 \mathrm{E}+00$ & $1.47 \mathrm{E}+00$ \\
\hline MO 93M & $2.97 \mathrm{E}-08$ & $2.62 \mathrm{E}-09$ & $2.31 \mathrm{E}-10$ & $8.43 \mathrm{E}-19$ & $6.79 \mathrm{E}-40$ & $0.00 \mathrm{E}+00$ & $0.00 \mathrm{E}+00$ & $0.00 \mathrm{E}+00$ & $0.00 \mathrm{E}+00$ \\
\hline MO 93 & $4.00 \mathrm{E}-04$ & $4.00 \mathrm{E}-04$ & $4.00 \mathrm{E}-04$ & $4.00 \mathrm{E}-04$ & $4.00 \mathrm{E}-04$ & $4.00 \mathrm{E}-04$ & $4.00 \mathrm{E}-04$ & $4.00 \mathrm{E}-04$ & $4.00 \mathrm{E}-04$ \\
\hline MO 94 & $9.42 \mathrm{E}-01$ & $9.42 \mathrm{E}-01$ & $9.42 \mathrm{E}-01$ & $9.42 \mathrm{E}-01$ & $9.42 \mathrm{E}-01$ & $9.42 \mathrm{E}-01$ & $9.42 \mathrm{E}-01$ & $9.42 \mathrm{E}-01$ & $9.42 \mathrm{E}-01$ \\
\hline MO 95 & $1.52 \mathrm{E}+00$ & $1.52 \mathrm{E}+00$ & $1.52 \mathrm{E}+00$ & $1.52 \mathrm{E}+00$ & $1.52 \mathrm{E}+00$ & $1.52 \mathrm{E}+00$ & $1.52 \mathrm{E}+00$ & $1.52 \mathrm{E}+00$ & $1.52 \mathrm{E}+00$ \\
\hline MO 96 & $1.82 \mathrm{E}+00$ & $1.82 \mathrm{E}+00$ & $1.82 \mathrm{E}+00$ & $1.82 \mathrm{E}+00$ & $1.82 \mathrm{E}+00$ & $1.82 \mathrm{E}+00$ & $1.82 \mathrm{E}+00$ & $1.82 \mathrm{E}+00$ & $1.82 \mathrm{E}+00$ \\
\hline MO 97 & $1.01 \mathrm{E}+00$ & $1.01 \mathrm{E}+00$ & $1.01 \mathrm{E}+00$ & $1.01 \mathrm{E}+00$ & $1.01 \mathrm{E}+00$ & $1.01 \mathrm{E}+00$ & $1.01 \mathrm{E}+00$ & $1.01 \mathrm{E}+00$ & $1.01 \mathrm{E}+00$ \\
\hline MO 98 & $2.55 \mathrm{E}+00$ & $2.55 \mathrm{E}+00$ & $2.55 \mathrm{E}+00$ & $2.55 \mathrm{E}+00$ & $2.55 \mathrm{E}+00$ & $2.55 \mathrm{E}+00$ & $2.55 \mathrm{E}+00$ & $2.55 \mathrm{E}+00$ & $2.55 \mathrm{E}+00$ \\
\hline MO 99 & $2.13 \mathrm{E}-04$ & $1.66 \mathrm{E}-04$ & $1.29 \mathrm{E}-04$ & $1.72 \mathrm{E}-05$ & $1.11 \mathrm{E}-07$ & $3.00 \mathrm{E}-14$ & $1.26 \mathrm{E}-18$ & $4.22 \mathrm{E}-24$ & $1.39 \mathrm{E}-43$ \\
\hline MO100 & $1.03 \mathrm{E}+00$ & $1.03 \mathrm{E}+00$ & $1.03 \mathrm{E}+00$ & $1.03 \mathrm{E}+00$ & $1.03 \mathrm{E}+00$ & $1.03 \mathrm{E}+00$ & $1.03 \mathrm{E}+00$ & $1.03 \mathrm{E}+00$ & $1.03 \mathrm{E}+00$ \\
\hline MO101 & $2.00 \mathrm{E}-07$ & $0.00 \mathrm{E}+00$ & $0.00 \mathrm{E}+00$ & $0.00 \mathrm{E}+00$ & $0.00 \mathrm{E}+00$ & $0.00 \mathrm{E}+00$ & $0.00 \mathrm{E}+00$ & $0.00 \mathrm{E}+00$ & $0.00 \mathrm{E}+00$ \\
\hline TC 99 & $1.10 \mathrm{E}-03$ & $1.10 \mathrm{E}-03$ & $1.11 \mathrm{E}-03$ & $1.12 \mathrm{E}-03$ & $1.13 \mathrm{E}-03$ & $1.13 \mathrm{E}-03$ & $1.13 \mathrm{E}-03$ & $1.13 \mathrm{E}-03$ & $1.13 \mathrm{E}-03$ \\
\hline
\end{tabular}


BSU-8242 3 DPA As-Run Physics Analysis

\begin{tabular}{|c|c|c|c|c|c|c|c|c|c|}
\hline \multicolumn{10}{|c|}{ ACTIVATION PRODUCTS (grams) } \\
\hline ISOTOPE & EOC & 1 Day & 2 Days & 10 Days & 30 Days & 90 Days & 130 Days & 180 Days & 360 Days \\
\hline $\mathrm{TC} 100$ & $.36 \mathrm{E}-10$ & $.00 \mathrm{E}+00$ & $0.00 \mathrm{E}+00$ & $0.00 \mathrm{E}+00$ & $0.00 \mathrm{E}+00$ & $0.00 \mathrm{E}+00$ & $0.00 \mathrm{E}+00$ & $0.00 \mathrm{E}+00$ & $0.00 \mathrm{E}+00$ \\
\hline TC101 & $94 \mathrm{E}-07$ & $0.00 \mathrm{E}+00$ & $0.00 \mathrm{E}+00$ & $0.00 \mathrm{E}+00$ & $0.00 \mathrm{E}+00$ & $0.00 \mathrm{E}+00$ & $0.00 \mathrm{E}+00$ & $0.00 \mathrm{E}+00$ & $0.00 \mathrm{E}+00$ \\
\hline RU 99 & $72 \mathrm{E}-09$ & $1.73 \mathrm{E}-09$ & $1.74 \mathrm{E}-09$ & $1.82 \mathrm{E}-09$ & $2.02 \mathrm{E}-09$ & $2.62 \mathrm{E}-09$ & $3.03 \mathrm{E}-09$ & $3.53 \mathrm{E}-09$ & $5.33 \mathrm{E}-09$ \\
\hline RU100 & $8.22 \mathrm{E}-05$ & $8.22 \mathrm{E}-05$ & $8.22 \mathrm{E}-05$ & $8.22 \mathrm{E}-05$ & $8.22 \mathrm{E}-05$ & $8.22 \mathrm{E}-05$ & $8.22 \mathrm{E}-05$ & $8.22 \mathrm{E}-05$ & $8.22 \mathrm{E}-05$ \\
\hline RU101 & $2.40 \mathrm{E}-03$ & $2.40 \mathrm{E}-03$ & $2.40 \mathrm{E}-03$ & $2.40 \mathrm{E}-03$ & & & 2.40 & 2.40 & -03 \\
\hline RU102 & & & $5.73 \mathrm{E}-05$ & $5.73 \mathrm{E}-05$ & $5.73 \mathrm{E}-05$ & $5.73 \mathrm{E}-05$ & $5.73 \mathrm{E}-05$ & $5.73 \mathrm{E}-05$ & $.73 \mathrm{E}-05$ \\
\hline RU103 & & $3.92 \mathrm{E}-08$ & -08 & $\mathrm{E}-08$ & & E-09 & -09 & -09 & -11 \\
\hline RU104 & E-10 & -10 & $4.31 \mathrm{E}-10$ & $4.31 \mathrm{E}-10$ & 4.3 & $4.31 \mathrm{E}-10$ & $4.31 \mathrm{E}-10$ & $4.31 \mathrm{E}-10$ & $4.31 \mathrm{E}-10$ \\
\hline RU105 & $2.68 \mathrm{E}-15$ & $6.31 \mathrm{E}-17$ & $1.49 \mathrm{E}-18$ & $1.42 \mathrm{E}-31$ & $0.00 \mathrm{E}+00$ & $0.00 \mathrm{E}+00$ & $0.00 \mathrm{E}+00$ & $0.00 \mathrm{E}+00$ & $0.00 \mathrm{E}+00$ \\
\hline RH103 & 3.44E-09 & $3.51 \mathrm{E}-09$ & $3.58 \mathrm{E}-09$ & $4.08 \mathrm{E}-09$ & $5.07 \mathrm{E}-09$ & & 7.01E-09 & 7.24E-09 & 7.40E-09 \\
\hline RH104 & $8.58 \mathrm{E}-15$ & $0.00 \mathrm{E}+00$ & $0.00 \mathrm{E}+00$ & $0.00 \mathrm{E}+00$ & $0.00 \mathrm{E}+00$ & $0.00 \mathrm{E}+00$ & $0.00 \mathrm{E}+00$ & $0.00 \mathrm{E}+00$ & $0.00 \mathrm{E}+00$ \\
\hline RH104N & E-15 & $0.00 \mathrm{E}+00$ & $0.00 \mathrm{E}+00$ & $0.00 \mathrm{E}+00$ & $0.00 \mathrm{E}+00$ & $0.00 \mathrm{E}+00$ & $0.00 \mathrm{E}+00$ & $0.00 \mathrm{E}+00$ & $\underline{0 \mathrm{E}+00}$ \\
\hline RH105 & & & & 1.7 & 1.4 & 8.0 & 41 & $0.00 \mathrm{E}+00$ & $\mathrm{E}+00$ \\
\hline RH106M & $9.70 \mathrm{E}-17$ & $5.05 \mathrm{E}$ & $2.62 \mathrm{E}-23$ & $0.00 \mathrm{E}+00$ & $0.00 \mathrm{E}+00$ & $0.00 \mathrm{E}+00$ & $0.00 \mathrm{E}+00$ & $0.00 \mathrm{E}+00$ & $0.00 \mathrm{E}+00$ \\
\hline PD104 & $5.64 \mathrm{E}-10$ & $5.64 \mathrm{E}-10$ & $5.64 \mathrm{E}-10$ & $5.64 \mathrm{E}-10$ & $5.64 \mathrm{E}-10$ & $5.64 \mathrm{E}-10$ & $5.64 \mathrm{E}-10$ & $5.64 \mathrm{E}-10$ & $5.64 \mathrm{E}-10$ \\
\hline PD105 & $1.44 \mathrm{E}-12$ & $1.45 \mathrm{E}-12$ & $1.45 \mathrm{E}-12$ & & 1.46E-12 & $1.46 \mathrm{E}-12$ & $1.46 \mathrm{E}-12$ & 1.46E-12 & $1.46 \mathrm{E}-12$ \\
\hline & & & & & & & & & \\
\hline PD107 & & & 7.1 & -17 & 7.1 & 7.1 & 7.1 & -17 & $7.16 \mathrm{E}-17$ \\
\hline TM172 & $6.70 \mathrm{E}-15$ & $5.15 \mathrm{E}-15$ & $3.97 \mathrm{E}-15$ & $4.90 \mathrm{E}-16$ & $2.62 \mathrm{E}-18$ & $4.00 \mathrm{E}-25$ & $1.14 \mathrm{E}-29$ & $2.39 \mathrm{E}-35$ & $0.00 \mathrm{E}+00$ \\
\hline TM173 & $7.78 \mathrm{E}-16$ & 1.03E-16 & $1.37 \mathrm{E}-17$ & $1.33 \mathrm{E}-24$ & $3.88 \mathrm{E}-42$ & $0.00 \mathrm{E}+00$ & $0.00 \mathrm{E}+00$ & $0.00 \mathrm{E}+00$ & $0.00 \mathrm{E}+00$ \\
\hline YB172 & 1.67E-13 & $1.69 \mathrm{E}-13$ & $1.70 \mathrm{E}-13$ & $1.73 \mathrm{E}-13$ & $1.74 \mathrm{E}-13$ & $1.74 \mathrm{E}-13$ & $1.74 \mathrm{E}-13$ & $1.74 \mathrm{E}-13$ & $1.74 \mathrm{E}-13$ \\
\hline YB173 & $1.09 \mathrm{E}-13$ & $1.10 \mathrm{E}-13$ & & & $1.10 \mathrm{E}-13$ & $1.10 \mathrm{E}-13$ & $1.10 \mathrm{E}-13$ & $1.10 \mathrm{E}-13$ & $1.10 \mathrm{E}-13$ \\
\hline YB174 & $5.01 \mathrm{E}-15$ & $5.01 \mathrm{E}-15$ & $5.01 \mathrm{E}-15$ & $5.01 \mathrm{E}-15$ & $5.01 \mathrm{E}-15$ & $5.01 \mathrm{E}-15$ & $5.01 \mathrm{E}-15$ & $5.01 \mathrm{E}-15$ & $5.01 \mathrm{E}-15$ \\
\hline YB175 & 1.63E-17 & $1.38 \mathrm{E}-17$ & $1.17 \mathrm{E}-17$ & $3.12 \mathrm{E}-18$ & 1.14E-19 & $5.57 \mathrm{E}-24$ & $6.13 \mathrm{E}-24$ & $1.57 \mathrm{E}-27$ & $1.83 \mathrm{E}-40$ \\
\hline LU175 & $3.59 \mathrm{E}-06$ & $3.61 \mathrm{E}-06$ & $3.63 \mathrm{E}-06$ & $3.77 \mathrm{E}-06$ & $4.08 \mathrm{E}-06$ & $4.71 \mathrm{E}-06$ & $4.97 \mathrm{E}-06$ & $5.17 \mathrm{E}-06$ & $5.44 \mathrm{E}-06$ \\
\hline LU176 & $9.86 \mathrm{E}-08$ & $9.86 \mathrm{E}-08$ & $9.86 \mathrm{E}-08$ & $9.86 \mathrm{E}-08$ & $9.86 \mathrm{E}-08$ & $9.86 \mathrm{E}-08$ & $9.86 \mathrm{E}-08$ & $9.86 \mathrm{E}-08$ & $9.86 \mathrm{E}-08$ \\
\hline
\end{tabular}


BSU-8242 3 DPA As-Run Physics Analysis

\begin{tabular}{|c|c|c|c|c|c|c|c|c|c|}
\hline \multicolumn{10}{|c|}{ ACTIVATION PRODUCTS (grams) } \\
\hline ISOTOPE & EOC & 1 Day & 2 Days & 10 Days & 30 Days & 90 Days & 130 Days & 180 Days & 360 Days \\
\hline LU176M & $1.18 \mathrm{E}-09$ & $1.30 \mathrm{E}-11$ & $1.43 \mathrm{E}-13$ & $3.10 \mathrm{E}-29$ & $0.00 \mathrm{E}+00$ & $0.00 \mathrm{E}+00$ & $0.00 \mathrm{E}+00$ & $0.00 \mathrm{E}+00$ & $0.00 \mathrm{E}+00$ \\
\hline LU177 & $58 \mathrm{E}-08$ & $1.42 \mathrm{E}-08$ & $1.28 \mathrm{E}-08$ & $5.62 \mathrm{E}-09$ & $15 \mathrm{E}-10$ & $.62 \mathrm{E}-12$ & $.84 \mathrm{E}-12$ & $1.45 \mathrm{E}-12$ & $49 \mathrm{E}-13$ \\
\hline LU177M & $26 \mathrm{E}-10$ & $3.25 \mathrm{E}-10$ & 3.23E-10 & $3.12 \mathrm{E}-10$ & $2.85 \mathrm{E}-10$ & $2.18 \mathrm{E}-10$ & $1.82 \mathrm{E}-10$ & $1.46 \mathrm{E}-10$ & $6.52 \mathrm{E}-11$ \\
\hline HF174 & $6.32 \mathrm{E}-06$ & $6.32 \mathrm{E}-06$ & $6.32 \mathrm{E}-06$ & $6.32 \mathrm{E}-06$ & $6.32 \mathrm{E}-06$ & $6.32 \mathrm{E}-06$ & $6.32 \mathrm{E}-06$ & $6.32 \mathrm{E}-06$ & $6.32 \mathrm{E}-06$ \\
\hline HF175 & $1.90 \mathrm{E}-06$ & $1.88 \mathrm{E}-06$ & $1.86 \mathrm{E}-06$ & $1.72 \mathrm{E}-06$ & $1.41 \mathrm{E}-06$ & $7.78 \mathrm{E}-07$ & $5.24 \mathrm{E}-07$ & 3.19E-07 & $5.37 \mathrm{E}-08$ \\
\hline HF176 & $2.99 \mathrm{E}-04$ & $2.99 \mathrm{E}-04$ & $2.99 \mathrm{E}-04$ & $2.99 \mathrm{E}-04$ & & & & & \\
\hline & & & 8.51E-05 & $851 \mathrm{E} 05$ & 8.51E-05 & $8.51 \mathrm{E}-05$ & $8.51 \mathrm{E}-05$ & $8.51 \mathrm{E}-05$ & $8.51 \mathrm{E}-05$ \\
\hline & & & & & & -03 & & -03 & -03 \\
\hline HF178I & $5 \mathrm{E}-13$ & $0.00 \mathrm{E}+00$ & $0.00 \mathrm{E}+00$ & $0.00 \mathrm{E}+00$ & $0.00 \mathrm{E}+00$ & $0.00 \mathrm{E}+00$ & $0.00 \mathrm{E}+00$ & $0.00 \mathrm{E}+00$ & $0.00 \mathrm{E}+00$ \\
\hline HF179 & $2.51 \mathrm{E}-03$ & $2.51 \mathrm{E}-03$ & $2.51 \mathrm{E}-03$ & $2.51 \mathrm{E}-03$ & $2.51 \mathrm{E}-03$ & $2.51 \mathrm{E}-03$ & $2.51 \mathrm{E}-03$ & $2.51 \mathrm{E}-03$ & $2.51 \mathrm{E}-03$ \\
\hline HF179M & 1.62E-09 & $0.00 \mathrm{E}+00$ & $0.00 \mathrm{E}+00$ & $0.00 \mathrm{E}+00$ & $0.00 \mathrm{E}$ & $0.00 \mathrm{E}+00$ & $0.00 \mathrm{E}+00$ & & $0.00 \mathrm{E}+00$ \\
\hline HF180 & & & & & & & & & \\
\hline $\mathrm{HF} 1$ & & & & & +00 & $\mathrm{E}+00$ & +00 & $E+00$ & $\mathrm{E}+00$ \\
\hline HF181 & -05 & $2.68 \mathrm{E}-05$ & $2.64 \mathrm{E}-05$ & $2.31 \mathrm{E}-05$ & $1.67 \mathrm{E}-05$ & $6.26 \mathrm{E}-06$ & $3.25 \mathrm{E}-06$ & $1.44 \mathrm{E}-06$ & $7.57 \mathrm{E}-08$ \\
\hline HF182 & $8.83 \mathrm{E}-07$ & $8.83 \mathrm{E}-07$ & $8.83 \mathrm{E}-07$ & $8.83 \mathrm{E}-07$ & $8.83 \mathrm{E}-07$ & $8.83 \mathrm{E}-07$ & $8.83 \mathrm{E}-07$ & $8.83 \mathrm{E}-07$ & $8.83 \mathrm{E}-07$ \\
\hline TA180 & $1.07 \mathrm{E}-04$ & $1.07 \mathrm{E}-04$ & $1.07 \mathrm{E}-04$ & $1.07 \mathrm{E}-04$ & $1.07 \mathrm{E}-04$ & $1.07 \mathrm{E}-04$ & $1.07 \mathrm{E}-04$ & $1.07 \mathrm{E}-04$ & $1.07 \mathrm{E}-04$ \\
\hline TA181 & $2.05 \mathrm{E}+00$ & $2.05 \mathrm{E}+00$ & $2.05 \mathrm{E}+00$ & $2.05 \mathrm{E}+00$ & $2.05 \mathrm{E}+00$ & $2.05 \mathrm{E}+00$ & $2.05 \mathrm{E}+00$ & $2.05 \mathrm{E}+00$ & $2.05 \mathrm{E}+00$ \\
\hline & & & & & & & & & \\
\hline TA182N & & 0.0 & 0.0 & $0.00 \mathrm{E}+00$ & $0.00 \mathrm{E}+00$ & $0.00 \mathrm{E}+00$ & $0.00 \mathrm{E}+00$ & $0.00 \mathrm{E}+00$ & $0.00 \mathrm{E}+00$ \\
\hline TA183 & $2.43 \mathrm{E}-02$ & $2.12 \mathrm{E}-02$ & $1.85 \mathrm{E}-02$ & $6.23 \mathrm{E}-03$ & $4.11 \mathrm{E}-04$ & $1.18 \mathrm{E}-07$ & $5.15 \mathrm{E}-10$ & $5.75 \mathrm{E}-13$ & $1.37 \mathrm{E}-23$ \\
\hline W180 & $9.76 \mathrm{E}-18$ & $9.78 \mathrm{E}-18$ & 9.79E-18 & $9.89 \mathrm{E}-18$ & 1.01E-17 & $1.09 \mathrm{E}-17$ & $1.14 \mathrm{E}-17$ & $1.20 \mathrm{E}-17$ & $1.43 \mathrm{E}-17$ \\
\hline W182 & & & $9.86 \mathrm{E}-02$ & & & $1.20 \mathrm{E}-01$ & 1.27E-01 & 1.33E-01 & $1.45 \mathrm{E}-01$ \\
\hline W183M & & $0.00 \mathrm{E}+00$ & $0.00 \mathrm{E}+00$ & $0.00 \mathrm{E}+00$ & $0.00 \mathrm{E}+00$ & $0.00 \mathrm{E}+00$ & $0.00 \mathrm{E}+00$ & $0.00 \mathrm{E}+00$ & $0.00 \mathrm{E}+00$ \\
\hline W183 & $5.84 \mathrm{E}-01$ & $5.87 \mathrm{E}-01$ & $5.90 \mathrm{E}-01$ & $6.02 \mathrm{E}-01$ & $6.08 \mathrm{E}-01$ & $6.09 \mathrm{E}-01$ & $6.09 \mathrm{E}-01$ & $6.09 \mathrm{E}-01$ & $6.09 \mathrm{E}-01$ \\
\hline W184 & 4.24E-02 & 4.24E-02 & 4.24E-02 & 4.24E-02 & 4.24E-02 & $4.24 \mathrm{E}-02$ & 4.24E-02 & 4.24E-02 & $4.24 \mathrm{E}-02$ \\
\hline W185 & $7.04 \mathrm{E}-05$ & $6.98 \mathrm{E}-05$ & $6.92 \mathrm{E}-05$ & $6.42 \mathrm{E}-05$ & $5.34 \mathrm{E}-05$ & $3.07 \mathrm{E}-05$ & $2.12 \mathrm{E}-05$ & $1.34 \mathrm{E}-05$ & $2.54 \mathrm{E}-06$ \\
\hline
\end{tabular}




\begin{tabular}{|lrr|}
\hline TEM-10200-1, Rev. 11 & ENGINEERING CALCULATIONS AND ANALYSIS & ECAR- 4951, Rev. 1 \\
$11 / 20 / 2019$ & Page 73 of 104 \\
& BSU-8242 3 DPA As-Run Physics Analysis & \\
\hline
\end{tabular}

\begin{tabular}{|c|c|c|c|c|c|c|c|c|c|}
\hline \multicolumn{10}{|c|}{ ACTIVATION PRODUCTS (grams) } \\
\hline ISOTOPE & EOC & 1 Day & 2 Days & 10 Days & 30 Days & 90 Days & 130 Days & 180 Days & 360 Days \\
\hline $\mathrm{W} 185 \mathrm{M}$ & $3.39 \mathrm{E}-12$ & $0.00 \mathrm{E}+00$ & $0.00 \mathrm{E}+00$ & $0.00 \mathrm{E}+00$ & $0.00 \mathrm{E}+00$ & $0.00 \mathrm{E}+00$ & $0.00 \mathrm{E}+00$ & $0.00 \mathrm{E}+00$ & $0.00 \mathrm{E}+00$ \\
\hline W186 & $3.55 \mathrm{E}-07$ & $3.63 \mathrm{E}-07$ & $3.70 \mathrm{E}-07$ & $3.95 \mathrm{E}-07$ & $4.02 \mathrm{E}-07$ & $4.03 \mathrm{E}-07$ & $4.03 \mathrm{E}-07$ & $4.03 \mathrm{E}-07$ & $4.03 \mathrm{E}-07$ \\
\hline W187 & $6.88 \mathrm{E}-10$ & $3.43 \mathrm{E}-10$ & $1.71 \mathrm{E}-10$ & $6.52 \mathrm{E}-13$ & $5.87 \mathrm{E}-19$ & $4.28 \mathrm{E}-37$ & $0.00 \mathrm{E}+00$ & $0.00 \mathrm{E}+00$ & $0.00 \mathrm{E}+00$ \\
\hline W188 & $1.18 \mathrm{E}-10$ & $1.17 \mathrm{E}-10$ & $1.16 \mathrm{E}-10$ & $1.07 \mathrm{E}-10$ & $8.75 \mathrm{E}-11$ & $4.81 \mathrm{E}-11$ & $3.22 \mathrm{E}-11$ & $1.96 \mathrm{E}-11$ & $3.24 \mathrm{E}-12$ \\
\hline RE185 & $2.93 \mathrm{E}-05$ & $3.00 \mathrm{E}-05$ & $3.06 \mathrm{E}-05$ & $3.55 \mathrm{E}-05$ & $4.63 \mathrm{E}-05$ & $6.91 \mathrm{E}-05$ & $7.85 \mathrm{E}-05$ & $8.64 \mathrm{E}-05$ & $9.72 \mathrm{E}-05$ \\
\hline RE186 & $7.36 \mathrm{E}-07$ & $6.13 \mathrm{E}-07$ & $5.10 \mathrm{E}-07$ & $1.17 \mathrm{E}-07$ & $2.99 \mathrm{E}-09$ & $4.93 \mathrm{E}-14$ & $3.20 \mathrm{E}-17$ & $3.31 \mathrm{E}-21$ & $1.49 \mathrm{E}-35$ \\
\hline RE187 & 1.64E-08 & $1.67 \mathrm{E}-08$ & $1.69 \mathrm{E}-08$ & $1.71 \mathrm{E}-08$ & $1.71 \mathrm{E}-08$ & $1.71 \mathrm{E}-08$ & $1.71 \mathrm{E}-08$ & $1.71 \mathrm{E}-08$ & $1.71 \mathrm{E}-08$ \\
\hline RE188 & $2.10 \mathrm{E}-11$ & $8.76 \mathrm{E}-12$ & $4.03 \mathrm{E}-12$ & $1.10 \mathrm{E}-12$ & $9.02 \mathrm{E}-13$ & $4.95 \mathrm{E}-13$ & $3.32 \mathrm{E}-13$ & $2.02 \mathrm{E}-13$ & $3.34 \mathrm{E}-14$ \\
\hline RE188M & $3.55 \mathrm{E}-13$ & $0.00 \mathrm{E}+00$ & $0.00 \mathrm{E}+00$ & $0.00 \mathrm{E}+00$ & $0.00 \mathrm{E}+00$ & $0.00 \mathrm{E}+00$ & $0.00 \mathrm{E}+00$ & $0.00 \mathrm{E}+00$ & $0.00 \mathrm{E}+00$ \\
\hline RE189 & $4.99 \mathrm{E}-16$ & $2.52 \mathrm{E}-16$ & $1.27 \mathrm{E}-16$ & $5.31 \mathrm{E}-19$ & $6.01 \mathrm{E}-25$ & $7.95 \mathrm{E}-43$ & $0.00 \mathrm{E}+00$ & $0.00 \mathrm{E}+00$ & $0.00 \mathrm{E}+00$ \\
\hline OS186 & $5.35 \mathrm{E}-06$ & $5.47 \mathrm{E}-06$ & $5.56 \mathrm{E}-06$ & $5.93 \mathrm{E}-06$ & $6.04 \mathrm{E}-06$ & $6.04 \mathrm{E}-06$ & $6.04 \mathrm{E}-06$ & $6.04 \mathrm{E}-06$ & $6.04 \mathrm{E}-06$ \\
\hline OS188 & $5.94 \mathrm{E}-10$ & $6.07 \mathrm{E}-10$ & $6.13 \mathrm{E}-10$ & $6.25 \mathrm{E}-10$ & $6.45 \mathrm{E}-10$ & $6.84 \mathrm{E}-10$ & $7.00 \mathrm{E}-10$ & $7.13 \mathrm{E}-10$ & $7.30 \mathrm{E}-10$ \\
\hline OS189 & $4.67 \mathrm{E}-12$ & $4.67 \mathrm{E}-12$ & $4.67 \mathrm{E}-12$ & $4.67 \mathrm{E}-12$ & $4.67 \mathrm{E}-12$ & $4.67 \mathrm{E}-12$ & $4.67 \mathrm{E}-12$ & $4.67 \mathrm{E}-12$ & $4.67 \mathrm{E}-12$ \\
\hline OS190 & $1.83 \mathrm{E}-13$ & $1.83 \mathrm{E}-13$ & $1.83 \mathrm{E}-13$ & $1.83 \mathrm{E}-13$ & $1.83 \mathrm{E}-13$ & $1.83 \mathrm{E}-13$ & $1.83 \mathrm{E}-13$ & $1.83 \mathrm{E}-13$ & $1.83 \mathrm{E}-13$ \\
\hline OS191 & $3.01 \mathrm{E}-16$ & $2.99 \mathrm{E}-16$ & $2.88 \mathrm{E}-16$ & $2.02 \mathrm{E}-16$ & $8.22 \mathrm{E}-17$ & $5.52 \mathrm{E}-18$ & $9.13 \mathrm{E}-19$ & $9.63 \mathrm{E}-20$ & $2.93 \mathrm{E}-23$ \\
\hline OS191M & $1.57 \mathrm{E}-17$ & $4.35 \mathrm{E}-18$ & $1.21 \mathrm{E}-18$ & $4.34 \mathrm{E}-23$ & $3.33 \mathrm{E}-34$ & $0.00 \mathrm{E}+00$ & $0.00 \mathrm{E}+00$ & $0.00 \mathrm{E}+00$ & $0.00 \mathrm{E}+00$ \\
\hline IR191 & $1.95 \mathrm{E}-16$ & $2.09 \mathrm{E}-16$ & $2.22 \mathrm{E}-16$ & $3.09 \mathrm{E}-16$ & $4.29 \mathrm{E}-16$ & $5.06 \mathrm{E}-16$ & $5.11 \mathrm{E}-16$ & $5.11 \mathrm{E}-16$ & $5.11 \mathrm{E}-16$ \\
\hline IR192 & $3.72 \mathrm{E}-17$ & $3.69 \mathrm{E}-17$ & $3.65 \mathrm{E}-17$ & $3.39 \mathrm{E}-17$ & $2.81 \mathrm{E}-17$ & $1.60 \mathrm{E}-17$ & $1.10 \mathrm{E}-17$ & $6.90 \mathrm{E}-18$ & $1.28 \mathrm{E}-18$ \\
\hline PT192 & $5.22 \mathrm{E}-18$ & $5.55 \mathrm{E}-18$ & $5.87 \mathrm{E}-18$ & $8.38 \mathrm{E}-18$ & $1.39 \mathrm{E}-17$ & $2.54 \mathrm{E}-17$ & $3.02 \mathrm{E}-17$ & $3.41 \mathrm{E}-17$ & $3.94 \mathrm{E}-17$ \\
\hline SUMTOT & $7.33 \mathrm{E}+02$ & $7.33 \mathrm{E}+02$ & $7.33 \mathrm{E}+02$ & $7.33 \mathrm{E}+02$ & $7.33 \mathrm{E}+02$ & $7.33 \mathrm{E}+02$ & $7.33 \mathrm{E}+02$ & $7.33 \mathrm{E}+02$ & $7.33 \mathrm{E}+02$ \\
\hline 0TOTAL & $7.33 \mathrm{E}+02$ & $7.33 \mathrm{E}+02$ & $7.33 \mathrm{E}+02$ & $7.33 \mathrm{E}+02$ & $7.33 \mathrm{E}+02$ & $7.33 \mathrm{E}+02$ & $7.33 \mathrm{E}+02$ & $7.33 \mathrm{E}+02$ & $7.33 \mathrm{E}+02$ \\
\hline
\end{tabular}


BSU-8242 3 DPA As-Run Physics Analysis

Table 23. Source Terms for 'A7 400C 3 DPA Y' Capsule.

\begin{tabular}{|c|c|c|c|c|c|c|c|c|c|}
\hline \multicolumn{10}{|c|}{ ACTIVATION PRODUCTS (grams) } \\
\hline ISOTOPE & EOC & 1 Day & 2 Days & 10 Days & 30 Days & 90 Days & 130 Days & 180 Days & 360 Days \\
\hline H 1 & $7.64 \mathrm{E}-04$ & $7.64 \mathrm{E}-04$ & $7.64 \mathrm{E}-04$ & $7.64 \mathrm{E}-04$ & $7.64 \mathrm{E}-04$ & 7.64E-04 & $7.64 \mathrm{E}-04$ & $7.64 \mathrm{E}-04$ & 7.64E-04 \\
\hline H 2 & $3.86 \mathrm{E}-07$ & $3.86 \mathrm{E}-07$ & $3.86 \mathrm{E}-07$ & $3.86 \mathrm{E}-07$ & $3.86 \mathrm{E}-07$ & $3.86 \mathrm{E}-07$ & $3.86 \mathrm{E}-07$ & $3.86 \mathrm{E}-07$ & $3.86 \mathrm{E}-07$ \\
\hline H 3 & 1.07E-08 & 1.07E-08 & $1.07 \mathrm{E}-08$ & 1.07E-08 & $1.06 \mathrm{E}-08$ & $1.05 \mathrm{E}-08$ & $1.05 \mathrm{E}-08$ & 1.04E-08 & $1.01 \mathrm{E}-08$ \\
\hline HE 3 & $4.60 \mathrm{E}-11$ & $4.76 \mathrm{E}-11$ & $4.92 \mathrm{E}-11$ & $6.24 \mathrm{E}-11$ & $9.51 \mathrm{E}-11$ & $1.93 \mathrm{E}-10$ & $2.57 \mathrm{E}-10$ & $3.38 \mathrm{E}-10$ & $6.21 \mathrm{E}-10$ \\
\hline HE 4 & $1.27 \mathrm{E}-02$ & $1.27 \mathrm{E}-02$ & $1.27 \mathrm{E}-02$ & $1.27 \mathrm{E}-02$ & $1.27 \mathrm{E}-02$ & $1.27 \mathrm{E}-02$ & $1.27 \mathrm{E}-02$ & $1.27 \mathrm{E}-02$ & $1.27 \mathrm{E}-02$ \\
\hline HE 6 & $6.90 \mathrm{E}-17$ & $0.00 \mathrm{E}+00$ & $0.00 \mathrm{E}+00$ & $0.00 \mathrm{E}+00$ & $0.00 \mathrm{E}+00$ & $0.00 \mathrm{E}+00$ & $0.00 \mathrm{E}+00$ & $0.00 \mathrm{E}+00$ & $0.00 \mathrm{E}+00$ \\
\hline LI 6 & $2.10 \mathrm{E}-10$ & $2.10 \mathrm{E}-10$ & $2.10 \mathrm{E}-10$ & $2.10 \mathrm{E}-10$ & $2.10 \mathrm{E}-10$ & $2.10 \mathrm{E}-10$ & $2.10 \mathrm{E}-10$ & & \\
\hline LI 7 & $2.27 \mathrm{E}-13$ & $2.27 \mathrm{E}-13$ & $2.27 \mathrm{E}-13$ & $2.27 \mathrm{E}-13$ & $2.27 \mathrm{E}-13$ & $2.27 \mathrm{E}-13$ & 2.27 & $2.27 \mathrm{E}-13$ & $2.27 \mathrm{E}-13$ \\
\hline BE 9 & $2.49 \mathrm{E}-06$ & $2.49 \mathrm{E}-06$ & $2.49 \mathrm{E}-06$ & $2.49 \mathrm{E}-06$ & $2.49 \mathrm{E}-06$ & $2.49 \mathrm{E}-06$ & $2.49 \mathrm{E}-06$ & $2.49 \mathrm{E}-06$ & $2.49 \mathrm{E}-06$ \\
\hline $\mathrm{BE} 10$ & E-08 & $6.37 \mathrm{E}-08$ & $6.37 \mathrm{E}-08$ & $6.37 \mathrm{E}-08$ & $6.37 \mathrm{E}-08$ & $6.37 \mathrm{E}-08$ & $6.37 \mathrm{E}-08$ & $6.37 \mathrm{E}-08$ & $6.37 \mathrm{E}-08$ \\
\hline B 10 & $1.25 \mathrm{E}-13$ & $1.25 \mathrm{E}-13$ & $1.25 \mathrm{E}-13$ & $1.25 \mathrm{E}-13$ & $1.27 \mathrm{E}-13$ & $1.32 \mathrm{E}-13$ & $1.35 \mathrm{E}-13$ & $1.38 \mathrm{E}-13$ & $1.52 \mathrm{E}-13$ \\
\hline B 11 & $8.38 \mathrm{E}-05$ & 8.38E-05 & $8.38 \mathrm{E}-05$ & 8.38E-05 & $8.38 \mathrm{E}-05$ & $8.38 \mathrm{E}-05$ & $8.38 \mathrm{E}-05$ & 8.38E-05 & $8.38 \mathrm{E}-05$ \\
\hline C 12 & $5.88 \mathrm{E}-01$ & $5.88 \mathrm{E}-01$ & $5.88 \mathrm{E}-01$ & $5.88 \mathrm{E}-01$ & $5.88 \mathrm{E}-01$ & $5.88 \mathrm{E}-01$ & $5.88 \mathrm{E}-01$ & & $5.88 \mathrm{E}-01$ \\
\hline C 13 & & & & & & & & & \\
\hline C 14 & -04 & 7.3 & 7.3 & 7.3 & 7.36 & E-04 & 7.3 & 7.3 & $7.35 \mathrm{E}-04$ \\
\hline $\mathrm{N} 14$ & $2.79 \mathrm{E}-01$ & $2.79 \mathrm{E}-01$ & $2.79 \mathrm{E}-01$ & $2.79 \mathrm{E}-01$ & $2.79 \mathrm{E}-01$ & $2.79 \mathrm{E}-01$ & $2.79 \mathrm{E}-01$ & $2.79 \mathrm{E}-01$ & $2.79 \mathrm{E}-01$ \\
\hline $\mathrm{N} 15$ & $1.13 \mathrm{E}-03$ & 1.13E-03 & $1.13 \mathrm{E}-03$ & $1.13 \mathrm{E}-03$ & $1.13 \mathrm{E}-03$ & $1.13 \mathrm{E}-03$ & $1.13 \mathrm{E}-03$ & 1.13E-03 & $1.13 \mathrm{E}-03$ \\
\hline N 16 & $2.55 \mathrm{E}-17$ & $0.00 \mathrm{E}+00$ & $0.00 \mathrm{E}+00$ & $0.00 \mathrm{E}+00$ & $0.00 \mathrm{E}+00$ & $0.00 \mathrm{E}+00$ & $0.00 \mathrm{E}+00$ & $0.00 \mathrm{E}+00$ & $0.00 \mathrm{E}+00$ \\
\hline $\mathrm{O} 16$ & $3.82 \mathrm{E}$ & & & $3.82 \mathrm{E}-11$ & & & & & $3.82 \mathrm{E}-11$ \\
\hline O 17 & $4.16 \mathrm{E}-17$ & $4.16 \mathrm{E}-17$ & $4.16 \mathrm{E}-17$ & $4.16 \mathrm{E}-17$ & $4.16 \mathrm{E}-17$ & $4.16 \mathrm{E}-17$ & $4.16 \mathrm{E}-17$ & $4.16 \mathrm{E}-17$ & $4.16 \mathrm{E}-17$ \\
\hline O 18 & $3.77 \mathrm{E}-09$ & $3.77 \mathrm{E}-09$ & $3.77 \mathrm{E}-09$ & $3.77 \mathrm{E}-09$ & $3.77 \mathrm{E}-09$ & $3.77 \mathrm{E}-09$ & $3.77 \mathrm{E}-09$ & $3.77 \mathrm{E}-09$ & $3.77 \mathrm{E}-09$ \\
\hline F 19 & $6.33 \mathrm{E}-16$ & $6.33 \mathrm{E}-16$ & $6.33 \mathrm{E}-16$ & $6.33 \mathrm{E}-16$ & $6.33 \mathrm{E}-16$ & $6.33 \mathrm{E}-16$ & $6.33 \mathrm{E}-16$ & $6.33 \mathrm{E}-16$ & $6.33 \mathrm{E}-16$ \\
\hline NE 20 & $1.46 \mathrm{E}-12$ & $1.46 \mathrm{E}-12$ & $1.46 \mathrm{E}-12$ & $1.46 \mathrm{E}-12$ & $1.46 \mathrm{E}-12$ & $1.46 \mathrm{E}-12$ & $1.46 \mathrm{E}-12$ & $1.46 \mathrm{E}-12$ & $1.46 \mathrm{E}-12$ \\
\hline NE 21 & $4.39 \mathrm{E}-06$ & 4.39E-06 & 4.39E-06 & 4.39E-06 & 4.39E-06 & $4.39 \mathrm{E}-06$ & $4.39 \mathrm{E}-06$ & 4.39E-06 & $4.39 \mathrm{E}-06$ \\
\hline NE 22 & $6.60 \mathrm{E}-06$ & 6.60E-06 & $6.60 \mathrm{E}-06$ & 6.60E-06 & $6.60 \mathrm{E}-06$ & 6.60E-06 & $6.60 \mathrm{E}-06$ & 6.60E-06 & $6.60 \mathrm{E}-06$ \\
\hline
\end{tabular}


BSU-8242 3 DPA As-Run Physics Analysis

\begin{tabular}{|c|c|c|c|c|c|c|c|c|c|}
\hline \multicolumn{10}{|c|}{ ACTIVATION PRODUCTS (grams) } \\
\hline ISOTOPE & EOC & 1 Day & 2 Days & 10 Days & 30 Days & 90 Days & 130 Days & 180 Days & 360 Days \\
\hline NE 23 & $4.62 \mathrm{E}-12$ & $0.00 \mathrm{E}+00$ & $0.00 \mathrm{E}+00$ & $0.00 \mathrm{E}+00$ & $0.00 \mathrm{E}+00$ & $0.00 \mathrm{E}+00$ & $0.00 \mathrm{E}+00$ & $0.00 \mathrm{E}+00$ & $0.00 \mathrm{E}+00$ \\
\hline NA 23 & $34 \mathrm{E}-06$ & $1.34 \mathrm{E}-06$ & $34 \mathrm{E}-06$ & $34 \mathrm{E}-06$ & $34 \mathrm{E}-06$ & $34 \mathrm{E}-06$ & $1.34 \mathrm{E}-06$ & $1.34 \mathrm{E}-06$ & $1.34 \mathrm{E}-06$ \\
\hline NA 24 & $72 \mathrm{E}-06$ & $5.67 \mathrm{E}-07$ & $1.87 \mathrm{E}-07$ & $2.62 \mathrm{E}-11$ & $6.10 \mathrm{E}-21$ & $0.00 \mathrm{E}+00$ & $0.00 \mathrm{E}+00$ & $0.00 \mathrm{E}+00$ & $0.00 \mathrm{E}+00$ \\
\hline NA 25 & 4.86E-12 & $0.00 \mathrm{E}+00$ & $0.00 \mathrm{E}+00$ & $0.00 \mathrm{E}+00$ & $0.00 \mathrm{E}+00$ & $0.00 \mathrm{E}+00$ & $0.00 \mathrm{E}+00$ & $0.00 \mathrm{E}+00$ & $0.00 \mathrm{E}+00$ \\
\hline MG 24 & $7.52 \mathrm{E}-01$ & $7.52 \mathrm{E}-01$ & 7.52E-01 & 7.52E-01 & 7.52E-01 & 7.52E-01 & 7.52E-01 & 7.52E-01 & 7.52E-01 \\
\hline MG 25 & $9.91 \mathrm{E}-02$ & $9.91 \mathrm{E}-02$ & $9.91 \mathrm{E}-02$ & & & 02 & $9.91 \mathrm{E}-02$ & $9.91 \mathrm{E}-02$ & 02 \\
\hline MG 26 & 114501 & 1.14E-01 & $1.14 \mathrm{E}-01$ & $1.14 \mathrm{E}-01$ & $1.14 \mathrm{E}-01$ & $14 \mathrm{E}-01$ & 1.14E-01 & $1.14 \mathrm{E}-01$ & $1.14 \mathrm{E}-01$ \\
\hline MG 27 & $\mathrm{E}-08$ & $0.00 \mathrm{E}+00$ & $0.00 \mathrm{E}+00$ & $0.00 \mathrm{E}+00$ & $0.00 \mathrm{E}+00$ & $0.00 \mathrm{E}+00$ & $0.00 \mathrm{E}+00$ & $0.00 \mathrm{E}+00$ & $0.00 \mathrm{E}+00$ \\
\hline MG 28 & $3.72 \mathrm{E}-14$ & $1.68 \mathrm{E}-14$ & $7.57 \mathrm{E}-15$ & $1.30 \mathrm{E}-17$ & $1.61 \mathrm{E}-24$ & $0.00 \mathrm{E}+00$ & $0.00 \mathrm{E}+00$ & $0.00 \mathrm{E}+00$ & $0.00 \mathrm{E}+00$ \\
\hline AL 27 & $1.32 \mathrm{E}+02$ & $1.32 \mathrm{E}+02$ & $1.32 \mathrm{E}+02$ & $1.32 \mathrm{E}+02$ & $1.32 \mathrm{E}+02$ & $1.32 \mathrm{E}+02$ & $1.32 \mathrm{E}+02$ & $1.32 \mathrm{E}+02$ & $1.32 \mathrm{E}+02$ \\
\hline AL 28 & & 3.00E-17 & $1.35 \mathrm{E}-17$ & & & $0.00 \mathrm{E}+00$ & $0.00 \mathrm{E}+00$ & $0.00 \mathrm{E}+00$ & $0.00 \mathrm{E}+00$ \\
\hline & & $0.00 \mathrm{E}+00$ & $E+00$ & $0 \mathrm{E}+00$ & $0.00 \mathrm{E}+00$ & $0 \mathrm{E}+00$ & $0.00 \mathrm{E}+00$ & $0.00 \mathrm{E}+00$ & $\underline{0 \mathrm{E}+00}$ \\
\hline AL 30 & & & & 00 & +00 & $0.00 \mathrm{E}+00$ & $0.00 \mathrm{E}+00$ & $0.00 \mathrm{E}+00$ & $0.00 \mathrm{E}+00$ \\
\hline SI 28 & $1.91 \mathrm{E}+00$ & $1.91 \mathrm{E}+00$ & $1.91 \mathrm{E}+00$ & $1.91 \mathrm{E}+00$ & $1.91 \mathrm{E}+00$ & $1.91 \mathrm{E}+00$ & $1.91 \mathrm{E}+00$ & $1.91 \mathrm{E}+00$ & $1.91 \mathrm{E}+00$ \\
\hline SI 29 & $9.84 \mathrm{E}-02$ & $9.84 \mathrm{E}-02$ & $9.84 \mathrm{E}-02$ & $9.84 \mathrm{E}-02$ & $9.84 \mathrm{E}-02$ & $9.84 \mathrm{E}-02$ & $9.84 \mathrm{E}-02$ & $9.84 \mathrm{E}-02$ & $9.84 \mathrm{E}-02$ \\
\hline SI 30 & $6.73 \mathrm{E}-02$ & $6.73 \mathrm{E}-02$ & $6.73 \mathrm{E}-02$ & $6.73 \mathrm{E}-02$ & $6.73 \mathrm{E}-02$ & $6.73 \mathrm{E}-02$ & $6.73 \mathrm{E}-02$ & $6.73 \mathrm{E}-02$ & $6.73 \mathrm{E}-02$ \\
\hline SI 31 & & & & & & & & & \\
\hline SI 32 & 11 & -11 & 11 & -11 & -11 & 1.07E-11 & $1.07 \mathrm{E}-11$ & 1.07E-11 & $1.07 \mathrm{E}-11$ \\
\hline P 31 & $5.70 \mathrm{E}-02$ & $5.70 \mathrm{E}-02$ & $5.70 \mathrm{E}-02$ & $5.70 \mathrm{E}-02$ & $5.70 \mathrm{E}-02$ & $5.70 \mathrm{E}-02$ & $5.70 \mathrm{E}-02$ & $5.70 \mathrm{E}-02$ & $5.70 \mathrm{E}-02$ \\
\hline P 32 & $2.62 \mathrm{E}-06$ & $2.50 \mathrm{E}-06$ & $2.38 \mathrm{E}-06$ & 1.61E-06 & $6.12 \mathrm{E}-07$ & $3.34 \mathrm{E}-08$ & $4.80 \mathrm{E}-09$ & $4.26 \mathrm{E}-10$ & $6.98 \mathrm{E}-14$ \\
\hline P 33 & $1.50 \mathrm{E}-10$ & $1.46 \mathrm{E}-10$ & $1.42 \mathrm{E}-10$ & $1.14 \mathrm{E}-10$ & $6.54 \mathrm{E}-11$ & $1.24 \mathrm{E}-11$ & $4.08 \mathrm{E}-12$ & $1.02 \mathrm{E}-12$ & $6.95 \mathrm{E}-15$ \\
\hline P 34 & & $0.00 \mathrm{E}+00$ & $0.00 \mathrm{E}+00$ & $0.00 \mathrm{E}+00$ & $0.00 \mathrm{E}+00$ & $0.00 \mathrm{E}+00$ & $0.00 \mathrm{E}+00$ & $0.00 \mathrm{E}+00$ & $0.00 \mathrm{E}+00$ \\
\hline S 32 & 3. & $3.67 \mathrm{E}-02$ & & & 3.671 & $3.67 \mathrm{E}-02$ & $3.67 \mathrm{E}-02$ & $3.67 \mathrm{E}-02$ & $3.67 \mathrm{E}-02$ \\
\hline S 33 & $3.25 \mathrm{E}-04$ & $3.25 \mathrm{E}-04$ & $3.25 \mathrm{E}-04$ & $3.25 \mathrm{E}-04$ & $3.25 \mathrm{E}-04$ & $3.25 \mathrm{E}-04$ & $3.25 \mathrm{E}-04$ & $3.25 \mathrm{E}-04$ & $3.25 \mathrm{E}-04$ \\
\hline S 34 & $1.73 \mathrm{E}-03$ & $1.73 \mathrm{E}-03$ & $1.73 \mathrm{E}-03$ & $1.73 \mathrm{E}-03$ & $1.73 \mathrm{E}-03$ & $1.73 \mathrm{E}-03$ & $1.73 \mathrm{E}-03$ & $1.73 \mathrm{E}-03$ & $1.73 \mathrm{E}-03$ \\
\hline S 35 & 2.36E-07 & 2.34E-07 & 2.32E-07 & $2.18 \mathrm{E}-07$ & $1.86 \mathrm{E}-07$ & 1.16E-07 & 8.47E-08 & 5.71E-08 & $1.38 \mathrm{E}-08$ \\
\hline
\end{tabular}


BSU-8242 3 DPA As-Run Physics Analysis

\begin{tabular}{|c|c|c|c|c|c|c|c|c|c|}
\hline \multicolumn{10}{|c|}{ ACTIVATION PRODUCTS (grams) } \\
\hline ISOTOPE & EOC & 1 Day & 2 Days & 10 Days & 30 Days & 90 Days & 130 Days & 180 Days & 360 Days \\
\hline S 36 & $7.38 \mathrm{E}-06$ & $7.38 \mathrm{E}-06$ & $7.38 \mathrm{E}-06$ & $7.38 \mathrm{E}-06$ & $7.38 \mathrm{E}-06$ & $7.38 \mathrm{E}-06$ & 7.38E-06 & 7.38E-06 & 7.38E-06 \\
\hline S 37 & $4.27 \mathrm{E}-14$ & $0.00 \mathrm{E}+00$ & $0.00 \mathrm{E}+00$ & $0.00 \mathrm{E}+00$ & $0.00 \mathrm{E}+00$ & $0.00 \mathrm{E}+00$ & $0.00 \mathrm{E}+00$ & $0.00 \mathrm{E}+00$ & $0.00 \mathrm{E}+00$ \\
\hline CL 35 & $3.25 \mathrm{E}-07$ & $3.27 \mathrm{E}-07$ & $3.29 \mathrm{E}-07$ & $3.43 \mathrm{E}-07$ & $3.75 \mathrm{E}-07$ & $4.45 \mathrm{E}-07$ & $4.76 \mathrm{E}-07$ & $5.04 \mathrm{E}-07$ & $5.47 \mathrm{E}-07$ \\
\hline CL 36 & $9.07 \mathrm{E}-09$ & $9.07 \mathrm{E}-09$ & $9.07 \mathrm{E}-09$ & $9.07 \mathrm{E}-09$ & $9.07 \mathrm{E}-09$ & $9.07 \mathrm{E}-09$ & $9.07 \mathrm{E}-09$ & 9.07E-09 & 9.07E-09 \\
\hline CL 37 & $1.56 \mathrm{E}-09$ & $1.56 \mathrm{E}-09$ & $1.56 \mathrm{E}-09$ & $1.56 \mathrm{E}-09$ & $1.56 \mathrm{E}-09$ & $1.56 \mathrm{E}-09$ & $1.56 \mathrm{E}-09$ & $1.56 \mathrm{E}-09$ & $1.56 \mathrm{E}-09$ \\
\hline CL 38 & $2.03 \mathrm{E}-16$ & $4.57 \mathrm{E}-28$ & $1.03 \mathrm{E}-39$ & $0.00 \mathrm{E}+00$ & $0.00 \mathrm{E}+00$ & $0.00 \mathrm{E}+00$ & $0.00 \mathrm{E}+00$ & $0.00 \mathrm{E}+00$ & $0.00 \mathrm{E}+00$ \\
\hline AR 36 & $4.11 \mathrm{E}-15$ & $4.17 \mathrm{E}-15$ & $4.23 \mathrm{E}-15$ & $4.68 \mathrm{E}-15$ & $5.80 \mathrm{E}-15$ & $9.16 \mathrm{E}-15$ & $1.14 \mathrm{E}-14$ & $1.42 \mathrm{E}-14$ & $2.43 \mathrm{E}-14$ \\
\hline AR 38 & $4.84 \mathrm{E}-13$ & $4.84 \mathrm{E}-13$ & $4.84 \mathrm{E}-13$ & 4.84E-13 & 4.84E-13 & 4.84E-13 & 4.84E-13 & 4.84E-13 & $4.84 \mathrm{E}-13$ \\
\hline AR 39 & $1.48 \mathrm{E}-16$ & $1.48 \mathrm{E}-16$ & $1.48 \mathrm{E}-16$ & $1.48 \mathrm{E}-16$ & $1.48 \mathrm{E}-16$ & $1.48 \mathrm{E}-16$ & $1.48 \mathrm{E}-16$ & $1.47 \mathrm{E}-16$ & $1.47 \mathrm{E}-16$ \\
\hline AR 40 & $3.14 \mathrm{E}-17$ & $3.14 \mathrm{E}-17$ & $3.14 \mathrm{E}-17$ & $3.14 \mathrm{E}-17$ & $3.14 \mathrm{E}-17$ & $3.14 \mathrm{E}-17$ & $3.14 \mathrm{E}-17$ & $3.14 \mathrm{E}-17$ & $3.14 \mathrm{E}-17$ \\
\hline CA 44 & $1.57 \mathrm{E}-10$ & $1.57 \mathrm{E}-10$ & $1.57 \mathrm{E}-10$ & $1.57 \mathrm{E}-10$ & $1.57 \mathrm{E}-10$ & $1.57 \mathrm{E}-10$ & $1.57 \mathrm{E}-10$ & $1.57 \mathrm{E}-10$ & $1.57 \mathrm{E}-10$ \\
\hline CA 45 & $5.91 \mathrm{E}-14$ & $5.89 \mathrm{E}-14$ & $5.86 \mathrm{E}-14$ & $5.67 \mathrm{E}-14$ & $5.21 \mathrm{E}-14$ & $4.03 \mathrm{E}-14$ & $3.40 \mathrm{E}-14$ & $2.75 \mathrm{E}-14$ & $1.28 \mathrm{E}-14$ \\
\hline CA 46 & $6.29 \mathrm{E}-11$ & $6.29 \mathrm{E}-11$ & $6.29 \mathrm{E}-11$ & $6.29 \mathrm{E}-11$ & $6.29 \mathrm{E}-11$ & $6.29 \mathrm{E}-11$ & $6.29 \mathrm{E}-11$ & $6.29 \mathrm{E}-11$ & $6.29 \mathrm{E}-11$ \\
\hline CA 47 & $1.13 \mathrm{E}-14$ & $9.73 \mathrm{E}-15$ & $8.35 \mathrm{E}-15$ & $2.46 \mathrm{E}-15$ & $1.16 \mathrm{E}-16$ & $1.21 \mathrm{E}-20$ & $2.51 \mathrm{E}-23$ & $1.20 \mathrm{E}-26$ & $1.36 \mathrm{E}-38$ \\
\hline $\mathrm{SC} 45$ & $1.48 \mathrm{E}-14$ & $1.50 \mathrm{E}-14$ & $1.53 \mathrm{E}-14$ & $1.72 \mathrm{E}-14$ & $2.18 \mathrm{E}-14$ & $3.36 \mathrm{E}-14$ & $3.99 \mathrm{E}-14$ & $4.64 \mathrm{E}-14$ & $6.11 \mathrm{E}-14$ \\
\hline $\mathrm{SC} 46$ & $1.02 \mathrm{E}-16$ & $1.01 \mathrm{E}-16$ & $1.01 \mathrm{E}-16$ & $9.41 \mathrm{E}-17$ & $7.98 \mathrm{E}-17$ & $4.86 \mathrm{E}-17$ & $3.49 \mathrm{E}-17$ & $2.31 \mathrm{E}-17$ & $5.20 \mathrm{E}-18$ \\
\hline $\mathrm{SC} 47$ & $3.58 \mathrm{E}-11$ & $2.91 \mathrm{E}-11$ & $2.37 \mathrm{E}-11$ & $4.53 \mathrm{E}-12$ & $7.25 \mathrm{E}-14$ & $3.28 \mathrm{E}-19$ & $1.59 \mathrm{E}-22$ & $3.69 \mathrm{E}-26$ & $2.50 \mathrm{E}-42$ \\
\hline $\mathrm{SC} 48$ & $4.53 \mathrm{E}-11$ & $3.10 \mathrm{E}-11$ & $2.12 \mathrm{E}-11$ & $1.02 \mathrm{E}-12$ & $5.11 \mathrm{E}-16$ & $6.49 \mathrm{E}-26$ & $1.64 \mathrm{E}-32$ & $9.32 \mathrm{E}-41$ & $0.00 \mathrm{E}+00$ \\
\hline SC 49 & $1.22 \mathrm{E}-13$ & $3.52 \mathrm{E}-21$ & $1.02 \mathrm{E}-28$ & $0.00 \mathrm{E}+00$ & $0.00 \mathrm{E}+00$ & $0.00 \mathrm{E}+00$ & $0.00 \mathrm{E}+00$ & $0.00 \mathrm{E}+00$ & $0.00 \mathrm{E}+00$ \\
\hline SC 50 & $3.16 \mathrm{E}-16$ & $0.00 \mathrm{E}+00$ & $0.00 \mathrm{E}+00$ & $0.00 \mathrm{E}+00$ & $0.00 \mathrm{E}+00$ & $0.00 \mathrm{E}+00$ & $0.00 \mathrm{E}+00$ & $0.00 \mathrm{E}+00$ & $0.00 \mathrm{E}+00$ \\
\hline TI 46 & $3.70 \mathrm{E}-17$ & $3.78 \mathrm{E}-17$ & $3.87 \mathrm{E}-17$ & $4.51 \mathrm{E}-17$ & $5.95 \mathrm{E}-17$ & $9.07 \mathrm{E}-17$ & $1.04 \mathrm{E}-16$ & $1.16 \mathrm{E}-16$ & $1.34 \mathrm{E}-16$ \\
\hline TI 47 & $1.36 \mathrm{E}-05$ & $1.36 \mathrm{E}-05$ & $1.36 \mathrm{E}-05$ & $1.36 \mathrm{E}-05$ & $1.36 \mathrm{E}-05$ & $1.36 \mathrm{E}-05$ & $1.36 \mathrm{E}-05$ & $1.36 \mathrm{E}-05$ & $1.36 \mathrm{E}-05$ \\
\hline TI 48 & $2.08 \mathrm{E}-08$ & $2.08 \mathrm{E}-08$ & $2.08 \mathrm{E}-08$ & $2.08 \mathrm{E}-08$ & $2.08 \mathrm{E}-08$ & $2.08 \mathrm{E}-08$ & $2.08 \mathrm{E}-08$ & $2.08 \mathrm{E}-08$ & $2.08 \mathrm{E}-08$ \\
\hline TI 49 & $3.72 \mathrm{E}-05$ & $3.72 \mathrm{E}-05$ & $3.72 \mathrm{E}-05$ & $3.72 \mathrm{E}-05$ & $3.72 \mathrm{E}-05$ & $3.72 \mathrm{E}-05$ & $3.72 \mathrm{E}-05$ & $3.72 \mathrm{E}-05$ & $3.72 \mathrm{E}-05$ \\
\hline TI 50 & $1.50 \mathrm{E}-04$ & $1.50 \mathrm{E}-04$ & $1.50 \mathrm{E}-04$ & $1.50 \mathrm{E}-04$ & $1.50 \mathrm{E}-04$ & $1.50 \mathrm{E}-04$ & $1.50 \mathrm{E}-04$ & $1.50 \mathrm{E}-04$ & $1.50 \mathrm{E}-04$ \\
\hline TI 51 & $2.45 \mathrm{E}-11$ & $0.00 \mathrm{E}+00$ & $0.00 \mathrm{E}+00$ & $0.00 \mathrm{E}+00$ & $0.00 \mathrm{E}+00$ & $0.00 \mathrm{E}+00$ & $0.00 \mathrm{E}+00$ & $0.00 \mathrm{E}+00$ & $0.00 \mathrm{E}+00$ \\
\hline
\end{tabular}


BSU-8242 3 DPA As-Run Physics Analysis

\begin{tabular}{|c|c|c|c|c|c|c|c|c|c|}
\hline \multicolumn{10}{|c|}{ ACTIVATION PRODUCTS (grams) } \\
\hline ISOTOPE & EOC & 1 Day & 2 Days & 10 Days & 30 Days & 90 Days & 130 Days & 180 Days & 360 Days \\
\hline V 50 & $8.05 \mathrm{E}-04$ & $8.05 \mathrm{E}-04$ & $8.05 \mathrm{E}-04$ & $8.05 \mathrm{E}-04$ & $8.05 \mathrm{E}-04$ & $8.05 \mathrm{E}-04$ & $8.05 \mathrm{E}-04$ & $8.05 \mathrm{E}-04$ & $8.05 \mathrm{E}-04$ \\
\hline V 51 & $2.09 \mathrm{E}-01$ & $2.10 \mathrm{E}-01$ & $2.10 \mathrm{E}-01$ & $2.14 \mathrm{E}-01$ & $2.20 \mathrm{E}-01$ & $2.28 \mathrm{E}-01$ & $2.29 \mathrm{E}-01$ & $2.30 \mathrm{E}-01$ & $2.30 \mathrm{E}-01$ \\
\hline $\mathrm{V} 52$ & $4.43 \mathrm{E}-08$ & $0.00 \mathrm{E}+00$ & $0.00 \mathrm{E}+00$ & $0.00 \mathrm{E}+00$ & $0.00 \mathrm{E}+00$ & $0.00 \mathrm{E}+00$ & $0.00 \mathrm{E}+00$ & $0.00 \mathrm{E}+00$ & $0.00 \mathrm{E}+00$ \\
\hline V 53 & $4.73 \mathrm{E}-11$ & $0.00 \mathrm{E}+00$ & $0.00 \mathrm{E}+00$ & $0.00 \mathrm{E}+00$ & $0.00 \mathrm{E}+00$ & $0.00 \mathrm{E}+00$ & $0.00 \mathrm{E}+00$ & $0.00 \mathrm{E}+00$ & $0.00 \mathrm{E}+00$ \\
\hline $\mathrm{V} 54$ & $2.97 \mathrm{E}-13$ & $0.00 \mathrm{E}+00$ & $0.00 \mathrm{E}+00$ & $0.00 \mathrm{E}+00$ & $0.00 \mathrm{E}+00$ & $0.00 \mathrm{E}+00$ & $0.00 \mathrm{E}+00$ & $0.00 \mathrm{E}+00$ & $0.00 \mathrm{E}+00$ \\
\hline CR 50 & $4.97 E+00$ & $4.97 \mathrm{E}+00$ & $4.97 \mathrm{E}+00$ & $4.97 \mathrm{E}+00$ & $4.97 \mathrm{E}+00$ & $4.97 \mathrm{E}+00$ & $4.97 \mathrm{E}+00$ & $4.97 \mathrm{E}+00$ & $4.97 \mathrm{E}+00$ \\
\hline CR 51 & $2.07 \mathrm{E}-02$ & $2.02 \mathrm{E}-02$ & $1.97 \mathrm{E}-02$ & $1.61 \mathrm{E}-02$ & $9.78 \mathrm{E}-03$ & $2.18 \mathrm{E}-03$ & 8.02E-04 & $2.30 \mathrm{E}-04$ & $2.54 \mathrm{E}-06$ \\
\hline CR 52 & $1.01 \mathrm{E}+02$ & $1.01 \mathrm{E}+02$ & $1.01 \mathrm{E}+02$ & $1.01 \mathrm{E}+02$ & $1.01 \mathrm{E}+02$ & $1.01 \mathrm{E}+02$ & $1.01 \mathrm{E}+02$ & $1.01 \mathrm{E}+02$ & $1.01 \mathrm{E}+02$ \\
\hline CR 53 & $1.20 \mathrm{E}+01$ & $1.20 \mathrm{E}+01$ & $1.20 \mathrm{E}+01$ & $1.20 \mathrm{E}+01$ & $1.20 \mathrm{E}+01$ & $1.20 \mathrm{E}+01$ & $1.20 \mathrm{E}+01$ & $1.20 \mathrm{E}+01$ & $1.20 \mathrm{E}+01$ \\
\hline CR 54 & $3.27 \mathrm{E}+00$ & $3.27 \mathrm{E}+00$ & $3.27 \mathrm{E}+00$ & $3.27 \mathrm{E}+00$ & $3.27 \mathrm{E}+00$ & $3.28 \mathrm{E}+00$ & $3.28 \mathrm{E}+00$ & $3.28 \mathrm{E}+00$ & $3.28 \mathrm{E}+00$ \\
\hline CR 55 & $3.26 \mathrm{E}-08$ & $0.00 \mathrm{E}+00$ & $0.00 \mathrm{E}+00$ & $0.00 \mathrm{E}+00$ & $0.00 \mathrm{E}+00$ & $0.00 \mathrm{E}+00$ & $0.00 \mathrm{E}+00$ & $0.00 \mathrm{E}+00$ & $0.00 \mathrm{E}+00$ \\
\hline MN 54 & $5.95 \mathrm{E}-03$ & $5.93 \mathrm{E}-03$ & $5.92 \mathrm{E}-03$ & $5.82 \mathrm{E}-03$ & $5.56 \mathrm{E}-03$ & $4.87 \mathrm{E}-03$ & $4.46 \mathrm{E}-03$ & $3.99 \mathrm{E}-03$ & $2.68 \mathrm{E}-03$ \\
\hline MN 55 & $5.48 \mathrm{E}+00$ & $5.48 \mathrm{E}+00$ & $5.48 \mathrm{E}+00$ & $5.48 \mathrm{E}+00$ & $5.48 \mathrm{E}+00$ & $5.48 \mathrm{E}+00$ & $5.49 \mathrm{E}+00$ & $5.49 \mathrm{E}+00$ & $5.50 \mathrm{E}+00$ \\
\hline MN 56 & $1.16 \mathrm{E}-04$ & $1.83 \mathrm{E}-07$ & $2.88 \mathrm{E}-10$ & $0.00 \mathrm{E}+00$ & $0.00 \mathrm{E}+00$ & $0.00 \mathrm{E}+00$ & $0.00 \mathrm{E}+00$ & $0.00 \mathrm{E}+00$ & $0.00 \mathrm{E}+00$ \\
\hline MN 57 & $2.07 \mathrm{E}-10$ & $0.00 \mathrm{E}+00$ & $0.00 \mathrm{E}+00$ & $0.00 \mathrm{E}+00$ & $0.00 \mathrm{E}+00$ & $0.00 \mathrm{E}+00$ & $0.00 \mathrm{E}+00$ & $0.00 \mathrm{E}+00$ & $0.00 \mathrm{E}+00$ \\
\hline MN 58 & $4.23 \mathrm{E}-13$ & $0.00 \mathrm{E}+00$ & $0.00 \mathrm{E}+00$ & $0.00 \mathrm{E}+00$ & $0.00 \mathrm{E}+00$ & $0.00 \mathrm{E}+00$ & $0.00 \mathrm{E}+00$ & $0.00 \mathrm{E}+00$ & $0.00 \mathrm{E}+00$ \\
\hline FE 54 & $2.84 \mathrm{E}+01$ & $2.84 \mathrm{E}+01$ & $2.84 \mathrm{E}+01$ & $2.84 \mathrm{E}+01$ & $2.84 \mathrm{E}+01$ & $2.84 \mathrm{E}+01$ & $2.84 \mathrm{E}+01$ & $2.84 \mathrm{E}+01$ & $2.84 \mathrm{E}+01$ \\
\hline FE 55 & 7.97E-02 & $7.96 \mathrm{E}-02$ & $7.96 \mathrm{E}-02$ & $7.91 \mathrm{E}-02$ & $7.80 \mathrm{E}-02$ & $7.46 \mathrm{E}-02$ & $7.25 \mathrm{E}-02$ & $6.99 \mathrm{E}-02$ & $6.13 \mathrm{E}-02$ \\
\hline FE 56 & $4.65 \mathrm{E}+02$ & $4.65 \mathrm{E}+02$ & $4.65 \mathrm{E}+02$ & $4.65 \mathrm{E}+02$ & $4.65 \mathrm{E}+02$ & $4.65 \mathrm{E}+02$ & $4.65 \mathrm{E}+02$ & $4.65 \mathrm{E}+02$ & $4.65 \mathrm{E}+02$ \\
\hline FE 57 & $1.30 \mathrm{E}+01$ & $1.30 \mathrm{E}+01$ & $1.30 \mathrm{E}+01$ & $1.30 \mathrm{E}+01$ & $1.30 \mathrm{E}+01$ & $1.30 \mathrm{E}+01$ & $1.30 \mathrm{E}+01$ & $1.30 \mathrm{E}+01$ & $1.30 \mathrm{E}+01$ \\
\hline FE 58 & $1.57 \mathrm{E}+00$ & $1.57 \mathrm{E}+00$ & $1.57 \mathrm{E}+00$ & $1.57 \mathrm{E}+00$ & $1.57 \mathrm{E}+00$ & $1.57 \mathrm{E}+00$ & $1.58 \mathrm{E}+00$ & $1.58 \mathrm{E}+00$ & $1.58 \mathrm{E}+00$ \\
\hline FE 59 & $7.68 \mathrm{E}-04$ & $7.56 \mathrm{E}-04$ & $7.44 \mathrm{E}-04$ & $6.58 \mathrm{E}-04$ & $4.84 \mathrm{E}-04$ & $1.92 \mathrm{E}-04$ & $1.04 \mathrm{E}-04$ & $4.80 \mathrm{E}-05$ & $3.00 \mathrm{E}-06$ \\
\hline CO 58 & $2.21 \mathrm{E}-03$ & $2.19 \mathrm{E}-03$ & $2.17 \mathrm{E}-03$ & $2.00 \mathrm{E}-03$ & $1.65 \mathrm{E}-03$ & $9.16 \mathrm{E}-04$ & $6.19 \mathrm{E}-04$ & $3.79 \mathrm{E}-04$ & $6.51 \mathrm{E}-05$ \\
\hline CO 59 & $9.34 \mathrm{E}-03$ & $9.35 \mathrm{E}-03$ & $9.36 \mathrm{E}-03$ & $9.45 \mathrm{E}-03$ & $9.63 \mathrm{E}-03$ & $9.92 \mathrm{E}-03$ & $1.00 \mathrm{E}-02$ & $1.01 \mathrm{E}-02$ & $1.01 \mathrm{E}-02$ \\
\hline $\mathrm{CO} 60$ & $4.50 \mathrm{E}-04$ & $4.50 \mathrm{E}-04$ & $4.49 \mathrm{E}-04$ & $4.48 \mathrm{E}-04$ & $4.45 \mathrm{E}-04$ & $4.35 \mathrm{E}-04$ & $4.29 \mathrm{E}-04$ & $4.21 \mathrm{E}-04$ & $3.95 \mathrm{E}-04$ \\
\hline $\mathrm{CO} 60 \mathrm{M}$ & $2.10 \mathrm{E}-08$ & $0.00 \mathrm{E}+00$ & $0.00 \mathrm{E}+00$ & $0.00 \mathrm{E}+00$ & $0.00 \mathrm{E}+00$ & $0.00 \mathrm{E}+00$ & $0.00 \mathrm{E}+00$ & $0.00 \mathrm{E}+00$ & $0.00 \mathrm{E}+00$ \\
\hline
\end{tabular}


BSU-8242 3 DPA As-Run Physics Analysis

\begin{tabular}{|c|c|c|c|c|c|c|c|c|c|}
\hline \multicolumn{10}{|c|}{ ACTIVATION PRODUCTS (grams) } \\
\hline ISOTOPE & EOC & 1 Day & 2 Days & 10 Days & 30 Days & 90 Days & 130 Days & 180 Days & 360 Days \\
\hline $\mathrm{CO} 61$ & 4.83E-09 & $2.02 \mathrm{E}-13$ & $8.44 \mathrm{E}-18$ & $0.00 \mathrm{E}+00$ & $0.00 \mathrm{E}+00$ & $0.00 \mathrm{E}+00$ & $0.00 \mathrm{E}+00$ & $0.00 \mathrm{E}+00$ & $0.00 \mathrm{E}+00$ \\
\hline $\mathrm{CO} 62$ & $.09 \mathrm{E}-11$ & $0.00 \mathrm{E}+00$ & $0.00 \mathrm{E}+00$ & $0.00 \mathrm{E}+00$ & $0.00 \mathrm{E}+00$ & $0.00 \mathrm{E}+00$ & $0.00 \mathrm{E}+00$ & $0.00 \mathrm{E}+00$ & $0.00 \mathrm{E}+00$ \\
\hline NI 58 & $6 \mathrm{E}+01$ & $4.86 \mathrm{E}+01$ & $4.86 \mathrm{E}+01$ & $4.86 \mathrm{E}+01$ & $4.86 \mathrm{E}+01$ & $4.86 \mathrm{E}+01$ & $4.86 \mathrm{E}+01$ & $4.86 \mathrm{E}+01$ & $4.86 \mathrm{E}+01$ \\
\hline NI 59 & $3.22 \mathrm{E}-01$ & $3.22 \mathrm{E}-01$ & $3.22 \mathrm{E}-01$ & $3.22 \mathrm{E}-01$ & 3.22E-01 & $3.22 \mathrm{E}-01$ & $3.22 \mathrm{E}-01$ & $3.22 \mathrm{E}-01$ & $3.22 \mathrm{E}-01$ \\
\hline NI 60 & $1.93 \mathrm{E}+01$ & $1.93 \mathrm{E}+01$ & $1.93 \mathrm{E}+01$ & $1.93 \mathrm{E}+01$ & $1.93 \mathrm{E}+01$ & $1.93 \mathrm{E}+01$ & $1.93 \mathrm{E}+01$ & $1.93 \mathrm{E}+01$ & $1.93 \mathrm{E}+01$ \\
\hline NI 61 & $9.24 \mathrm{E}-01$ & $9.24 \mathrm{E}-01$ & $9.24 \mathrm{E}-01$ & $9.24 \mathrm{E}-01$ & $9.24 \mathrm{E}-01$ & $9.24 \mathrm{E}-01$ & $9.24 \mathrm{E}-01$ & $9.24 \mathrm{E}-01$ & $9.24 \mathrm{E}-01$ \\
\hline NI 62 & $2.70 \mathrm{E}+00$ & $2.70 \mathrm{E}+00$ & $2.70 \mathrm{E}+00$ & $2.70 \mathrm{E}+00$ & $2.70 \mathrm{E}+00$ & $2.70 \mathrm{E}+00$ & $2.70 \mathrm{E}+00$ & $2.70 \mathrm{E}+00$ & $2.70 \mathrm{E}+00$ \\
\hline NI & & & & E-02 & & $5.19 \mathrm{E}-02$ & & $\mathrm{E}-02$ & -02 \\
\hline NI 64 & -01 & -01 & E-01 & $7.21 \mathrm{E}-01$ & $7.21 \mathrm{E}-01$ & $7.21 \mathrm{E}-01$ & $7.21 \mathrm{E}-01$ & $7.21 \mathrm{E}-01$ & $7.21 \mathrm{E}-01$ \\
\hline NI 65 & $1.32 \mathrm{E}-06$ & $1.79 \mathrm{E}-09$ & $2.43 \mathrm{E}-12$ & $0.00 \mathrm{E}+00$ & $0.00 \mathrm{E}+00$ & $0.00 \mathrm{E}+00$ & $0.00 \mathrm{E}+00$ & $0.00 \mathrm{E}+00$ & $0.00 \mathrm{E}+00$ \\
\hline NI 66 & 7.91E-10 & $5.83 \mathrm{E}-10$ & $4.30 \mathrm{E}-10$ & $3.76 \mathrm{E}-11$ & $8.50 \mathrm{E}-14$ & $9.81 \mathrm{E}-22$ & $5.01 \mathrm{E}-27$ & $1.22 \mathrm{E}-33$ & $0.00 \mathrm{E}+00$ \\
\hline CU 63 & 3.33E-01 & 3.33E-01 & 3.33E-01 & 3.33E-01 & 3.33E-01 & 3.33E-01 & 3.33E-01 & 3.33E-01 & 3.33E-01 \\
\hline CU 64 & & & & & & $0.00 \mathrm{E}+00$ & $0.00 \mathrm{E}+00$ & $0.00 \mathrm{E}+00$ & $0.00 \mathrm{E}+00$ \\
\hline CU 65 & & & & & & & 01 & 1.5 & -01 \\
\hline CU 66 & 1.52E-08 & $9.10 \mathrm{E}-13$ & $6.71 \mathrm{E}-13$ & $5.86 \mathrm{E}-14$ & 1.331 & $1.53 \mathrm{E}-24$ & 7.82E-30 & 1.90E-36 & $0.00 \mathrm{E}+00$ \\
\hline CU 67 & $5.73 \mathrm{E}-11$ & $4.38 \mathrm{E}-11$ & 3.35E-11 & $3.89 \mathrm{E}-12$ & $1.80 \mathrm{E}-14$ & $1.77 \mathrm{E}-21$ & $3.76 \mathrm{E}-26$ & $5.44 \mathrm{E}-32$ & $0.00 \mathrm{E}+00$ \\
\hline ZN 64 & 8.83E-04 & $8.86 \mathrm{E}-04$ & $8.87 \mathrm{E}-04$ & & 8.87E-04 & 8.87E-04 & 8.87E-04 & 8.87E-04 & $8.87 \mathrm{E}-04$ \\
\hline ZN 65 & $5.05 \mathrm{E}-07$ & & $5.02 \mathrm{E}-07$ & & & 3.91E-07 & 3.49E-07 & 3.03E-07 & $1.81 \mathrm{E}-07$ \\
\hline ZN 66 & BE-04 & 5.3 & 5.33E-04 & 5.3 & 5.33 & 5.33E-04 & 5.33E-04 & 5.33E-04 & $5.33 \mathrm{E}-04$ \\
\hline ZN 67 & $3.48 \mathrm{E}-07$ & $3.48 \mathrm{E}-07$ & $3.48 \mathrm{E}-07$ & $3.48 \mathrm{E}-07$ & $3.48 \mathrm{E}-07$ & $3.48 \mathrm{E}-07$ & $3.48 \mathrm{E}-07$ & $3.48 \mathrm{E}-07$ & $3.48 \mathrm{E}-07$ \\
\hline ZN 68 & $1.80 \mathrm{E}-09$ & $1.80 \mathrm{E}-09$ & $1.80 \mathrm{E}-09$ & 1.80E-09 & 1.80E-09 & $1.80 \mathrm{E}-09$ & $1.80 \mathrm{E}-09$ & 1.80E-09 & $1.80 \mathrm{E}-09$ \\
\hline ZN 69 & $1.41 \mathrm{E}-15$ & 3.0 & $9.08 \mathrm{E}-18$ & $5.73 \mathrm{E}-22$ & $1.81 \mathrm{E}-32$ & $0.00 \mathrm{E}+00$ & $0.00 \mathrm{E}+00$ & $0.00 \mathrm{E}+00$ & $0.00 \mathrm{E}+00$ \\
\hline ZN 69M & 1.37E- 15 & 4.10E-16 & 1.22E-16 & 7.72E-21 & 2.44E-31 & $0.00 \mathrm{E}+00$ & $0.00 \mathrm{E}+00$ & $0.00 \mathrm{E}+00$ & $0.00 \mathrm{E}+00$ \\
\hline GA 69 & $1.12 \mathrm{E}-12$ & $1.12 \mathrm{E}-12$ & $1.12 \mathrm{E}-12$ & $1.12 \mathrm{E}-12$ & $1.12 \mathrm{E}-12$ & $1.12 \mathrm{E}-12$ & $1.12 \mathrm{E}-12$ & $1.12 \mathrm{E}-12$ & $1.12 \mathrm{E}-12$ \\
\hline GE 70 & $1.71 \mathrm{E}-15$ & $1.72 \mathrm{E}-15$ & $1.72 \mathrm{E}-15$ & $1.72 \mathrm{E}-15$ & $1.72 \mathrm{E}-15$ & $1.72 \mathrm{E}-15$ & $1.72 \mathrm{E}-15$ & $1.72 \mathrm{E}-15$ & $1.72 \mathrm{E}-15$ \\
\hline SR 87 & $6.77 \mathrm{E}-17$ & $6.77 \mathrm{E}-17$ & $6.77 \mathrm{E}-17$ & $6.77 \mathrm{E}-17$ & $6.77 \mathrm{E}-17$ & $6.77 \mathrm{E}-17$ & $6.77 \mathrm{E}-17$ & $6.77 \mathrm{E}-17$ & $6.77 \mathrm{E}-17$ \\
\hline
\end{tabular}


BSU-8242 3 DPA As-Run Physics Analysis

\begin{tabular}{|c|c|c|c|c|c|c|c|c|c|}
\hline \multicolumn{10}{|c|}{ ACTIVATION PRODUCTS (grams) } \\
\hline ISOTOPE & EOC & 1 Day & 2 Days & 10 Days & 30 Days & 90 Days & 130 Days & 180 Days & 360 Days \\
\hline SR 88 & $2.66 \mathrm{E}-12$ & $2.66 \mathrm{E}-12$ & $2.66 \mathrm{E}-12$ & $2.66 \mathrm{E}-12$ & $2.66 \mathrm{E}-12$ & $2.66 \mathrm{E}-12$ & $2.66 \mathrm{E}-12$ & $2.66 \mathrm{E}-12$ & $2.66 \mathrm{E}-12$ \\
\hline SR 89 & $1.39 \mathrm{E}-12$ & $1.37 \mathrm{E}-12$ & $1.36 \mathrm{E}-12$ & $1.21 \mathrm{E}-12$ & $9.23 \mathrm{E}-13$ & $4.05 \mathrm{E}-13$ & $2.34 \mathrm{E}-13$ & $1.18 \mathrm{E}-13$ & $9.95 \mathrm{E}-15$ \\
\hline SR 90 & $8.45 \mathrm{E}-14$ & $8.45 \mathrm{E}-14$ & $8.45 \mathrm{E}-14$ & $8.45 \mathrm{E}-14$ & $8.44 \mathrm{E}-14$ & $8.40 \mathrm{E}-14$ & $8.38 \mathrm{E}-14$ & $8.35 \mathrm{E}-14$ & $8.26 \mathrm{E}-14$ \\
\hline SR 91 & $3.33 \mathrm{E}-16$ & $5.78 \mathrm{E}-17$ & $1.00 \mathrm{E}-17$ & $8.27 \mathrm{E}-24$ & $5.10 \mathrm{E}-39$ & $0.00 \mathrm{E}+00$ & $0.00 \mathrm{E}+00$ & $0.00 \mathrm{E}+00$ & $0.00 \mathrm{E}+00$ \\
\hline Y 89 & $5.89 \mathrm{E}-08$ & $5.92 \mathrm{E}-08$ & $5.95 \mathrm{E}-08$ & $6.03 \mathrm{E}-08$ & $6.05 \mathrm{E}-08$ & $6.05 \mathrm{E}-08$ & $6.05 \mathrm{E}-08$ & $6.05 \mathrm{E}-08$ & $6.05 \mathrm{E}-08$ \\
\hline Y 89M & $8.99 \mathrm{E}-14$ & $7.27 \mathrm{E}-14$ & $5.88 \mathrm{E}-14$ & $1.08 \mathrm{E}-14$ & $1.55 \mathrm{E}-16$ & 4.62E-22 & $9.57 \mathrm{E}-26$ & $2.38 \mathrm{E}-30$ & $0.00 \mathrm{E}+00$ \\
\hline Y 90 & $1.94 \mathrm{E}-10$ & $1.50 \mathrm{E}-10$ & $1.16 \mathrm{E}-10$ & $1.45 \mathrm{E}-11$ & $7.98 \mathrm{E}-14$ & $2.11 \mathrm{E}-17$ & $2.10 \mathrm{E}-17$ & $2.10 \mathrm{E}-17$ & $2.07 \mathrm{E}-17$ \\
\hline Y 90M & $8.95 \mathrm{E}-17$ & $4.18 \mathrm{E}-19$ & $1.95 \mathrm{E}-21$ & $4.42 \mathrm{E}-40$ & $0.00 \mathrm{E}+00$ & $0.00 \mathrm{E}+00$ & $0.00 \mathrm{E}+00$ & $0.00 \mathrm{E}+00$ & $0.00 \mathrm{E}+00$ \\
\hline Y 91 & $6.36 \mathrm{E}-13$ & $6.28 \mathrm{E}-13$ & $6.21 \mathrm{E}-13$ & $5.65 \mathrm{E}-13$ & $4.46 \mathrm{E}-13$ & $2.19 \mathrm{E}-13$ & $1.36 \mathrm{E}-13$ & $7.54 \mathrm{E}-14$ & $8.93 \mathrm{E}-15$ \\
\hline Y 92 & $1.61 \mathrm{E}-14$ & $1.47 \mathrm{E}-16$ & $1.33 \mathrm{E}-18$ & $6.20 \mathrm{E}-35$ & $0.00 \mathrm{E}+00$ & $0.00 \mathrm{E}+00$ & $0.00 \mathrm{E}+00$ & $0.00 \mathrm{E}+00$ & $0.00 \mathrm{E}+00$ \\
\hline ZR 89 & $1.58 \mathrm{E}-09$ & $1.28 \mathrm{E}-09$ & $1.04 \mathrm{E}-09$ & $1.90 \mathrm{E}-10$ & $2.73 \mathrm{E}-12$ & $8.14 \mathrm{E}-18$ & $1.68 \mathrm{E}-21$ & $4.18 \mathrm{E}-26$ & $1.12 \mathrm{E}-42$ \\
\hline ZR 90 & 8.89E-09 & 8.94E-09 & 8.97E-09 & 9.07E-09 & 9.09E-09 & $9.09 \mathrm{E}-09$ & $9.09 \mathrm{E}-09$ & $9.09 \mathrm{E}-09$ & $9.09 \mathrm{E}-09$ \\
\hline ZR 91 & $8.22 \mathrm{E}-07$ & $8.22 \mathrm{E}-07$ & $8.22 \mathrm{E}-07$ & $8.22 \mathrm{E}-07$ & $8.22 \mathrm{E}-07$ & $8.22 \mathrm{E}-07$ & $8.22 \mathrm{E}-07$ & $8.22 \mathrm{E}-07$ & $8.22 \mathrm{E}-07$ \\
\hline ZR 92 & $1.92 \mathrm{E}-05$ & $1.93 \mathrm{E}-05$ & $1.93 \mathrm{E}-05$ & $1.97 \mathrm{E}-05$ & $2.01 \mathrm{E}-05$ & $2.02 \mathrm{E}-05$ & $2.02 \mathrm{E}-05$ & $2.02 \mathrm{E}-05$ & $2.02 \mathrm{E}-05$ \\
\hline ZR 93 & 7.93E-07 & 7.93E-07 & $7.93 \mathrm{E}-07$ & $7.93 \mathrm{E}-07$ & 7.93E-07 & $7.93 \mathrm{E}-07$ & 7.93E-07 & 7.93E-07 & $7.93 \mathrm{E}-07$ \\
\hline ZR 94 & $1.74 \mathrm{E}-06$ & $1.74 \mathrm{E}-06$ & $1.74 \mathrm{E}-06$ & $1.74 \mathrm{E}-06$ & $1.74 \mathrm{E}-06$ & $1.74 \mathrm{E}-06$ & $1.74 \mathrm{E}-06$ & $1.74 \mathrm{E}-06$ & $1.74 \mathrm{E}-06$ \\
\hline ZR 95 & $7.65 \mathrm{E}-08$ & $7.56 \mathrm{E}-08$ & 7.48E-08 & $6.86 \mathrm{E}-08$ & $5.53 \mathrm{E}-08$ & $2.88 \mathrm{E}-08$ & $1.87 \mathrm{E}-08$ & $1.09 \mathrm{E}-08$ & $1.55 \mathrm{E}-09$ \\
\hline ZR 96 & $1.43 \mathrm{E}-10$ & $1.43 \mathrm{E}-10$ & $1.43 \mathrm{E}-10$ & $1.43 \mathrm{E}-10$ & $1.43 \mathrm{E}-10$ & $1.43 \mathrm{E}-10$ & $1.43 \mathrm{E}-10$ & $1.43 \mathrm{E}-10$ & $1.43 \mathrm{E}-10$ \\
\hline ZR 97 & $2.94 \mathrm{E}-11$ & $1.10 \mathrm{E}-11$ & $4.10 \mathrm{E}-12$ & $1.56 \mathrm{E}-15$ & $4.39 \mathrm{E}-24$ & $0.00 \mathrm{E}+00$ & $0.00 \mathrm{E}+00$ & $0.00 \mathrm{E}+00$ & $0.00 \mathrm{E}+00$ \\
\hline NB 92 & $9.98 \mathrm{E}-07$ & $9.32 \mathrm{E}-07$ & $8.71 \mathrm{E}-07$ & $5.05 \mathrm{E}-07$ & $1.29 \mathrm{E}-07$ & $2.15 \mathrm{E}-09$ & $1.40 \mathrm{E}-10$ & $4.63 \mathrm{E}-12$ & $2.15 \mathrm{E}-17$ \\
\hline NB 93 & $3.45 \mathrm{E}-02$ & $3.45 \mathrm{E}-02$ & $3.45 \mathrm{E}-02$ & $3.45 \mathrm{E}-02$ & $3.45 \mathrm{E}-02$ & $3.45 \mathrm{E}-02$ & $3.45 \mathrm{E}-02$ & $3.45 \mathrm{E}-02$ & $3.45 \mathrm{E}-02$ \\
\hline NB 93M & $1.58 \mathrm{E}-13$ & $1.59 \mathrm{E}-13$ & $1.59 \mathrm{E}-13$ & $1.67 \mathrm{E}-13$ & $1.85 \mathrm{E}-13$ & $2.39 \mathrm{E}-13$ & $2.75 \mathrm{E}-13$ & $3.20 \mathrm{E}-13$ & $4.78 \mathrm{E}-13$ \\
\hline NB 94 & $2.03 \mathrm{E}-04$ & $2.03 \mathrm{E}-04$ & $2.03 \mathrm{E}-04$ & $2.03 \mathrm{E}-04$ & $2.03 \mathrm{E}-04$ & $2.03 \mathrm{E}-04$ & $2.03 \mathrm{E}-04$ & $2.03 \mathrm{E}-04$ & $2.03 \mathrm{E}-04$ \\
\hline NB 95 & $2.48 \mathrm{E}-06$ & $2.43 \mathrm{E}-06$ & $2.38 \mathrm{E}-06$ & $2.04 \mathrm{E}-06$ & $1.39 \mathrm{E}-06$ & $4.39 \mathrm{E}-07$ & $2.06 \mathrm{E}-07$ & $8.18 \mathrm{E}-08$ & $3.86 \mathrm{E}-09$ \\
\hline NB 95M & $3.07 \mathrm{E}-11$ & $3.06 \mathrm{E}-11$ & $3.04 \mathrm{E}-11$ & $2.85 \mathrm{E}-11$ & $2.31 \mathrm{E}-11$ & $1.21 \mathrm{E}-11$ & $7.83 \mathrm{E}-12$ & $4.55 \mathrm{E}-12$ & $6.48 \mathrm{E}-13$ \\
\hline NB 96 & $3.91 \mathrm{E}-09$ & $1.92 \mathrm{E}-09$ & $9.39 \mathrm{E}-10$ & $3.14 \mathrm{E}-12$ & $2.04 \mathrm{E}-18$ & $5.56 \mathrm{E}-37$ & $0.00 \mathrm{E}+00$ & $0.00 \mathrm{E}+00$ & $0.00 \mathrm{E}+00$ \\
\hline
\end{tabular}


BSU-8242 3 DPA As-Run Physics Analysis

\begin{tabular}{|c|c|c|c|c|c|c|c|c|c|}
\hline \multicolumn{10}{|c|}{ ACTIVATION PRODUCTS (grams) } \\
\hline ISOTOPE & EOC & 1 Day & 2 Days & 10 Days & 30 Days & 90 Days & 130 Days & 180 Days & 360 Days \\
\hline NB 97 & $7.43 \mathrm{E}-11$ & $7.84 \mathrm{E}-13$ & $2.93 \mathrm{E}-13$ & $1.20 \mathrm{E}-16$ & $3.37 \mathrm{E}-25$ & $0.00 \mathrm{E}+00$ & $0.00 \mathrm{E}+00$ & $0.00 \mathrm{E}+00$ & $0.00 \mathrm{E}+00$ \\
\hline NB 97M & $2.74 \mathrm{E}-14$ & $1.03 \mathrm{E}-14$ & $3.83 \mathrm{E}-15$ & $1.46 \mathrm{E}-18$ & $4.11 \mathrm{E}-27$ & $0.00 \mathrm{E}+00$ & $0.00 \mathrm{E}+00$ & $0.00 \mathrm{E}+00$ & $0.00 \mathrm{E}+00$ \\
\hline NB 98 & 7.23E-15 & $0.00 \mathrm{E}+00$ & $0.00 \mathrm{E}+00$ & $0.00 \mathrm{E}+00$ & $0.00 \mathrm{E}+00$ & $0.00 \mathrm{E}+00$ & $0.00 \mathrm{E}+00$ & $0.00 \mathrm{E}+00$ & $0.00 \mathrm{E}+00$ \\
\hline NB100 & $3.14 \mathrm{E}-16$ & $0.00 \mathrm{E}+00$ & $0.00 \mathrm{E}+00$ & $0.00 \mathrm{E}+00$ & $0.00 \mathrm{E}+00$ & $0.00 \mathrm{E}+00$ & $0.00 \mathrm{E}+00$ & $0.00 \mathrm{E}+00$ & $0.00 \mathrm{E}+00$ \\
\hline MO 92 & $4.51 \mathrm{E}-01$ & $4.51 \mathrm{E}-01$ & $4.51 \mathrm{E}-01$ & $4.51 \mathrm{E}-01$ & $4.51 \mathrm{E}-01$ & $4.51 \mathrm{E}-01$ & $4.51 \mathrm{E}-01$ & $4.51 \mathrm{E}-01$ & $4.51 \mathrm{E}-01$ \\
\hline MO 93M & $8.35 \mathrm{E}-09$ & $7.36 \mathrm{E}-10$ & $6.49 \mathrm{E}-11$ & $2.37 \mathrm{E}-19$ & $1.91 \mathrm{E}-40$ & $0.00 \mathrm{E}+00$ & $0.00 \mathrm{E}+00$ & $0.00 \mathrm{E}+00$ & $0.00 \mathrm{E}+00$ \\
\hline MO 93 & $1.13 \mathrm{E}-04$ & $1.13 \mathrm{E}-04$ & $1.13 \mathrm{E}-04$ & $1.13 \mathrm{E}-04$ & $1.13 \mathrm{E}-04$ & $1.13 \mathrm{E}-04$ & $1.13 \mathrm{E}-04$ & $1.13 \mathrm{E}-04$ & $1.13 \mathrm{E}-04$ \\
\hline MO 94 & $2.90 \mathrm{E}-01$ & $2.90 \mathrm{E}-01$ & $2.90 \mathrm{E}-01$ & $2.90 \mathrm{E}-01$ & $2.90 \mathrm{E}-01$ & $2.90 \mathrm{E}-01$ & $2.90 \mathrm{E}-01$ & $2.90 \mathrm{E}-01$ & $2.90 \mathrm{E}-01$ \\
\hline MO 95 & 4.71E-01 & 4.71E-01 & 4.71E-01 & 4.71E-01 & 4.71E-01 & 4.71E-01 & 4.71E-01 & $4.71 \mathrm{E}-01$ & $4.71 \mathrm{E}-01$ \\
\hline MO 96 & $5.56 \mathrm{E}-01$ & $5.56 \mathrm{E}-01$ & $5.56 \mathrm{E}-01$ & $5.56 \mathrm{E}-01$ & $5.56 \mathrm{E}-01$ & $5.56 \mathrm{E}-01$ & $5.56 \mathrm{E}-01$ & $5.56 \mathrm{E}-01$ & $5.56 \mathrm{E}-01$ \\
\hline MO 97 & $3.11 \mathrm{E}-01$ & $3.11 \mathrm{E}-01$ & $3.11 \mathrm{E}-01$ & $3.11 \mathrm{E}-01$ & $3.11 \mathrm{E}-01$ & $3.11 \mathrm{E}-01$ & $3.11 \mathrm{E}-01$ & $3.11 \mathrm{E}-01$ & $3.11 \mathrm{E}-01$ \\
\hline MO 98 & 7.84E-01 & $7.84 \mathrm{E}-01$ & $7.84 \mathrm{E}-01$ & $7.84 \mathrm{E}-01$ & $7.84 \mathrm{E}-01$ & $7.84 \mathrm{E}-01$ & $7.84 \mathrm{E}-01$ & $7.84 \mathrm{E}-01$ & $7.84 \mathrm{E}-01$ \\
\hline MO 99 & $5.99 \mathrm{E}-05$ & $4.66 \mathrm{E}-05$ & $3.62 \mathrm{E}-05$ & $4.82 \mathrm{E}-06$ & $3.12 \mathrm{E}-08$ & $8.43 \mathrm{E}-15$ & $3.53 \mathrm{E}-19$ & $1.19 \mathrm{E}-24$ & $0.00 \mathrm{E}+00$ \\
\hline MO100 & $3.18 \mathrm{E}-01$ & $3.18 \mathrm{E}-01$ & $3.18 \mathrm{E}-01$ & $3.18 \mathrm{E}-01$ & $3.18 \mathrm{E}-01$ & $3.18 \mathrm{E}-01$ & $3.18 \mathrm{E}-01$ & $3.18 \mathrm{E}-01$ & $3.18 \mathrm{E}-01$ \\
\hline MO101 & $5.61 \mathrm{E}-08$ & $0.00 \mathrm{E}+00$ & $0.00 \mathrm{E}+00$ & $0.00 \mathrm{E}+00$ & $0.00 \mathrm{E}+00$ & $0.00 \mathrm{E}+00$ & $0.00 \mathrm{E}+00$ & $0.00 \mathrm{E}+00$ & $0.00 \mathrm{E}+00$ \\
\hline TC 99 & $3.11 \mathrm{E}-04$ & $3.12 \mathrm{E}-04$ & $3.14 \mathrm{E}-04$ & $3.17 \mathrm{E}-04$ & $3.18 \mathrm{E}-04$ & $3.18 \mathrm{E}-04$ & $3.18 \mathrm{E}-04$ & $3.18 \mathrm{E}-04$ & $3.18 \mathrm{E}-04$ \\
\hline TC100 & $6.09 \mathrm{E}-11$ & $0.00 \mathrm{E}+00$ & $0.00 \mathrm{E}+00$ & $0.00 \mathrm{E}+00$ & $0.00 \mathrm{E}+00$ & $0.00 \mathrm{E}+00$ & $0.00 \mathrm{E}+00$ & $0.00 \mathrm{E}+00$ & $0.00 \mathrm{E}+00$ \\
\hline TC101 & $5.45 \mathrm{E}-08$ & $0.00 \mathrm{E}+00$ & $0.00 \mathrm{E}+00$ & $0.00 \mathrm{E}+00$ & $0.00 \mathrm{E}+00$ & $0.00 \mathrm{E}+00$ & $0.00 \mathrm{E}+00$ & $0.00 \mathrm{E}+00$ & $0.00 \mathrm{E}+00$ \\
\hline RU 99 & $4.87 \mathrm{E}-10$ & $4.90 \mathrm{E}-10$ & $4.93 \mathrm{E}-10$ & $5.15 \mathrm{E}-10$ & $5.72 \mathrm{E}-10$ & $7.42 \mathrm{E}-10$ & $8.55 \mathrm{E}-10$ & $9.97 \mathrm{E}-10$ & $1.51 \mathrm{E}-09$ \\
\hline RU100 & $2.12 \mathrm{E}-05$ & $2.12 \mathrm{E}-05$ & $2.12 \mathrm{E}-05$ & $2.12 \mathrm{E}-05$ & $2.12 \mathrm{E}-05$ & $2.12 \mathrm{E}-05$ & $2.12 \mathrm{E}-05$ & $2.12 \mathrm{E}-05$ & $2.12 \mathrm{E}-05$ \\
\hline RU101 & $6.77 \mathrm{E}-04$ & $6.77 \mathrm{E}-04$ & $6.77 \mathrm{E}-04$ & $6.77 \mathrm{E}-04$ & $6.77 \mathrm{E}-04$ & $6.77 \mathrm{E}-04$ & $6.77 \mathrm{E}-04$ & $6.77 \mathrm{E}-04$ & $6.77 \mathrm{E}-04$ \\
\hline RU102 & $1.47 \mathrm{E}-05$ & $1.47 \mathrm{E}-05$ & $1.47 \mathrm{E}-05$ & $1.47 \mathrm{E}-05$ & $1.47 \mathrm{E}-05$ & $1.47 \mathrm{E}-05$ & $1.47 \mathrm{E}-05$ & $1.47 \mathrm{E}-05$ & $1.47 \mathrm{E}-05$ \\
\hline RU103 & $9.39 \mathrm{E}-09$ & $9.22 \mathrm{E}-09$ & $9.06 \mathrm{E}-09$ & 7.87E-09 & $5.53 \mathrm{E}-09$ & $1.92 \mathrm{E}-09$ & $9.47 \mathrm{E}-10$ & $3.92 \mathrm{E}-10$ & $1.64 \mathrm{E}-11$ \\
\hline RU104 & $9.26 \mathrm{E}-11$ & $9.26 \mathrm{E}-11$ & $9.26 \mathrm{E}-11$ & $9.26 \mathrm{E}-11$ & $9.26 \mathrm{E}-11$ & $9.26 \mathrm{E}-11$ & $9.26 \mathrm{E}-11$ & $9.26 \mathrm{E}-11$ & $9.26 \mathrm{E}-11$ \\
\hline RU105 & $5.26 \mathrm{E}-16$ & $1.24 \mathrm{E}-17$ & $2.92 \mathrm{E}-19$ & $2.79 \mathrm{E}-32$ & $0.00 \mathrm{E}+00$ & $0.00 \mathrm{E}+00$ & $0.00 \mathrm{E}+00$ & $0.00 \mathrm{E}+00$ & $0.00 \mathrm{E}+00$ \\
\hline RH103 & $8.19 \mathrm{E}-10$ & $8.36 \mathrm{E}-10$ & $8.52 \mathrm{E}-10$ & $9.70 \mathrm{E}-10$ & $1.20 \mathrm{E}-09$ & $1.56 \mathrm{E}-09$ & $1.66 \mathrm{E}-09$ & $1.71 \mathrm{E}-09$ & $1.75 \mathrm{E}-09$ \\
\hline
\end{tabular}


BSU-8242 3 DPA As-Run Physics Analysis

\begin{tabular}{|c|c|c|c|c|c|c|c|c|c|}
\hline \multicolumn{10}{|c|}{ ACTIVATION PRODUCTS (grams) } \\
\hline ISOTOPE & EOC & 1 Day & 2 Days & 10 Days & 30 Days & 90 Days & 130 Days & 180 Days & 360 Days \\
\hline RH104 & $1.87 \mathrm{E}-15$ & $0.00 \mathrm{E}+00$ & $0.00 \mathrm{E}+00$ & $0.00 \mathrm{E}+00$ & $0.00 \mathrm{E}+00$ & $0.00 \mathrm{E}+00$ & $0.00 \mathrm{E}+00$ & $0.00 \mathrm{E}+00$ & $0.00 \mathrm{E}+00$ \\
\hline RH104M & $32 \mathrm{E}-16$ & $0.00 \mathrm{E}+00$ & $0.00 \mathrm{E}+00$ & $0.00 \mathrm{E}+00$ & $00 \mathrm{E}+00$ & $0.00 \mathrm{E}+00$ & $0.00 \mathrm{E}+00$ & $0.00 \mathrm{E}+00$ & $0.00 \mathrm{E}+00$ \\
\hline RH105 & $3.34 \mathrm{E}-15$ & $2.45 \mathrm{E}-15$ & $1.54 \mathrm{E}-15$ & $3.57 \mathrm{E}-17$ & $2.93 \mathrm{E}-21$ & $1.61 \mathrm{E}-33$ & $1.09 \mathrm{E}-41$ & $0.00 \mathrm{E}+00$ & $0.00 \mathrm{E}+00$ \\
\hline RH106N & $1.78 \mathrm{E}-17$ & $9.23 \mathrm{E}-21$ & $4.80 \mathrm{E}-24$ & $0.00 \mathrm{E}+00$ & $0.00 \mathrm{E}+00$ & $0.00 \mathrm{E}+00$ & $0.00 \mathrm{E}+00$ & $0.00 \mathrm{E}+00$ & $0.00 \mathrm{E}+00$ \\
\hline PD104 & $1.22 \mathrm{E}-10$ & $1.22 \mathrm{E}-10$ & $1.22 \mathrm{E}-10$ & $1.22 \mathrm{E}-10$ & & & & 1.22 & \\
\hline & & & & & & & & & \\
\hline & $1.74 \mathrm{E}-14$ & 174514 & $1.74 \mathrm{E}-14$ & $1.74 \mathrm{E}-14$ & $74 \mathrm{E}-14$ & $1.74 \mathrm{E}-14$ & $74 \mathrm{E}-14$ & $1.74 \mathrm{E}-14$ & e-14 \\
\hline PD107 & E-17 & $1.20 \mathrm{E}-17$ & $1.20 \mathrm{E}-17$ & $1.20 \mathrm{E}-17$ & $20 \mathrm{E}-17$ & $1.20 \mathrm{E}-17$ & $1.20 \mathrm{E}-17$ & $1.20 \mathrm{E}-17$ & $1.20 \mathrm{E}-17$ \\
\hline TM172 & $5.79 \mathrm{E}-15$ & $4.46 \mathrm{E}-15$ & $3.43 \mathrm{E}-15$ & $4.24 \mathrm{E}-16$ & $2.26 \mathrm{E}-18$ & $3.46 \mathrm{E}-25$ & $9.89 \mathrm{E}-30$ & $2.07 \mathrm{E}-35$ & $0.00 \mathrm{E}+00$ \\
\hline TM173 & $6.46 \mathrm{E}-16$ & $8.58 \mathrm{E}-17$ & $1.14 \mathrm{E}-17$ & $1.10 \mathrm{E}-24$ & 3.15 & $0.00 \mathrm{E}+00$ & $0.00 \mathrm{E}+00$ & $0.00 \mathrm{E}+00$ & $0.00 \mathrm{E}+00$ \\
\hline YB172 & & & & & & & & & \\
\hline & & & & & & & & & \\
\hline & & & & & & & 15 & & -15 \\
\hline YB175 & 1.10 & 9.3 & $7.89 \mathrm{E}-18$ & 2.10 & 7.68 & $3.75 \mathrm{E}-24$ & $0.00 \mathrm{E}+00$ & $0.00 \mathrm{E}+00$ & $0.00 \mathrm{E}+00$ \\
\hline LU175 & $3.40 \mathrm{E}-06$ & $3.42 \mathrm{E}-06$ & 3.44E-06 & 3.57E-06 & 3.87E-06 & 4.47E-06 & 4.71E-06 & 4.91E-06 & $5.16 \mathrm{E}-06$ \\
\hline LU176 & & & $8.97 \mathrm{E}-08$ & & $8.97 \mathrm{E}-08$ & $8.97 \mathrm{E}-08$ & $8.97 \mathrm{E}-08$ & $8.97 \mathrm{E}-08$ & $8.97 \mathrm{E}-08$ \\
\hline & & & & & & & & & \\
\hline LU177 & & & & 09 & & -12 & -12 & -12 & $5.29 \mathrm{E}-13$ \\
\hline LU177M & $2.66 \mathrm{E}-10$ & $2.65 \mathrm{E}-10$ & $2.64 \mathrm{E}-10$ & $2.54 \mathrm{E}-10$ & $2.33 \mathrm{E}-10$ & $1.78 \mathrm{E}-10$ & 1.49E-10 & 1.19E-10 & $5.32 \mathrm{E}-11$ \\
\hline HF174 & $6.71 \mathrm{E}-06$ & $6.71 \mathrm{E}-06$ & $6.71 \mathrm{E}-06$ & $6.71 \mathrm{E}-06$ & $6.71 \mathrm{E}-06$ & $6.71 \mathrm{E}-06$ & $6.71 \mathrm{E}-06$ & $6.71 \mathrm{E}-06$ & $6.71 \mathrm{E}-06$ \\
\hline HF175 & $1.81 \mathrm{E}-06$ & 1.79E-06 & $1.77 \mathrm{E}-06$ & $1.64 \mathrm{E}-06$ & $1.34 \mathrm{E}-06$ & 7.42E-07 & 4.99E-07 & 3.04E-07 & $5.12 \mathrm{E}-08$ \\
\hline HF176 & & & & & & & & & 3.07E-04 \\
\hline HF177 & & & & & & & & 1.03E-04 & $1.03 \mathrm{E}-04$ \\
\hline HF178 & $1.85 \mathrm{E}-03$ & $1.85 \mathrm{E}-03$ & $1.85 \mathrm{E}-03$ & $1.85 \mathrm{E}-03$ & $1.85 \mathrm{E}-03$ & $1.85 \mathrm{E}-03$ & $1.85 \mathrm{E}-03$ & $1.85 \mathrm{E}-03$ & $1.85 \mathrm{E}-03$ \\
\hline HF178M & $3.49 \mathrm{E}-13$ & $0.00 \mathrm{E}+00$ & $0.00 \mathrm{E}+00$ & $0.00 \mathrm{E}+00$ & $0.00 \mathrm{E}+00$ & $0.00 \mathrm{E}+00$ & $0.00 \mathrm{E}+00$ & $0.00 \mathrm{E}+00$ & $0.00 \mathrm{E}+00$ \\
\hline HF179 & $2.44 \mathrm{E}-03$ & $2.44 \mathrm{E}-03$ & $2.44 \mathrm{E}-03$ & $2.44 \mathrm{E}-03$ & $2.44 \mathrm{E}-03$ & $2.44 \mathrm{E}-03$ & $2.44 \mathrm{E}-03$ & $2.44 \mathrm{E}-03$ & $2.44 \mathrm{E}-03$ \\
\hline
\end{tabular}


BSU-8242 3 DPA As-Run Physics Analysis

\begin{tabular}{|c|c|c|c|c|c|c|c|c|c|}
\hline \multicolumn{10}{|c|}{ ACTIVATION PRODUCTS (grams) } \\
\hline ISOTOPE & EOC & 1 Day & 2 Days & 10 Days & 30 Days & 90 Days & 130 Days & 180 Days & 360 Days \\
\hline HF179M & 1.57E-09 & $0.00 \mathrm{E}+00$ & $0.00 \mathrm{E}+00$ & $0.00 \mathrm{E}+00$ & $0.00 \mathrm{E}+00$ & $0.00 \mathrm{E}+00$ & $0.00 \mathrm{E}+00$ & $0.00 \mathrm{E}+00$ & $0.00 \mathrm{E}+00$ \\
\hline HF180 & $3.25 \mathrm{E}-03$ & $3.25 \mathrm{E}-03$ & $3.25 \mathrm{E}-03$ & $3.25 \mathrm{E}-03$ & $3.25 \mathrm{E}-03$ & $3.25 \mathrm{E}-03$ & $3.25 \mathrm{E}-03$ & $3.25 \mathrm{E}-03$ & $3.25 \mathrm{E}-03$ \\
\hline HF180M & $9.14 \mathrm{E}-09$ & $4.44 \mathrm{E}-10$ & $2.16 \mathrm{E}-11$ & $6.69 \mathrm{E}-22$ & $0.00 \mathrm{E}+00$ & $0.00 \mathrm{E}+00$ & $0.00 \mathrm{E}+00$ & $0.00 \mathrm{E}+00$ & $0.00 \mathrm{E}+00$ \\
\hline HF181 & $2.46 \mathrm{E}-05$ & $2.42 \mathrm{E}-05$ & $2.38 \mathrm{E}-05$ & 2.09E-05 & $1.51 \mathrm{E}-05$ & $5.65 \mathrm{E}-06$ & $2.94 \mathrm{E}-06$ & $1.30 \mathrm{E}-06$ & $6.84 \mathrm{E}-08$ \\
\hline HF182 & $7.33 \mathrm{E}-07$ & $7.33 \mathrm{E}-07$ & $7.33 \mathrm{E}-07$ & 7.33E-07 & 7.33E-07 & 7.33E-07 & 7.33E-07 & 7.33E-07 & 7.33E-07 \\
\hline TA180 & $1.90 \mathrm{E}-04$ & $1.90 \mathrm{E}-04$ & $1.90 \mathrm{E}-04$ & $1.90 \mathrm{E}-04$ & $1.90 \mathrm{E}-04$ & $1.90 \mathrm{E}-04$ & $1.90 \mathrm{E}-04$ & $1.90 \mathrm{E}-04$ & $1.90 \mathrm{E}-04$ \\
\hline TA181 & $3.39 \mathrm{E}+00$ & $3.39 \mathrm{E}+00$ & $3.39 \mathrm{E}+00$ & $3.39 \mathrm{E}+00$ & $3.39 \mathrm{E}+00$ & $3.39 \mathrm{E}+00$ & $3.39 \mathrm{E}+00$ & $3.39 \mathrm{E}+00$ & $3.39 \mathrm{E}+00$ \\
\hline TA182 & $8.74 \mathrm{E}-02$ & $8.69 \mathrm{E}-02$ & $8.64 \mathrm{E}-02$ & $8.23 \mathrm{E}-02$ & $7.30 \mathrm{E}-02$ & $5.08 \mathrm{E}-02$ & $3.99 \mathrm{E}-02$ & $2.96 \mathrm{E}-02$ & $9.99 \mathrm{E}-03$ \\
\hline TA182M & $4.26 \mathrm{E}-08$ & $0.00 \mathrm{E}+00$ & $0.00 \mathrm{E}+00$ & $0.00 \mathrm{E}+00$ & $0.00 \mathrm{E}+00$ & $0.00 \mathrm{E}+00$ & $0.00 \mathrm{E}+00$ & $0.00 \mathrm{E}+00$ & $0.00 \mathrm{E}+00$ \\
\hline TA183 & $3.65 \mathrm{E}-02$ & $3.18 \mathrm{E}-02$ & $2.78 \mathrm{E}-02$ & $9.36 \mathrm{E}-03$ & $6.18 \mathrm{E}-04$ & $1.78 \mathrm{E}-07$ & $7.73 \mathrm{E}-10$ & $8.59 \mathrm{E}-13$ & $2.04 \mathrm{E}-23$ \\
\hline W180 & $1.64 \mathrm{E}-17$ & $1.65 \mathrm{E}-17$ & $1.65 \mathrm{E}-17$ & $1.67 \mathrm{E}-17$ & $1.71 \mathrm{E}-17$ & $1.85 \mathrm{E}-17$ & $1.94 \mathrm{E}-17$ & $2.05 \mathrm{E}-17$ & $2.45 \mathrm{E}-17$ \\
\hline W182 & $1.59 \mathrm{E}-01$ & $1.59 \mathrm{E}-01$ & $1.60 \mathrm{E}-01$ & $1.64 \mathrm{E}-01$ & $1.73 \mathrm{E}-01$ & $1.95 \mathrm{E}-01$ & $2.06 \mathrm{E}-01$ & $2.17 \mathrm{E}-01$ & $2.36 \mathrm{E}-01$ \\
\hline W183M & $5.10 \mathrm{E}-11$ & $0.00 \mathrm{E}+00$ & $0.00 \mathrm{E}+00$ & $0.00 \mathrm{E}+00$ & $0.00 \mathrm{E}+00$ & $0.00 \mathrm{E}+00$ & $0.00 \mathrm{E}+00$ & $0.00 \mathrm{E}+00$ & $0.00 \mathrm{E}+00$ \\
\hline W183 & $8.57 \mathrm{E}-01$ & $8.62 \mathrm{E}-01$ & $8.66 \mathrm{E}-01$ & $8.84 \mathrm{E}-01$ & $8.93 \mathrm{E}-01$ & $8.93 \mathrm{E}-01$ & $8.93 \mathrm{E}-01$ & $8.93 \mathrm{E}-01$ & $8.93 \mathrm{E}-01$ \\
\hline W184 & $5.61 \mathrm{E}-02$ & $5.61 \mathrm{E}-02$ & $5.61 \mathrm{E}-02$ & $5.61 \mathrm{E}-02$ & $5.61 \mathrm{E}-02$ & $5.61 \mathrm{E}-02$ & $5.61 \mathrm{E}-02$ & $5.61 \mathrm{E}-02$ & $5.61 \mathrm{E}-02$ \\
\hline W185 & $8.48 \mathrm{E}-05$ & $8.40 \mathrm{E}-05$ & 8.33E-05 & 7.73E-05 & $6.43 \mathrm{E}-05$ & $3.70 \mathrm{E}-05$ & $2.56 \mathrm{E}-05$ & $1.61 \mathrm{E}-05$ & $3.06 \mathrm{E}-06$ \\
\hline $\mathrm{W} 185 \mathrm{M}$ & $4.10 \mathrm{E}-12$ & $0.00 \mathrm{E}+00$ & $0.00 \mathrm{E}+00$ & $0.00 \mathrm{E}+00$ & $0.00 \mathrm{E}+00$ & $0.00 \mathrm{E}+00$ & $0.00 \mathrm{E}+00$ & $0.00 \mathrm{E}+00$ & $0.00 \mathrm{E}+00$ \\
\hline W186 & $3.92 \mathrm{E}-07$ & $4.01 \mathrm{E}-07$ & $4.08 \mathrm{E}-07$ & $4.36 \mathrm{E}-07$ & $4.45 \mathrm{E}-07$ & $4.45 \mathrm{E}-07$ & $4.45 \mathrm{E}-07$ & $4.45 \mathrm{E}-07$ & $4.45 \mathrm{E}-07$ \\
\hline W187 & $6.94 \mathrm{E}-10$ & $3.46 \mathrm{E}-10$ & $1.73 \mathrm{E}-10$ & $6.59 \mathrm{E}-13$ & $5.93 \mathrm{E}-19$ & $4.32 \mathrm{E}-37$ & $0.00 \mathrm{E}+00$ & $0.00 \mathrm{E}+00$ & $0.00 \mathrm{E}+00$ \\
\hline W188 & $1.08 \mathrm{E}-10$ & $1.07 \mathrm{E}-10$ & $1.06 \mathrm{E}-10$ & $9.81 \mathrm{E}-11$ & $8.03 \mathrm{E}-11$ & $4.41 \mathrm{E}-11$ & $2.96 \mathrm{E}-11$ & $1.80 \mathrm{E}-11$ & $2.98 \mathrm{E}-12$ \\
\hline RE185 & $3.56 \mathrm{E}-05$ & $3.64 \mathrm{E}-05$ & $3.72 \mathrm{E}-05$ & $4.31 \mathrm{E}-05$ & $5.61 \mathrm{E}-05$ & $8.35 \mathrm{E}-05$ & 9.49E-05 & $1.04 \mathrm{E}-04$ & $1.17 \mathrm{E}-04$ \\
\hline RE186 & $8.17 \mathrm{E}-07$ & $6.80 \mathrm{E}-07$ & $5.66 \mathrm{E}-07$ & $1.30 \mathrm{E}-07$ & $3.32 \mathrm{E}-09$ & $5.47 \mathrm{E}-14$ & $3.55 \mathrm{E}-17$ & $3.67 \mathrm{E}-21$ & $1.65 \mathrm{E}-35$ \\
\hline RE187 & $1.65 \mathrm{E}-08$ & $1.68 \mathrm{E}-08$ & $1.70 \mathrm{E}-08$ & $1.72 \mathrm{E}-08$ & $1.72 \mathrm{E}-08$ & $1.72 \mathrm{E}-08$ & $1.72 \mathrm{E}-08$ & $1.72 \mathrm{E}-08$ & $1.72 \mathrm{E}-08$ \\
\hline RE188 & $1.93 \mathrm{E}-11$ & $8.05 \mathrm{E}-12$ & $3.70 \mathrm{E}-12$ & $1.01 \mathrm{E}-12$ & $8.28 \mathrm{E}-13$ & $4.54 \mathrm{E}-13$ & $3.05 \mathrm{E}-13$ & $1.85 \mathrm{E}-13$ & $3.06 \mathrm{E}-14$ \\
\hline RE188M & $3.27 \mathrm{E}-13$ & $0.00 \mathrm{E}+00$ & $0.00 \mathrm{E}+00$ & $0.00 \mathrm{E}+00$ & $0.00 \mathrm{E}+00$ & $0.00 \mathrm{E}+00$ & $0.00 \mathrm{E}+00$ & $0.00 \mathrm{E}+00$ & $0.00 \mathrm{E}+00$ \\
\hline RE189 & $4.19 \mathrm{E}-16$ & $2.12 \mathrm{E}-16$ & $1.07 \mathrm{E}-16$ & $4.46 \mathrm{E}-19$ & $5.05 \mathrm{E}-25$ & $7.95 \mathrm{E}-43$ & $0.00 \mathrm{E}+00$ & $0.00 \mathrm{E}+00$ & $0.00 \mathrm{E}+00$ \\
\hline
\end{tabular}


BSU-8242 3 DPA As-Run Physics Analysis

\begin{tabular}{|c|c|c|c|c|c|c|c|c|c|}
\hline \multicolumn{10}{|c|}{ ACTIVATION PRODUCTS (grams) } \\
\hline ISOTOPE & EOC & 1 Day & 2 Days & 10 Days & 30 Days & 90 Days & 130 Days & 180 Days & 360 Days \\
\hline OS186 & $5.89 \mathrm{E}-06$ & $6.01 \mathrm{E}-06$ & $6.12 \mathrm{E}-06$ & $6.53 \mathrm{E}-06$ & $6.65 \mathrm{E}-06$ & $6.65 \mathrm{E}-06$ & $6.65 \mathrm{E}-06$ & $6.65 \mathrm{E}-06$ & $6.65 \mathrm{E}-06$ \\
\hline OS188 & $5.43 \mathrm{E}-10$ & $5.55 \mathrm{E}-10$ & $5.61 \mathrm{E}-10$ & $5.72 \mathrm{E}-10$ & $5.90 \mathrm{E}-10$ & $6.26 \mathrm{E}-10$ & $6.41 \mathrm{E}-10$ & $6.53 \mathrm{E}-10$ & $6.68 \mathrm{E}-10$ \\
\hline OS189 & $3.90 \mathrm{E}-12$ & $3.90 \mathrm{E}-12$ & $3.90 \mathrm{E}-12$ & $3.90 \mathrm{E}-12$ & $3.90 \mathrm{E}-12$ & $3.90 \mathrm{E}-12$ & $3.90 \mathrm{E}-12$ & $3.90 \mathrm{E}-12$ & $3.90 \mathrm{E}-12$ \\
\hline OS190 & $1.39 \mathrm{E}-13$ & $1.39 \mathrm{E}-13$ & $1.39 \mathrm{E}-13$ & $1.39 \mathrm{E}-13$ & $1.39 \mathrm{E}-13$ & $1.39 \mathrm{E}-13$ & $1.39 \mathrm{E}-13$ & $1.39 \mathrm{E}-13$ & $1.39 \mathrm{E}-13$ \\
\hline OS191 & $2.08 \mathrm{E}-16$ & $2.07 \mathrm{E}-16$ & $2.00 \mathrm{E}-16$ & $1.40 \mathrm{E}-16$ & $5.70 \mathrm{E}-17$ & $3.83 \mathrm{E}-18$ & $6.33 \mathrm{E}-19$ & $6.68 \mathrm{E}-20$ & $2.03 \mathrm{E}-23$ \\
\hline OS191M & $1.09 \mathrm{E}-17$ & $3.02 \mathrm{E}-18$ & $8.40 \mathrm{E}-19$ & $3.01 \mathrm{E}-23$ & $2.31 \mathrm{E}-34$ & $0.00 \mathrm{E}+00$ & $0.00 \mathrm{E}+00$ & $0.00 \mathrm{E}+00$ & $0.00 \mathrm{E}+00$ \\
\hline IR191 & $1.37 \mathrm{E}-16$ & $1.47 \mathrm{E}-16$ & $1.56 \mathrm{E}-16$ & $2.17 \mathrm{E}-16$ & $3.00 \mathrm{E}-16$ & $3.53 \mathrm{E}-16$ & $3.56 \mathrm{E}-16$ & $3.57 \mathrm{E}-16$ & $3.57 \mathrm{E}-16$ \\
\hline IR192 & $2.41 \mathrm{E}-17$ & 2.39E-17 & $2.36 \mathrm{E}-17$ & $2.19 \mathrm{E}-17$ & $1.82 \mathrm{E}-17$ & $1.04 \mathrm{E}-17$ & $7.13 \mathrm{E}-18$ & $4.46 \mathrm{E}-18$ & 8.27E-19 \\
\hline PT192 & $3.37 \mathrm{E}-18$ & $3.58 \mathrm{E}-18$ & $3.79 \mathrm{E}-18$ & $5.42 \mathrm{E}-18$ & 8.98E-18 & $1.64 \mathrm{E}-17$ & $1.95 \mathrm{E}-17$ & $2.21 \mathrm{E}-17$ & $2.55 \mathrm{E}-17$ \\
\hline SUMTOT & $8.53 \mathrm{E}+02$ & $8.53 \mathrm{E}+02$ & $8.53 \mathrm{E}+02$ & $8.53 \mathrm{E}+02$ & $8.53 \mathrm{E}+02$ & $8.53 \mathrm{E}+02$ & $8.53 \mathrm{E}+02$ & $8.53 \mathrm{E}+02$ & $8.53 \mathrm{E}+02$ \\
\hline 0TOTAL & $8.53 \mathrm{E}+02$ & $8.53 \mathrm{E}+02$ & $8.53 \mathrm{E}+02$ & $8.53 \mathrm{E}+02$ & $8.53 \mathrm{E}+02$ & $8.53 \mathrm{E}+02$ & $8.53 \mathrm{E}+02$ & $8.53 \mathrm{E}+02$ & $8.53 \mathrm{E}+02$ \\
\hline
\end{tabular}

Table 24. Source Terms for 'A8 400C 3 DPA X' Capsule.

\begin{tabular}{|c|c|c|c|c|c|c|c|c|c|}
\hline \multicolumn{10}{|c|}{ ACTIVATION PRODUCTS (grams) } \\
\hline ISOTOPE & EOC & 1 Day & 2 Days & 10 Days & 30 Days & 90 Days & 130 Days & 180 Days & 360 Days \\
\hline $\mathrm{H} 1$ & $1.13 \mathrm{E}-03$ & $1.13 \mathrm{E}-03$ & $1.13 \mathrm{E}-03$ & $1.13 \mathrm{E}-03$ & $1.13 \mathrm{E}-03$ & $1.13 \mathrm{E}-03$ & $1.13 \mathrm{E}-03$ & $1.13 \mathrm{E}-03$ & $1.13 \mathrm{E}-03$ \\
\hline $\mathrm{H} 2$ & $5.73 \mathrm{E}-07$ & $5.73 \mathrm{E}-07$ & $5.73 \mathrm{E}-07$ & $5.73 \mathrm{E}-07$ & $5.73 \mathrm{E}-07$ & $5.73 \mathrm{E}-07$ & $5.73 \mathrm{E}-07$ & $5.73 \mathrm{E}-07$ & $5.73 \mathrm{E}-07$ \\
\hline $\mathrm{H} 3$ & $1.07 \mathrm{E}-08$ & $1.07 \mathrm{E}-08$ & $1.07 \mathrm{E}-08$ & $1.07 \mathrm{E}-08$ & $1.07 \mathrm{E}-08$ & $1.06 \mathrm{E}-08$ & $1.05 \mathrm{E}-08$ & $1.04 \mathrm{E}-08$ & $1.02 \mathrm{E}-08$ \\
\hline HE 3 & $4.55 \mathrm{E}-11$ & $4.71 \mathrm{E}-11$ & $4.87 \mathrm{E}-11$ & $6.19 \mathrm{E}-11$ & $9.48 \mathrm{E}-11$ & $1.93 \mathrm{E}-10$ & $2.58 \mathrm{E}-10$ & $3.38 \mathrm{E}-10$ & $6.23 \mathrm{E}-10$ \\
\hline $\mathrm{HE} 4$ & $1.33 \mathrm{E}-02$ & $1.33 \mathrm{E}-02$ & $1.33 \mathrm{E}-02$ & $1.33 \mathrm{E}-02$ & $1.33 \mathrm{E}-02$ & $1.33 \mathrm{E}-02$ & $1.33 \mathrm{E}-02$ & $1.33 \mathrm{E}-02$ & $1.33 \mathrm{E}-02$ \\
\hline HE 6 & $9.05 \mathrm{E}-17$ & $0.00 \mathrm{E}+00$ & $0.00 \mathrm{E}+00$ & $0.00 \mathrm{E}+00$ & $0.00 \mathrm{E}+00$ & $0.00 \mathrm{E}+00$ & $0.00 \mathrm{E}+00$ & $0.00 \mathrm{E}+00$ & $0.00 \mathrm{E}+00$ \\
\hline LI 6 & $2.75 \mathrm{E}-10$ & $2.75 \mathrm{E}-10$ & $2.75 \mathrm{E}-10$ & $2.75 \mathrm{E}-10$ & $2.75 \mathrm{E}-10$ & $2.75 \mathrm{E}-10$ & $2.75 \mathrm{E}-10$ & $2.75 \mathrm{E}-10$ & $2.75 \mathrm{E}-10$ \\
\hline LI 7 & $2.89 \mathrm{E}-14$ & $2.89 \mathrm{E}-14$ & $2.89 \mathrm{E}-14$ & $2.89 \mathrm{E}-14$ & $2.89 \mathrm{E}-14$ & $2.89 \mathrm{E}-14$ & $2.89 \mathrm{E}-14$ & $2.89 \mathrm{E}-14$ & $2.89 \mathrm{E}-14$ \\
\hline BE 9 & $3.24 \mathrm{E}-06$ & $3.24 \mathrm{E}-06$ & $3.24 \mathrm{E}-06$ & $3.24 \mathrm{E}-06$ & $3.24 \mathrm{E}-06$ & $3.24 \mathrm{E}-06$ & $3.24 \mathrm{E}-06$ & $3.24 \mathrm{E}-06$ & $3.24 \mathrm{E}-06$ \\
\hline BE 10 & 8.29E-08 & 8.29E-08 & 8.29E-08 & 8.29E-08 & 8.29E-08 & 8.29E-08 & 8.29E-08 & 8.29E-08 & 8.29E-08 \\
\hline B 10 & $1.09 \mathrm{E}-14$ & $1.10 \mathrm{E}-14$ & $1.11 \mathrm{E}-14$ & $1.19 \mathrm{E}-14$ & $1.39 \mathrm{E}-14$ & $1.98 \mathrm{E}-14$ & $2.37 \mathrm{E}-14$ & $2.86 \mathrm{E}-14$ & 4.63E-14 \\
\hline
\end{tabular}


BSU-8242 3 DPA As-Run Physics Analysis

\begin{tabular}{|c|c|c|c|c|c|c|c|c|c|}
\hline \multicolumn{10}{|c|}{ ACTIVATION PRODUCTS (grams) } \\
\hline ISOTOPE & EOC & 1 Day & 2 Days & 10 Days & 30 Days & 90 Days & 130 Days & 180 Days & 360 Days \\
\hline B 11 & $5.46 \mathrm{E}-06$ & $5.46 \mathrm{E}-06$ & $5.46 \mathrm{E}-06$ & $5.46 \mathrm{E}-06$ & $5.46 \mathrm{E}-06$ & $5.46 \mathrm{E}-06$ & $5.46 \mathrm{E}-06$ & $5.46 \mathrm{E}-06$ & $5.46 \mathrm{E}-06$ \\
\hline $\mathrm{C} 12$ & $7.60 \mathrm{E}-01$ & $7.60 \mathrm{E}-01$ & $7.60 \mathrm{E}-01$ & $7.60 \mathrm{E}-01$ & $7.60 \mathrm{E}-01$ & $7.60 \mathrm{E}-01$ & 7.60E-01 & $7.60 \mathrm{E}-01$ & $7.60 \mathrm{E}-01$ \\
\hline C 13 & $9.25 \mathrm{E}-03$ & $9.25 \mathrm{E}-03$ & $9.25 \mathrm{E}-03$ & $9.25 \mathrm{E}-03$ & $9.25 \mathrm{E}-03$ & $9.25 \mathrm{E}-03$ & $9.25 \mathrm{E}-03$ & $9.25 \mathrm{E}-03$ & $9.25 \mathrm{E}-03$ \\
\hline $\mathrm{C} 14$ & $4.80 \mathrm{E}-05$ & 4.79E-05 & $4.79 \mathrm{E}-05$ & 4.79E-05 & 4.79E-05 & 4.79E-05 & 4.79E-05 & 4.79E-05 & 4.79E-05 \\
\hline $\mathrm{N} 14$ & $1.81 \mathrm{E}-02$ & $1.81 \mathrm{E}-02$ & $1.81 \mathrm{E}-02$ & $1.81 \mathrm{E}-02$ & $1.81 \mathrm{E}-02$ & $1.81 \mathrm{E}-02$ & $1.81 \mathrm{E}-02$ & $1.81 \mathrm{E}-02$ & $1.81 \mathrm{E}-02$ \\
\hline $\mathrm{N} 15$ & $7.33 \mathrm{E}-05$ & $7.33 \mathrm{E}-05$ & $7.33 \mathrm{E}-05$ & 7.33E-05 & 7.33E-05 & $7.33 \mathrm{E}-05$ & 7.33E-05 & $7.33 \mathrm{E}-05$ & $7.33 \mathrm{E}-05$ \\
\hline O 16 & $2.49 \mathrm{E}-12$ & $2.49 \mathrm{E}-12$ & $2.49 \mathrm{E}-12$ & $2.49 \mathrm{E}-12$ & $2.49 \mathrm{E}-12$ & $2.49 \mathrm{E}-12$ & $2.49 \mathrm{E}-12$ & $2.49 \mathrm{E}-12$ & $2.49 \mathrm{E}-12$ \\
\hline O 17 & $3.78 \mathrm{E}-17$ & $3.78 \mathrm{E}-17$ & $3.78 \mathrm{E}-17$ & $3.78 \mathrm{E}-17$ & $3.78 \mathrm{E}-17$ & $3.78 \mathrm{E}-17$ & $3.78 \mathrm{E}-17$ & $3.78 \mathrm{E}-17$ & $3.78 \mathrm{E}-17$ \\
\hline O 18 & $3.82 \mathrm{E}-09$ & $3.82 \mathrm{E}-09$ & $3.82 \mathrm{E}-09$ & $3.82 \mathrm{E}-09$ & $3.82 \mathrm{E}-09$ & $3.82 \mathrm{E}-09$ & $3.82 \mathrm{E}-09$ & $3.82 \mathrm{E}-09$ & $3.82 \mathrm{E}-09$ \\
\hline F 19 & $6.47 \mathrm{E}-16$ & $6.47 \mathrm{E}-16$ & $6.47 \mathrm{E}-16$ & $6.47 \mathrm{E}-16$ & $6.47 \mathrm{E}-16$ & $6.47 \mathrm{E}-16$ & $6.47 \mathrm{E}-16$ & $6.47 \mathrm{E}-16$ & $6.47 \mathrm{E}-16$ \\
\hline NE 20 & $1.48 \mathrm{E}-12$ & $1.48 \mathrm{E}-12$ & $1.48 \mathrm{E}-12$ & $1.48 \mathrm{E}-12$ & $1.48 \mathrm{E}-12$ & $1.48 \mathrm{E}-12$ & $1.48 \mathrm{E}-12$ & $1.48 \mathrm{E}-12$ & $1.48 \mathrm{E}-12$ \\
\hline NE 21 & $4.42 \mathrm{E}-06$ & $4.42 \mathrm{E}-06$ & $4.42 \mathrm{E}-06$ & 4.42E-06 & 4.42E-06 & $4.42 \mathrm{E}-06$ & 4.42E-06 & 4.42E-06 & 4.42E-06 \\
\hline NE 22 & $6.65 \mathrm{E}-06$ & $6.65 \mathrm{E}-06$ & $6.65 \mathrm{E}-06$ & $6.65 \mathrm{E}-06$ & $6.65 \mathrm{E}-06$ & $6.65 \mathrm{E}-06$ & $6.65 \mathrm{E}-06$ & $6.65 \mathrm{E}-06$ & $6.65 \mathrm{E}-06$ \\
\hline NE 23 & $4.66 \mathrm{E}-12$ & $0.00 \mathrm{E}+00$ & $0.00 \mathrm{E}+00$ & $0.00 \mathrm{E}+00$ & $0.00 \mathrm{E}+00$ & $0.00 \mathrm{E}+00$ & $0.00 \mathrm{E}+00$ & $0.00 \mathrm{E}+00$ & $0.00 \mathrm{E}+00$ \\
\hline NA 23 & $1.35 \mathrm{E}-06$ & $1.35 \mathrm{E}-06$ & $1.35 \mathrm{E}-06$ & $1.35 \mathrm{E}-06$ & $1.35 \mathrm{E}-06$ & $1.35 \mathrm{E}-06$ & $1.35 \mathrm{E}-06$ & $1.35 \mathrm{E}-06$ & $1.35 \mathrm{E}-06$ \\
\hline NA 24 & $1.74 \mathrm{E}-06$ & $5.72 \mathrm{E}-07$ & $1.89 \mathrm{E}-07$ & $2.65 \mathrm{E}-11$ & $6.17 \mathrm{E}-21$ & $0.00 \mathrm{E}+00$ & $0.00 \mathrm{E}+00$ & $0.00 \mathrm{E}+00$ & $0.00 \mathrm{E}+00$ \\
\hline NA 25 & $4.89 \mathrm{E}-12$ & $0.00 \mathrm{E}+00$ & $0.00 \mathrm{E}+00$ & $0.00 \mathrm{E}+00$ & $0.00 \mathrm{E}+00$ & $0.00 \mathrm{E}+00$ & $0.00 \mathrm{E}+00$ & $0.00 \mathrm{E}+00$ & $0.00 \mathrm{E}+00$ \\
\hline MG 24 & $7.52 \mathrm{E}-01$ & $7.52 \mathrm{E}-01$ & $7.52 \mathrm{E}-01$ & $7.52 \mathrm{E}-01$ & $7.52 \mathrm{E}-01$ & $7.52 \mathrm{E}-01$ & $7.52 \mathrm{E}-01$ & $7.52 \mathrm{E}-01$ & $7.52 \mathrm{E}-01$ \\
\hline MG 25 & $9.91 \mathrm{E}-02$ & $9.91 \mathrm{E}-02$ & $9.91 \mathrm{E}-02$ & $9.91 \mathrm{E}-02$ & $9.91 \mathrm{E}-02$ & $9.91 \mathrm{E}-02$ & $9.91 \mathrm{E}-02$ & $9.91 \mathrm{E}-02$ & $9.91 \mathrm{E}-02$ \\
\hline MG 26 & $1.14 \mathrm{E}-01$ & $1.14 \mathrm{E}-01$ & $1.14 \mathrm{E}-01$ & $1.14 \mathrm{E}-01$ & $1.14 \mathrm{E}-01$ & $1.14 \mathrm{E}-01$ & $1.14 \mathrm{E}-01$ & $1.14 \mathrm{E}-01$ & $1.14 \mathrm{E}-01$ \\
\hline MG 27 & $9.72 \mathrm{E}-08$ & $0.00 \mathrm{E}+00$ & $0.00 \mathrm{E}+00$ & $0.00 \mathrm{E}+00$ & $0.00 \mathrm{E}+00$ & $0.00 \mathrm{E}+00$ & $0.00 \mathrm{E}+00$ & $0.00 \mathrm{E}+00$ & $0.00 \mathrm{E}+00$ \\
\hline MG 28 & $3.78 \mathrm{E}-14$ & $1.71 \mathrm{E}-14$ & $7.71 \mathrm{E}-15$ & $1.33 \mathrm{E}-17$ & $1.63 \mathrm{E}-24$ & $0.00 \mathrm{E}+00$ & $0.00 \mathrm{E}+00$ & $0.00 \mathrm{E}+00$ & $0.00 \mathrm{E}+00$ \\
\hline AL 27 & $1.33 \mathrm{E}+02$ & $1.33 \mathrm{E}+02$ & $1.33 \mathrm{E}+02$ & $1.33 \mathrm{E}+02$ & $1.33 \mathrm{E}+02$ & $1.33 \mathrm{E}+02$ & $1.33 \mathrm{E}+02$ & $1.33 \mathrm{E}+02$ & $1.33 \mathrm{E}+02$ \\
\hline AL 28 & $5.71 \mathrm{E}-07$ & $3.05 \mathrm{E}-17$ & $1.38 \mathrm{E}-17$ & $2.38 \mathrm{E}-20$ & $2.92 \mathrm{E}-27$ & $0.00 \mathrm{E}+00$ & $0.00 \mathrm{E}+00$ & $0.00 \mathrm{E}+00$ & $0.00 \mathrm{E}+00$ \\
\hline AL 29 & $3.52 \mathrm{E}-11$ & $0.00 \mathrm{E}+00$ & $0.00 \mathrm{E}+00$ & $0.00 \mathrm{E}+00$ & $0.00 \mathrm{E}+00$ & $0.00 \mathrm{E}+00$ & $0.00 \mathrm{E}+00$ & $0.00 \mathrm{E}+00$ & $0.00 \mathrm{E}+00$ \\
\hline AL 30 & $3.32 \mathrm{E}-16$ & $0.00 \mathrm{E}+00$ & $0.00 \mathrm{E}+00$ & $0.00 \mathrm{E}+00$ & $0.00 \mathrm{E}+00$ & $0.00 \mathrm{E}+00$ & $0.00 \mathrm{E}+00$ & $0.00 \mathrm{E}+00$ & $0.00 \mathrm{E}+00$ \\
\hline
\end{tabular}


BSU-8242 3 DPA As-Run Physics Analysis

\begin{tabular}{|c|c|c|c|c|c|c|c|c|c|}
\hline \multicolumn{10}{|c|}{ ACTIVATION PRODUCTS (grams) } \\
\hline ISOTOPE & EOC & 1 Day & 2 Days & 10 Days & 30 Days & 90 Days & 130 Days & 180 Days & 360 Days \\
\hline SI 28 & $1.57 \mathrm{E}+00$ & $1.57 \mathrm{E}+00$ & $1.57 \mathrm{E}+00$ & $1.57 \mathrm{E}+00$ & $1.57 \mathrm{E}+00$ & $1.57 \mathrm{E}+00$ & $1.57 \mathrm{E}+00$ & $1.57 \mathrm{E}+00$ & $1.57 \mathrm{E}+00$ \\
\hline SI 29 & $8.02 \mathrm{E}-02$ & $8.02 \mathrm{E}-02$ & $8.02 \mathrm{E}-02$ & $8.02 \mathrm{E}-02$ & $8.02 \mathrm{E}-02$ & $8.02 \mathrm{E}-02$ & $8.02 \mathrm{E}-02$ & $8.02 \mathrm{E}-02$ & $8.02 \mathrm{E}-02$ \\
\hline SI 30 & $5.48 \mathrm{E}-02$ & $5.48 \mathrm{E}-02$ & $5.48 \mathrm{E}-02$ & $5.48 \mathrm{E}-02$ & $5.48 \mathrm{E}-02$ & $5.48 \mathrm{E}-02$ & $5.48 \mathrm{E}-02$ & $5.48 \mathrm{E}-02$ & $5.48 \mathrm{E}-02$ \\
\hline SI 31 & $9.95 \mathrm{E}-09$ & $1.75 \mathrm{E}-11$ & $3.05 \mathrm{E}-14$ & $0.00 \mathrm{E}+00$ & $0.00 \mathrm{E}+00$ & $0.00 \mathrm{E}+00$ & $0.00 \mathrm{E}+00$ & $0.00 \mathrm{E}+00$ & $0.00 \mathrm{E}+00$ \\
\hline SI 32 & $6.36 \mathrm{E}-12$ & $6.36 \mathrm{E}-12$ & $6.36 \mathrm{E}-12$ & $6.36 \mathrm{E}-12$ & $6.36 \mathrm{E}-12$ & $6.36 \mathrm{E}-12$ & $6.36 \mathrm{E}-12$ & $6.36 \mathrm{E}-12$ & $6.35 \mathrm{E}-12$ \\
\hline P 31 & $1.47 \mathrm{E}-02$ & $1.47 \mathrm{E}-02$ & $1.47 \mathrm{E}-02$ & $1.47 \mathrm{E}-02$ & $1.47 \mathrm{E}-02$ & $1.47 \mathrm{E}-02$ & $1.47 \mathrm{E}-02$ & $1.47 \mathrm{E}-02$ & $1.47 \mathrm{E}-02$ \\
\hline P 32 & $1.00 \mathrm{E}-06$ & $9.54 \mathrm{E}-07$ & $9.08 \mathrm{E}-07$ & $6.16 \mathrm{E}-07$ & $2.34 \mathrm{E}-07$ & $1.28 \mathrm{E}-08$ & $1.84 \mathrm{E}-09$ & $1.63 \mathrm{E}-10$ & $2.68 \mathrm{E}-14$ \\
\hline P 33 & $8.40 \mathrm{E}-11$ & $8.17 \mathrm{E}-11$ & $7.94 \mathrm{E}-11$ & $6.36 \mathrm{E}-11$ & $3.65 \mathrm{E}-11$ & $6.92 \mathrm{E}-12$ & $2.28 \mathrm{E}-12$ & $5.71 \mathrm{E}-13$ & $3.88 \mathrm{E}-15$ \\
\hline P 34 & $3.10 \mathrm{E}-15$ & $0.00 \mathrm{E}+00$ & $0.00 \mathrm{E}+00$ & $0.00 \mathrm{E}+00$ & $0.00 \mathrm{E}+00$ & $0.00 \mathrm{E}+00$ & $0.00 \mathrm{E}+00$ & $0.00 \mathrm{E}+00$ & $0.00 \mathrm{E}+00$ \\
\hline S 32 & $2.03 \mathrm{E}-02$ & $2.03 \mathrm{E}-02$ & $2.03 \mathrm{E}-02$ & $2.03 \mathrm{E}-02$ & $2.03 \mathrm{E}-02$ & $2.03 \mathrm{E}-02$ & $2.03 \mathrm{E}-02$ & $2.03 \mathrm{E}-02$ & $2.03 \mathrm{E}-02$ \\
\hline S 33 & $1.81 \mathrm{E}-04$ & $1.81 \mathrm{E}-04$ & $1.81 \mathrm{E}-04$ & $1.81 \mathrm{E}-04$ & $1.81 \mathrm{E}-04$ & $1.81 \mathrm{E}-04$ & $1.81 \mathrm{E}-04$ & $1.81 \mathrm{E}-04$ & $1.81 \mathrm{E}-04$ \\
\hline S 34 & $9.58 \mathrm{E}-04$ & $9.58 \mathrm{E}-04$ & $9.58 \mathrm{E}-04$ & $9.58 \mathrm{E}-04$ & $9.58 \mathrm{E}-04$ & $9.58 \mathrm{E}-04$ & $9.58 \mathrm{E}-04$ & $9.58 \mathrm{E}-04$ & $9.58 \mathrm{E}-04$ \\
\hline S 35 & $1.32 \mathrm{E}-07$ & $1.31 \mathrm{E}-07$ & $1.30 \mathrm{E}-07$ & $1.22 \mathrm{E}-07$ & $1.04 \mathrm{E}-07$ & $6.48 \mathrm{E}-08$ & $4.73 \mathrm{E}-08$ & $3.19 \mathrm{E}-08$ & $7.73 \mathrm{E}-09$ \\
\hline S 36 & $4.09 \mathrm{E}-06$ & $4.09 \mathrm{E}-06$ & $4.09 \mathrm{E}-06$ & $4.09 \mathrm{E}-06$ & $4.09 \mathrm{E}-06$ & $4.09 \mathrm{E}-06$ & $4.09 \mathrm{E}-06$ & $4.09 \mathrm{E}-06$ & $4.09 \mathrm{E}-06$ \\
\hline S 37 & $2.39 \mathrm{E}-14$ & $0.00 \mathrm{E}+00$ & $0.00 \mathrm{E}+00$ & $0.00 \mathrm{E}+00$ & $0.00 \mathrm{E}+00$ & $0.00 \mathrm{E}+00$ & $0.00 \mathrm{E}+00$ & $0.00 \mathrm{E}+00$ & $0.00 \mathrm{E}+00$ \\
\hline CL 35 & $1.82 \mathrm{E}-07$ & $1.83 \mathrm{E}-07$ & $1.84 \mathrm{E}-07$ & $1.92 \mathrm{E}-07$ & $2.09 \mathrm{E}-07$ & $2.49 \mathrm{E}-07$ & $2.66 \mathrm{E}-07$ & $2.81 \mathrm{E}-07$ & $3.06 \mathrm{E}-07$ \\
\hline CL 36 & $5.10 \mathrm{E}-09$ & $5.10 \mathrm{E}-09$ & $5.10 \mathrm{E}-09$ & $5.10 \mathrm{E}-09$ & $5.10 \mathrm{E}-09$ & $5.10 \mathrm{E}-09$ & $5.10 \mathrm{E}-09$ & $5.10 \mathrm{E}-09$ & $5.10 \mathrm{E}-09$ \\
\hline CL 37 & $8.70 \mathrm{E}-10$ & $8.70 \mathrm{E}-10$ & $8.70 \mathrm{E}-10$ & $8.70 \mathrm{E}-10$ & $8.70 \mathrm{E}-10$ & $8.70 \mathrm{E}-10$ & $8.70 \mathrm{E}-10$ & $8.70 \mathrm{E}-10$ & $8.70 \mathrm{E}-10$ \\
\hline CL 38 & $1.14 \mathrm{E}-16$ & $2.57 \mathrm{E}-28$ & $5.79 \mathrm{E}-40$ & $0.00 \mathrm{E}+00$ & $0.00 \mathrm{E}+00$ & $0.00 \mathrm{E}+00$ & $0.00 \mathrm{E}+00$ & $0.00 \mathrm{E}+00$ & $0.00 \mathrm{E}+00$ \\
\hline AR 36 & $2.31 \mathrm{E}-15$ & $2.35 \mathrm{E}-15$ & $2.38 \mathrm{E}-15$ & $2.63 \mathrm{E}-15$ & $3.26 \mathrm{E}-15$ & $5.15 \mathrm{E}-15$ & $6.42 \mathrm{E}-15$ & $7.99 \mathrm{E}-15$ & $1.37 \mathrm{E}-14$ \\
\hline AR 38 & $2.72 \mathrm{E}-13$ & $2.72 \mathrm{E}-13$ & $2.72 \mathrm{E}-13$ & $2.72 \mathrm{E}-13$ & $2.72 \mathrm{E}-13$ & $2.72 \mathrm{E}-13$ & $2.72 \mathrm{E}-13$ & $2.72 \mathrm{E}-13$ & $2.72 \mathrm{E}-13$ \\
\hline AR 39 & $8.36 \mathrm{E}-17$ & $8.36 \mathrm{E}-17$ & $8.36 \mathrm{E}-17$ & $8.35 \mathrm{E}-17$ & $8.35 \mathrm{E}-17$ & $8.35 \mathrm{E}-17$ & $8.35 \mathrm{E}-17$ & $8.34 \mathrm{E}-17$ & $8.33 \mathrm{E}-17$ \\
\hline AR 40 & $1.48 \mathrm{E}-12$ & $1.48 \mathrm{E}-12$ & $1.48 \mathrm{E}-12$ & $1.48 \mathrm{E}-12$ & $1.48 \mathrm{E}-12$ & $1.48 \mathrm{E}-12$ & $1.48 \mathrm{E}-12$ & $1.48 \mathrm{E}-12$ & $1.48 \mathrm{E}-12$ \\
\hline AR 41 & $5.90 \mathrm{E}-17$ & $6.55 \mathrm{E}-21$ & $7.27 \mathrm{E}-25$ & $0.00 \mathrm{E}+00$ & $0.00 \mathrm{E}+00$ & $0.00 \mathrm{E}+00$ & $0.00 \mathrm{E}+00$ & $0.00 \mathrm{E}+00$ & $0.00 \mathrm{E}+00$ \\
\hline K 41 & $4.81 \mathrm{E}-14$ & $4.82 \mathrm{E}-14$ & $4.82 \mathrm{E}-14$ & $4.82 \mathrm{E}-14$ & $4.82 \mathrm{E}-14$ & $4.82 \mathrm{E}-14$ & $4.82 \mathrm{E}-14$ & $4.82 \mathrm{E}-14$ & $4.82 \mathrm{E}-14$ \\
\hline K 43 & $9.70 \mathrm{E}-15$ & $4.65 \mathrm{E}-15$ & $2.23 \mathrm{E}-15$ & $6.16 \mathrm{E}-18$ & $2.49 \mathrm{E}-24$ & $1.81 \mathrm{E}-43$ & $0.00 \mathrm{E}+00$ & $0.00 \mathrm{E}+00$ & $0.00 \mathrm{E}+00$ \\
\hline
\end{tabular}


BSU-8242 3 DPA As-Run Physics Analysis

\begin{tabular}{|c|c|c|c|c|c|c|c|c|c|}
\hline \multicolumn{10}{|c|}{ ACTIVATION PRODUCTS (grams) } \\
\hline ISOTOPE & EOC & 1 Day & 2 Days & 10 Days & 30 Days & 90 Days & 130 Days & 180 Days & 360 Days \\
\hline K 44 & $2.86 \mathrm{E}-17$ & $5.66 \mathrm{E}-37$ & $0.00 \mathrm{E}+00$ & $0.00 \mathrm{E}+00$ & $0.00 \mathrm{E}+00$ & $0.00 \mathrm{E}+00$ & $0.00 \mathrm{E}+00$ & $0.00 \mathrm{E}+00$ & $0.00 \mathrm{E}+00$ \\
\hline CA 42 & $4.62 \mathrm{E}-16$ & $4.66 \mathrm{E}-16$ & $4.66 \mathrm{E}-16$ & $4.67 \mathrm{E}-16$ & $4.67 \mathrm{E}-16$ & 4.67E-16 & 4.67E-16 & 4.67E-16 & 4.67E-16 \\
\hline CA 43 & $8.46 \mathrm{E}-08$ & $8.46 \mathrm{E}-08$ & $8.46 \mathrm{E}-08$ & $8.46 \mathrm{E}-08$ & $8.46 \mathrm{E}-08$ & $8.46 \mathrm{E}-08$ & $8.46 \mathrm{E}-08$ & $8.46 \mathrm{E}-08$ & $8.46 \mathrm{E}-08$ \\
\hline CA 44 & $6.53 \mathrm{E}-07$ & $6.53 \mathrm{E}-07$ & $6.53 \mathrm{E}-07$ & $6.53 \mathrm{E}-07$ & $6.53 \mathrm{E}-07$ & $6.53 \mathrm{E}-07$ & $6.53 \mathrm{E}-07$ & $6.53 \mathrm{E}-07$ & $6.53 \mathrm{E}-07$ \\
\hline CA 45 & 7.77E-09 & $7.73 \mathrm{E}-09$ & 7.70E-09 & 7.44E-09 & $6.84 \mathrm{E}-09$ & $5.30 \mathrm{E}-09$ & 4.47E-09 & $3.61 \mathrm{E}-09$ & $1.68 \mathrm{E}-09$ \\
\hline CA 46 & 7.79E-08 & 7.79E-08 & 7.79E-08 & 7.79E-08 & 7.79E-08 & $7.79 \mathrm{E}-08$ & 7.79E-08 & 7.79E-08 & 7.79E-08 \\
\hline CA 47 & $4.04 \mathrm{E}-12$ & $3.47 \mathrm{E}-12$ & $2.97 \mathrm{E}-12$ & $8.76 \mathrm{E}-13$ & $4.12 \mathrm{E}-14$ & $4.30 \mathrm{E}-18$ & $9.53 \mathrm{E}-21$ & $4.58 \mathrm{E}-24$ & $5.18 \mathrm{E}-36$ \\
\hline $\mathrm{SC} 45$ & $5.81 \mathrm{E}-09$ & $5.84 \mathrm{E}-09$ & $5.87 \mathrm{E}-09$ & $6.13 \mathrm{E}-09$ & $6.74 \mathrm{E}-09$ & 8.28E-09 & $9.11 \mathrm{E}-09$ & $9.96 \mathrm{E}-09$ & $1.19 \mathrm{E}-08$ \\
\hline $\mathrm{SC} 46$ & $7.08 \mathrm{E}-07$ & $7.02 \mathrm{E}-07$ & $6.96 \mathrm{E}-07$ & $6.52 \mathrm{E}-07$ & $5.52 \mathrm{E}-07$ & $3.36 \mathrm{E}-07$ & $2.42 \mathrm{E}-07$ & $1.60 \mathrm{E}-07$ & $3.60 \mathrm{E}-08$ \\
\hline $\mathrm{SC} 46 \mathrm{M}$ & $1.32 \mathrm{E}-16$ & $0.00 \mathrm{E}+00$ & $0.00 \mathrm{E}+00$ & $0.00 \mathrm{E}+00$ & $0.00 \mathrm{E}+00$ & $0.00 \mathrm{E}+00$ & $0.00 \mathrm{E}+00$ & $0.00 \mathrm{E}+00$ & $0.00 \mathrm{E}+00$ \\
\hline $\mathrm{SC} 47$ & $7.50 \mathrm{E}-08$ & $6.10 \mathrm{E}-08$ & $4.96 \mathrm{E}-08$ & $9.48 \mathrm{E}-09$ & $1.51 \mathrm{E}-10$ & $6.28 \mathrm{E}-16$ & $1.87 \mathrm{E}-19$ & $1.81 \mathrm{E}-23$ & $1.46 \mathrm{E}-35$ \\
\hline $\mathrm{SC} 48$ & 3.94E-09 & $2.70 \mathrm{E}-09$ & $1.84 \mathrm{E}-09$ & $8.83 \mathrm{E}-11$ & 4.44E-14 & $5.65 \mathrm{E}-24$ & $1.43 \mathrm{E}-30$ & $8.10 \mathrm{E}-39$ & $0.00 \mathrm{E}+00$ \\
\hline $\mathrm{SC} 49$ & $8.05 \mathrm{E}-11$ & $2.33 \mathrm{E}-18$ & $6.73 \mathrm{E}-26$ & $0.00 \mathrm{E}+00$ & $0.00 \mathrm{E}+00$ & $0.00 \mathrm{E}+00$ & $0.00 \mathrm{E}+00$ & $0.00 \mathrm{E}+00$ & $0.00 \mathrm{E}+00$ \\
\hline SC 50 & $4.52 \mathrm{E}-14$ & $0.00 \mathrm{E}+00$ & $0.00 \mathrm{E}+00$ & $0.00 \mathrm{E}+00$ & $0.00 \mathrm{E}+00$ & $0.00 \mathrm{E}+00$ & $0.00 \mathrm{E}+00$ & $0.00 \mathrm{E}+00$ & $0.00 \mathrm{E}+00$ \\
\hline TI 46 & $3.07 \mathrm{E}-02$ & $3.07 \mathrm{E}-02$ & $3.07 \mathrm{E}-02$ & $3.07 \mathrm{E}-02$ & $3.07 \mathrm{E}-02$ & $3.07 \mathrm{E}-02$ & $3.07 \mathrm{E}-02$ & $3.07 \mathrm{E}-02$ & $3.07 \mathrm{E}-02$ \\
\hline TI 47 & $2.83 \mathrm{E}-02$ & $2.83 \mathrm{E}-02$ & $2.83 \mathrm{E}-02$ & $2.83 \mathrm{E}-02$ & $2.83 \mathrm{E}-02$ & $2.83 \mathrm{E}-02$ & $2.83 \mathrm{E}-02$ & $2.83 \mathrm{E}-02$ & $2.83 \mathrm{E}-02$ \\
\hline TI 48 & $2.83 \mathrm{E}-01$ & $2.83 \mathrm{E}-01$ & $2.83 \mathrm{E}-01$ & $2.83 \mathrm{E}-01$ & $2.83 \mathrm{E}-01$ & $2.83 \mathrm{E}-01$ & $2.83 \mathrm{E}-01$ & $2.83 \mathrm{E}-01$ & $2.83 \mathrm{E}-01$ \\
\hline TI 49 & $2.44 \mathrm{E}-02$ & $2.44 \mathrm{E}-02$ & $2.44 \mathrm{E}-02$ & $2.44 \mathrm{E}-02$ & $2.44 \mathrm{E}-02$ & $2.44 \mathrm{E}-02$ & $2.44 \mathrm{E}-02$ & $2.44 \mathrm{E}-02$ & $2.44 \mathrm{E}-02$ \\
\hline TI 50 & $2.13 \mathrm{E}-02$ & $2.13 \mathrm{E}-02$ & $2.13 \mathrm{E}-02$ & $2.13 \mathrm{E}-02$ & $2.13 \mathrm{E}-02$ & $2.13 \mathrm{E}-02$ & $2.13 \mathrm{E}-02$ & $2.13 \mathrm{E}-02$ & $2.13 \mathrm{E}-02$ \\
\hline TI 51 & $2.04 \mathrm{E}-10$ & $0.00 \mathrm{E}+00$ & $0.00 \mathrm{E}+00$ & $0.00 \mathrm{E}+00$ & $0.00 \mathrm{E}+00$ & $0.00 \mathrm{E}+00$ & $0.00 \mathrm{E}+00$ & $0.00 \mathrm{E}+00$ & $0.00 \mathrm{E}+00$ \\
\hline V 50 & $9.09 \mathrm{E}-04$ & $9.09 \mathrm{E}-04$ & $9.09 \mathrm{E}-04$ & $9.09 \mathrm{E}-04$ & $9.09 \mathrm{E}-04$ & $9.09 \mathrm{E}-04$ & $9.09 \mathrm{E}-04$ & $9.09 \mathrm{E}-04$ & $9.09 \mathrm{E}-04$ \\
\hline V 51 & $2.27 \mathrm{E}-01$ & $2.27 \mathrm{E}-01$ & $2.28 \mathrm{E}-01$ & $2.32 \mathrm{E}-01$ & $2.40 \mathrm{E}-01$ & $2.49 \mathrm{E}-01$ & $2.50 \mathrm{E}-01$ & $2.51 \mathrm{E}-01$ & $2.51 \mathrm{E}-01$ \\
\hline V 52 & $4.97 \mathrm{E}-08$ & $0.00 \mathrm{E}+00$ & $0.00 \mathrm{E}+00$ & $0.00 \mathrm{E}+00$ & $0.00 \mathrm{E}+00$ & $0.00 \mathrm{E}+00$ & $0.00 \mathrm{E}+00$ & $0.00 \mathrm{E}+00$ & $0.00 \mathrm{E}+00$ \\
\hline V 53 & $5.64 \mathrm{E}-11$ & $0.00 \mathrm{E}+00$ & $0.00 \mathrm{E}+00$ & $0.00 \mathrm{E}+00$ & $0.00 \mathrm{E}+00$ & $0.00 \mathrm{E}+00$ & $0.00 \mathrm{E}+00$ & $0.00 \mathrm{E}+00$ & $0.00 \mathrm{E}+00$ \\
\hline V 54 & $3.54 \mathrm{E}-13$ & $0.00 \mathrm{E}+00$ & $0.00 \mathrm{E}+00$ & $0.00 \mathrm{E}+00$ & $0.00 \mathrm{E}+00$ & $0.00 \mathrm{E}+00$ & $0.00 \mathrm{E}+00$ & $0.00 \mathrm{E}+00$ & $0.00 \mathrm{E}+00$ \\
\hline CR 50 & $5.89 \mathrm{E}+00$ & $5.89 \mathrm{E}+00$ & $5.89 \mathrm{E}+00$ & $5.89 \mathrm{E}+00$ & $5.89 \mathrm{E}+00$ & $5.89 \mathrm{E}+00$ & $5.89 \mathrm{E}+00$ & $5.89 \mathrm{E}+00$ & $5.89 \mathrm{E}+00$ \\
\hline
\end{tabular}


BSU-8242 3 DPA As-Run Physics Analysis

\begin{tabular}{|c|c|c|c|c|c|c|c|c|c|}
\hline \multicolumn{10}{|c|}{ ACTIVATION PRODUCTS (grams) } \\
\hline ISOTOPE & EOC & 1 Day & 2 Days & 10 Days & 30 Days & 90 Days & 130 Days & 180 Days & 360 Days \\
\hline CR 51 & $2.47 \mathrm{E}-02$ & $2.41 \mathrm{E}-02$ & $2.35 \mathrm{E}-02$ & $1.92 \mathrm{E}-02$ & $1.17 \mathrm{E}-02$ & $2.60 \mathrm{E}-03$ & $9.56 \mathrm{E}-04$ & $2.74 \mathrm{E}-04$ & $3.03 \mathrm{E}-06$ \\
\hline CR 52 & $1.20 \mathrm{E}+02$ & $1.20 \mathrm{E}+02$ & $1.20 \mathrm{E}+02$ & $1.20 \mathrm{E}+02$ & $1.20 \mathrm{E}+02$ & $1.20 \mathrm{E}+02$ & $1.20 \mathrm{E}+02$ & $1.20 \mathrm{E}+02$ & $1.20 \mathrm{E}+02$ \\
\hline CR 53 & $1.42 \mathrm{E}+01$ & $1.42 \mathrm{E}+01$ & $1.42 \mathrm{E}+01$ & $1.42 \mathrm{E}+01$ & $1.42 \mathrm{E}+01$ & $1.42 \mathrm{E}+01$ & $1.42 \mathrm{E}+01$ & $1.42 \mathrm{E}+01$ & $1.42 \mathrm{E}+01$ \\
\hline CR 54 & $3.88 \mathrm{E}+00$ & $3.88 \mathrm{E}+00$ & $3.88 \mathrm{E}+00$ & $3.88 \mathrm{E}+00$ & $3.88 \mathrm{E}+00$ & $3.88 \mathrm{E}+00$ & $3.88 \mathrm{E}+00$ & $3.88 \mathrm{E}+00$ & $3.88 \mathrm{E}+00$ \\
\hline CR 55 & $3.84 \mathrm{E}-08$ & $0.00 \mathrm{E}+00$ & $0.00 \mathrm{E}+00$ & $0.00 \mathrm{E}+00$ & $0.00 \mathrm{E}+00$ & $0.00 \mathrm{E}+00$ & $0.00 \mathrm{E}+00$ & $0.00 \mathrm{E}+00$ & $0.00 \mathrm{E}+00$ \\
\hline MN 54 & $4.09 \mathrm{E}-03$ & $4.08 \mathrm{E}-03$ & $4.07 \mathrm{E}-03$ & $4.00 \mathrm{E}-03$ & $3.83 \mathrm{E}-03$ & $3.35 \mathrm{E}-03$ & $3.07 \mathrm{E}-03$ & $2.74 \mathrm{E}-03$ & $1.84 \mathrm{E}-03$ \\
\hline MN 55 & $1.65 \mathrm{E}+00$ & $1.65 \mathrm{E}+00$ & $1.65 \mathrm{E}+00$ & $1.65 \mathrm{E}+00$ & $1.65 \mathrm{E}+00$ & $1.65 \mathrm{E}+00$ & $1.65 \mathrm{E}+00$ & $1.65 \mathrm{E}+00$ & $1.66 \mathrm{E}+00$ \\
\hline MN 56 & $4.00 \mathrm{E}-05$ & $6.31 \mathrm{E}-08$ & $9.96 \mathrm{E}-11$ & $0.00 \mathrm{E}+00$ & $0.00 \mathrm{E}+00$ & $0.00 \mathrm{E}+00$ & $0.00 \mathrm{E}+00$ & $0.00 \mathrm{E}+00$ & $0.00 \mathrm{E}+00$ \\
\hline MN 57 & $1.43 \mathrm{E}-10$ & $0.00 \mathrm{E}+00$ & $0.00 \mathrm{E}+00$ & $0.00 \mathrm{E}+00$ & $0.00 \mathrm{E}+00$ & $0.00 \mathrm{E}+00$ & $0.00 \mathrm{E}+00$ & $0.00 \mathrm{E}+00$ & $0.00 \mathrm{E}+00$ \\
\hline MN 58 & $2.95 \mathrm{E}-13$ & $0.00 \mathrm{E}+00$ & $0.00 \mathrm{E}+00$ & $0.00 \mathrm{E}+00$ & $0.00 \mathrm{E}+00$ & $0.00 \mathrm{E}+00$ & $0.00 \mathrm{E}+00$ & $0.00 \mathrm{E}+00$ & $0.00 \mathrm{E}+00$ \\
\hline FE 54 & $1.94 \mathrm{E}+01$ & $1.94 \mathrm{E}+01$ & $1.94 \mathrm{E}+01$ & $1.94 \mathrm{E}+01$ & $1.94 \mathrm{E}+01$ & $1.94 \mathrm{E}+01$ & $1.94 \mathrm{E}+01$ & $1.94 \mathrm{E}+01$ & $1.94 \mathrm{E}+01$ \\
\hline FE 55 & $5.67 \mathrm{E}-02$ & $5.66 \mathrm{E}-02$ & $5.66 \mathrm{E}-02$ & $5.63 \mathrm{E}-02$ & $5.55 \mathrm{E}-02$ & $5.31 \mathrm{E}-02$ & $5.16 \mathrm{E}-02$ & 4.97E-02 & 4.36E-02 \\
\hline FE 56 & $3.18 \mathrm{E}+02$ & $3.18 \mathrm{E}+02$ & $3.18 \mathrm{E}+02$ & $3.18 \mathrm{E}+02$ & $3.18 \mathrm{E}+02$ & $3.18 \mathrm{E}+02$ & $3.18 \mathrm{E}+02$ & $3.18 \mathrm{E}+02$ & $3.18 \mathrm{E}+02$ \\
\hline FE 57 & $8.85 \mathrm{E}+00$ & $8.85 \mathrm{E}+00$ & $8.85 \mathrm{E}+00$ & $8.85 \mathrm{E}+00$ & $8.85 \mathrm{E}+00$ & $8.85 \mathrm{E}+00$ & $8.85 \mathrm{E}+00$ & $8.85 \mathrm{E}+00$ & $8.85 \mathrm{E}+00$ \\
\hline FE 58 & $1.09 \mathrm{E}+00$ & $1.09 \mathrm{E}+00$ & $1.09 \mathrm{E}+00$ & $1.09 \mathrm{E}+00$ & $1.09 \mathrm{E}+00$ & $1.09 \mathrm{E}+00$ & $1.09 \mathrm{E}+00$ & $1.09 \mathrm{E}+00$ & $1.09 \mathrm{E}+00$ \\
\hline FE 59 & $5.34 \mathrm{E}-04$ & $5.26 \mathrm{E}-04$ & $5.18 \mathrm{E}-04$ & $4.58 \mathrm{E}-04$ & $3.36 \mathrm{E}-04$ & $1.34 \mathrm{E}-04$ & $7.21 \mathrm{E}-05$ & $3.34 \mathrm{E}-05$ & $2.09 \mathrm{E}-06$ \\
\hline CO 58 & $6.60 \mathrm{E}-03$ & $6.54 \mathrm{E}-03$ & $6.47 \mathrm{E}-03$ & $5.99 \mathrm{E}-03$ & $4.92 \mathrm{E}-03$ & $2.73 \mathrm{E}-03$ & $1.85 \mathrm{E}-03$ & $1.13 \mathrm{E}-03$ & $1.94 \mathrm{E}-04$ \\
\hline CO 59 & $1.47 \mathrm{E}+00$ & $1.47 \mathrm{E}+00$ & $1.47 \mathrm{E}+00$ & $1.47 \mathrm{E}+00$ & $1.47 \mathrm{E}+00$ & $1.47 \mathrm{E}+00$ & $1.47 \mathrm{E}+00$ & $1.47 \mathrm{E}+00$ & $1.47 \mathrm{E}+00$ \\
\hline $\mathrm{CO} 60$ & $1.02 \mathrm{E}-01$ & $1.02 \mathrm{E}-01$ & $1.02 \mathrm{E}-01$ & $1.02 \mathrm{E}-01$ & $1.01 \mathrm{E}-01$ & $9.92 \mathrm{E}-02$ & $9.78 \mathrm{E}-02$ & $9.60 \mathrm{E}-02$ & $9.00 \mathrm{E}-02$ \\
\hline $\mathrm{CO} 60 \mathrm{M}$ & $3.33 \mathrm{E}-06$ & $0.00 \mathrm{E}+00$ & $0.00 \mathrm{E}+00$ & $0.00 \mathrm{E}+00$ & $0.00 \mathrm{E}+00$ & $0.00 \mathrm{E}+00$ & $0.00 \mathrm{E}+00$ & $0.00 \mathrm{E}+00$ & $0.00 \mathrm{E}+00$ \\
\hline $\mathrm{CO} 61$ & $2.36 \mathrm{E}-07$ & $9.87 \mathrm{E}-12$ & $4.13 \mathrm{E}-16$ & $0.00 \mathrm{E}+00$ & $0.00 \mathrm{E}+00$ & $0.00 \mathrm{E}+00$ & $0.00 \mathrm{E}+00$ & $0.00 \mathrm{E}+00$ & $0.00 \mathrm{E}+00$ \\
\hline $\mathrm{CO} 62$ & $3.21 \mathrm{E}-11$ & $0.00 \mathrm{E}+00$ & $0.00 \mathrm{E}+00$ & $0.00 \mathrm{E}+00$ & $0.00 \mathrm{E}+00$ & $0.00 \mathrm{E}+00$ & $0.00 \mathrm{E}+00$ & $0.00 \mathrm{E}+00$ & $0.00 \mathrm{E}+00$ \\
\hline NI 58 & $1.45 \mathrm{E}+02$ & $1.45 \mathrm{E}+02$ & $1.45 \mathrm{E}+02$ & $1.45 \mathrm{E}+02$ & $1.45 \mathrm{E}+02$ & $1.45 \mathrm{E}+02$ & $1.45 \mathrm{E}+02$ & $1.45 \mathrm{E}+02$ & $1.45 \mathrm{E}+02$ \\
\hline NI 59 & $9.65 \mathrm{E}-01$ & $9.65 \mathrm{E}-01$ & $9.65 \mathrm{E}-01$ & $9.65 \mathrm{E}-01$ & $9.65 \mathrm{E}-01$ & $9.65 \mathrm{E}-01$ & $9.65 \mathrm{E}-01$ & $9.65 \mathrm{E}-01$ & $9.65 \mathrm{E}-01$ \\
\hline NI 60 & $5.75 \mathrm{E}+01$ & $5.75 \mathrm{E}+01$ & $5.75 \mathrm{E}+01$ & $5.75 \mathrm{E}+01$ & $5.75 \mathrm{E}+01$ & $5.75 \mathrm{E}+01$ & $5.76 \mathrm{E}+01$ & $5.76 \mathrm{E}+01$ & $5.76 \mathrm{E}+01$ \\
\hline NI 61 & $2.76 \mathrm{E}+00$ & $2.76 \mathrm{E}+00$ & $2.76 \mathrm{E}+00$ & $2.76 \mathrm{E}+00$ & $2.76 \mathrm{E}+00$ & $2.76 \mathrm{E}+00$ & $2.76 \mathrm{E}+00$ & $2.76 \mathrm{E}+00$ & $2.76 \mathrm{E}+00$ \\
\hline
\end{tabular}


BSU-8242 3 DPA As-Run Physics Analysis

\begin{tabular}{|c|c|c|c|c|c|c|c|c|c|}
\hline \multicolumn{10}{|c|}{ ACTIVATION PRODUCTS (grams) } \\
\hline ISOTOPE & EOC & 1 Day & 2 Days & 10 Days & 30 Days & 90 Days & 130 Days & 180 Days & 360 Days \\
\hline NI 62 & $8.05 \mathrm{E}+00$ & $8.05 \mathrm{E}+00$ & $8.05 \mathrm{E}+00$ & $8.05 \mathrm{E}+00$ & $8.05 \mathrm{E}+00$ & $8.05 \mathrm{E}+00$ & $8.05 \mathrm{E}+00$ & $8.05 \mathrm{E}+00$ & $8.05 \mathrm{E}+00$ \\
\hline NI 63 & $1.56 \mathrm{E}-01$ & $1.56 \mathrm{E}-01$ & $1.56 \mathrm{E}-01$ & $1.56 \mathrm{E}-01$ & $1.56 \mathrm{E}-01$ & $1.56 \mathrm{E}-01$ & $1.56 \mathrm{E}-01$ & $1.55 \mathrm{E}-01$ & $1.55 \mathrm{E}-01$ \\
\hline NI 64 & $2.15 \mathrm{E}+00$ & $2.15 \mathrm{E}+00$ & $2.15 \mathrm{E}+00$ & $2.15 \mathrm{E}+00$ & $2.15 \mathrm{E}+00$ & $2.15 \mathrm{E}+00$ & $2.15 \mathrm{E}+00$ & $2.15 \mathrm{E}+00$ & $2.15 \mathrm{E}+00$ \\
\hline NI 65 & $3.96 \mathrm{E}-06$ & $5.36 \mathrm{E}-09$ & $7.24 \mathrm{E}-12$ & $0.00 \mathrm{E}+00$ & $0.00 \mathrm{E}+00$ & $0.00 \mathrm{E}+00$ & $0.00 \mathrm{E}+00$ & $0.00 \mathrm{E}+00$ & $0.00 \mathrm{E}+00$ \\
\hline NI 66 & 2.39E-09 & $1.76 \mathrm{E}-09$ & $1.30 \mathrm{E}-09$ & $1.14 \mathrm{E}-10$ & $2.57 \mathrm{E}-13$ & $2.96 \mathrm{E}-21$ & $1.51 \mathrm{E}-26$ & $3.67 \mathrm{E}-33$ & $0.00 \mathrm{E}+00$ \\
\hline CU 63 & $7.98 \mathrm{E}-01$ & $7.98 \mathrm{E}-01$ & $7.98 \mathrm{E}-01$ & $7.98 \mathrm{E}-01$ & 7.99E-01 & 7.99E-01 & 7.99E-01 & 7.99E-01 & $8.00 \mathrm{E}-01$ \\
\hline CU 64 & $2.44 \mathrm{E}-05$ & $6.57 \mathrm{E}-06$ & $1.77 \mathrm{E}-06$ & $4.99 \mathrm{E}-11$ & $2.09 \mathrm{E}-22$ & $0.00 \mathrm{E}+00$ & $0.00 \mathrm{E}+00$ & $0.00 \mathrm{E}+00$ & $0.00 \mathrm{E}+00$ \\
\hline CU 65 & $3.72 \mathrm{E}-01$ & $3.72 \mathrm{E}-01$ & $3.72 \mathrm{E}-01$ & $3.72 \mathrm{E}-01$ & $3.72 \mathrm{E}-01$ & $3.72 \mathrm{E}-01$ & $3.72 \mathrm{E}-01$ & $3.72 \mathrm{E}-01$ & $3.72 \mathrm{E}-01$ \\
\hline CU 66 & $3.68 \mathrm{E}-08$ & $2.75 \mathrm{E}-12$ & $2.03 \mathrm{E}-12$ & $1.77 \mathrm{E}-13$ & $4.01 \mathrm{E}-16$ & 4.62E-24 & $2.36 \mathrm{E}-29$ & $5.73 \mathrm{E}-36$ & $0.00 \mathrm{E}+00$ \\
\hline CU 67 & $1.40 \mathrm{E}-10$ & $1.07 \mathrm{E}-10$ & $8.17 \mathrm{E}-11$ & $9.50 \mathrm{E}-12$ & $4.39 \mathrm{E}-14$ & $4.31 \mathrm{E}-21$ & $9.19 \mathrm{E}-26$ & $1.33 \mathrm{E}-31$ & $0.00 \mathrm{E}+00$ \\
\hline $\mathrm{ZN} 64$ & $2.14 \mathrm{E}-03$ & $2.14 \mathrm{E}-03$ & $2.14 \mathrm{E}-03$ & $2.14 \mathrm{E}-03$ & $2.14 \mathrm{E}-03$ & $2.14 \mathrm{E}-03$ & $2.14 \mathrm{E}-03$ & $2.14 \mathrm{E}-03$ & $2.14 \mathrm{E}-03$ \\
\hline $\mathrm{ZN} 65$ & $1.23 \mathrm{E}-06$ & $1.23 \mathrm{E}-06$ & $1.22 \mathrm{E}-06$ & $1.19 \mathrm{E}-06$ & $1.13 \mathrm{E}-06$ & $9.51 \mathrm{E}-07$ & $8.49 \mathrm{E}-07$ & $7.36 \mathrm{E}-07$ & 4.42E-07 \\
\hline $\mathrm{ZN} 66$ & $1.29 \mathrm{E}-03$ & $1.29 \mathrm{E}-03$ & $1.29 \mathrm{E}-03$ & $1.29 \mathrm{E}-03$ & $1.29 \mathrm{E}-03$ & $1.29 \mathrm{E}-03$ & $1.29 \mathrm{E}-03$ & $1.29 \mathrm{E}-03$ & $1.29 \mathrm{E}-03$ \\
\hline $\mathrm{ZN} 67$ & $8.48 \mathrm{E}-07$ & $8.48 \mathrm{E}-07$ & $8.48 \mathrm{E}-07$ & $8.48 \mathrm{E}-07$ & $8.48 \mathrm{E}-07$ & $8.48 \mathrm{E}-07$ & $8.48 \mathrm{E}-07$ & $8.48 \mathrm{E}-07$ & $8.48 \mathrm{E}-07$ \\
\hline $\mathrm{ZN} 68$ & 4.42E-09 & 4.42E-09 & 4.42E-09 & 4.42E-09 & 4.42E-09 & 4.42E-09 & 4.42E-09 & 4.42E-09 & 4.42E-09 \\
\hline ZN 69 & $3.49 \mathrm{E}-15$ & $7.51 \mathrm{E}-17$ & $2.24 \mathrm{E}-17$ & $1.42 \mathrm{E}-21$ & $4.47 \mathrm{E}-32$ & $0.00 \mathrm{E}+00$ & $0.00 \mathrm{E}+00$ & $0.00 \mathrm{E}+00$ & $0.00 \mathrm{E}+00$ \\
\hline $\mathrm{ZN} \mathrm{69M}$ & $3.40 \mathrm{E}-15$ & $1.01 \mathrm{E}-15$ & $3.03 \mathrm{E}-16$ & $1.91 \mathrm{E}-20$ & $6.03 \mathrm{E}-31$ & $0.00 \mathrm{E}+00$ & $0.00 \mathrm{E}+00$ & $0.00 \mathrm{E}+00$ & $0.00 \mathrm{E}+00$ \\
\hline GA 69 & $2.76 \mathrm{E}-12$ & $2.76 \mathrm{E}-12$ & $2.76 \mathrm{E}-12$ & $2.76 \mathrm{E}-12$ & $2.76 \mathrm{E}-12$ & $2.76 \mathrm{E}-12$ & $2.76 \mathrm{E}-12$ & $2.76 \mathrm{E}-12$ & $2.76 \mathrm{E}-12$ \\
\hline GE 70 & $4.26 \mathrm{E}-15$ & $4.27 \mathrm{E}-15$ & $4.27 \mathrm{E}-15$ & $4.27 \mathrm{E}-15$ & 4.27E-15 & 4.27E-15 & 4.27E-15 & 4.27E-15 & 4.27E-15 \\
\hline SR 87 & $6.89 \mathrm{E}-15$ & $6.89 \mathrm{E}-15$ & $6.89 \mathrm{E}-15$ & $6.89 \mathrm{E}-15$ & $6.89 \mathrm{E}-15$ & $6.89 \mathrm{E}-15$ & $6.89 \mathrm{E}-15$ & $6.89 \mathrm{E}-15$ & $6.89 \mathrm{E}-15$ \\
\hline SR 88 & $8.81 \mathrm{E}-12$ & $8.81 \mathrm{E}-12$ & $8.81 \mathrm{E}-12$ & $8.81 \mathrm{E}-12$ & $8.81 \mathrm{E}-12$ & $8.81 \mathrm{E}-12$ & $8.81 \mathrm{E}-12$ & $8.81 \mathrm{E}-12$ & $8.81 \mathrm{E}-12$ \\
\hline SR 89 & $4.61 \mathrm{E}-12$ & $4.55 \mathrm{E}-12$ & $4.49 \mathrm{E}-12$ & $4.02 \mathrm{E}-12$ & $3.06 \mathrm{E}-12$ & $1.34 \mathrm{E}-12$ & $7.75 \mathrm{E}-13$ & $3.90 \mathrm{E}-13$ & $3.30 \mathrm{E}-14$ \\
\hline SR 90 & $1.20 \mathrm{E}-12$ & $1.20 \mathrm{E}-12$ & $1.20 \mathrm{E}-12$ & $1.20 \mathrm{E}-12$ & $1.20 \mathrm{E}-12$ & $1.20 \mathrm{E}-12$ & $1.19 \mathrm{E}-12$ & $1.19 \mathrm{E}-12$ & $1.18 \mathrm{E}-12$ \\
\hline SR 91 & $1.12 \mathrm{E}-15$ & $1.94 \mathrm{E}-16$ & $3.37 \mathrm{E}-17$ & $2.78 \mathrm{E}-23$ & $1.72 \mathrm{E}-38$ & $0.00 \mathrm{E}+00$ & $0.00 \mathrm{E}+00$ & $0.00 \mathrm{E}+00$ & $0.00 \mathrm{E}+00$ \\
\hline Y 89 & $1.94 \mathrm{E}-07$ & $1.95 \mathrm{E}-07$ & $1.96 \mathrm{E}-07$ & $1.98 \mathrm{E}-07$ & $1.99 \mathrm{E}-07$ & $1.99 \mathrm{E}-07$ & $1.99 \mathrm{E}-07$ & $1.99 \mathrm{E}-07$ & $1.99 \mathrm{E}-07$ \\
\hline Y 89M & $2.96 \mathrm{E}-13$ & $2.39 \mathrm{E}-13$ & $1.94 \mathrm{E}-13$ & $3.55 \mathrm{E}-14$ & $5.10 \mathrm{E}-16$ & $1.52 \mathrm{E}-21$ & $3.15 \mathrm{E}-25$ & $7.81 \mathrm{E}-30$ & $0.00 \mathrm{E}+00$ \\
\hline
\end{tabular}


BSU-8242 3 DPA As-Run Physics Analysis

\begin{tabular}{|c|c|c|c|c|c|c|c|c|c|}
\hline \multicolumn{10}{|c|}{ ACTIVATION PRODUCTS (grams) } \\
\hline ISOTOPE & EOC & 1 Day & 2 Days & 10 Days & 30 Days & 90 Days & 130 Days & 180 Days & 360 Days \\
\hline Y 90 & $1.95 \mathrm{E}-08$ & $1.50 \mathrm{E}-08$ & $1.16 \mathrm{E}-08$ & $1.45 \mathrm{E}-09$ & $8.00 \mathrm{E}-12$ & $3.01 \mathrm{E}-16$ & $2.99 \mathrm{E}-16$ & $2.98 \mathrm{E}-16$ & $2.95 \mathrm{E}-16$ \\
\hline Y 90M & $2.96 \mathrm{E}-16$ & $1.39 \mathrm{E}-18$ & $6.47 \mathrm{E}-21$ & $1.47 \mathrm{E}-39$ & $0.00 \mathrm{E}+00$ & $0.00 \mathrm{E}+00$ & $0.00 \mathrm{E}+00$ & $0.00 \mathrm{E}+00$ & $0.00 \mathrm{E}+00$ \\
\hline Y 91 & $3.86 \mathrm{E}-11$ & $3.82 \mathrm{E}-11$ & $3.77 \mathrm{E}-11$ & $3.43 \mathrm{E}-11$ & $2.71 \mathrm{E}-11$ & $1.33 \mathrm{E}-11$ & $8.28 \mathrm{E}-12$ & $4.58 \mathrm{E}-12$ & $5.43 \mathrm{E}-13$ \\
\hline Y 92 & $5.36 \mathrm{E}-14$ & $4.87 \mathrm{E}-16$ & $4.43 \mathrm{E}-18$ & $2.06 \mathrm{E}-34$ & $0.00 \mathrm{E}+00$ & $0.00 \mathrm{E}+00$ & $0.00 \mathrm{E}+00$ & $0.00 \mathrm{E}+00$ & $0.00 \mathrm{E}+00$ \\
\hline Y 94 & $2.76 \mathrm{E}-17$ & $0.00 \mathrm{E}+00$ & $0.00 \mathrm{E}+00$ & $0.00 \mathrm{E}+00$ & $0.00 \mathrm{E}+00$ & $0.00 \mathrm{E}+00$ & $0.00 \mathrm{E}+00$ & $0.00 \mathrm{E}+00$ & $0.00 \mathrm{E}+00$ \\
\hline ZR 89 & $5.21 \mathrm{E}-09$ & $4.21 \mathrm{E}-09$ & $3.41 \mathrm{E}-09$ & $6.25 \mathrm{E}-10$ & 8.99E-12 & $2.68 \mathrm{E}-17$ & $5.54 \mathrm{E}-21$ & $1.38 \mathrm{E}-25$ & $3.62 \mathrm{E}-42$ \\
\hline ZR 90 & $8.96 \mathrm{E}-07$ & $9.00 \mathrm{E}-07$ & $9.04 \mathrm{E}-07$ & $9.14 \mathrm{E}-07$ & $9.15 \mathrm{E}-07$ & $9.15 \mathrm{E}-07$ & $9.15 \mathrm{E}-07$ & $9.15 \mathrm{E}-07$ & $9.15 \mathrm{E}-07$ \\
\hline ZR 91 & $2.71 \mathrm{E}-06$ & $2.71 \mathrm{E}-06$ & $2.71 \mathrm{E}-06$ & $2.71 \mathrm{E}-06$ & $2.71 \mathrm{E}-06$ & $2.71 \mathrm{E}-06$ & $2.71 \mathrm{E}-06$ & $2.71 \mathrm{E}-06$ & $2.71 \mathrm{E}-06$ \\
\hline ZR 92 & $6.31 \mathrm{E}-05$ & $6.33 \mathrm{E}-05$ & $6.35 \mathrm{E}-05$ & $6.48 \mathrm{E}-05$ & $6.60 \mathrm{E}-05$ & $6.64 \mathrm{E}-05$ & $6.64 \mathrm{E}-05$ & $6.64 \mathrm{E}-05$ & $6.64 \mathrm{E}-05$ \\
\hline ZR 93 & $1.12 \mathrm{E}-05$ & $1.12 \mathrm{E}-05$ & $1.12 \mathrm{E}-05$ & $1.12 \mathrm{E}-05$ & $1.12 \mathrm{E}-05$ & $1.12 \mathrm{E}-05$ & $1.12 \mathrm{E}-05$ & $1.12 \mathrm{E}-05$ & $1.12 \mathrm{E}-05$ \\
\hline ZR 94 & $5.80 \mathrm{E}-06$ & $5.80 \mathrm{E}-06$ & $5.80 \mathrm{E}-06$ & $5.80 \mathrm{E}-06$ & $5.80 \mathrm{E}-06$ & $5.80 \mathrm{E}-06$ & $5.80 \mathrm{E}-06$ & $5.80 \mathrm{E}-06$ & $5.80 \mathrm{E}-06$ \\
\hline ZR 95 & $2.52 \mathrm{E}-07$ & $2.49 \mathrm{E}-07$ & $2.46 \mathrm{E}-07$ & $2.26 \mathrm{E}-07$ & $1.82 \mathrm{E}-07$ & $9.49 \mathrm{E}-08$ & $6.15 \mathrm{E}-08$ & $3.58 \mathrm{E}-08$ & $5.09 \mathrm{E}-09$ \\
\hline ZR 96 & $4.73 \mathrm{E}-10$ & $4.73 \mathrm{E}-10$ & $4.73 \mathrm{E}-10$ & $4.73 \mathrm{E}-10$ & $4.73 \mathrm{E}-10$ & $4.73 \mathrm{E}-10$ & $4.73 \mathrm{E}-10$ & $4.73 \mathrm{E}-10$ & 4.73E-10 \\
\hline ZR 97 & $9.66 \mathrm{E}-11$ & $3.61 \mathrm{E}-11$ & $1.35 \mathrm{E}-11$ & $5.13 \mathrm{E}-15$ & $1.45 \mathrm{E}-23$ & $0.00 \mathrm{E}+00$ & $0.00 \mathrm{E}+00$ & $0.00 \mathrm{E}+00$ & $0.00 \mathrm{E}+00$ \\
\hline NB 92 & $3.28 \mathrm{E}-06$ & $3.07 \mathrm{E}-06$ & $2.87 \mathrm{E}-06$ & $1.66 \mathrm{E}-06$ & $4.24 \mathrm{E}-07$ & $7.08 \mathrm{E}-09$ & $4.62 \mathrm{E}-10$ & $1.53 \mathrm{E}-11$ & $7.08 \mathrm{E}-17$ \\
\hline NB 93 & $3.47 \mathrm{E}+00$ & $3.47 \mathrm{E}+00$ & $3.47 \mathrm{E}+00$ & $3.47 \mathrm{E}+00$ & $3.47 \mathrm{E}+00$ & $3.47 \mathrm{E}+00$ & $3.47 \mathrm{E}+00$ & $3.47 \mathrm{E}+00$ & $3.47 \mathrm{E}+00$ \\
\hline NB 93M & $2.24 \mathrm{E}-12$ & $2.26 \mathrm{E}-12$ & $2.27 \mathrm{E}-12$ & $2.37 \mathrm{E}-12$ & $2.63 \mathrm{E}-12$ & $3.39 \mathrm{E}-12$ & $3.90 \mathrm{E}-12$ & $4.52 \mathrm{E}-12$ & $6.75 \mathrm{E}-12$ \\
\hline NB 94 & $2.05 \mathrm{E}-02$ & $2.05 \mathrm{E}-02$ & $2.05 \mathrm{E}-02$ & $2.05 \mathrm{E}-02$ & $2.05 \mathrm{E}-02$ & $2.05 \mathrm{E}-02$ & $2.05 \mathrm{E}-02$ & $2.05 \mathrm{E}-02$ & $2.05 \mathrm{E}-02$ \\
\hline NB 95 & $2.42 \mathrm{E}-04$ & $2.38 \mathrm{E}-04$ & $2.33 \mathrm{E}-04$ & $1.99 \mathrm{E}-04$ & $1.34 \mathrm{E}-04$ & $4.12 \mathrm{E}-05$ & $1.87 \mathrm{E}-05$ & $7.00 \mathrm{E}-06$ & $2.06 \mathrm{E}-07$ \\
\hline NB 95M & $1.01 \mathrm{E}-10$ & $1.01 \mathrm{E}-10$ & $1.00 \mathrm{E}-10$ & $9.38 \mathrm{E}-11$ & $7.60 \mathrm{E}-11$ & $3.97 \mathrm{E}-11$ & $2.58 \mathrm{E}-11$ & $1.50 \mathrm{E}-11$ & $2.13 \mathrm{E}-12$ \\
\hline NB 96 & $3.59 \mathrm{E}-08$ & $1.76 \mathrm{E}-08$ & 8.62E-09 & $2.88 \mathrm{E}-11$ & $1.87 \mathrm{E}-17$ & $5.10 \mathrm{E}-36$ & $0.00 \mathrm{E}+00$ & $0.00 \mathrm{E}+00$ & $0.00 \mathrm{E}+00$ \\
\hline NB 97 & $2.44 \mathrm{E}-10$ & $2.58 \mathrm{E}-12$ & $9.64 \mathrm{E}-13$ & $3.93 \mathrm{E}-16$ & $1.11 \mathrm{E}-24$ & $0.00 \mathrm{E}+00$ & $0.00 \mathrm{E}+00$ & $0.00 \mathrm{E}+00$ & $0.00 \mathrm{E}+00$ \\
\hline NB 97M & $9.02 \mathrm{E}-14$ & $3.37 \mathrm{E}-14$ & $1.26 \mathrm{E}-14$ & 4.79E-18 & $1.35 \mathrm{E}-26$ & $0.00 \mathrm{E}+00$ & $0.00 \mathrm{E}+00$ & $0.00 \mathrm{E}+00$ & $0.00 \mathrm{E}+00$ \\
\hline NB 98 & $2.38 \mathrm{E}-14$ & $0.00 \mathrm{E}+00$ & $0.00 \mathrm{E}+00$ & $0.00 \mathrm{E}+00$ & $0.00 \mathrm{E}+00$ & $0.00 \mathrm{E}+00$ & $0.00 \mathrm{E}+00$ & $0.00 \mathrm{E}+00$ & $0.00 \mathrm{E}+00$ \\
\hline NB100 & $1.03 \mathrm{E}-15$ & $0.00 \mathrm{E}+00$ & $0.00 \mathrm{E}+00$ & $0.00 \mathrm{E}+00$ & $0.00 \mathrm{E}+00$ & $0.00 \mathrm{E}+00$ & $0.00 \mathrm{E}+00$ & $0.00 \mathrm{E}+00$ & $0.00 \mathrm{E}+00$ \\
\hline MO 92 & $1.47 \mathrm{E}+00$ & $1.47 \mathrm{E}+00$ & $1.47 \mathrm{E}+00$ & $1.47 \mathrm{E}+00$ & $1.47 \mathrm{E}+00$ & $1.47 \mathrm{E}+00$ & $1.47 \mathrm{E}+00$ & $1.47 \mathrm{E}+00$ & $1.47 \mathrm{E}+00$ \\
\hline
\end{tabular}


BSU-8242 3 DPA As-Run Physics Analysis

\begin{tabular}{|c|c|c|c|c|c|c|c|c|c|}
\hline \multicolumn{10}{|c|}{ ACTIVATION PRODUCTS (grams) } \\
\hline ISOTOPE & EOC & 1 Day & 2 Days & 10 Days & 30 Days & 90 Days & 130 Days & 180 Days & 360 Days \\
\hline MO 93M & $2.75 \mathrm{E}-08$ & $2.42 \mathrm{E}-09$ & $2.14 \mathrm{E}-10$ & $7.79 \mathrm{E}-19$ & $6.28 \mathrm{E}-40$ & $0.00 \mathrm{E}+00$ & $0.00 \mathrm{E}+00$ & $0.00 \mathrm{E}+00$ & $0.00 \mathrm{E}+00$ \\
\hline MO 93 & $3.70 \mathrm{E}-04$ & $3.70 \mathrm{E}-04$ & $3.70 \mathrm{E}-04$ & $3.70 \mathrm{E}-04$ & $3.70 \mathrm{E}-04$ & $3.70 \mathrm{E}-04$ & $3.70 \mathrm{E}-04$ & $3.70 \mathrm{E}-04$ & $3.70 \mathrm{E}-04$ \\
\hline MO 94 & $9.46 \mathrm{E}-01$ & $9.46 \mathrm{E}-01$ & $9.46 \mathrm{E}-01$ & $9.46 \mathrm{E}-01$ & $9.46 \mathrm{E}-01$ & $9.46 \mathrm{E}-01$ & $9.46 \mathrm{E}-01$ & $9.46 \mathrm{E}-01$ & $9.46 \mathrm{E}-01$ \\
\hline MO 95 & $1.54 \mathrm{E}+00$ & $1.54 \mathrm{E}+00$ & $1.54 \mathrm{E}+00$ & $1.54 \mathrm{E}+00$ & $1.54 \mathrm{E}+00$ & $1.54 \mathrm{E}+00$ & $1.54 \mathrm{E}+00$ & $1.54 \mathrm{E}+00$ & $1.54 \mathrm{E}+00$ \\
\hline MO 96 & $1.82 \mathrm{E}+00$ & $1.82 \mathrm{E}+00$ & $1.82 \mathrm{E}+00$ & $1.82 \mathrm{E}+00$ & $1.82 \mathrm{E}+00$ & $1.82 \mathrm{E}+00$ & $1.82 \mathrm{E}+00$ & $1.82 \mathrm{E}+00$ & $1.82 \mathrm{E}+00$ \\
\hline MO 97 & $1.02 \mathrm{E}+00$ & $1.02 \mathrm{E}+00$ & $1.02 \mathrm{E}+00$ & $1.02 \mathrm{E}+00$ & $1.02 \mathrm{E}+00$ & $1.02 \mathrm{E}+00$ & $1.02 \mathrm{E}+00$ & $1.02 \mathrm{E}+00$ & $1.02 \mathrm{E}+00$ \\
\hline MO 98 & $2.56 \mathrm{E}+00$ & $2.56 \mathrm{E}+00$ & $2.56 \mathrm{E}+00$ & $2.56 \mathrm{E}+00$ & $2.56 \mathrm{E}+00$ & $2.56 \mathrm{E}+00$ & $2.56 \mathrm{E}+00$ & $2.56 \mathrm{E}+00$ & $2.56 \mathrm{E}+00$ \\
\hline MO 99 & $1.97 \mathrm{E}-04$ & $1.53 \mathrm{E}-04$ & $1.19 \mathrm{E}-04$ & $1.59 \mathrm{E}-05$ & $1.03 \mathrm{E}-07$ & $2.77 \mathrm{E}-14$ & $1.16 \mathrm{E}-18$ & $3.90 \mathrm{E}-24$ & $1.39 \mathrm{E}-43$ \\
\hline MO100 & $1.04 \mathrm{E}+00$ & $1.04 \mathrm{E}+00$ & $1.04 \mathrm{E}+00$ & $1.04 \mathrm{E}+00$ & $1.04 \mathrm{E}+00$ & $1.04 \mathrm{E}+00$ & $1.04 \mathrm{E}+00$ & $1.04 \mathrm{E}+00$ & $1.04 \mathrm{E}+00$ \\
\hline MO101 & $1.85 \mathrm{E}-07$ & $0.00 \mathrm{E}+00$ & $0.00 \mathrm{E}+00$ & $0.00 \mathrm{E}+00$ & $0.00 \mathrm{E}+00$ & $0.00 \mathrm{E}+00$ & $0.00 \mathrm{E}+00$ & $0.00 \mathrm{E}+00$ & $0.00 \mathrm{E}+00$ \\
\hline TC 99 & $1.02 \mathrm{E}-03$ & $1.03 \mathrm{E}-03$ & $1.03 \mathrm{E}-03$ & $1.04 \mathrm{E}-03$ & $1.05 \mathrm{E}-03$ & $1.05 \mathrm{E}-03$ & $1.05 \mathrm{E}-03$ & $1.05 \mathrm{E}-03$ & $1.05 \mathrm{E}-03$ \\
\hline TC100 & $2.02 \mathrm{E}-10$ & $0.00 \mathrm{E}+00$ & $0.00 \mathrm{E}+00$ & $0.00 \mathrm{E}+00$ & $0.00 \mathrm{E}+00$ & $0.00 \mathrm{E}+00$ & $0.00 \mathrm{E}+00$ & $0.00 \mathrm{E}+00$ & $0.00 \mathrm{E}+00$ \\
\hline TC101 & $1.79 \mathrm{E}-07$ & $0.00 \mathrm{E}+00$ & $0.00 \mathrm{E}+00$ & $0.00 \mathrm{E}+00$ & $0.00 \mathrm{E}+00$ & $0.00 \mathrm{E}+00$ & $0.00 \mathrm{E}+00$ & $0.00 \mathrm{E}+00$ & $0.00 \mathrm{E}+00$ \\
\hline RU 99 & $1.60 \mathrm{E}-09$ & $1.61 \mathrm{E}-09$ & $1.62 \mathrm{E}-09$ & $1.69 \mathrm{E}-09$ & $1.88 \mathrm{E}-09$ & $2.44 \mathrm{E}-09$ & $2.81 \mathrm{E}-09$ & $3.28 \mathrm{E}-09$ & 4.95E-09 \\
\hline RU100 & 7.02E-05 & $7.02 \mathrm{E}-05$ & $7.02 \mathrm{E}-05$ & $7.02 \mathrm{E}-05$ & $7.02 \mathrm{E}-05$ & $7.02 \mathrm{E}-05$ & 7.02E-05 & 7.02E-05 & $7.02 \mathrm{E}-05$ \\
\hline RU101 & $2.23 \mathrm{E}-03$ & $2.23 \mathrm{E}-03$ & $2.23 \mathrm{E}-03$ & $2.23 \mathrm{E}-03$ & $2.23 \mathrm{E}-03$ & $2.23 \mathrm{E}-03$ & $2.23 \mathrm{E}-03$ & $2.23 \mathrm{E}-03$ & $2.23 \mathrm{E}-03$ \\
\hline RU102 & $4.88 \mathrm{E}-05$ & $4.88 \mathrm{E}-05$ & $4.88 \mathrm{E}-05$ & $4.88 \mathrm{E}-05$ & $4.88 \mathrm{E}-05$ & $4.88 \mathrm{E}-05$ & $4.88 \mathrm{E}-05$ & $4.88 \mathrm{E}-05$ & $4.88 \mathrm{E}-05$ \\
\hline RU103 & 3.13E-08 & $3.08 \mathrm{E}-08$ & $3.02 \mathrm{E}-08$ & $2.62 \mathrm{E}-08$ & $1.84 \mathrm{E}-08$ & $6.40 \mathrm{E}-09$ & $3.16 \mathrm{E}-09$ & $1.31 \mathrm{E}-09$ & $5.46 \mathrm{E}-11$ \\
\hline RU104 & $3.11 \mathrm{E}-10$ & $3.11 \mathrm{E}-10$ & $3.11 \mathrm{E}-10$ & $3.11 \mathrm{E}-10$ & $3.11 \mathrm{E}-10$ & $3.11 \mathrm{E}-10$ & $3.11 \mathrm{E}-10$ & $3.11 \mathrm{E}-10$ & $3.11 \mathrm{E}-10$ \\
\hline RU105 & $1.78 \mathrm{E}-15$ & $4.20 \mathrm{E}-17$ & $9.89 \mathrm{E}-19$ & $9.43 \mathrm{E}-32$ & $0.00 \mathrm{E}+00$ & $0.00 \mathrm{E}+00$ & $0.00 \mathrm{E}+00$ & $0.00 \mathrm{E}+00$ & $0.00 \mathrm{E}+00$ \\
\hline RH103 & 2.73E-09 & $2.78 \mathrm{E}-09$ & $2.84 \mathrm{E}-09$ & $3.23 \mathrm{E}-09$ & 4.01E-09 & $5.21 \mathrm{E}-09$ & $5.53 \mathrm{E}-09$ & $5.71 \mathrm{E}-09$ & $5.84 \mathrm{E}-09$ \\
\hline RH104 & $6.26 \mathrm{E}-15$ & $0.00 \mathrm{E}+00$ & $0.00 \mathrm{E}+00$ & $0.00 \mathrm{E}+00$ & $0.00 \mathrm{E}+00$ & $0.00 \mathrm{E}+00$ & $0.00 \mathrm{E}+00$ & $0.00 \mathrm{E}+00$ & $0.00 \mathrm{E}+00$ \\
\hline RH104M & $2.79 \mathrm{E}-15$ & $0.00 \mathrm{E}+00$ & $0.00 \mathrm{E}+00$ & $0.00 \mathrm{E}+00$ & $0.00 \mathrm{E}+00$ & $0.00 \mathrm{E}+00$ & $0.00 \mathrm{E}+00$ & $0.00 \mathrm{E}+00$ & $0.00 \mathrm{E}+00$ \\
\hline RH105 & $1.13 \mathrm{E}-14$ & $8.27 \mathrm{E}-15$ & $5.20 \mathrm{E}-15$ & $1.21 \mathrm{E}-16$ & $9.89 \mathrm{E}-21$ & $5.45 \mathrm{E}-33$ & $3.66 \mathrm{E}-41$ & $0.00 \mathrm{E}+00$ & $0.00 \mathrm{E}+00$ \\
\hline RH106M & $6.04 \mathrm{E}-17$ & $3.14 \mathrm{E}-20$ & $1.63 \mathrm{E}-23$ & $0.00 \mathrm{E}+00$ & $0.00 \mathrm{E}+00$ & $0.00 \mathrm{E}+00$ & $0.00 \mathrm{E}+00$ & $0.00 \mathrm{E}+00$ & $0.00 \mathrm{E}+00$ \\
\hline PD104 & $4.11 \mathrm{E}-10$ & $4.11 \mathrm{E}-10$ & $4.11 \mathrm{E}-10$ & $4.11 \mathrm{E}-10$ & $4.11 \mathrm{E}-10$ & $4.11 \mathrm{E}-10$ & $4.11 \mathrm{E}-10$ & $4.11 \mathrm{E}-10$ & 4.11E-10 \\
\hline
\end{tabular}


BSU-8242 3 DPA As-Run Physics Analysis

\begin{tabular}{|c|c|c|c|c|c|c|c|c|c|}
\hline \multicolumn{10}{|c|}{ ACTIVATION PRODUCTS (grams) } \\
\hline ISOTOPE & EOC & 1 Day & 2 Days & 10 Days & 30 Days & 90 Days & 130 Days & 180 Days & 360 Days \\
\hline PD105 & $9.65 \mathrm{E}-13$ & $9.69 \mathrm{E}-13$ & $9.73 \mathrm{E}-13$ & $9.78 \mathrm{E}-13$ & $9.78 \mathrm{E}-13$ & $9.78 \mathrm{E}-13$ & $9.78 \mathrm{E}-13$ & $9.78 \mathrm{E}-13$ & $9.78 \mathrm{E}-13$ \\
\hline PD106 & $5.90 \mathrm{E}-14$ & $5.91 \mathrm{E}-14$ & $5.91 \mathrm{E}-14$ & $5.91 \mathrm{E}-14$ & $5.91 \mathrm{E}-14$ & $5.91 \mathrm{E}-14$ & $5.91 \mathrm{E}-14$ & $5.91 \mathrm{E}-14$ & $5.91 \mathrm{E}-14$ \\
\hline PD107 & $4.10 \mathrm{E}-17$ & $4.10 \mathrm{E}-17$ & $4.10 \mathrm{E}-17$ & $4.10 \mathrm{E}-17$ & $4.10 \mathrm{E}-17$ & $4.10 \mathrm{E}-17$ & $4.10 \mathrm{E}-17$ & $4.10 \mathrm{E}-17$ & $4.10 \mathrm{E}-17$ \\
\hline TA180 & $1.83 \mathrm{E}-04$ & $1.83 \mathrm{E}-04$ & $1.83 \mathrm{E}-04$ & $1.83 \mathrm{E}-04$ & $1.83 \mathrm{E}-04$ & $1.83 \mathrm{E}-04$ & $1.83 \mathrm{E}-04$ & $1.83 \mathrm{E}-04$ & $1.83 \mathrm{E}-04$ \\
\hline TA181 & $3.28 \mathrm{E}+00$ & $3.28 \mathrm{E}+00$ & $3.28 \mathrm{E}+00$ & $3.28 \mathrm{E}+00$ & $3.28 \mathrm{E}+00$ & $3.28 \mathrm{E}+00$ & $3.28 \mathrm{E}+00$ & $3.28 \mathrm{E}+00$ & $3.28 \mathrm{E}+00$ \\
\hline TA182 & $8.47 \mathrm{E}-02$ & $8.42 \mathrm{E}-02$ & 8.37E-02 & 7.97E-02 & 7.07E-02 & 4.92E-02 & $3.87 \mathrm{E}-02$ & $2.86 \mathrm{E}-02$ & $9.67 \mathrm{E}-03$ \\
\hline TA182M & $4.16 \mathrm{E}-08$ & $0.00 \mathrm{E}+00$ & $0.00 \mathrm{E}+00$ & $0.00 \mathrm{E}+00$ & $0.00 \mathrm{E}+00$ & $0.00 \mathrm{E}+00$ & $0.00 \mathrm{E}+00$ & $0.00 \mathrm{E}+00$ & $0.00 \mathrm{E}+00$ \\
\hline TA183 & $3.56 \mathrm{E}-02$ & $3.10 \mathrm{E}-02$ & $2.71 \mathrm{E}-02$ & $9.13 \mathrm{E}-03$ & $6.03 \mathrm{E}-04$ & $1.73 \mathrm{E}-07$ & $7.55 \mathrm{E}-10$ & $8.41 \mathrm{E}-13$ & $2.00 \mathrm{E}-23$ \\
\hline W180 & $1.59 \mathrm{E}-17$ & $1.59 \mathrm{E}-17$ & $1.59 \mathrm{E}-17$ & $1.61 \mathrm{E}-17$ & $1.65 \mathrm{E}-17$ & $1.78 \mathrm{E}-17$ & $1.87 \mathrm{E}-17$ & $1.98 \mathrm{E}-17$ & $2.37 \mathrm{E}-17$ \\
\hline W182 & $1.54 \mathrm{E}-01$ & $1.54 \mathrm{E}-01$ & $1.55 \mathrm{E}-01$ & $1.59 \mathrm{E}-01$ & $1.68 \mathrm{E}-01$ & $1.89 \mathrm{E}-01$ & $2.00 \mathrm{E}-01$ & $2.10 \mathrm{E}-01$ & $2.29 \mathrm{E}-01$ \\
\hline W183M & $4.98 \mathrm{E}-11$ & $0.00 \mathrm{E}+00$ & $0.00 \mathrm{E}+00$ & $0.00 \mathrm{E}+00$ & $0.00 \mathrm{E}+00$ & $0.00 \mathrm{E}+00$ & $0.00 \mathrm{E}+00$ & $0.00 \mathrm{E}+00$ & $0.00 \mathrm{E}+00$ \\
\hline W183 & $8.37 \mathrm{E}-01$ & $8.42 \mathrm{E}-01$ & $8.46 \mathrm{E}-01$ & 8.64E-01 & $8.72 \mathrm{E}-01$ & $8.73 \mathrm{E}-01$ & $8.73 \mathrm{E}-01$ & $8.73 \mathrm{E}-01$ & $8.73 \mathrm{E}-01$ \\
\hline W184 & $5.53 \mathrm{E}-02$ & $5.53 \mathrm{E}-02$ & $5.53 \mathrm{E}-02$ & $5.53 \mathrm{E}-02$ & $5.53 \mathrm{E}-02$ & $5.53 \mathrm{E}-02$ & $5.53 \mathrm{E}-02$ & $5.53 \mathrm{E}-02$ & $5.53 \mathrm{E}-02$ \\
\hline W185 & $8.42 \mathrm{E}-05$ & $8.34 \mathrm{E}-05$ & $8.26 \mathrm{E}-05$ & 7.67E-05 & $6.38 \mathrm{E}-05$ & $3.67 \mathrm{E}-05$ & $2.54 \mathrm{E}-05$ & $1.60 \mathrm{E}-05$ & $3.03 \mathrm{E}-06$ \\
\hline $\mathrm{W} 185 \mathrm{M}$ & $4.07 \mathrm{E}-12$ & $0.00 \mathrm{E}+00$ & $0.00 \mathrm{E}+00$ & $0.00 \mathrm{E}+00$ & $0.00 \mathrm{E}+00$ & $0.00 \mathrm{E}+00$ & $0.00 \mathrm{E}+00$ & $0.00 \mathrm{E}+00$ & $0.00 \mathrm{E}+00$ \\
\hline W186 & $3.91 \mathrm{E}-07$ & $4.00 \mathrm{E}-07$ & $4.08 \mathrm{E}-07$ & $4.36 \mathrm{E}-07$ & 4.44E-07 & 4.44E-07 & 4.44E-07 & 4.44E-07 & 4.44E-07 \\
\hline W187 & $6.99 \mathrm{E}-10$ & $3.48 \mathrm{E}-10$ & $1.74 \mathrm{E}-10$ & $6.63 \mathrm{E}-13$ & $5.96 \mathrm{E}-19$ & $4.35 \mathrm{E}-37$ & $0.00 \mathrm{E}+00$ & $0.00 \mathrm{E}+00$ & $0.00 \mathrm{E}+00$ \\
\hline W188 & $1.10 \mathrm{E}-10$ & $1.09 \mathrm{E}-10$ & $1.08 \mathrm{E}-10$ & $9.95 \mathrm{E}-11$ & $8.15 \mathrm{E}-11$ & $4.47 \mathrm{E}-11$ & $3.00 \mathrm{E}-11$ & $1.82 \mathrm{E}-11$ & $3.02 \mathrm{E}-12$ \\
\hline RE185 & $3.53 \mathrm{E}-05$ & $3.61 \mathrm{E}-05$ & $3.68 \mathrm{E}-05$ & $4.27 \mathrm{E}-05$ & $5.57 \mathrm{E}-05$ & $8.28 \mathrm{E}-05$ & $9.41 \mathrm{E}-05$ & $1.04 \mathrm{E}-04$ & $1.16 \mathrm{E}-04$ \\
\hline RE186 & $8.16 \mathrm{E}-07$ & $6.79 \mathrm{E}-07$ & $5.65 \mathrm{E}-07$ & $1.30 \mathrm{E}-07$ & $3.31 \mathrm{E}-09$ & $5.47 \mathrm{E}-14$ & $3.54 \mathrm{E}-17$ & $3.67 \mathrm{E}-21$ & $1.65 \mathrm{E}-35$ \\
\hline RE187 & $1.66 \mathrm{E}-08$ & $1.69 \mathrm{E}-08$ & $1.71 \mathrm{E}-08$ & $1.73 \mathrm{E}-08$ & $1.73 \mathrm{E}-08$ & $1.73 \mathrm{E}-08$ & $1.73 \mathrm{E}-08$ & $1.73 \mathrm{E}-08$ & $1.73 \mathrm{E}-08$ \\
\hline RE188 & $1.96 \mathrm{E}-11$ & $8.16 \mathrm{E}-12$ & $3.75 \mathrm{E}-12$ & $1.03 \mathrm{E}-12$ & $8.39 \mathrm{E}-13$ & $4.61 \mathrm{E}-13$ & $3.09 \mathrm{E}-13$ & $1.88 \mathrm{E}-13$ & $3.11 \mathrm{E}-14$ \\
\hline RE188M & $3.31 \mathrm{E}-13$ & $0.00 \mathrm{E}+00$ & $0.00 \mathrm{E}+00$ & $0.00 \mathrm{E}+00$ & $0.00 \mathrm{E}+00$ & $0.00 \mathrm{E}+00$ & $0.00 \mathrm{E}+00$ & $0.00 \mathrm{E}+00$ & $0.00 \mathrm{E}+00$ \\
\hline RE189 & $4.28 \mathrm{E}-16$ & $2.16 \mathrm{E}-16$ & $1.09 \mathrm{E}-16$ & $4.56 \mathrm{E}-19$ & $5.16 \mathrm{E}-25$ & $7.95 \mathrm{E}-43$ & $0.00 \mathrm{E}+00$ & $0.00 \mathrm{E}+00$ & $0.00 \mathrm{E}+00$ \\
\hline OS186 & $5.88 \mathrm{E}-06$ & $6.01 \mathrm{E}-06$ & $6.12 \mathrm{E}-06$ & $6.52 \mathrm{E}-06$ & $6.64 \mathrm{E}-06$ & 6.64E-06 & $6.64 \mathrm{E}-06$ & $6.64 \mathrm{E}-06$ & $6.64 \mathrm{E}-06$ \\
\hline OS188 & $5.50 \mathrm{E}-10$ & $5.63 \mathrm{E}-10$ & $5.69 \mathrm{E}-10$ & $5.80 \mathrm{E}-10$ & $5.98 \mathrm{E}-10$ & $6.35 \mathrm{E}-10$ & $6.50 \mathrm{E}-10$ & $6.62 \mathrm{E}-10$ & $6.77 \mathrm{E}-10$ \\
\hline
\end{tabular}


BSU-8242 3 DPA As-Run Physics Analysis

\begin{tabular}{|c|c|c|c|c|c|c|c|c|c|}
\hline \multicolumn{10}{|c|}{ ACTIVATION PRODUCTS (grams) } \\
\hline ISOTOPE & EOC & 1 Day & 2 Days & 10 Days & 30 Days & 90 Days & 130 Days & 180 Days & 360 Days \\
\hline OS189 & $3.98 \mathrm{E}-12$ & $3.98 \mathrm{E}-12$ & $3.98 \mathrm{E}-12$ & $3.98 \mathrm{E}-12$ & $3.98 \mathrm{E}-12$ & $3.98 \mathrm{E}-12$ & $3.98 \mathrm{E}-12$ & $3.98 \mathrm{E}-12$ & $3.98 \mathrm{E}-12$ \\
\hline OS190 & $1.43 \mathrm{E}-13$ & $1.43 \mathrm{E}-13$ & $1.43 \mathrm{E}-13$ & $1.43 \mathrm{E}-13$ & $1.43 \mathrm{E}-13$ & $1.43 \mathrm{E}-13$ & $1.43 \mathrm{E}-13$ & $1.43 \mathrm{E}-13$ & $1.43 \mathrm{E}-13$ \\
\hline OS191 & $2.16 \mathrm{E}-16$ & $2.14 \mathrm{E}-16$ & $2.07 \mathrm{E}-16$ & $1.45 \mathrm{E}-16$ & $5.90 \mathrm{E}-17$ & $3.97 \mathrm{E}-18$ & $6.56 \mathrm{E}-19$ & $6.92 \mathrm{E}-20$ & $2.10 \mathrm{E}-23$ \\
\hline OS191M & $1.13 \mathrm{E}-17$ & $3.13 \mathrm{E}-18$ & $8.71 \mathrm{E}-19$ & $3.12 \mathrm{E}-23$ & $2.39 \mathrm{E}-34$ & $0.00 \mathrm{E}+00$ & $0.00 \mathrm{E}+00$ & $0.00 \mathrm{E}+00$ & $0.00 \mathrm{E}+00$ \\
\hline IR191 & $1.42 \mathrm{E}-16$ & $1.52 \mathrm{E}-16$ & $1.61 \mathrm{E}-16$ & $2.24 \mathrm{E}-16$ & $3.10 \mathrm{E}-16$ & $3.65 \mathrm{E}-16$ & $3.69 \mathrm{E}-16$ & $3.69 \mathrm{E}-16$ & $3.69 \mathrm{E}-16$ \\
\hline IR192 & $2.51 \mathrm{E}-17$ & $2.49 \mathrm{E}-17$ & $2.46 \mathrm{E}-17$ & $2.29 \mathrm{E}-17$ & $1.90 \mathrm{E}-17$ & $1.08 \mathrm{E}-17$ & 7.43E-18 & $4.65 \mathrm{E}-18$ & $8.62 \mathrm{E}-19$ \\
\hline PT192 & $3.50 \mathrm{E}-18$ & $3.73 \mathrm{E}-18$ & $3.95 \mathrm{E}-18$ & $5.64 \mathrm{E}-18$ & $9.36 \mathrm{E}-18$ & $1.71 \mathrm{E}-17$ & $2.03 \mathrm{E}-17$ & $2.30 \mathrm{E}-17$ & $2.66 \mathrm{E}-17$ \\
\hline SUMTOT & $8.67 \mathrm{E}+02$ & $8.67 \mathrm{E}+02$ & $8.67 \mathrm{E}+02$ & $8.67 \mathrm{E}+02$ & $8.67 \mathrm{E}+02$ & $8.67 \mathrm{E}+02$ & $8.67 \mathrm{E}+02$ & $8.67 \mathrm{E}+02$ & $8.67 \mathrm{E}+02$ \\
\hline 0TOTAL & $8.67 \mathrm{E}+02$ & $8.67 \mathrm{E}+02$ & $8.67 \mathrm{E}+02$ & $8.67 \mathrm{E}+02$ & $8.67 \mathrm{E}+02$ & $8.67 \mathrm{E}+02$ & $8.67 \mathrm{E}+02$ & $8.67 \mathrm{E}+02$ & $8.67 \mathrm{E}+02$ \\
\hline
\end{tabular}




\section{REFERENCES}

[1] R.R. Little, Interoffice Memorandum, “Advanced Test Reactor Power History Through Cycle 166B-1," Rev. 0, January 15, 2020.

[2] F. Brown, B. Kiedrowsky, J. Bull, et al, "MCNP5-1.60 Release Notes,” LA-UR-10-06235 (2010).

[3] X-5 Monte Carlo Team, "MCNP - A General Monte Carlo N-Particle Transport Code, Version 5,” Volume I, LA-UR-03-1987, Los Alamos National Laboratory, April 24, 2003 (Revised 10/3/05) and Volume II, LA-CP-0245, Los Alamos National Laboratory, April 24, 2003 (Revised 10/3/05) (Vol. II available with a licensed copy of MCNP).

[4] E.T. Boulette and W.L. Bunch, Analysis of ZPPR/FTR Shield Experiments Gamma Distributions, WHAN-FR-13, February 1971.

[5] GDE-594, "Experiment Design and Analysis Guide - Neutronics \& Physics," Rev. 1, June 26, 2015.

[6] E.A. Read, C.R.E. de Oliveira, "A Functional Method for Estimating DPA Tallies in Monte Carlo Calculations of Light Water Reactors," International Conference on Mathematics and Computational Methods Applied to Nuclear Science and Engineering (M\&C 2011), Rio de Janeiro, Brazil, 2011.

[7] R.E. MacFarlane, D.W. Muir, "The NJOY Nuclear Data Processing System, Version 99," LA-1270, Los Alamos National Laboratory (2002).

[8] P.F. Rose, "ENDF-201, ENDF/B-VI Summary Documentation, Version 4,” BNL-NCS 17541, Brookhaven National Laboratory (1996).

[9] M.B. Chadwick et al., "ENDF/B-VII.0: Next Generation Evaluated Nuclear Data Library for Nuclear Science and Technology," Nuclear Data Sheets, Vol. 107, Issue 12, December 2006.

[10] R.E. Macfarlane, D.W. Muir, F.M. Mann, "Radiation Damage Calculations with NJOY," Journal of Nuclear Materials, 122 (1984), 1041-1046.

[11] FOR-306, “Boise State University-8242 Irradiation Experiment”, Functional \& Operational Requirements, Rev. 0, December 2016.

[12] Jason Brookman, "As-Run Physics Analysis for the Utah State University Project in the Advanced Test Reactor,” ECAR-3050, Rev. 01, March 2016.

[13] T. Nakagawa, et al., "Japanese Evaluated Nuclear* Data Library Version 3 Revision-2: JENDL3.2," Journal of Nuclear Science and Technology, 32, pp. 1259-1271 (December 1995).

[14] J.W. Nielsen, "Verification and Validation Testing of MCNP and ORIGEN2 Computer Codes for Idaho National Laboratory (INO) High Performance Computing (HPC) Systems," TEV2944, Revision 04, July 2019. 


\section{ATTACHMENT A}

\section{$\overline{\overline{\text { INTEROFFICE MEMORANDUM }} \quad \text { Idaho National laboratory }}$}

Date: January 15,2020

To: $\quad$ R. R. Little, Reactor and Nuclear Safety Engineering Manager, Advanced Test Reactor Programs

From: $\quad$ Reactor Engineering, Advanced Test Reactor Programs

Subject: $\quad$ Advanced Test Reactor Power History Through Cycle 166B-1

References: (a) A. V. Briscoe letter to J. L. Durney, ATR Power History Through Cycle 34C-1, June 7, 1977, AVB-9-77

(b) C. C. Swanson letter to J. L. Durney, ATR Power History Through Cycle 72A-1, February 3, 1986, CAS-05-86

(c) L. S. Loret letter to E. C. Anderson, Sr., ATR Power History Through Cycle 102B-1, February 28, 1994, LSL-11-94

(d) D. E. Hale letter to J. C. Chapman, ATR Power History Through Cycle 133B-1, August 18, 2004, DEH-05-04

Table 1 lists the Advanced Test Reactor (ATR) N-16 constrained power history data since the Beryllium VI Core Internals Changeout (CIC) Cycle 134A-1 through Cycle 166B-1.

Table 2 lists the accumulated $\mathrm{N}-16$ lobe and total core exposure, as obtained from the ATR Data Acquisition System (RDAS) for Cycles 134A-1 through 166B-1.

Table 3 lists the startup and shutdown dates and times, as obtained from logbooks or Reactor Data Accuracy System (RDAS), depending on availability.

The ATR power history prior to Cycle $134 \mathrm{~A}-1$ is presented in references (a) through (d).

Table 1. Summary of ATR Power History by Cycle

\begin{tabular}{cccccc|cccccc|c}
\multicolumn{8}{c|}{ Average Lobe Power (MW) } & \multicolumn{7}{c}{ Cycle Exposure (MWd) } & Length \\
& NW & NE & C & SW & SE & NW & NE & C & SW & SE & Total & (EFPD) \\
\hline $134 A-1$ & - & - & - & - & - & - & - & - & - & - & - & - \\
$134 A-2$ & - & - & - & - & - & 0.21 & 0.30 & 0.39 & 0.36 & 0.36 & 1.62 & - \\
$134 B-1$ & 18.0 & 18.0 & 24.5 & 23.0 & 25.0 & 452.82 & 452.24 & 615.47 & 578.48 & 628.58 & $2,727.59$ & 25.10 \\
$134 B-2$ & 18.0 & 18.0 & 25.8 & 23.0 & 25.0 & 385.62 & 385.80 & 553.50 & 493.24 & 536.07 & $2,354.23$ & 21.40 \\
$135 A-1$ & 18.0 & 18.0 & 29.8 & 28.1 & 35.4 & 236.16 & 235.99 & 391.07 & 368.63 & 463.98 & $1,695.83$ & 13.10 \\
$135 B-1$ & 18.0 & 18.0 & 24.8 & 23.0 & 25.0 & 458.28 & 458.35 & 630.79 & 585.27 & 636.51 & $2,769.20$ & 25.50 \\
$135 B-2$ & 18.0 & 18.0 & 25.2 & 23.0 & 25.0 & 405.02 & 405.70 & 567.38 & 517.82 & 562.99 & $2,458.91$ & 22.50 \\
$135 \mathrm{C}-1$ & 18.0 & 18.0 & 25.0 & 23.0 & 25.0 & 729.91 & 729.70 & $1,013.45$ & 933.00 & $1,013.86$ & $4,419.92$ & 40.60 \\
$136 A-1$ & 18.0 & 18.0 & 24.0 & 23.0 & 23.0 & 916.18 & 916.39 & $1,218.91$ & $1,169.47$ & $1,170.17$ & $5,391.12$ & 50.90 \\
$136 B-1$ & 18.0 & 18.0 & 23.9 & 23.0 & 23.0 & 701.94 & 702.32 & 931.19 & 896.93 & 896.97 & $4,129.35$ & 39.00 \\
$137 A-1$ & 18.0 & 18.0 & 24.7 & 20.0 & 25.0 & 975.35 & 974.76 & $1,336.16$ & $1,083.20$ & $1,353.31$ & $5,722.78$ & 54.10 \\
$137 B-1$ & 20.0 & 17.9 & 35.5 & 56.6 & 30.4 & 241.97 & 217.01 & 429.62 & 685.38 & 367.63 & $1,941.61$ & 12.10 \\
$138 A-1$ & 18.0 & 18.0 & 23.6 & 23.0 & 25.0 & $1,046.87$ & $1,047.69$ & $1,370.69$ & $1,336.39$ & $1,453.47$ & $6,255.11$ & 58.10
\end{tabular}


R. R. Little January 15,2020

RE-01-20

Page 2 of 8

Table 1. Summary of ATR Power History by Cycle

\begin{tabular}{|c|c|c|c|c|c|c|c|c|c|c|c|c|}
\hline & \multicolumn{5}{|c|}{ Average Lobe Power (MW) } & \multicolumn{6}{|c|}{ Cycle Exposure (MWd) } & \multirow{2}{*}{$\begin{array}{l}\text { Length } \\
\text { (EFPD) }\end{array}$} \\
\hline & NW & $\mathrm{NE}$ & C & SW & SE & NW & $\mathrm{NE}$ & $C$ & SW & SE & Total & \\
\hline 138B-1 & 18.0 & 18.0 & 23.3 & 23.0 & 25.0 & 838.54 & 839.62 & $1,084.79$ & $1,070.94$ & $1,164.64$ & $4,998.53$ & 46.60 \\
\hline 139A-1 & 18.0 & 18.0 & 23.9 & 23.0 & 25.0 & 928.73 & 929.09 & $1,231.07$ & $1,186.27$ & $1,289.47$ & $5,564.63$ & 51.60 \\
\hline 139B-1 & 18.0 & 18.0 & 23.2 & 23.0 & 23.0 & 919.70 & 919.66 & $1,187.49$ & $1,174.88$ & $1,175.03$ & $5,376.76$ & 51.10 \\
\hline $140 \mathrm{~A}-1$ & 18.0 & 18.0 & 21.8 & 23.0 & 23.0 & 836.99 & 837.18 & $1,012.89$ & $1,069.69$ & $1,069.39$ & $4,826.14$ & 46.50 \\
\hline 140B-1 & 18.0 & 17.7 & 21.8 & 23.6 & 23.0 & 641.72 & 629.49 & 777.20 & 842.86 & 820.00 & $3,711.27$ & 35.70 \\
\hline $141 \mathrm{~A}-1$ & 18.0 & 18.0 & 23.4 & 23.0 & 23.0 & 583.25 & 583.08 & 756.83 & 745.05 & 745.45 & $3,413.66$ & 32.40 \\
\hline $142 \mathrm{~A}-1$ & 23.0 & 18.0 & 24.7 & 24.8 & 23.0 & $1,104.91$ & 864.75 & $1,186.04$ & $1,192.49$ & $1,104.04$ & $5,452.23$ & 48.00 \\
\hline 142B-1 & 23.0 & 18.0 & 25.4 & 25.4 & 25.0 & $1,196.93$ & 936.68 & $1,323.41$ & $1,322.45$ & $1,298.70$ & $6,078.17$ & 52.00 \\
\hline $143 A-1 / 2$ & 18.0 & 18.0 & 24.3 & 26.9 & 25.0 & 879.98 & 882.52 & $1,187.67$ & $1,315.44$ & $1,223.08$ & $5,488.69$ & 48.90 \\
\hline 143B-1 & 18.0 & 18.0 & 24.9 & 27.0 & 25.1 & $1,032.06$ & $1,032.58$ & $1,423.53$ & $1,543.74$ & $1,435.01$ & $6,466.92$ & 57.30 \\
\hline $144 \mathrm{~A}-1$ & 18.0 & 18.0 & 23.1 & 23.0 & 25.1 & 786.97 & 787.02 & $1,006.67$ & $1,004.53$ & $1,093.44$ & $4,678.63$ & 43.70 \\
\hline 144B-1 & 18.0 & 18.1 & 22.4 & 23.0 & 23.0 & 932.25 & 933.43 & $1,155.74$ & $1,190.72$ & $1,190.89$ & $5,403.03$ & 51.70 \\
\hline $145 \mathrm{~A}-1$ & 18.0 & 17.9 & 23.2 & 23.8 & 25.7 & 982.97 & 980.89 & $1,267.34$ & $1,299.49$ & $1,407.75$ & $5,938.44$ & 54.70 \\
\hline 145B-1 & 17.8 & 17.8 & 23.0 & 24.6 & 25.8 & $1,020.46$ & $1,019.96$ & $1,321.43$ & $1,407.79$ & $1,478.25$ & $6,247.89$ & 57.30 \\
\hline $146 \mathrm{~A}-1$ & 18.0 & 18.0 & 24.3 & 25.8 & 26.0 & 906.76 & 906.80 & $1,225.74$ & $1,300.02$ & $1,312.55$ & $5,651.87$ & 50.50 \\
\hline 146B-1 & 23.0 & 18.0 & 26.0 & 23.0 & 26.0 & 903.68 & 707.11 & $1,021.59$ & 903.85 & $1,020.96$ & $4,557.19$ & 39.20 \\
\hline $147 A-1$ & 23.0 & 18.0 & 24.1 & 20.9 & 23.0 & $1,156.86$ & 904.42 & $1,208.41$ & $1,049.42$ & $1,155.15$ & $5,474.26$ & 50.20 \\
\hline $148 \mathrm{~A}-1$ & 18.0 & 18.0 & 23.6 & 22.0 & 23.0 & 855.97 & 855.75 & $1,121.43$ & $1,043.79$ & $1,093.64$ & $4,970.58$ & 47.50 \\
\hline $148 \mathrm{~B}-1$ & 18.0 & 18.0 & 23.0 & 23.8 & 23.0 & 927.50 & 926.72 & $1,181.60$ & $1,223.98$ & $1,185.03$ & $5,444.83$ & 51.50 \\
\hline $149 A-1$ & 18.0 & 18.0 & 24.2 & 24.0 & 23.0 & 662.45 & 662.65 & 891.28 & 883.28 & 846.80 & 6.46 & 36.80 \\
\hline 149B-1 & 18.0 & 18.0 & 24.2 & 23.0 & 23.0 & 964.38 & 964.45 & $1,297.77$ & $1,231.63$ & $1,230.78$ & $5,689.01$ & 53.60 \\
\hline $150 \mathrm{~A}-1$ & 18.9 & 18.0 & 30.5 & 37.5 & 35.1 & 233.41 & 221.60 & 375.93 & 462.71 & 432.58 & 6.23 & 12.32 \\
\hline 150B-1 & 19.9 & 18.0 & 24.2 & 23.0 & 23.1 & 832.84 & 754.72 & $1,014.58$ & 964.86 & 966.00 & 3.00 & 41.87 \\
\hline $151 \mathrm{~A}-1$ & 18.9 & 14.2 & 22.0 & 23.6 & 23.0 & $1,058.63$ & 799.95 & 1,23 & $1,324.39$ & $1,2 \varepsilon$ & .02 & 56.14 \\
\hline $151 \mathrm{~B}-1 / 2$ & 18.9 & 14.5 & 22.1 & 23.0 & 23.0 & 97 & 741.75 & 1,13 & $1,181.94$ & $1,179.97$ & 9.94 & 51.30 \\
\hline $152 A-1 / 6$ & - & 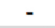 & - & - & - & 30 & 0 & 0.50 & 0 & 0.50 & 0 & 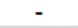 \\
\hline 152B-1 & 18.9 & 15.9 & 22.4 & 23.0 & 23.0 & 966.42 & 813.00 & $1,141.28$ & $1,172.08$ & $1,173.57$ & 6.35 & 51.02 \\
\hline 153B-1 & 19.7 & 19.7 & 30.8 & 35.4 & 44.0 & 265 & 35 & 41 & 476.12 & 59 & .56 & 13.45 \\
\hline $154 A-1$ & 20.0 & 16.0 & 20.5 & 20.5 & 23.0 & $1,048.12$ & 838.60 & $1,0 G$ & $1,071.42$ & .22 & 1.74 & 52.28 \\
\hline 154B-1 & 20.0 & 17.9 & 21.6 & 22.8 & 23.9 & $1,066.60$ & 958.47 & 1,15 & $1,220.49$ & 1,27 & 7.23 & 53.44 \\
\hline $155 \mathrm{~A}-1$ & 17.5 & 18.0 & 21.2 & 24.9 & 23.0 & 964.46 & 992.05 & $1,169.64$ & $1,373.15$ & $1,269.33$ & $5,768.63$ & 55.14 \\
\hline 155B-1 & 18.0 & 18.7 & 22.0 & 22.9 & 23.0 & 896.41 & 933.78 & $1,097.45$ & $1,145.28$ & $1,146.21$ & $5,219.13$ & 49.92 \\
\hline $156 \mathrm{~A}-1$ & 18.0 & 18.1 & 34.4 & 47.6 & 47.3 & 258.99 & 260.10 & 495.95 & 685.85 & 681.77 & $2,382.66$ & 14.40 \\
\hline $157 A-1$ & 18.0 & 16.9 & 20.1 & 19.9 & 24.0 & 674.80 & 636.93 & 755.89 & 749.37 & 900.30 & $3,717.29$ & 37.58 \\
\hline $157 C-1$ & 18.0 & 17.0 & 21.1 & 20.1 & 25.0 & 96.61 & 91.47 & 113.15 & 107.90 & 134.07 & 543.20 & 5.37 \\
\hline 157D-1 & 18.0 & 17.0 & 21.6 & 20.9 & 25.0 & $1,072.06$ & $1,012.51$ & $1,288.16$ & $1,247.14$ & $1,490.31$ & $6,110.18$ & 59.71 \\
\hline $158 \mathrm{~A}-1$ & 18.0 & 19.0 & 21.3 & 20.0 & 27.0 & 941.32 & 994.16 & $1,112.08$ & $1,044.16$ & 0.05 & $5,501.77$ & 52.23 \\
\hline 158B-1 & 18.0 & 19.0 & 19.0 & 22.7 & 23.0 & 924.85 & 977.00 & 974.33 & $1,165.25$ & $1,183.34$ & $5,224.77$ & 51.36 \\
\hline 159A-1 & 22.1 & 22.9 & 29.1 & 37.4 & 35.0 & 68.55 & 71.08 & 90.14 & 116.01 & 108.61 & 454.39 & 3.10 \\
\hline $160 \mathrm{~A}-1$ & 17.5 & 21.1 & 19.8 & 20.0 & 26.1 & 924.07 & $1,115.94$ & $1,050.71$ & $1,057.87$ & $1,380.71$ & $5,529.30$ & 52.94 \\
\hline $160 \mathrm{~B}-1$ & 18.0 & 21.0 & 20.9 & 23.0 & 25.6 & $1,080.05$ & $1,259.85$ & $1,252.55$ & $1,380.11$ & $1,537.65$ & $6,510.21$ & 60.06 \\
\hline $161 \mathrm{~A}-1$ & 18.0 & 20.9 & 30.6 & 42.7 & 41.5 & 230.21 & 268.17 & 392.91 & 547.87 & 532.53 & $1,971.69$ & 12.82 \\
\hline $162 \mathrm{~A}-1$ & 20.1 & 17.9 & 22.8 & 24.0 & 26.2 & $1,244.31$ & $1,105.06$ & $1,410.78$ & $1,486.12$ & $1,621.56$ & $6,867.83$ & 61.90 \\
\hline 162B-1 & 20.0 & 14.5 & 19.2 & 23.9 & 23.0 & 769.04 & 560.05 & 738.98 & 918.84 & 884.90 & $3,871.81$ & 38.51 \\
\hline $163 \mathrm{~A}-1$ & 21.0 & 20.5 & 28.3 & 40.8 & 31.8 & 62.84 & 61.22 & 84.68 & 121.93 & 95.13 & 425.80 & 2.99 \\
\hline $164 A-1$ & 20.0 & 16.0 & 19.4 & 22.4 & 25.7 & $1,099.06$ & 879.28 & $1,067.84$ & $1,230.00$ & $1,410.51$ & $5,686.69$ & 54.91 \\
\hline $164 \mathrm{~B}-1$ & 19.5 & 16.4 & 19.7 & 23.1 & 25.1 & $1,246.27$ & $1,051.72$ & $1,261.58$ & $1,477.80$ & $1,607.82$ & $6,645.19$ & 64.06 \\
\hline $165 A-1$ & 18.9 & 19.2 & 30.4 & 43.6 & 32.8 & 252.25 & 256.95 & 405.95 & 582.97 & 438.65 & $1,936.77$ & 13.37 \\
\hline $166 \mathrm{~A}-1 / 2$ & 19.9 & 16.9 & 21.9 & 25.8 & 25.6 & $1,243.62$ & $1,055.00$ & $1,370.18$ & $1,609.59$ & $1,603.05$ & $6,881.44$ & 62.50 \\
\hline
\end{tabular}


R. R. Little January 15,2020 RE-01-20

Page 3 of 8

Table 1. Summary of ATR Power History by Cycle

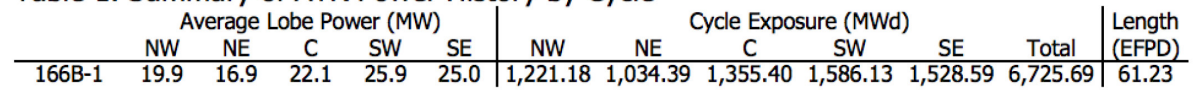

Table 2. Cumulative Exposure

\begin{tabular}{|c|c|c|c|c|c|c|}
\hline & \multicolumn{6}{|c|}{ Cumulative Exposure (MWd) } \\
\hline & NW & $\mathrm{NE}$ & C & SW & SE & Total \\
\hline $134 A-1$ & - & 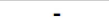 & 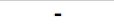 & - & - & - \\
\hline $134 A-2$ & 0.21 & 0.30 & 0.39 & 0.36 & 0.36 & 1.62 \\
\hline 134B-1 & 453.03 & 452.54 & 615.86 & 578.84 & 628.94 & $2,729.21$ \\
\hline 134B-2 & 838.65 & 838.34 & $1,169.36$ & $1,072.08$ & $1,165.01$ & $5,083.44$ \\
\hline $135 A-1$ & $1,074.81$ & $1,074.33$ & $1,560.43$ & $1,440.71$ & $1,628.99$ & $6,779.27$ \\
\hline 135B-1 & $1,533.09$ & $1,532.68$ & $2,191.22$ & $2,025.98$ & $2,265.50$ & $9,548.47$ \\
\hline 135B-2 & $1,938.11$ & $1,938.38$ & $2,758.60$ & $2,543.80$ & $2,828.49$ & $12,007.38$ \\
\hline $135 \mathrm{C}-1$ & $2,668.02$ & $2,668.08$ & $3,772.05$ & $3,476.80$ & $3,842.35$ & $16,427.30$ \\
\hline $136 \mathrm{~A}-1$ & $3,584.20$ & $3,584.47$ & 4,990.96 & $4,646.27$ & $5,012.52$ & $21,818.42$ \\
\hline 136B-1 & $4,286.14$ & $4,286.79$ & $5,922.15$ & $5,543.20$ & $5,909.49$ & $25,947.77$ \\
\hline 137A-1 & $5,261.49$ & $5,261.55$ & $7,258.31$ & $6,626.40$ & $7,262.80$ & $31,670.55$ \\
\hline 137B-1 & $5,503.46$ & $5,478.56$ & $7,687.93$ & $7,311.78$ & $7,630.43$ & $33,612.16$ \\
\hline 138A-1 & $6,550.33$ & 6,526.25 & $9,058.62$ & $8,648.17$ & $9,083.90$ & $39,867.27$ \\
\hline 138B-1 & 8.87 & 7,3 & $10,143.41$ & $9,719.11$ & $10,248.54$ & $44,865.80$ \\
\hline 139A-1 & & & & $10,905.3$ & $11,538.01$ & $50,430.43$ \\
\hline 139B-1 & 9 & & & 6 & & $55,807.19$ \\
\hline $140 \mathrm{~A}-1$ & 10, & & & 1 & & 33.33 \\
\hline $140 \mathrm{E}$ & 10 & & & & & 60 \\
\hline 141 & & & & & & 8.26 \\
\hline $142 A-1$ & 12, & 12, & & & 16,45 & $73,210.49$ \\
\hline 142B-1 & $13,601.10$ & $13,065.80$ & $17,618.34$ & $17,252.80$ & $17,750.62$ & $79,288.66$ \\
\hline $143 \mathrm{~A}-1 / 2$ & $14,481.08$ & $13,948.32$ & $18,806.01$ & $18,568.24$ & $18,973.70$ & $84,777.35$ \\
\hline 143B-1 & $15,513.14$ & $14,980.90$ & $20,229.54$ & $20,111.98$ & $20,408.71$ & $91,244.27$ \\
\hline $144 A-1$ & $16,300.11$ & $15,767.92$ & $21,236.21$ & $21,116.51$ & $21,502.15$ & $95,922.90$ \\
\hline 144B-1 & $17,232.36$ & $16,701.35$ & $22,391.95$ & $22,307.23$ & $22,693.04$ & $101,325.93$ \\
\hline $145 A-1$ & $18,215.33$ & $17,682.24$ & $23,659.29$ & $23,606.72$ & $24,100.79$ & $107,264.37$ \\
\hline 145B-1 & $19,235.79$ & $18,702.20$ & $24,980.72$ & $25,014.51$ & $25,579.04$ & $113,512.26$ \\
\hline $146 \mathrm{~A}-1$ & $20,142.55$ & $19,609.00$ & $26,206.46$ & $26,314.53$ & $26,891.59$ & $119,164.13$ \\
\hline 146B-1 & 21,0 & $20,316.11$ & 27,228 & $27,218.38$ & $27,912.55$ & $123,721.32$ \\
\hline 147A-1 & $22,203.09$ & $21,220.53$ & $28,436.46$ & $28,267.80$ & $29,067.70$ & $129,195.58$ \\
\hline $148 \mathrm{~A}-1$ & $23,059.06$ & $22,076.28$ & $29,557.89$ & $29,311.59$ & $30,161.34$ & $134,166.16$ \\
\hline 148B-1 & 23,9 & 23,0 & 49 & $30,535.57$ & $31,346.37$ & $139,610.99$ \\
\hline 149A-1 & 24,6 & 23,6 & 31, & 31, & 32, & $143,557.45$ \\
\hline 149B-1 & 25 & 24, & & 8 & & 5.46 \\
\hline $150 \mathrm{~A}-1$ & & & 33 & 19 & $33,856.53$ & $150,972.69$ \\
\hline & & & & & 34,822 & $155,505.69$ \\
\hline $151 A-1$ & $27,738.27$ & $26,406.37$ & & & $36,111.57$ & $161,214.71$ \\
\hline 151B-1/2 & $28,709.68$ & $27,148.12$ & $36,690.93$ & $36,584.38$ & 37,291 . & $166,424.65$ \\
\hline $152 A-1 / 6$ & 28 & 27,1 & & 36,584 & 37,292 & $166,426.65$ \\
\hline 152B-1 & $29,676.40$ & $27,961.42$ & $37,832.71$ & $37,756.86$ & $38,465.61$ & $171,693.00$ \\
\hline 153B-1 & $29,941.60$ & $28,226.77$ & $38,247.18$ & $38,232.98$ & $39,057.03$ & $173,705.56$ \\
\hline 154A-1 & & & & & $40,261.25$ & $178,937.30$ \\
\hline 154B-1 & $32,056.32$ & $30,023.84$ & $40,472.96$ & $40,524.89$ & $41,536.52$ & $184,614.53$ \\
\hline
\end{tabular}


R. R. Little January 15,2020

RE-01-20

Page 4 of 8

Table 2. Cumulative Exposure

\begin{tabular}{ccccccc} 
& \multicolumn{5}{c}{ Cumulative Exposure (MWd) } \\
& NW & NE & C & SW & SE & Total \\
\hline $155 A-1$ & $33,020.78$ & $31,015.89$ & $41,642.60$ & $41,898.04$ & $42,805.85$ & $190,383.16$ \\
$155 B-1$ & $33,917.19$ & $31,949.67$ & $42,740.05$ & $43,043.32$ & $43,952.06$ & $195,602.29$ \\
$156 \mathrm{~A}-1$ & $34,176.18$ & $32,209.77$ & $43,236.00$ & $43,729.17$ & $44,633.83$ & $197,984.95$ \\
$157 \mathrm{~A}-1$ & $34,850.98$ & $32,846.70$ & $43,991.89$ & $44,478.54$ & $45,534.13$ & $201,702.24$ \\
$157 \mathrm{C}-1$ & $34,947.59$ & $32,938.17$ & $44,105.04$ & $44,586.44$ & $45,668.20$ & $202,245.44$ \\
\hline $157 \mathrm{D}-1$ & $36,019.65$ & $33,950.68$ & $45,393.20$ & $45,833.58$ & $47,158.51$ & $208,355.62$ \\
$158 \mathrm{~A}-1$ & $36,960.97$ & $34,944.84$ & $46,505.28$ & $46,877.74$ & $48,568.56$ & $213,857.39$ \\
$158 \mathrm{~B}-1$ & $37,885.82$ & $35,921.84$ & $47,479.61$ & $48,042.99$ & $49,751.90$ & $219,082.16$ \\
$159 \mathrm{~A}-1$ & $37,954.37$ & $35,992.92$ & $47,569.75$ & $48,159.00$ & $49,860.51$ & $219,536.55$ \\
$160 \mathrm{~A}-1$ & $38,878.44$ & $37,108.86$ & $48,620.46$ & $49,216.87$ & $51,241.22$ & $225,065.85$ \\
$160 \mathrm{~B}-1$ & $39,958.49$ & $38,368.71$ & $49,873.01$ & $50,596.98$ & $52,778.87$ & $231,576.06$ \\
\hline $161 \mathrm{~A}-1$ & $40,188.70$ & $38,636.88$ & $50,265.92$ & $51,144.85$ & $53,311.40$ & $233,547.75$ \\
$162 \mathrm{~A}-1$ & $41,433.01$ & $39,741.94$ & $51,676.70$ & $52,630.97$ & $54,932.96$ & $240,415.58$ \\
$162 \mathrm{~B}-1$ & $42,202.05$ & $40,301.99$ & $52,415.68$ & $53,549.81$ & $55,817.86$ & $244,287.39$ \\
$163 \mathrm{~A}-1$ & $42,264.89$ & $40,363.21$ & $52,500.36$ & $53,671.74$ & $55,912.99$ & $244,713.19$ \\
$164 \mathrm{~A}-1$ & $43,363.95$ & $41,242.49$ & $53,568.20$ & $54,901.74$ & $57,323.5$ & $250,399.88$ \\
$164 \mathrm{~B}-1$ & $44,610.22$ & $42,294.21$ & $54,829.78$ & $56,379.54$ & $58,931.32$ & $257,045.07$ \\
$165 \mathrm{~A}-1$ & $44,862.47$ & $42,551.16$ & $55,235.73$ & $56,962.51$ & $59,369.97$ & $258,981.84$ \\
$166 \mathrm{~A}-1 / 2$ & $46,106.09$ & $43,606.16$ & $56,605.91$ & $58,572.10$ & $60,973.02$ & $265,863.28$ \\
$166 \mathrm{~B}-1$ & $47,327.27$ & $44,640.55$ & $57,961.31$ & $60,158.23$ & $62,501.61$ & $272,588.97$
\end{tabular}

Table 3. Startup and Shutdown Dates

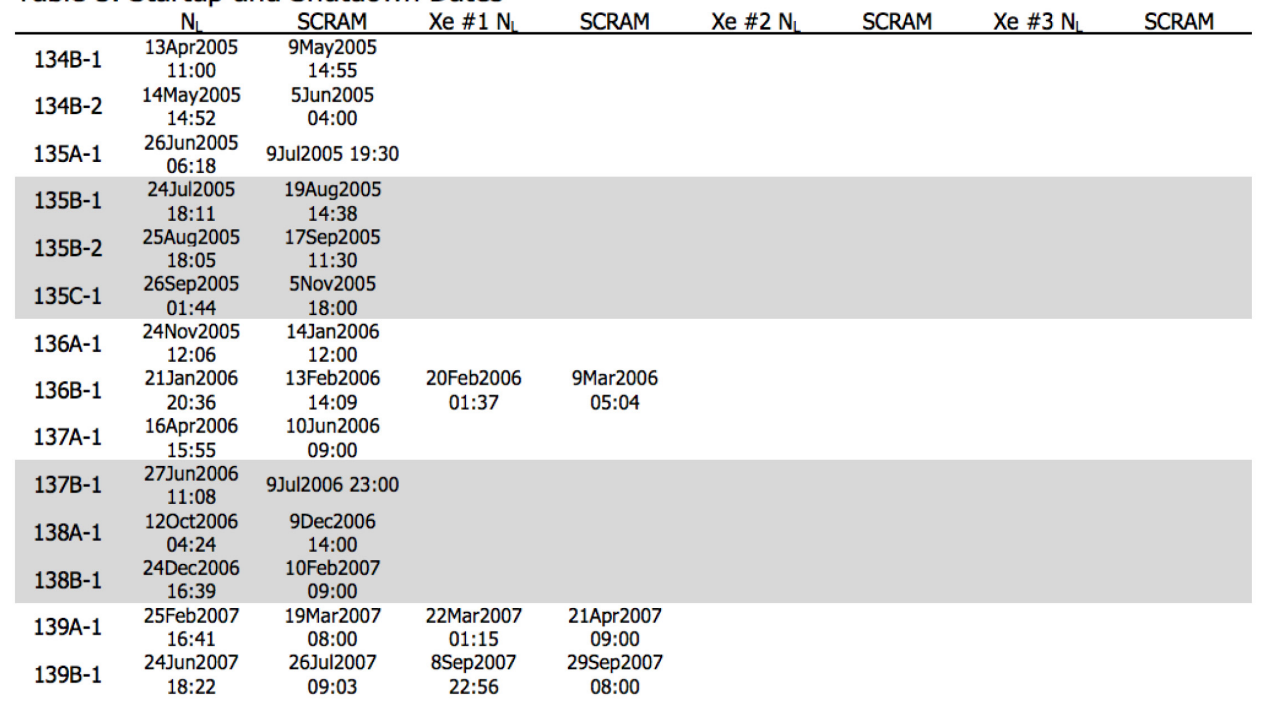


R. R. Little

January 15,2020

RE-01-20

Page 5 of 8

Table 3. Startup and Shutdown Dates

\begin{tabular}{|c|c|c|c|c|c|c|c|c|}
\hline & $\mathrm{N}_{\mathrm{L}}$ & SCRAM & $\mathrm{Xe} \# 1 \mathrm{~N}_{\mathrm{L}}$ & SCRAM & $\mathrm{Xe} \# 2 \mathrm{~N}_{\mathrm{L}}$ & SCRAM & $\mathrm{Xe} \# 3 \mathrm{~N}_{\mathrm{L}}$ & SCRAM \\
\hline $140 A-1$ & $\begin{array}{c}150 c t 2007 \\
14: 43\end{array}$ & $\begin{array}{c}\text { 1Dec2007 } \\
08: 00\end{array}$ & & & & & & \\
\hline $140 \mathrm{~B}-1$ & $\begin{array}{c}\text { 15Dec2007 } \\
15: 55\end{array}$ & $\begin{array}{c}\text { 9Jan2008 } \\
18: 20\end{array}$ & $\begin{array}{c}\text { 15Jan2008 } \\
02: 08\end{array}$ & $\begin{array}{c}\text { 26Jan2008 } \\
09: 59\end{array}$ & & & & \\
\hline $141 \mathrm{~A}-1$ & $\begin{array}{c}\text { 4Feb2008 } \\
19: 45\end{array}$ & $\begin{array}{c}8 \mathrm{Mar} 2008 \\
10: 00\end{array}$ & & & & & & \\
\hline $142 A-1$ & $\begin{array}{c}30 \text { Apr2008 } \\
15: 25\end{array}$ & $\begin{array}{c}1 \text { May2008 } \\
08: 48\end{array}$ & $\begin{array}{c}2 \text { May2008 } \\
02: 48\end{array}$ & $\begin{array}{c}\text { 3Jun2008 } \\
06: 57\end{array}$ & $\begin{array}{c}\text { 5Jun2008 } \\
\text { 09:26 }\end{array}$ & $\begin{array}{c}\text { 21Jun2008 } \\
11: 00\end{array}$ & & \\
\hline 142B-1 & 4Jul2008 13:31 & 6Jul2008 00:30 & 8Jul2008 19:18 & $\begin{array}{c}\text { 5Aug2008 } \\
15: 00\end{array}$ & $\begin{array}{c}\text { 7Aug2008 } \\
13: 02\end{array}$ & $\begin{array}{l}\text { 30Aug2008 } \\
21: 00\end{array}$ & & \\
\hline $143 A-1$ & $\begin{array}{l}\text { 23Sep2008 } \\
13: 19\end{array}$ & $\begin{array}{c}160 \mathrm{ct} 2008 \\
12: 35\end{array}$ & $\begin{array}{c}\text { 5Nov2008 } \\
06: 44\end{array}$ & $\begin{array}{c}\text { 21Nov2008 } \\
10: 05\end{array}$ & & & & \\
\hline $143 A-2$ & $\begin{array}{c}26 \text { Nov2008 } \\
03: 32\end{array}$ & $\begin{array}{c}\text { 6Dec2008 } \\
\text { 09:06 }\end{array}$ & & & & & & \\
\hline 143B-1 & $\begin{array}{c}\text { 22Dec2008 } \\
13: 41\end{array}$ & $\begin{array}{c}\text { 19Jan2009 } \\
09: 03\end{array}$ & $\begin{array}{c}\text { 21Jan2009 } \\
02: 06\end{array}$ & $\begin{array}{c}20 \mathrm{Feb} 2009 \\
10: 00\end{array}$ & & & & \\
\hline $144 A-1$ & $\begin{array}{c}12 \text { Mar2009 } \\
09: 01\end{array}$ & $\begin{array}{c}25 \mathrm{Apr} 2009 \\
09: 54\end{array}$ & & & & & & \\
\hline 144B-1 & $\begin{array}{c}\text { 10May2009 } \\
15: 51\end{array}$ & $\begin{array}{c}\text { 31May2009 } \\
18: 31\end{array}$ & $\begin{array}{l}\text { 3Jun2009 } \\
13: 00\end{array}$ & 4Jul2009 10:06 & & & & \\
\hline $145 A-1$ & $\begin{array}{c}\text { 5Sep2009 } \\
03: 02\end{array}$ & $\begin{array}{l}\text { 29Sep2009 } \\
\text { 08:32 }\end{array}$ & $\begin{array}{c}10 c t 2009 \\
02: 29\end{array}$ & $\begin{array}{c}80 c t 2009 \\
01: 48\end{array}$ & $\begin{array}{c}100 c t 2009 \\
13: 08\end{array}$ & $\begin{array}{c}120 c t 2009 \\
18: 09\end{array}$ & $\begin{array}{c}150 c t 2009 \\
04: 23\end{array}$ & $\begin{array}{c}\text { 6Nov2009 } \\
05: 17\end{array}$ \\
\hline 145B-1 & $\begin{array}{c}22 \text { Nov2009 } \\
12: 16\end{array}$ & $\begin{array}{c}\text { 1Dec2009 } \\
12: 01\end{array}$ & $\begin{array}{c}\text { 4Dec2009 } \\
08: 06\end{array}$ & $\begin{array}{c}\text { 23Jan2010 } \\
05: 00\end{array}$ & & & & \\
\hline $146 \mathrm{~A}-1$ & $\begin{array}{l}\text { 7Feb2010 } \\
20: 55\end{array}$ & $\begin{array}{c}14 \mathrm{Feb} 2010 \\
13: 51\end{array}$ & $\begin{array}{c}\text { 17Feb2010 } \\
22: 26\end{array}$ & $\begin{array}{c}3 \text { Apr2010 } \\
10: 00\end{array}$ & & & & \\
\hline 146B-1 & $\begin{array}{c}20 \text { Apr } 2010 \\
20: 54\end{array}$ & $\begin{array}{c}\text { 30May2010 } \\
08: 36\end{array}$ & & & & & & \\
\hline $147 A-1$ & $\begin{array}{c}\text { 22Jun2010 } \\
15: 35\end{array}$ & $\begin{array}{l}\text { 23Jul2010 } \\
05: 11\end{array}$ & $\begin{array}{c}\text { 25Jul2010 } \\
07: 37\end{array}$ & $\begin{array}{c}14 \text { Aug2 } 2010 \\
10: 05\end{array}$ & & & & \\
\hline $148 \mathrm{~A}-1$ & $\begin{array}{c}31 \text { Aug2010 } \\
18: 53\end{array}$ & $\begin{array}{c}120 c t 2010 \\
20: 35\end{array}$ & $\begin{array}{c}170 c t 2010 \\
14: 32\end{array}$ & $\begin{array}{c}230 \mathrm{ct} 2010 \\
10: 00\end{array}$ & & & & \\
\hline 148B-1 & $\begin{array}{c}17 \text { Nov } 2010 \\
14: 46\end{array}$ & $\begin{array}{c}\text { 8Jan2011 } \\
10: 00\end{array}$ & & & & & & \\
\hline $149 A-1$ & $\begin{array}{c}\text { 14Apr2011 } \\
01: 27\end{array}$ & $\begin{array}{c}21 \text { May2011 } \\
10: 00\end{array}$ & & & & & & \\
\hline 149B-1 & $\begin{array}{l}\text { 6Jun2011 } \\
11: 18\end{array}$ & $\begin{array}{l}\text { 30Jul2011 } \\
10: 00\end{array}$ & & & & & & \\
\hline $\begin{array}{l}150 \mathrm{~A}-1 \\
150 \mathrm{~B}-1\end{array}$ & $\begin{array}{c}\text { 19Aug2011 } \\
11: 42 \\
150 c t 2011 \\
00: 19\end{array}$ & $\begin{array}{c}\text { 1Sep2011 } \\
03: 43 \\
26 \text { Nov2011 } \\
10: 00\end{array}$ & & & & & & \\
\hline $151 \mathrm{~A}-1$ & $\begin{array}{l}\text { 14Dec2011 } \\
\text { 00:14 }\end{array}$ & $\begin{array}{l}\text { 25Dec2011 } \\
09: 17\end{array}$ & $\begin{array}{l}\text { 28Dec2011 } \\
\text { 03:50 }\end{array}$ & $\begin{array}{c}11 \mathrm{Feb} 2012 \\
10: 00\end{array}$ & & & & \\
\hline 151B-1 & $\begin{array}{c}1 \text { Mar2012 } \\
05: 14\end{array}$ & $\begin{array}{c}\text { 22Mar2012 } \\
08: 07\end{array}$ & $\begin{array}{c}25 \mathrm{Mar} 2012 \\
08: 18\end{array}$ & $\begin{array}{c}27 \text { Mar2012 } \\
14: 24\end{array}$ & & & & \\
\hline 151B-2 & $\begin{array}{c}\text { 6Apr2012 } \\
23: 09\end{array}$ & $\begin{array}{c}5 \text { May2012 } \\
10: 03\end{array}$ & & & & & & \\
\hline $152 A-1 / 6$ & $\begin{array}{c}\text { 15Aug2012 } \\
0800\end{array}$ & $\begin{array}{c}200 c t 2012 \\
0459\end{array}$ & & & & & & \\
\hline 152B-1 & $\begin{array}{c}27 \text { Nov2012 } \\
03: 39\end{array}$ & $\begin{array}{c}\text { 18Jan2013 } \\
10: 00\end{array}$ & & & & & & \\
\hline 153B-1 & $\begin{array}{c}29 \operatorname{Mar} 2013 \\
12: 34\end{array}$ & $\begin{array}{c}12 \mathrm{Apr} 2013 \\
18: 41\end{array}$ & & & & & & \\
\hline $154 A-1$ & $\begin{array}{c}\text { 19May2013 } \\
02: 36\end{array}$ & $\begin{array}{c}21 \text { May } 2013 \\
05: 13\end{array}$ & $\begin{array}{c}\text { 23May2013 } \\
19: 11\end{array}$ & $\begin{array}{l}\text { 13Jul2013 } \\
10: 00\end{array}$ & & & & \\
\hline
\end{tabular}


R. R. Little

January 15,2020

RE-01-20

Page 6 of 8

Table 3. Startup and Shutdown Dates

\begin{tabular}{|c|c|c|c|c|c|c|c|c|}
\hline & $\mathrm{N}_{\mathrm{L}}$ & SCRAM & $\mathrm{Xe} \# 1 \mathrm{~N}_{\mathrm{L}}$ & SCRAM & $\mathrm{Xe} \# 2 \mathrm{~N}_{\mathrm{L}}$ & SCRAM & $\mathrm{Xe} \# 3 \mathrm{~N}_{\mathrm{L}}$ & SCRAM \\
\hline 154B-1 & $\begin{array}{c}\text { 23Aug2013 } \\
14: 12\end{array}$ & $\begin{array}{c}160 c t 2013 \\
10: 03\end{array}$ & & & & & & \\
\hline $155 A-1$ & $\begin{array}{c}8 \text { Nov2013 } \\
00: 28\end{array}$ & $\begin{array}{c}\text { 13Nov2013 } \\
01: 37\end{array}$ & $\begin{array}{c}28 \text { Nov2013 } \\
02: 10\end{array}$ & $\begin{array}{c}\text { 17Jan2014 } \\
15: 05\end{array}$ & & & & \\
\hline 155B-1 & $\begin{array}{c}13 \mathrm{Feb} 2014 \\
04: 11\end{array}$ & $\begin{array}{c}\text { 21Mar2014 } \\
07: 19\end{array}$ & $\begin{array}{c}28 \operatorname{Mar} 2014 \\
16: 41\end{array}$ & $\begin{array}{c}\text { 12Apr2014 } \\
04: 00\end{array}$ & & & & \\
\hline $156 \mathrm{~A}-1$ & 29May2014 & 4Jun2014 & 7Jun2014 & 7Jun2014 & 10Jun2014 & 18Jun2014 & & \\
\hline $157 A-1$ & $\begin{array}{c}04: 26 \\
\text { 23Jul2014 } \\
05: 02\end{array}$ & $\begin{array}{c}16: 53 \\
\text { 30Aug2014 } \\
02: 42\end{array}$ & 01:02 & 21:35 & $13: 43$ & 21:00 & & \\
\hline 157C-1 & $\begin{array}{c}9 \text { Feb2015 } \\
17: 21\end{array}$ & $\begin{array}{c}15 F e b 2015 \\
08: 39\end{array}$ & & & & & & \\
\hline 157D-1 & $\begin{array}{c}\text { 29May2015 } \\
22: 00\end{array}$ & $\begin{array}{c}\text { 23Jul2015 } \\
\text { 18:01 }\end{array}$ & $\begin{array}{c}\text { 6Aug2015 } \\
21: 48\end{array}$ & $\begin{array}{c}\text { 12Aug2015 } \\
04: 00\end{array}$ & & & & \\
\hline $158 \mathrm{~A}-1$ & $\begin{array}{c}\text { 10Nov2015 } \\
03: 21\end{array}$ & $\begin{array}{c}\text { 2Jan2016 } \\
04: 06\end{array}$ & & & & & & \\
\hline 158B-1 & $\begin{array}{c}\text { 9Feb2016 } \\
16: 30\end{array}$ & $\begin{array}{c}\text { 1Apr2016 } \\
09: 00\end{array}$ & & & & & & \\
\hline 159A-1 & $\begin{array}{l}\text { 16Jun2016 } \\
22: 58\end{array}$ & $\begin{array}{c}\text { 23Jun2016 } \\
10: 32\end{array}$ & $\begin{array}{l}\text { 27Jun2016 } \\
\text { 02:09 }\end{array}$ & $\begin{array}{l}\text { 28Jun2016 } \\
18: 16\end{array}$ & & & & \\
\hline $160 \mathrm{~A}-1$ & $\begin{array}{c}\text { 16Sep2016 } \\
02: 34\end{array}$ & $\begin{array}{c}8 \text { Nov2016 } \\
11: 00\end{array}$ & & & & & & \\
\hline 160B-1 & $\begin{array}{l}\text { 19Dec2016 } \\
22: 07\end{array}$ & $\begin{array}{c}\text { 18Jan2017 } \\
16: 52\end{array}$ & $\begin{array}{c}\text { 23Jan2017 } \\
19: 08\end{array}$ & $\begin{array}{c}23 F e b 2017 \\
14: 33\end{array}$ & & & & \\
\hline $161 \mathrm{~A}-1$ & $\begin{array}{c}\text { 29May2017 } \\
03: 45\end{array}$ & $\begin{array}{c}\text { 11Jun2017 } \\
15: 41\end{array}$ & & & & & & \\
\hline $162 A-1$ & $\begin{array}{c}60 c t 2017 \\
05: 03\end{array}$ & $\begin{array}{l}\text { 7Dec2017 } \\
10: 59\end{array}$ & & & & & & \\
\hline $162 \mathrm{~B}-1$ & $\begin{array}{c}\text { 16Feb2018 } \\
01: 19\end{array}$ & $\begin{array}{c}9 \text { Mar2018 } \\
17: 35\end{array}$ & $\begin{array}{c}11 \text { Mar2018 } \\
22: 12\end{array}$ & $\begin{array}{c}29 \operatorname{Mar} 2018 \\
08: 06\end{array}$ & & & & \\
\hline $163 A-1$ & $\begin{array}{c}29 \mathrm{Apr} 2018 \\
11: 30\end{array}$ & $\begin{array}{c}\text { 8May2018 } \\
03: 38\end{array}$ & & & & & & \\
\hline $164 A-1$ & $\begin{array}{l}\text { 10Jun2018 } \\
18: 12\end{array}$ & $\begin{array}{l}\text { 24Jun2018 } \\
06: 46\end{array}$ & 3Jul2018 06:22 & $\begin{array}{l}\text { 30Jul2018 } \\
13: 13\end{array}$ & $\begin{array}{l}\text { 2Aug2018 } \\
08: 11\end{array}$ & $\begin{array}{c}\text { 17Aug2018 } \\
15: 06\end{array}$ & & \\
\hline 164B-1 & $\begin{array}{c}\text { 18Sep2018 } \\
21: 31\end{array}$ & $\begin{array}{c}150 c t 2018 \\
22: 06\end{array}$ & $\begin{array}{c}240 c t 2018 \\
03: 10\end{array}$ & $\begin{array}{l}\text { 4Nov2018 } \\
20: 06\end{array}$ & $\begin{array}{l}\text { 22Dec2018 } \\
\text { 05:36 }\end{array}$ & $\begin{array}{c}\text { 17Jan2019 } \\
15: 18\end{array}$ & & \\
\hline $165 \mathrm{~A}-1$ & $\begin{array}{c}28 \mathrm{Feb} 2019 \\
02: 47\end{array}$ & $\begin{array}{c}3 \text { Mar2019 } \\
10: 53\end{array}$ & 7Jun2019 20:54 & & & & & \\
\hline $166 \mathrm{~A}-1 / 2$ & $\begin{array}{l}\text { 25Jul2019 } \\
01: 03\end{array}$ & $\begin{array}{c}\text { 6Sep2019 } \\
09: 08\end{array}$ & $\begin{array}{c}\text { 16Sep2019 } \\
21: 28\end{array}$ & $60 c t 201910: 06$ & & & & \\
\hline 166B-1 & $\begin{array}{l}\text { 9Nov2019 } \\
16: 40\end{array}$ & $\begin{array}{c}10 \mathrm{Jan} 2020 \\
11: 30\end{array}$ & & & & & & \\
\hline
\end{tabular}


R. R. Little

January 15,2020

RE-01-20

Page 7 of 8

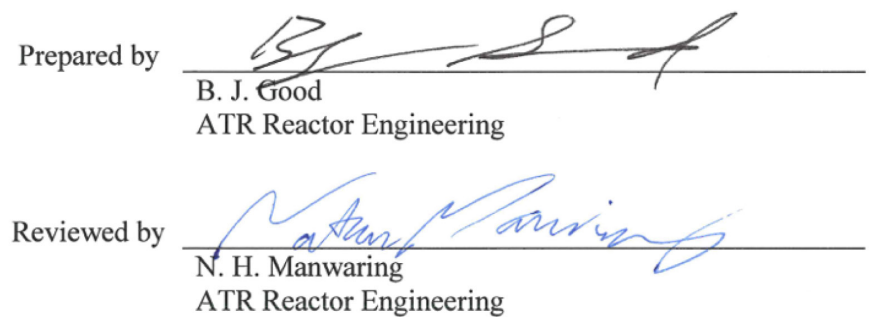


R. R. Little

January 15, 2020

RE-01-20

Page 8 of 8

cc:

D. S. Blight
J. O. Brower
H. D. Buckland
R. R. Bullock
K. H. Carlquist
C. D. Cooper
C. C. Dwight
K. R. Estes
S. Flores
B. J. Good
M. J. Guzman
M. R. Holtz
R. C. Howard
W. F. Jones
T. L. Julius
V. C. Kirkpatrick
M. A. Lillo
S. G. Louk
N. J. Lybeck
A. D. Maile
N. H. Manwaring

M. A. Marshall

C. A. McPhie, FMP

R. M. Murphy

D. R. Norman

D. Ogden

S. O'Kelly

R. B. Richardson

D. G. Robinson

D. L. Rowsell

D. J. Schoonen

K. A. Smith

L. D. Smith

K. D. Stueve

E. T. Swain

D. J. Utterbeck

K. L. Zimmer

ATR Document Management, email:

Jennifer.eversull@inl.gov

Cycle 166B-1 Surveillance File

Reactor Engineering Letter File (RE-01-20)

Uniform File Code: $\underline{8153}$

Disposition Authority: $\underline{\text { A17-32-b-1 }}$

Retention Schedule: Retain in accordance with current regulatory requirements or for nuclear facilities, 6 years after plant or item is put into operation.

NOTE: Original disposition authority, retention schedule, and Uniform Filing Code applied by the sender may not be appropriate for all recipients. Make adjustments as needed. 


\section{ATTACHMENT B}

Katie A. Anderson

Wed 1/29/2020 7:04 AM

Jason V. Brookman $\approx$

Hi Jason,

The NSUF program would like to request you to perform an as-run analysis of the 3 DPA capsules for the BSU-8242 experiment and document your analysis in the form of a tech checked ECAR.

Thanks,

Katie

Idaho National Laboratory

KATIE ANDERSON | Experiment Manager

Imadiation Testing

Idaho National Laboratory | 2525 North Fremont Ave. | Idaho Falls, ID 83415 | Mail Stop 3890

Phone: 208-526-0049 | Cell: 208-520-1197

Katie.anderson@inl.gov 


\section{ATTACHMENT C}

W $18 \mathrm{~KB}$

Hi Jason,

Due to the capsule misload at ATR, the program is requesting an analysis of the actual configuration with an update/revision to the ECAR as needed to reflect the true irradiation conditions. Please see the attached file which documents the discovery of the swap between capsules D and E and how they were found to have been irradiated in the wrong ATR positions. Please let me know if you have any questions.

\section{BSU-8242 Assembly/Capsule Pairing Issue \& Timeline}

Issue: On 27 May 2020, Advanced Test Reactor (ATR) Canal Operators discovered that two Boise State University experiment (BSU-8242) capsules were welded to the wrong assemblies. The assemblies have a long upper tube welded to the top of the capsule. Both the upper tube and the experiment capsule are marked with IDs. The ID on the upper tube is used for identification during reactor loading and canal handling. In preparation to ship the experiments, the upper tubes were cut off and the ID on the capsules was checked. At this point, it was discovered that nearly identical capsules from two BSU-8242 assemblies had been interchanged, resulting in a capsule swap between adjacent ATR experiment positions A7 and A8.

\begin{tabular}{|l|l|}
\hline Date (Time) & Event Description \\
\hline April 2018 & $\begin{array}{l}\text { Final assembly/welding of BSU-8242 occurred. 8242-D had a machining } \\
\text { issue with the "D" which resulted in the "D" being re-machined. }\end{array}$ \\
\hline $4 / 30 / 18$ & $\begin{array}{l}\text { Prior to welding, engineer G. Housley signs off that assembly components } \\
\text { looked correctly arranged, however, at that point the pieces were not } \\
\text { assembled (Step 180 in work package). Welding occurred after the engineer } \\
\text { left. }\end{array}$ \\
\hline $5 / 1 / 18$ & Green tag(s) is(are) issued for BSU-8242 assemblies. \\
\hline $5 / 20 / 20$ & $\begin{array}{l}\text { S. Evans requests confirmation of capsule and assembly IDs during cutting } \\
\text { to ensure the correct identifiers are used for shipping (top portion of the } \\
\text { experiment assembly is cut off prior to shipping) }\end{array}$ \\
\hline $5 / 27 / 20(\mathrm{pm})$ & $\begin{array}{l}\text { G. Schuldt, M. Hill and ATR Canal Ops discover markings do not match } \\
\text { between assembly and capsule IDs for BSU assemblies 8242-D and 8242- } \\
\text { E. They proceed with cutting based on assembly identifiers and use video }\end{array}$ \\
\hline
\end{tabular}




\begin{tabular}{|l|l|}
\hline & $\begin{array}{l}\text { recording to ensure capsule and assembly identifiers were captured prior to } \\
\text { cutting. }\end{array}$ \\
\hline $5 / 27 / 20(\sim 3 \mathrm{pm})$ & G. Schuldt calls S. Evans to report discrepancies. \\
\hline $\begin{array}{l}5 / 27 / 20(4: 30 \\
\text { pm) }\end{array}$ & $\begin{array}{l}\text { S. Evans sends email to K. Lombard, T. Skeen, R. Marlow, M. Hill, K. } \\
\text { Anderson, and G. Schuldt reporting discrepancy between drawings and } \\
\text { verbal report of as-found capsule and assembly pairing. }\end{array}$ \\
\hline $5 / 28 / 20($ am) & $\begin{array}{l}\text { Upon receipt of the cutting video, T. Skeen and S. Evans independently } \\
\text { confirm capsule and assembly IDs do not match drawings. }\end{array}$ \\
\hline $5 / 28 / 20$ (noon) & $\begin{array}{l}\text { S. Evans sends an email to K. Lombard and T. Skeen with photos from } \\
\text { video documenting swap of the capsules attached to assemblies 8242-D and } \\
8242-\text { E. Notification to ATR Operations management follows. }\end{array}$ \\
\hline $5 / 28 / 20$ & G. Schuldt (ATR Ops) notifies J. Duplessis (DOE)of the issue. \\
\hline
\end{tabular}

\section{Timeline:}

